\title{
Role of the host cell in the type III translocation of Pseudomonas aeruginosa exoenzyme $S$
}

\author{
Elizabeth A. Rucks \\ West Virginia University
}

Follow this and additional works at: https://researchrepository.wvu.edu/etd

\section{Recommended Citation}

Rucks, Elizabeth A., "Role of the host cell in the type III translocation of Pseudomonas aeruginosa exoenzyme S" (2005). Graduate Theses, Dissertations, and Problem Reports. 4188.

https://researchrepository.wvu.edu/etd/4188

This Dissertation is protected by copyright and/or related rights. It has been brought to you by the The Research Repository @ WVU with permission from the rights-holder(s). You are free to use this Dissertation in any way that is permitted by the copyright and related rights legislation that applies to your use. For other uses you must obtain permission from the rights-holder(s) directly, unless additional rights are indicated by a Creative Commons license in the record and/ or on the work itself. This Dissertation has been accepted for inclusion in WVU Graduate Theses, Dissertations, and Problem Reports collection by an authorized administrator of The Research Repository @ WVU.

For more information, please contact researchrepository@mail.wvu.edu. 
Role of the host cell in the type III translocation of Pseudomonas aeruginosa exoenzyme $S$

\title{
Elizabeth A. Rucks
}

Dissertation submitted to the School of Medicine at West Virginia University In partial fulfillment of the requirements for the degree of

\author{
Doctor of Philosophy \\ In \\ Microbiology and Immunology \\ Joan C. Olson (Chair) \\ Nyles W. Charon \\ Thomas A. Elliott \\ Edward L. Krug \\ Rosana Schafer \\ Cynthia F. Wright
}

Department of Microbiology, Immunology and Cell Biology

\author{
Morgantown, West Virginia \\ 2005
}

Keywords: Pseudomonas aeruginosa, exoenzyme S, type III secretion Copyright 2005 Elizabeth A. Rucks 


\begin{abstract}
Role of the host cell in the type III translocation of Pseudomonas aeruginosa exoenzyme $S$
\end{abstract}

Elizabeth A. Rucks

Exoenzyme $S$ (ExoS) is a bifunctional toxin that is directly translocated into eukaryotic cells by the Pseudomonas aeruginosa $(\mathrm{Pa}$ ) type III secretory (T3S) process. The Nterminal region of ExoS includes a GTPase activating protein (GAP) activity and the Cterminal region includes an ADP-ribosyltransferase (ADPRT) activity. The T3Stranslocation (T3S-T) of ExoS allows the bacteria to directly manipulate host cell function, thereby contributing the virulence of $\mathrm{Pa}$. A cell culture model of infection was developed to study the effects of ExoS on cell function, which differentiates the effects of ExoS expression from other bacterial effects. Previous studies found that ExoS inactivates cell function, and this inactivation was largely attributed to the ADPRT activity. Bacterially translocated ExoS (T3S-T-ExoS) was also found to ADP-ribosylate a number of low molecular weight G-proteins (LMWG-proteins) within the cell. While the effects of T3S-T-ExoS on cell function were characterized, the role of the host cell in the T3S process remained unknown. Studies described in this dissertation characterize host cell influences in the targeting of ExoS ADPRT activity to specific eukaryotic substrates, characterize host cell mechanism of resistance to ExoS, and examine mechanisms associated with differences in host cell influence on T3S-T-ExoS ADPRT activity. Results from these studies identified two main patterns of T3S-T ExoS ADPribosylation of eukaryotic proteins, a model system to examine the host cell influence on 
the Pa T3S-T process, and a method of tracking intracellular ExoS by examining ExoSeukaryotic protein interactions. Combined, these studies expand previous knowledge about the host cell influence on the $\mathrm{Pa}$ T3S-T process and provide methods to understand host cell mechanisms targeting T3S effector activity. 


\section{Dedication}

I would like to dedicate this work in memory of my two wonderful grandmothers, Elizabeth Vann (Nana) and Carol Verderaime (Grandmother V.).

To Nana, who understood how important my work is to me and was always encouraging me to stay true to myself and my goals.

To Grandmother V., who always wanted to hear about my experiments and for constantly grounding me in how research science is ultimately about improving the lives of many people versus accomplishing recognition for one person.

You both are greatly missed, but your influence and spirit will forever be recognized. 


\section{Acknowledgements}

To my mentor, Joan Olson: thank you for making sure that I got my money's worth (plus interest) out of grad school. The Olson lab is a great environment to learn and grow as a scientist.

To my Committee members from WVU, Nyles Charon, Thomas Elliott and Rosana Schafer: you always made me feel like I was your student from the beginning. To my Committee members from MUSC, Ed Krug and Cindy Wright: thank you for sticking with me through the move. All of your influences have prepared me well for the next step.

To my lab mates, Jenny Fraylick-Meredith, Claudia Rocha, John Shea, Gus DiNovo and Jen Salazar: you guys could always make lab a fun place to be, even when gels weren't working.

More thanks:

To Chris Cuff: for teaching me to "never let the challenge beat you".

To Dave Yelton: for being wise at all the right moments.

To Tim Vincent: for teaching me some of the experimental techniques that I used in my studies.

To Richard Bakker, Mark Blake, Cindy Cunningham, Sarah Dodson, Alicia Mathers, Jeff Miller and Melanie Sal: for your friendship, enthusiasm and long chain emails about food.

I would like to thank my family: without your love and support none of this would be possible:

Andy and Donna Rucks, Shannon Rucks, Nan Rucks, Vann Family, Verderaime Family

Last, but not least, I would like to extend a BIG THANK YOU to my wonderful fiancé, Dalen Moore. Thank you for being there for the great moments, the moments that just plain stunk, and all the moments in between. I love you to pieces! 


\section{Table of Contents}

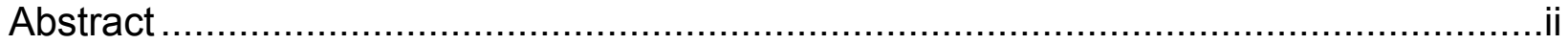

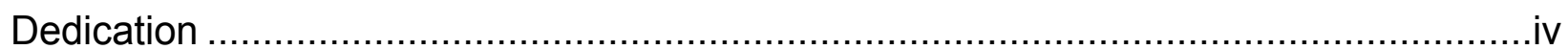

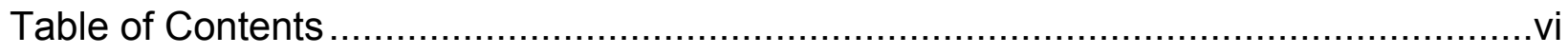

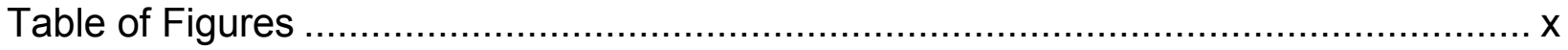

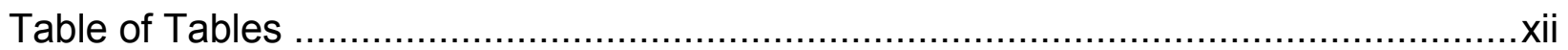

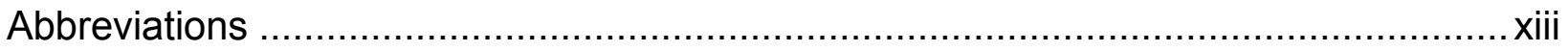

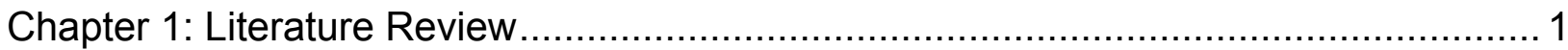

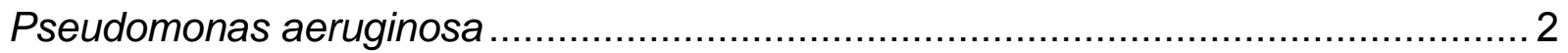

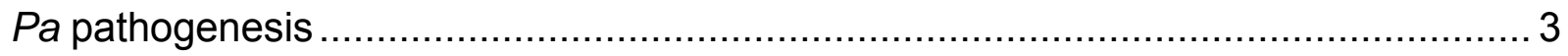

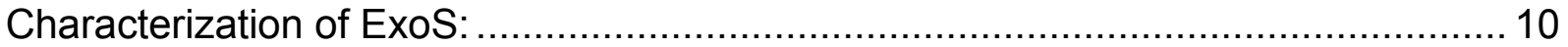

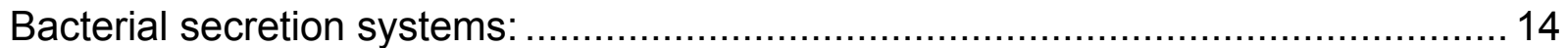

Characterization of $\mathrm{Pa}$ ExoS secretory mechanism: ............................................ 18

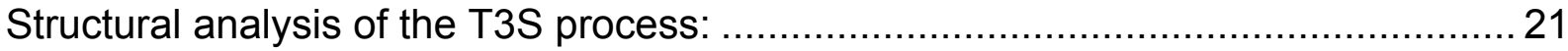

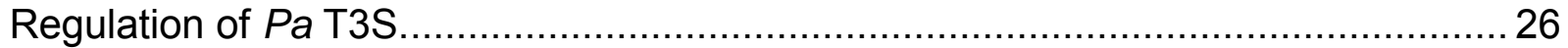

Manipulation of the host cell by T3S effector proteins: ....................................... 28

Structure/function relationships of T3S-T-ExoS. .................................................. 30

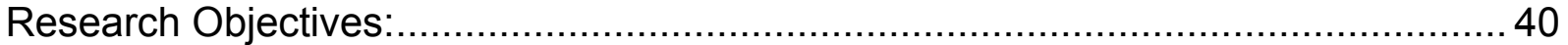

Chapter 2: Cell line differences in bacterially translocated ExoS ADP-ribosyltransferase

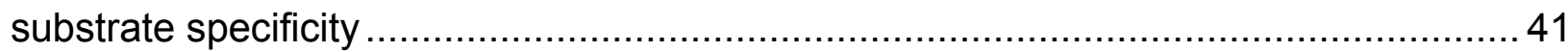

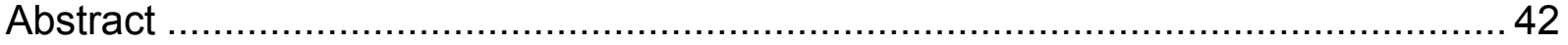

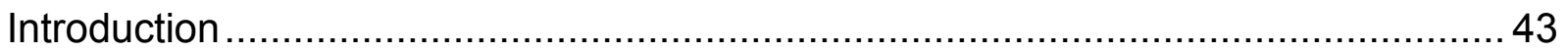

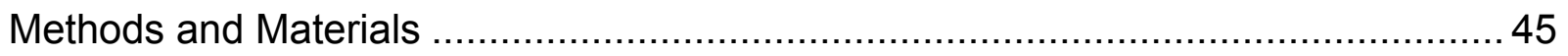

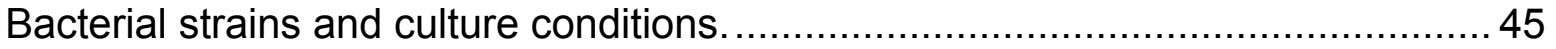

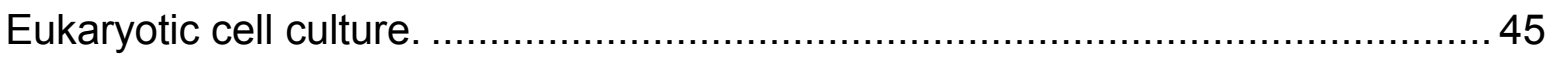

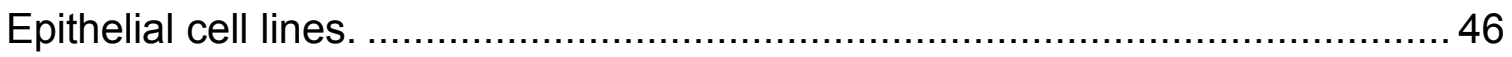

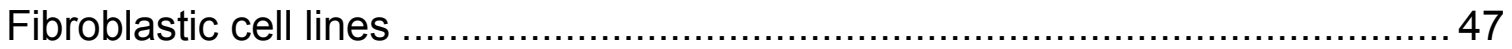

Co-culture of eukaryotic cells with bacteria................................................. 47

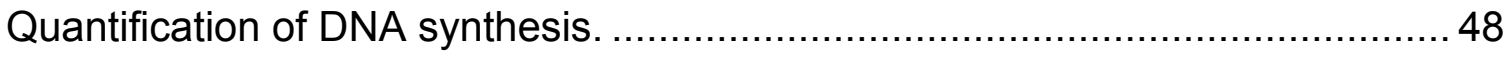

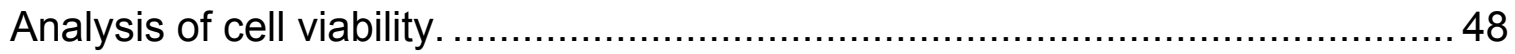

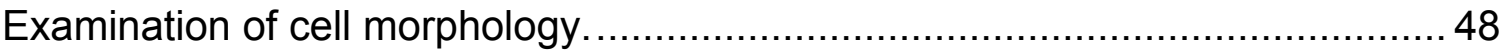




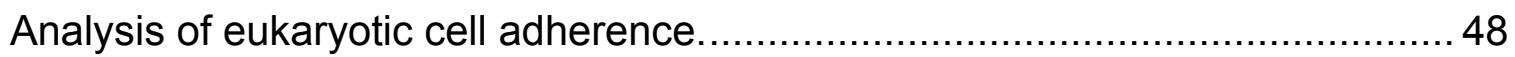

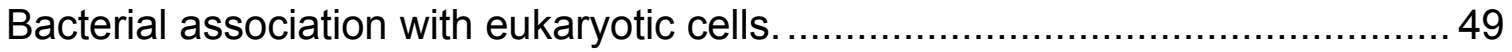

ADP-ribosylation of cellular substrates by bacterially translocated ExoS..............50

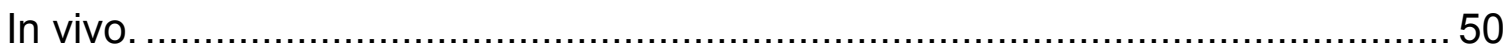

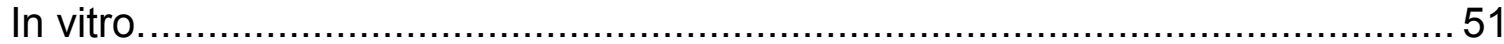

Time course analysis of ExoS internalization................................................ 51

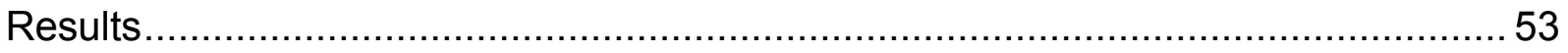

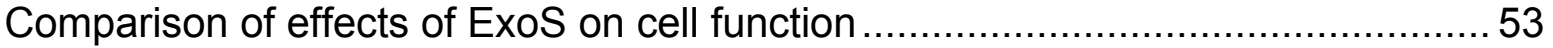

Comparison of bacterial association to cellular sensitivity to ExoS .......................58

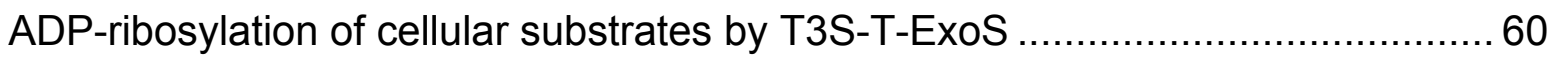

Time-course analysis of T3S-T and substrate modification by ExoS ADPRT activity.

Patterns of ExoS substrate modification in different cell lines. ............................. 66

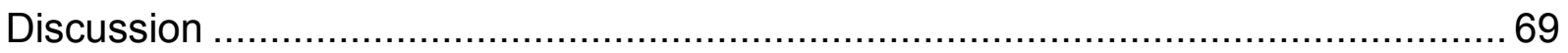

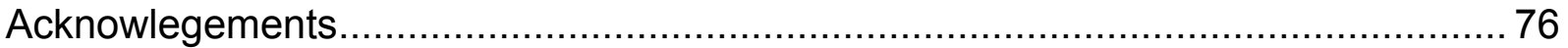

Chapter 3: Characterization of an ExoS type III translocation-resistant cell line ...........77

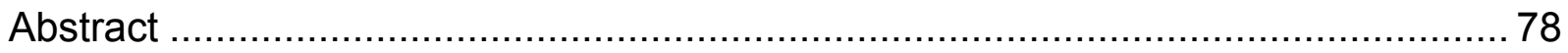

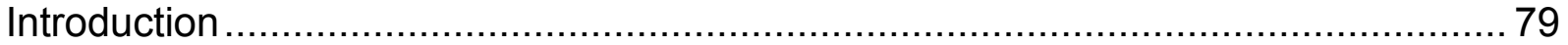

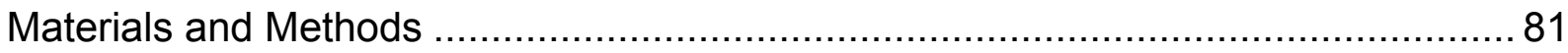

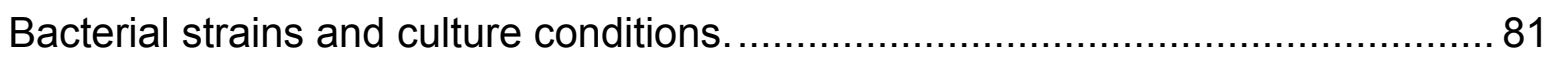

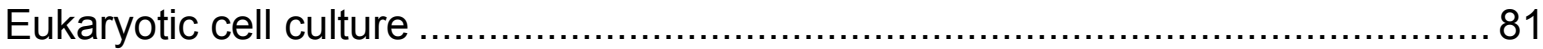

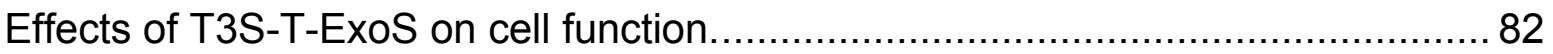

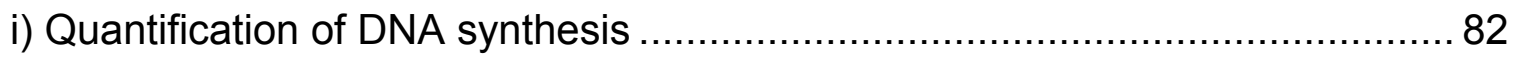

ii) Examination of cellular morphology by scanning electron microscopy (SEM) 82

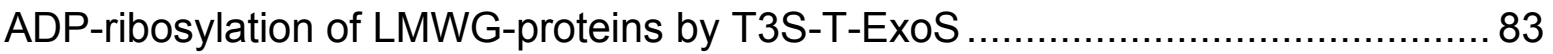

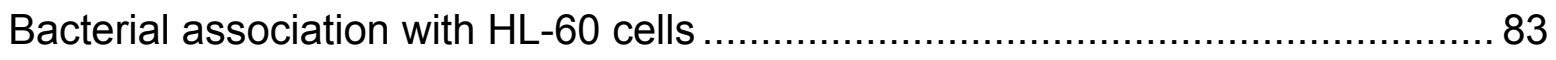

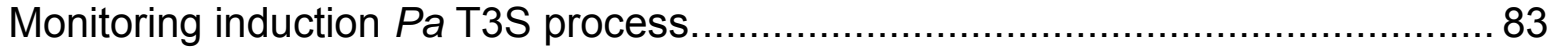

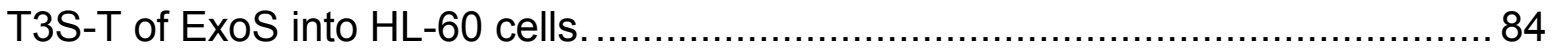

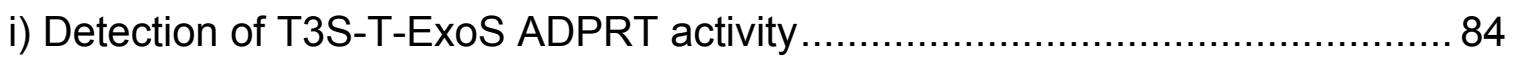

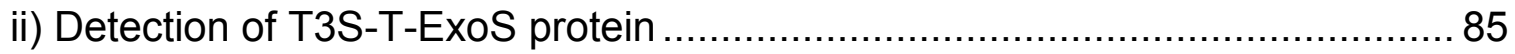

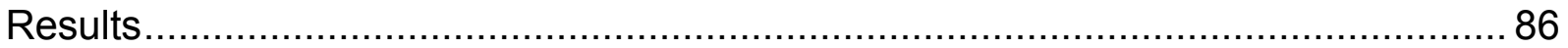




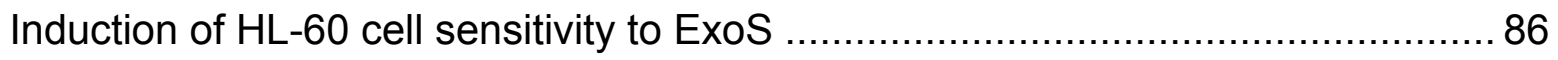

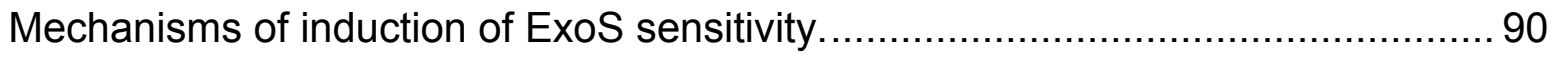

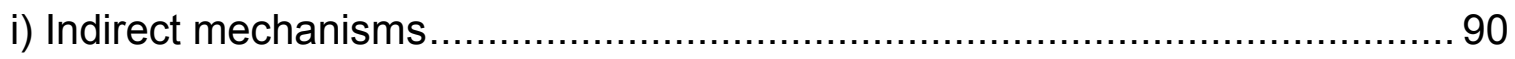

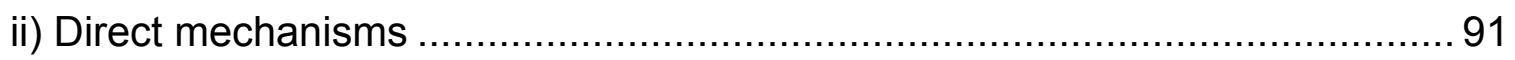

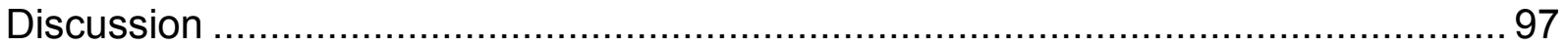

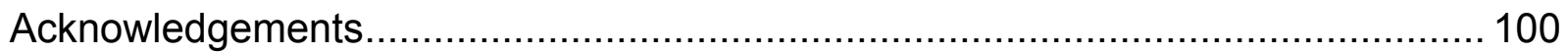

Chapter 4: Contribution of the host cell to the molecular targeting of type III translocated

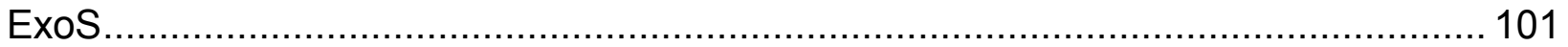

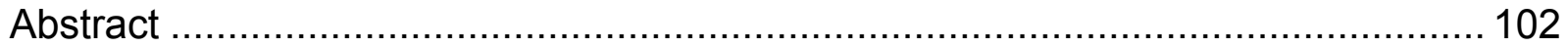

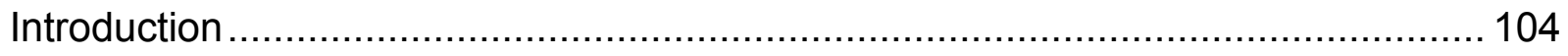

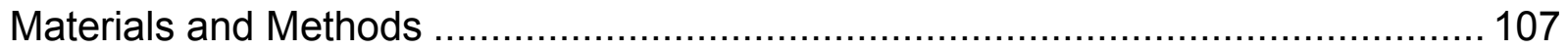

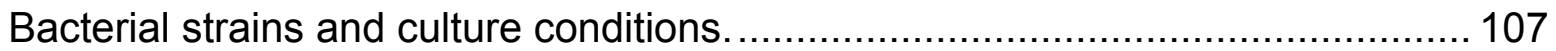

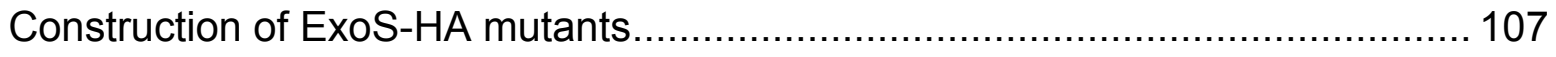

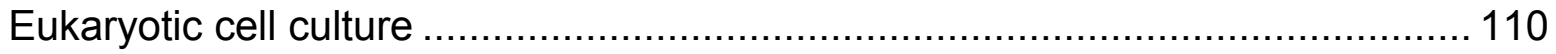

Monitoring ADP-ribosylation of eukaryotic substrates ..................................... 111

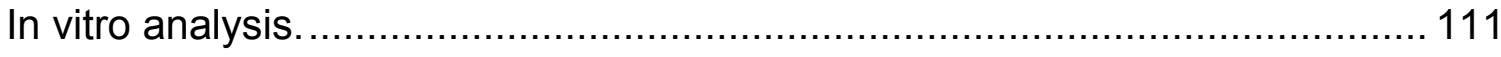

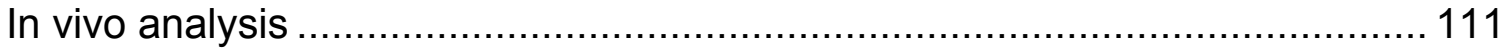

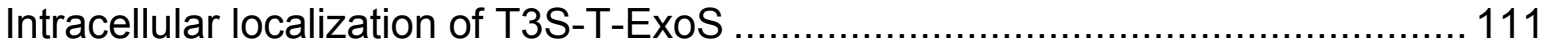

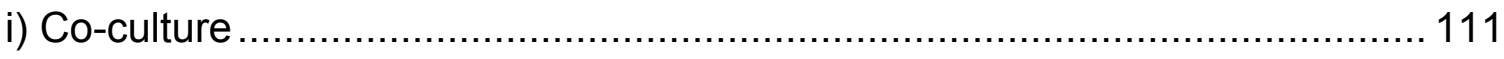

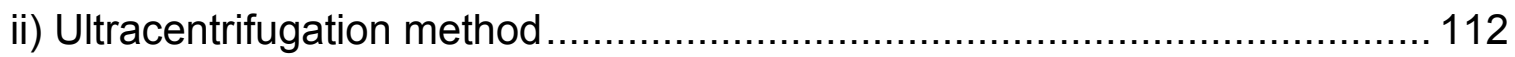

iii) Digitonin extraction ...................................................................... 113

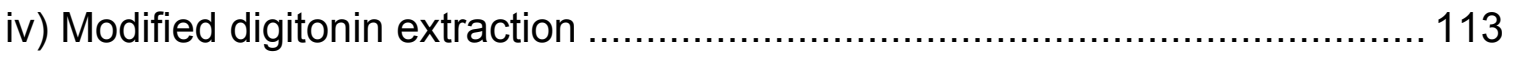

Examination of T3S-T-ExoS intracellular processing ..................................... 114

i) Effect of protease inhibitors on T3S-T-ExoS ADPRT activity........................ 114

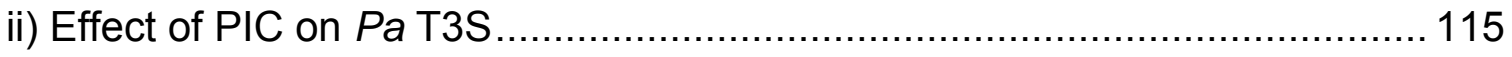

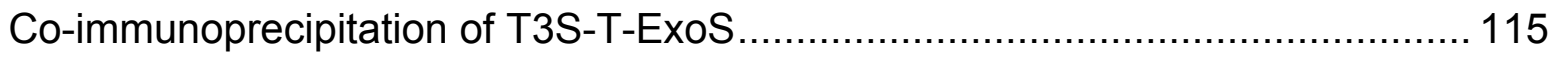

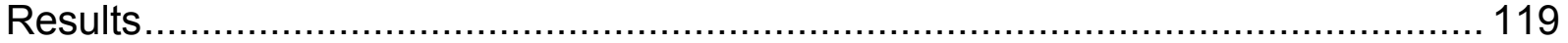

Effects of MLD, GAP and ADPRT mutations on T3S-T-ExoS eukaryotic substrate

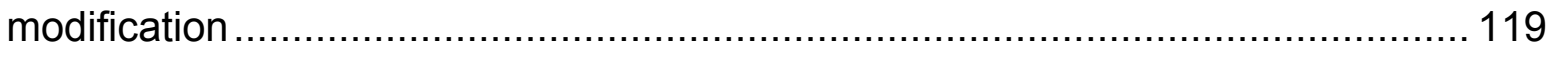

Examining intracellular processing of T3S-T-ExoS …................................... 128

Role of the R146 GAP residue in T3S-T of ExoS .......................................... 132 
T3S-T-ExoS protein interactions within rodent and human cell lines

Discussion

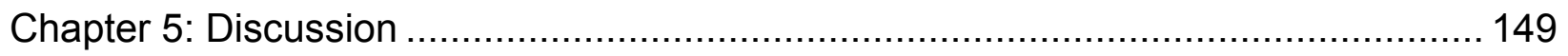

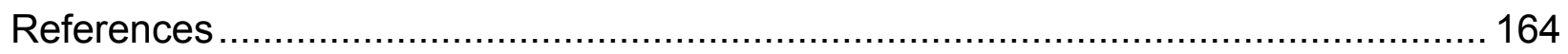

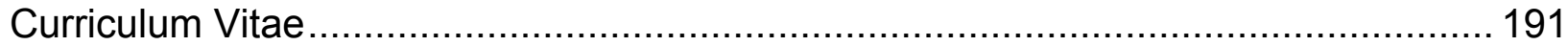

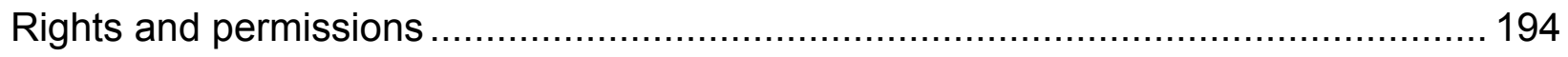




\section{Table of Figures}

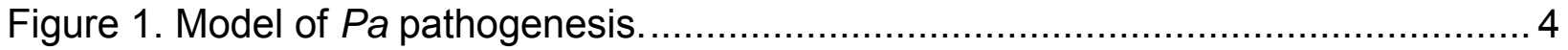

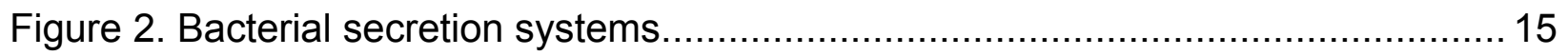

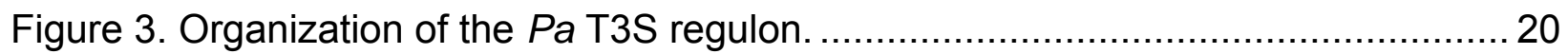

Figure 4. Comparison of flagellar and injectisome structure ...................................... 22

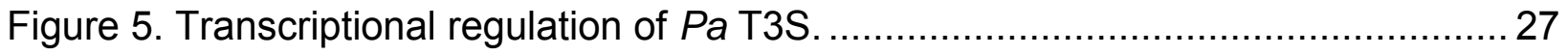

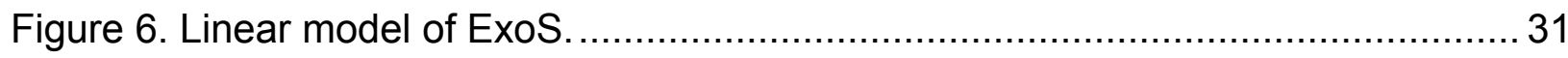

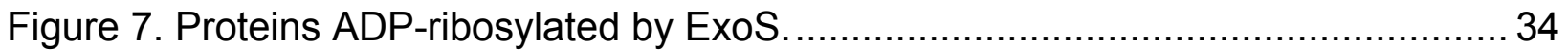

Figure 8. Time course analysis of T3S-T-ExoS substrate modification. ........................ 36

Figure 9. Differential effects of T3S-T-ExoS on DNA synthesis. ................................. 39

Figure 10. Effects of T3S-T-ExoS on epithelial and fibroblastic cell morphology. ..........55

Figure 11. Effects of T3S-T-ExoS on epithelial and fibroblastic cell re-adherence........57

Figure 12. Bacterial association with HT-29, LNCaP, NIH-3T3 (NIH) and Swiss-3T3

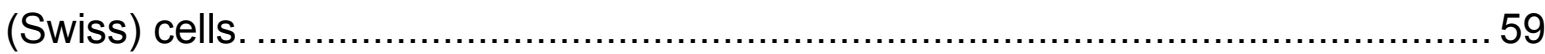

Figure 13. Cell line-related effect of substrate modification by T3S-T-ExoS. ................61

Figure 14. Time course analysis of ExoS substrate modification and internalization of

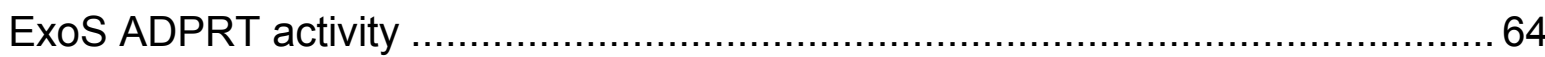

Figure 15. Patterns of LMWG-protein substrate modification by T3S-T-ExoS in different cell lines.

Figure 16. SEM analysis of the morphological effects of ExoS on HL-60 or TPA-HL-60 cells.

Figure 17. ExoS ADPRT substrate modification in HL-60 and TPA-HL-60 cells. .......... 89

Figure 18. Analysis of PopD induction by HL-60 and TPA-HL-60 cells....................... 92

Figure 19. Time-course analysis of T3S-T-ExoS ADPRT activity and substrate

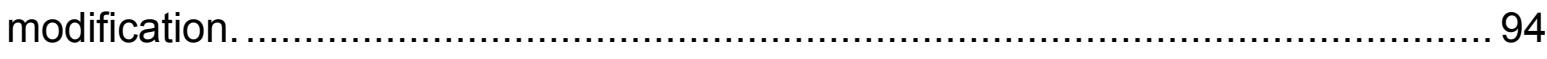

Figure 20. T3S-T of ExoS protein into HL-60 and TPA-HL-60 cells.......................... 96

Figure 21. Effect of MLD, GAP and ADPRT mutations on ExoS ADP-ribosylation of cellular substrates.

Figure 22. Digitonin extraction of eukaryotic cells treated with $\mathrm{Pa}$ expressing wild type or mutant ExoS. 
Figure 23. Effect of MLD on intracellular targeting and localization of T3S-T-ExoS

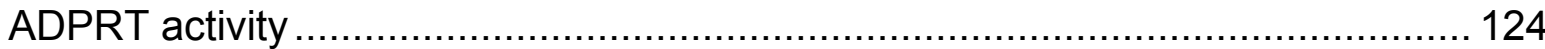

Figure 24. Effect of protease inhibitors on the intracellular targeting and T3S-T of ExoS

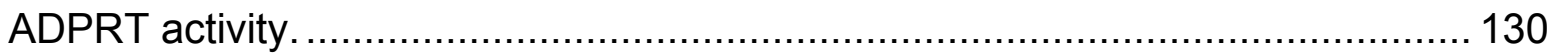

Figure 25. Effect of GAP and ADPRT mutations on the T3S-T of ExoS.................... 133

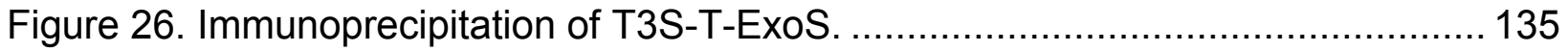

Figure 27. Silver stain analysis of T3S-T-ExoS IP eluates..................................... 137

Figure 28. T3S-T ExoS co-immunoprecipitates with 14-3-3................................. 138

Figure 29. Model of differential targeting of T3S-T-ExoS ADPRT activity .................... 162 


\section{Table of Tables}

Table 1. LMWG-protein substrate modification by T3S-T-ExoS in different cell lines. .. 47

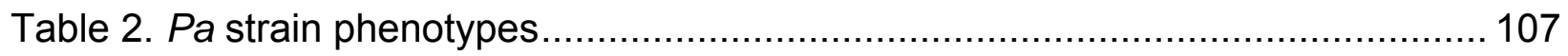

Table 3. Summary of ExoS ADP-ribosylation patterns and subcellular localization of

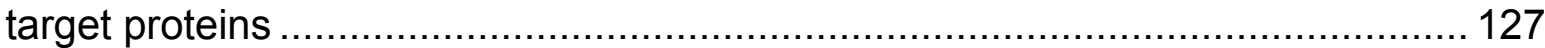




\section{Abbreviations}

388 exs1::Tn1, 388-11

$388 \Delta$ exoS, $\mathbf{3 8 8 \Delta S}$

388 $\Delta p o p D:: T c^{*}, \mathbf{3 8 8 \Delta P o p D}$

Acyl homoserine lactone, AHL

ADP-ribosyltransferase, ADPRT

ADP-ribosyltransferase 5, ART5

American Type Culture Collection, ATCC

4-(2-aminoethyl)benzenesulfonyl fluoride, AEBSF

Bovine serum albumin, BSA

Colony forming units, cfu

Cystic fibrosis, $\mathbf{C F}$

CF transmembrane conductance regulator, CFTR

Diphtheria toxin, DT

DNA/protein macromolecular complexes, T-DNA

Dulbecco's Modified Eagle's Medium, DMEM

Dulbecco's phosphate buffered saline, DPBS

Early endosomal autoantigen 1, EEA1

Elongation factor-2, EF-2

Enhanced chemiluminescence, ECL

$\mathrm{N}$-(trans-Epoxysuccinyl)-L-leucine 4-guanidinobutylamide, E64

Ethylenediamine tetraacetic acid, EDTA

Ethyleneglycol tetraacetic acid, EGTA

Exoenzyme S, ExoS

Exoenzyme T, ExoT

Exoenzyme U, ExoU

Exoenzyme Y, ExoY

Exotoxin A, ETA

Fetal bovine serum, FBS

Guanine nucleotide exchange factor, GEF 
GTPase activating protein, GAP

Glyceraldehyde 3-phosphate dehydrogenase, GAPDH

Glycolipid gangliotetraosylceramide, asialo GM1

Heat shock protein 70, HSP70

Hemagglutinin-tagged ExoS, ExoS-HA

HEPES buffered saline, HBS

Horizontal gene transfer, HGT

Lipopolysaccharide, LPS

Low molecular weight G-proteins, LMWG-proteins

Luria broth, LB

$\mathbf{\alpha}_{2}$-macroglobulin receptor/low density lipoprotein receptor-related protein, $\mathbf{\alpha M R / L R P}$

Matrix assisted laser desorption/ionization mass spectrometry, MADLI-MS

Multiplicity of infection, MOI

Nicotinamide adenine dinucleotide, NAD

Nitriloacetic acid, NTA

Non-T3S-induction medium, TSBD

$100 \mathrm{units} / \mathrm{ml}$ penicillin and $100 \mu \mathrm{g} / \mathrm{ml}$ streptomycin, PS

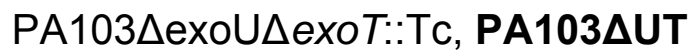

Phorbol-12-myristate 13-acetate, TPA

Protease inhibitor, PI

Protease inhibitor cocktail, PIC

Pseudomonas aeruginosa, $\mathbf{P a}$

Scanning electron microscopy, SEM

Sodium dodecyl sulfate polyacrylamide gel electrophoresis, SDS-PAGE

Soybean trypsin inhibitor, SBTI

SV-40-transformed human WI-38 fibroblasts, WI-38t

TPA-differentiated HL-60 cells, TPA-HL-60

Tris buffered saline, TBS

Triton X-100, TX-100

$0.5 \%$ trypsin- $0.53 \mathrm{mM}$ EDTA, trypsin-EDTA

Two-dimensional electrophoresis, 2DE 
Type I secretion, T1S

Type II secretion, T2S

Type III secretion, T3S

T3S-induction medium, TSBD-N

T3S-mediated translocation/bacterial translocation, T3S-T

T3S-translocated/bacterially translocated ExoS, T3S-T-ExoS

Type IV secretion, T4S

Type V secretion, T5S

Yersinia outer proteins, Yops 
Chapter 1: Literature Review 
Pseudomonas aeruginosa: Pseudomonas aeruginosa $(\mathrm{Pa})$ is a gram-negative rod-shaped bacterium with a single polar flagellum (181). It is a ubiquitous environmental organism, which inhabits terrestrial, freshwater and marine habitats. Soil is thought to be primary ecological niche of $\mathrm{Pa}(83)$, where it plays a role in decay and degradation. Despite its association with life-threatening illness, Pa causes disease only upon gaining access to compromised plants, animals or humans. In the environment, $\mathrm{Pa}$ can colonize plants and healthy animals without disease $(83,118,181)$ and is only overtly cytotoxic to phagocytic amoebae, presumably as a means of self-preservation (84). The success of $\mathrm{Pa}$ as an opportunistic pathogen closely correlates with its versatility as an environmental organism. Correspondingly, genes encoding regulation and environmental sensing proteins make up $8.4 \%$ of its $6.3 \mathrm{Mbp}$ genome (225). Additionally, Pa can utilize many different types of compounds as a source of energy, produce multiple virulence factors, tolerate a wide range of temperatures, and, notably, is intrinsically resistant to a large number of antibiotics $(83,138,140,166,181,248)$.

Since the late 1800's, Pa had been linked to infections of wounds and surgical dressings. However, William Osler was the first to observe that the organisms inhabited previously damaged tissues and were not the primary cause of the infection, thus characterizing Pa as an opportunistic pathogen (181). In the 1960's, Pa emerged as a major human pathogen due to its ability to infect cystic fibrosis and burn patients. Illustrating the opportunistic nature of Pa infections, Donald D. Stieritz and lan Alan Holder developed an experimental burned mouse model (224). Mice were subjected to a nonlethal burn by ethanol flame, and the $\mathrm{LD}_{50}$ for $\mathrm{Pa}$ was determined after subcutaneous injection at the burn site at various time points from 0 hours to 7 days, 
after the initial burn. The $\mathrm{LD}_{50}$ of control mice (mice not sustaining burn injury) was $1.3 \mathrm{x}$ $10^{6}$ organisms. Inoculation of burn sites with fewer than 10 organisms resulted in death of $50 \%$ of the animals tested within the first 18 hours. However, the longer the recovery period (i.e. 24 hours to 7 days) before the tissue was inoculated with $P a$, the closer the $\mathrm{LD}_{50}$ was to control mice. Interestingly, burned mice inoculated with $\mathrm{Pa}$ at nonburned skin sites were the least susceptible to Pa-associated morbidity and mortality, as compared to unburned mice (224). These studies validated the notion that $P$ a causes infections by gaining direct access to damaged tissues. In recent years, $P a$ has been reported to infect neutropenic recipients of chemotherapy, immunosuppressed individuals with AIDS, or drug addictions. However, $\mathrm{Pa}$ infections are primarily nosocomial and typically associated with severe burn victims, persons with cystic fibrosis (CF) and persons who wear contact lenses for extended periods of time (49, $145,181)$.

Pa pathogenesis: Creating a cohesive model of Pa pathogenicity is complicated by the fact that there is no clear phenotypic or genetic delineation between environmental and disease-associated strains $(199,251)$. It is likely that any Pa residing in the natural environment has the potential to cause disease in a compromised individual. There are three stages to Pa pathogenesis (Figure 1): (1) colonization of the compromised tissue, (2) elaboration of virulence factors, and (3) persistence within the host. Synonymous with the ability of $P a$ to colonize compromised tissues is its ability to adhere to eukaryotic cells (253). Both bacterial and host factors are involved in Pa adherence to host tissues. Among the bacterial factors implicated in $\mathrm{Pa}$ binding to damaged tissue, 


\section{A. Healthy airway}

\section{B. Compromised airway}

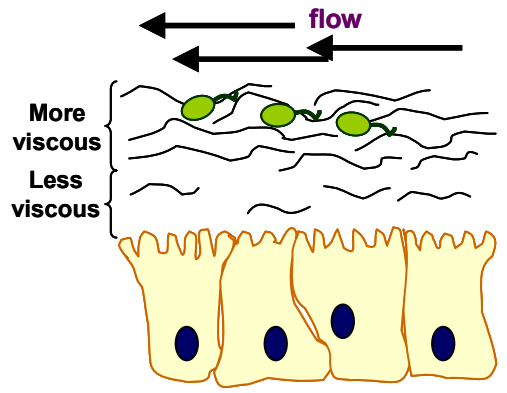

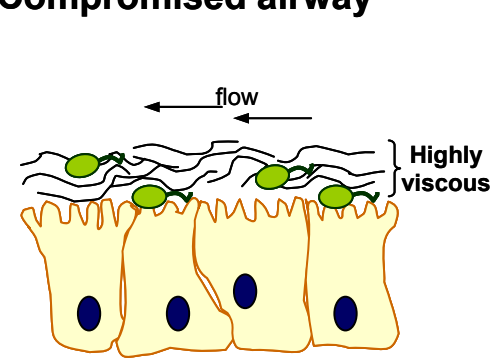

1. Bacterial adherence

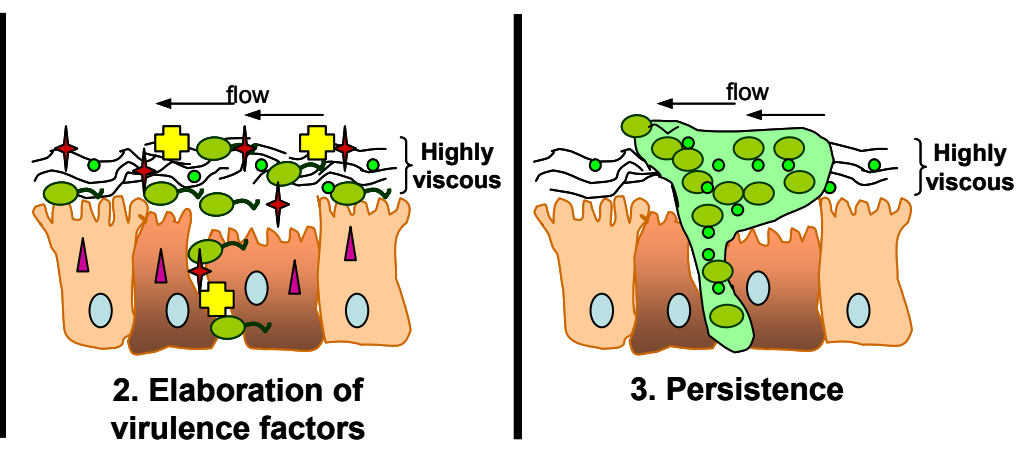

\section{Figure 1. Model of Pa pathogenesis.}

A. In a healthy, non-compromised airway, Pa may bind to the viscous mucus and be cleared. In an alternative hypothesis, $P a$ binds to apical receptors, mainly CFTR, and is internalized. Infected epithelial cells are then desquamated and removed from the lung by the mucus (145). B. Within a compromised airway, depicted here as a CF lung, $P a$ is drawn to the epithelial cells by the viscous mucus. Bacterial adherence is established, which leads to the elaboration of virulence factors. Virulence factors are represented by proteases (yellow crosses), exotoxin A (ETA) (red stars), and T3S effector proteins (purple triangles). Also depicted are acyl homoserine lactones (AHLs) (small fluorescent green circles), when the AHLs reach a threshold density, Pa forms a biofilm, where it persists within the host. Within the biofilm, Pa loses motility as well as the ability to express virulence factors $(103,145,146)$. 
the most thoroughly studied are type IV pili and lipopolysaccharide (LPS) $(185,203$, $207,254,265)$. Specifically, type IV pili and LPS were found to bind purified glycolipid gangliotetraosylceramide (asialo GM1) (85), a receptor found on the apical surface of epithelial cells. There is much debate within the literature about which specific factors are necessary for adherence, and suggestions of a redundancy of $\mathrm{Pa}$ adherence mechanisms or lack of receptor specificity required for bacterial binding.

Given the pathopneumonic nature of $P a$ infections within lungs of CF patients, distinguishing qualities of these tissues were investigated to try to identify host factors which facilitate bacterial binding. Affinity of $P a$ to $C F$ lung tissues has been linked to increased exposure of asialo GM1 $(107,202)$, resulting in increased type IV pili receptors (204). Bolstering this hypothesis, it was found that respiratory epithelial cells recovering from stress or injury expressed greater amounts of asialo GM1 (41). In 1997, Pier et al. hypothesized that the CF transmembrane conductance regulator (CFTR) acts as a receptor that internalizes $\mathrm{Pa}$, thus allowing healthy individuals to clear a $\mathrm{Pa}$ infection by desquamation of infected cells. However, mutant CFTR inhibited both internalization of $\mathrm{Pa}$ and subsequent clearance of $\mathrm{Pa}$, resulting in a persistent infection in the lung (180). Interestingly, internalization of $\mathrm{Pa}$ via CFTR in corneal epithelial cells has been linked to increased infectivity, while mutant CFTR was protective against $\mathrm{Pa}$ corneal keratitis (266). Additionally, studies have found that $P a$ binds fibronectin and vitronectin $(135,187,195)$, concordant receptors $\alpha 5 \beta 1$ and $\alpha v \beta 5$ integrins, as well as other components of basement membranes $(135,195)$. Combined, these findings support an alternative hypothesis that $P$ a readily infects compromised tissue because of greater access to tight junctions and basolateral membranes $(58,59,133)$. 
Recent studies indicate that $P a$ utilizes membrane rafts to facilitate association with eukaryotic cells. Membrane rafts are distinct domains on the plasma membrane that are rich in cholesterol and sphingolipids and serve as dynamic platforms to cluster receptors and connect them to intracellular signaling pathways. Upon purification, membrane rafts are detergent insoluble and of low density, making them easily isolated from the rest of the plasma membrane by differential centrifugation (213). While rafts have been implicated in the pathogenesis of microorganisms (149), it is suggested that the use of membrane rafts by $\mathrm{Pa}$ provides host protection. Studies have shown that $\mathrm{Pa}$ bind to CFTR on membrane rafts and are internalized by epithelial cells. These cells are subsequently sloughed off and $\mathrm{Pa}$ is cleared $(82,121)$. Other contradictory studies have illustrated that $P a$ utilize membrane rafts during infection of type I pneumocytes in lung alveolar sacs and corneal infection $(262,264)$. The lack of a consensus about adhesion strategies used by $\mathrm{Pa}$ to associate with eukaryotic cells is likely illustrative of its versatility and ability to survive in multiple diverse environments.

Once adherence to damaged tissue is established, $\mathrm{Pa}$ elaborates an arsenal of virulence factors to help promote its existence within the new environment. These virulence factors include hemolysin, elastase, alkaline protease, phospholipases, alginate, endotoxin, exotoxin A (ETA), and two type III secretory (T3S) effectors exoenzyme $S$ (ExoS) and exoenzyme $U$ (ExoU) $(3,12,138,140,162-164,212)$. In general, production of these virulence factors promotes the survival of $\mathrm{Pa}$ by causing tissue damage and the release of nutrients, promoting evasion from host immune defenses, and allowing dissemination within the host $(8,138,140,164,174)$. ETA, which is the most toxic $P$ a virulence factor $(138,140)$, is an ADP-ribosyltransferase 
(ADPRT) that targets elongation factor-2 (EF-2) (105). Resultant ETA-ADP-ribosylation of EF-2 inhibits cellular protein synthesis (105).

Environmental queues, such as iron, phosphate, calcium and zinc chloride, influence the expression of multiple virulence factors, including ETA, alkaline protease, elastase, hemolysin and the T3S pathway $(60,139,216)$. For example, in contrast to limited iron levels within the mammalian host, the natural environment provides an abundance of iron, which contributes to the growth and survival of $P a$. Therefore, to survive within the host, $\mathrm{Pa}$ must have mechanisms to scavenge iron. In iron-limiting environments, such as the environment found within the host, Pa produces ETA, alkaline protease, elastase and siderphores. It is hypothesized that ETA and the proteases combine to cause cellular damage, thereby releasing iron for uptake by siderophores (239). To control the release of iron, expression of genes involved in iron acquisition are tightly regulated. The expression of ETA and siderophores are regulated in parallel, but independently of each other, by the repressor, Fur. In ETA regulation, iron-bound Fur negatively regulates the extracytoplasmic sigma factor, PvdS by binding to the promoter region of pvdS. However, in iron-limiting conditions, no repression of pvdS transcription by Fur occurs, ultimately resulting in the expression of ETA $(23,239)$.

While ETA contributes significantly to tissue damage and mortality during $\mathrm{Pa}$ infection, it is recognized that $\mathrm{Pa}$ virulence factors act in concert to promote the survival of the microbe (164). Another virulence mechanism is the T3S pathway, which directly translocates bacterial effector proteins into mammalian cells to alter cellular function. Clinical isolates harboring T3S genes are associated with higher mortality and prolonged infection $(52,200)$. Genetically altered strains of $P a$ that no longer express 
components of the T3S pathway have diminished virulence in the mouse burn model (96). Also in mouse models of infection, immunization against a T3S component, PcrV, which is required for effector translocation, was found to protect infected mice from morbidity and mortality $(95,161,196,208)$. To date there are four identified $\mathrm{Pa}$ T3S effector proteins, ExoS, ExoT, ExoU and ExoY $(55,258,260,261)$. Recent studies have shown that ExoU, an acute cytotoxin and phospholipase $(55,206)$, is the most cytotoxic Pa T3S effector protein (212). ExoS, a bifunctional GTPase activating (GAP) and ADPRT protein $(77,106)$, interferes with clearance from the initial site of infection, and aids in dissemination of the organism (212). Interestingly, clinical and environmental isolates commonly harbor either the exoU or exoS gene, but not both, indicating a mutually exclusive relationship, with exoS being more predominant than exoU $(52,54)$.

While the production of virulence factors is advantageous for establishing infection, persisting within the host requires a different microbial strategy. Pa strains isolated from chronic infections of the CF lung are notably non-motile and T3S- negative $(108,144$, 148). When strains isolated from chronic infection were tested for virulence in mouse models of infection, the majority were found to be avirulent (143). Further examination of characteristics of clinical isolates from chronic infections revealed that microbes exist in chronic infections as biofilms and express the rhl quorum sensing system (214). These studies indicate a selective pressure from the host for $\mathrm{Pa}$ to alter its lifestyle to persist after establishing an infection.

Biofilms are complex differentiated microbial communities. They originate from planktonic bacteria, which adhere as a monolayer to a substratum. These bacteria divide to form microcolonies that combine and differentiate to form fluid-filled 
macrocolonies surrounded by a matrix of polysaccharide and proteins. Pa biofilms are resistant to phagocytosis, host antibodies and antibiotics, and contribute to chronic infections in CF lung and cornea. In addition, biofilms can contribute to $\mathrm{Pa}$ dissemination by regularly shedding single planktonic bacterium (201). The initiation of biofilm formation requires flagella, type IV pili and twitching motility (171). The differentiation of biofilms requires quorum sensing (247). Quorum sensing allows microbes to communicate with one another via diffusible extracellular signals which, in $\mathrm{Pa}$, are acylated-homoserine lactones (AHLs). When cell density is high, the increased concentration of AHLs induces a downstream signaling cascade that leads to the expression of genes responsible for the formation of biofilms (179). While there is evidence that quorum sensing may play a more direct role in the virulence of $\mathrm{Pa}(175$, 201), it ultimately controls expression of components of a persistant infection, including biofilm formation and alginate, which is the polysaccharide component of biofilms (11, 214, 247).

Recent studies have indicated that the inverse relationship between a virulent state and a persistent state is genetically regulated through two main pathways. RetS, which is a hybrid sensor kinase/response regulator, controls signaling pathways involved in increasing the expression of the T3S pathway, type IV pili and ETA (78). In addition, RetS activity inhibits the expression of exopolysaccharide genes involved in biofilm matrix. Mutation of RetS results in hyperadhesion and loss of virulence mechanisms, such as expression of the T3S pathway or type IV pili (78). Acting in opposition to RetS is the GacS/GacA two-component regulatory system, which downregulates expression of virulence factors, such as the T3S pathway and type IV pili, and upregulates 
components essential for biofilm formation $(78,173)$. Also, mutation of genes controlling expression of quorum sensing and biofilm maturation results in an up-regulation of T3Spathway expression $(16,125)$. The environmental signals that control the switch between factors favoring virulence to factors favoring biofilm formation are not yet clear. However, it is a common supposition that the environment created by $\mathrm{Pa}$ after initial infection requires further adaptation to support persistence within the host.

Our studies have focused on the T3S effector, ExoS in Pa pathogenesis. $\mathrm{Pa}$ is not an overt pathogen and will only infect compromised tissues, which indicates that a compromised host environment allows or signals the initiation of an infection. Given the intimate interaction between the bacterium and host cell, which allows the T3Smediated translocation (T3S-T) of ExoS into the eukaryotic cell, we are interested in understanding how the host cell influences not only the translocation of ExoS, but also the intracellular targeting of its activity.

Characterization of ExoS: Classical bacterial toxins are classified by their $A B$ structure and their mechanism of action. The A portion of a toxin includes the enzymatic activity (A subunit), and the B portion includes the cell binding and translocation functions (B subunit). Three main $A B$ toxin structures have been described. ${ }^{1} A B$ toxins are synthesized as a single polypeptide, which includes an A (active) subunit and a B (binding) subunit. Connecting the amino-terminal A subunit to the carboxy-terminal B subunit is translocation or T domain. Once the toxin has bound and been translocated into the cells, the A subunit must be cleaved from the B subunit to express full catalytic activity. ${ }^{2}$ In $A B_{n}$ toxins, the $B$ subunit is synthesized as a separate polypeptide and forms an oligomer which binds to target host cells. The A polypeptide has a high affinity 
for the oligomer and becomes enzymatically active when an internal site is cleaved. In these toxins, the translocation function has not yet been fully described. ${ }^{3}$ Bipartite toxins are comprised of two distinct soluble proteins. One of the proteins is responsible for cell binding and membrane translocation, and the other confers enzymatic activity. Many toxins affect host cell function by targeting and modifying eukaryotic proteins, thereby altering their function (23).

ETA is a classical $A B$ toxin. The single polypeptide is a $71.5 \mathrm{kDa}$ proenzyme that when denatured or proteolytically cleaved produces a $27 \mathrm{kDa}$ enzymatically active fragment (A domain) and a $45 \mathrm{kDa}$ enzymatically inactive fragment (B domain) (238). Further analysis identified 3 distinct structural domains: domains I and II for the B domain, while domain III is the enzymatically active, A domain (4). Domain I, within the $\mathrm{N}$-terminal half of the molecule, forms an antiparallel $\beta$-structure (4) which binds to the eukaryotic cell receptor $\alpha_{2}$-macroglobulin receptor/low density lipoprotein receptorrelated protein $\left(\alpha_{2} \mathrm{MR} / \mathrm{LRP}\right)(120)$. The ETA proenzyme is internalized by the host cell through receptor-mediated endocytosis (57). Once inside a vesicle, domain II, which is composed of mainly alpha helices (4), acts to translocate the processed A-subunit into the cytosol (109). Four sequentially formed disulfide bonds must be cleaved or denatured to form a catalytically active ETA molecule $(4,238)$. Domain III, within the carboxy-terminus, includes the ADPRT activity of ETA (4). Upon translocation to the cytosol, ETA's ADPRT activity targets EF-2, thereby inhibiting host cell protein synthesis (105). ETA illustrates the efficient design of bacterial toxins, each distinct domain confers a distinct function that contributes to the action of the molecule as a whole. 
ExoS was first identified as an ADP-ribosyltransferase distinct from ETA when more radioactive ADP-ribose was transferred in partially purified EF-2 preparation than could be accounted for by the calculated amount of available EF-2. ExoS was characterized in Pa strain 388, which does not produce ETA (106). Comparisons of the ADPRT activity of ETA to that of ExoS defined unique characteristics of ExoS. Activity was not neutralized by anti-ETA antibody and was destroyed by pretreatment with urea (ETA activity is promoted by urea treatment). In addition, ExoS was found to be more heatstable than ETA and did not target EF-2 (106). ExoS was found not to have one preferred eukaryotic substrate, but to ADP-ribosylate several different targets in wheat germ extract and reticulocyte lysates (106). In in vitro reactions, the preferential eukaryotic target proteins of ExoS were found to belong to the Ras family of low molecular weight G-proteins (LMWG-proteins) $(10,28,30,63,65,89,153,190)$, and ExoS was also found to target vimentin (26). In vivo analysis of ExoS ADPRT activity confirmed that ExoS targeted LMWG-proteins, as well as, cyclophilin A and ezrin, radixin and moesin proteins (ERMs) within the eukaryotic cell $(44,63,65,89,150,153)$. In order to ADP-ribosylate target proteins, ExoS requires a eukaryotic cofactor from the 14-3-3 protein family, for catalytic activity $(29,68,92)$.

ExoS purified from culture supernatants of strain 388 was an aggregate of two proteins of 49 and $53 \mathrm{kDa}(106,127,165)$. Originally it was hypothesized that the 53 $\mathrm{kDa}$ protein was a proenzyme form of ExoS, since the 49 and $53 \mathrm{kDa}$ proteins were immunologically cross-reactive $(127,165)$, shared a common amino-terminus $(25)$, and produced common peptides following cleavage with cyanogen bromide and proteases (104). In support of this hypothesis and consistent with toxin structure/function models, 
after extraction from an SDS-polyacrylamide gel only the $49 \mathrm{kDa}$ form displayed ADPRT activity $(29,128,165)$. The common origin of the $49 \mathrm{kDa}$ and $53 \mathrm{kDa}$ proteins was supported by their highly regulated coordinate expression. The production of both the $49 \mathrm{kDa}$ and $53 \mathrm{kDa}$ proteins by the bacterium increased or decreased in parallel with changes in the culture medium $(25,104)$. Medium promoting maximal production contained a chelator, nitrilotriacetic acid (NTA), ethylenediamine tetraacetic acid (EDTA) (231) or ethyleneglycol tetraacetic acid (EGTA) (60). The production of both proteins was inhibited by the addition of calcium or zinc chloride salts (60).

The cumulative data indicated that the $49 \mathrm{kDa}$ and $53 \mathrm{kDa}$ proteins were related, but confirmation of their being two forms of the same protein was lacking. A genetic approach was employed to explore if the proteins were produced by the same gene. For this, Pa strain PA103, which produced the $53 \mathrm{kDa}$ protein, but not the enzymatically active $49 \mathrm{kDa}$ protein (62) was transformed with the $49 \mathrm{kDa}$ structural gene cloned from strain 388 and cultured under inducing conditions. Both the $49 \mathrm{kDa}$ and $53 \mathrm{kDa}$ proteins were produced in response to NTA (126). In addition, the allelic exchange of the $49 \mathrm{kDa}$ ExoS gene with a tetracycline gene cartridge in strain 388 (forming strain $388 \Delta$ exoS::Tc), eliminated the production of $49 \mathrm{kDa}$ ExoS, but the production of the 53 $\mathrm{kDa}$ protein was unaffected (126). This was the first data that supported that $49 \mathrm{kDa}$ ExoS and the $53 \mathrm{kDa}$ protein were encoded by separate genes. An open reading frame, termed exoT, was identified by Southern blot analysis of $388 \Delta$ exoS::Tc, using exoS to probe chromosomal digests (256). The exoT gene product was identified as the $53 \mathrm{kDa}$ protein, first known as Exo53, then later as ExoT. While ExoT shares $75 \%$ amino acid identity to ExoS, the recombinant form of this protein displayed only $0.2 \%$ ADPRT 
activity $(256,260)$. Together, these studies confirmed that $49 \mathrm{kDa}$ ExoS and $53 \mathrm{kDa}$ ExoT were distinct proteins, and that the $53 \mathrm{kDa}$ protein was not a proenzyme form of ExoS.

In determining the role of ExoS during the $\mathrm{Pa}$ infection process, a transposon $\mathrm{Tn} 1$ mutant of strain 388, lacking ADP-ribosyltransferase activity, was generated and found to be 2,000 -fold less virulent than the parent strain 388 (165). In an experimental mouse burn wound model, using strain 388 and the null ExoS strain (388exs1::Tn1), the production of ExoS did not alter the ability of $\mathrm{Pa}$ to colonize the site of the wound. However, without ExoS, the organism was compromised in the ability to disseminate, implicating ExoS at this stage of the infection process $(162,165)$. Yet, conflicting with evidence that ExoS played a role in the virulence of $\mathrm{Pa}$ was the fact that purified ExoS was not toxic to animals $(162,163)$ nor to cultured eukaryotic cells $(25,170)$.

Additionally, culture supernatants obtained from ExoS-producing bacteria were nontoxic to cultured cells $(7,170)$. These data suggested that ExoS did not have a structural B domain that allowed its binding or translocation into eukaryotic cells. During the mid1990's a common conclusion was that delivery of ExoS to eukaryotic cells was by a unique mechanism, not yet described for other toxins.

Bacterial secretion systems: To date, there are five main processes described for a Gram-negative bacterium to secrete or transport a protein from the bacterial cytoplasm and across the inner membrane, periplasmic space and outer membrane (Figure 2) (88). These processes are grouped based on their dependence on the Sec pathway of protein export. The Sec pathway, also known as the general secretory 
Sec independent

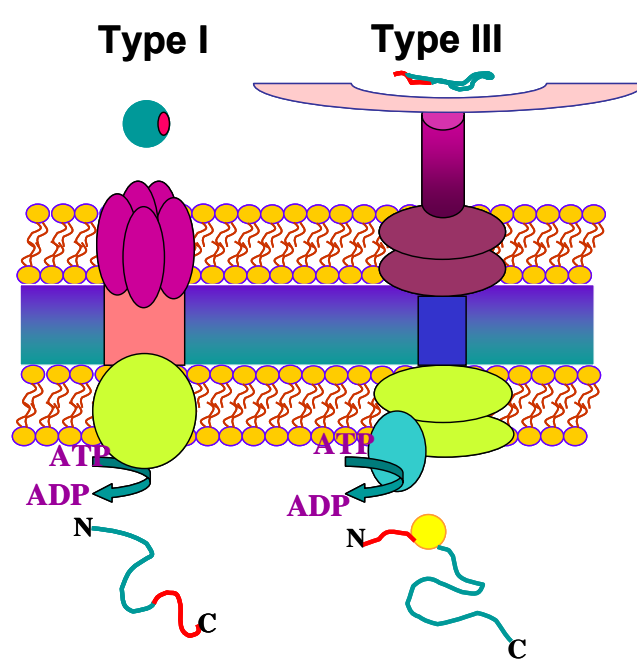

Sec dependent

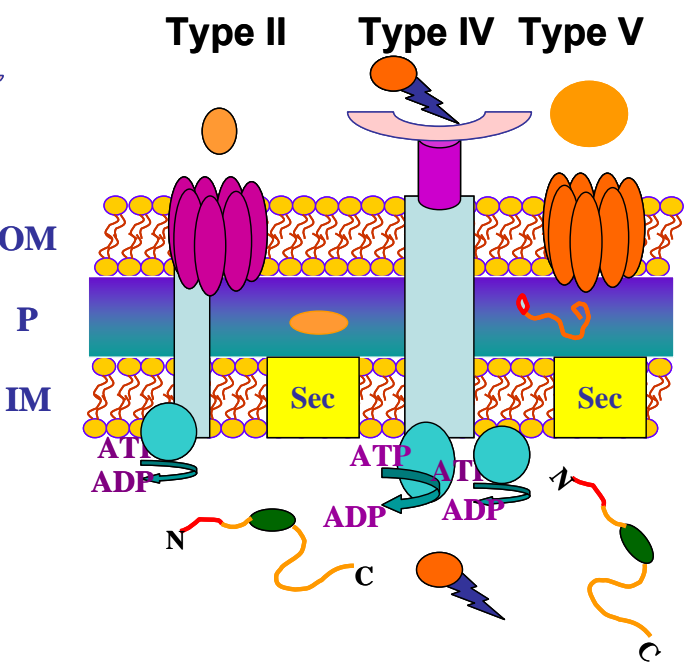

\section{Figure 2. Bacterial secretion systems.}

Grouped by their dependence on Sec proteins for translocation of proteins into the periplasmic space, types I and III secretion pathways involve the one-step secretion of proteins, driven by inner membrane ATPases. Uncleaved signal sequences are found in the C-terminal end of proteins destined for the type I pathway, while signal sequences within the N-terminus identify proteins for secretion by the type III pathway. The type III pathway also differs in that it translocates bacterial proteins across eukaryotic cell membranes. Proteins destined for type II and V pathways contain cleavable N-terminal consensus sequences. The proteins are processed in the periplasmic space before final translocation across the outer membrane. Type $\mathrm{V}$ secreted proteins are known as autotransporters and they oligomerize to form a pore, allowing self-translocation across the outer bacterial membrane. Type IV secretion uses a Sec-dependent process to translocate DNA-protein complexes and large protein complexes either outside the bacterial cell or, via a pilus-like structure, inside the eukaryotic cell. The figure is based on text by Stathopoulus et al., 2000 (219) and Henderson et al., 2004 (88). OM, outer membrane; $\mathrm{P}$, periplasmic space; IM, inner membrane. 
pathway, exports proteins from the bacterial cytoplasm into the periplasmic space, utilizing an inner membrane ATPase and a chaperone protein that binds the presecretory protein. Sec-dependent transport processes are types II, IV and V, while Sec-independent transport processes are types I and III. All pathways are highly conserved among Gram-negative bacterium $(88,205)$.

Type II secretion (T2S) occurs in two steps and requires that the secreted proteins have a concensus signal sequence within the first 30 amino-terminal amino acids, which targets proteins for Sec inner membrane transport. This signal sequence is then cleaved within the periplasmic space and the protein becomes partially folded. Transport across the outer membrane requires a multimeric outer membrane component termed secretin $(88,102,205)$. T2S was first described as the secretion mechanism that Klebsiella aerogenes used to transport pullulanase $(113,230)$. Homologous systems were later identified for other Gram-negative bacteria (205). ETA is an example of a Pa virulence factor secreted via the type II secretory pathway (249).

The type IV secretion pathway (T4SP) is the least understood of the secretory processes, but appears to be closely related to ancestral bacterial conjugation systems. The T4SP allows the transfer of DNA/protein macromolecular complexes (T-DNA) from the bacterium to the host cell $(22,88,249)$. In this regard, the best characterized T4SP is in Agrobacterium tumefaciens which utilizes membrane transport protein VirB to secrete a single-stranded DNA molecule across bacterial membranes and into plant cells, where the DNA integrates into the genome, ultimately resulting in tumor growth (269). T4SP also allows the secretion of large oligomeric protein complexes, such as the large $A-B_{5}$ pertussis toxin (243). It is now thought that secretion of T-DNA occurs in 
one step, while the transfer of protein complexes occurs in two steps and utilizes the Sec inner membrane secretion system $(22,88)$. The two seemingly divergent transport pathways are classified together because the related transporters share architectural similarities to VirB (183).

The type $\mathrm{V}$ secretion pathway (T5SP) describes the secretion mechanism of a group of proteins known as autotransporters. Proteins secreted by this pathway exhibit an extended $\mathrm{N}$-terminal signal sequence that identifies the proprotein for secretion by the Sec inner membrane translocation machinery. Once in the periplasmic space, the signal sequence is cleaved and the remaining $\mathrm{N}$-terminal portion of the molecule forms a $\beta$-barrel structure that inserts into the bacterial outer membrane to form a pore. The Cterminal active portion of the molecule either remains attached to the pore by the linker region or is cleaved upon secretion. It is thought that the folding of the $\beta$-barrel pore structure provides the energy to translocate the active portion from the periplasmic space across the outer membrane (88).

Type I secretion (T1S) is Sec-independent and is a single-step continuous process from the bacterial cytoplasm across both inner and outer membranes. Proteins secreted by this pathway are not processed nor do they have a periplasmic intermediate. The secretion signal sequence is located within the C-terminal 60 amino acids. The type I secretion complex includes an inner membrane ATPase, a channel forming membrane fusion protein and an outer membrane pore. Examples of proteins secreted by this process include $E$. coli alpha-hemolysin and $P a$ alkaline protease $(88,102,205,219)$.

T3S, which is also Sec-independent, displays similarities to both types I and II secretion. T3S was recognized in the early 1990's in association with pathogenicity 
determinates of Yersinia enterocolitica termed Yersinia outer proteins (Yops) (156). Effector proteins secreted by this pathway do not require processing, but include a signal for secretion within the first 10 to 15 residues $(5,88,102,142,205)$. The T3S secretion apparatus forms a needle-like syringe, termed the injectisome, which translocates bacterial effector proteins in one continuous step from the bacterial cytoplasm across the inner and outer membranes, followed by the eukaryotic host cell membrane into the cytosol. Proteins that form the injectisome are conserved among Gram-negative bacteria and are thought to have common evolutionary origins with flagella $(102,147,155,205)$. T3S-effector proteins are bacterium specific, thus allowing different bacteria to manipulate the host cell according to its unique survival needs.

Characterization of $\mathrm{Pa}$ ExoS secretory mechanism: Since its discovery in the late 1970's, the biochemical properties of ExoS have been well studied, but how ExoS ADPRT affected eukaryotic cells remained a mystery, because the purified protein was not toxic when added to cultured cells or administered to animals $(25,162,163,170)$. Comparison of genes affecting ExoS production with those involved in Yersinia Yops production helped to understand how ExoS might be affecting host cells. ExoS did not include an $\mathrm{N}$-terminal consensus signal or $\mathrm{N}$-terminal processing sequences $(128,256)$, indicating that the secretion of ExoS was Sec-independent. Mutations within the carboxy-terminus and internal sequence of ExoS, that would be predicted to alter T1S, did not alter ExoS secretion (258). However, the first nine amino-terminal residues were necessary for ExoS secretion. The activation of a gene encoding the ExsA protein was also absolutely required for ExoS expression. ExsA acts as a trans-regulatory protein and contains a DNA-binding helix-turn-helix motif (61). ExsA is homologous to AraC of 
Escherichia coli and VirF/LcrF (two terms referring to the same protein) of Yersinia enterocolitica $(61,99,257)$. VirF/LcrF controls the expression of $Y$. enterocolitica Yops $(93,130,155)$. Dnase I-footprint analysis of promoters controlled by ExsA found the concensus binding site of ExsA to be similar to the concensus binding sites of VirF (99). A map depicting the organization of the $\mathrm{Pa}$ T3S-regulon in Figure 3 shows the clustering of genes involved in T3S, ExsA binding sites, and the non-contiguous locations of genes that encode the effector proteins, ExoS, ExoT, ExoY, ExoU (Figure 3).

The expression of the ExoS operon in vitro requires growth of $\mathrm{Pa}$ in a cationdepleted medium, similar to the medium required to promote production of Yops (231), indicating similarities between the regulation of ExoS and Yops. The amino-terminus of ExoS displays sequence homology to a secreted effector protein, YopE, indicating that similarities between the Yops and ExoS systems extended beyond regulation (259). In addition, a locus containing an ExsA-dependent promoter and encoding gene products termed PscB-L, was identified and found to be homologous to the YscB-L proteins of Yersinia (258). Mutations within the pscC gene prevented secretion of ExoS. PscC is homologous to a Yersinia protein, YscC, which is involved in T3S of Yops (258). The regulation and homology data suggested that ExoS was secreted by the T3S pathway (258). Therefore, it was recognized that ExoS can only affect eukaryotic cell function if translocated into the host cell after bacterial contact. 


\section{Components of the Pseudomonas aeruginosa Type III Secretion System}

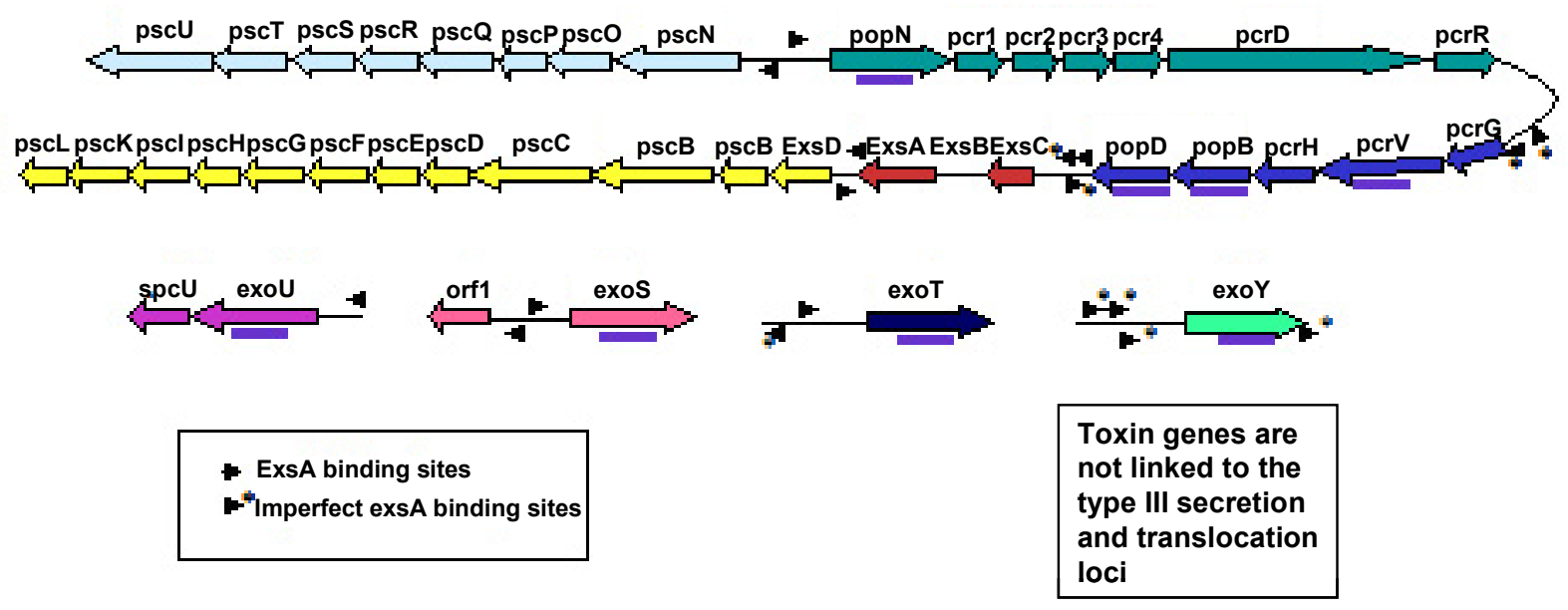

\section{Figure 3. Organization of the $\mathrm{Pa}$ T3S regulon.}

The individual gene products of the Pa T3S system and effector proteins, ExoU, ExoS, ExoT and ExoY are represented by colored arrows. Different colors identify operons based on promoter mapping, transcriptional fusion, or DNA-binding analyses. The effectors and known chaperones (ExoU and SpcU; ExoS and Orf1; ExoT; ExoY) are located at different sites within the chromosome, and are separate from genes encoding proteins involved in the regulation and formation of the T3S apparatus. ExsA consensus and imperfect binding sites are indicated. This figure was published in the book entitled Bacterial Protein Toxins, (ed. Drusilla L. Burns, Joseph T. Barbieri, Barbara H. Iglewski and Rino Rappuoli, ASM press, Washington D.C., 2003). Permission to use the figure was given by ASM press and Dara Frank, who developed the original figure. 
Structural analysis of the T3S process: The conserved nature of the T3S process among pathogenic Gram-negative bacteria is consistent with genes encoding the T3S pathway being obtained either through horizontal gene transfer (HGT) or from a common ancestor. In support of the HGT hypothesis, genes encoding T3S systems reside within plasmids or as gene clusters located in pathogenicity islands, bounded by the remnants of insertion sequences, bacteriophage genes or transposable elements. Pathogenicity islands are also recognized by differences in $\mathrm{G}+\mathrm{C}$ content when compared to the entire bacterial chromosome $(70,102,249)$. In contrast to the T3S regulons of other pathogenic bacteria, the $\mathrm{G}+\mathrm{C}$ content of the genes encoding the $\mathrm{Pa}$ T3S regulon (67.3\%), is similar to the $\mathrm{G}+\mathrm{C}$ content of the entire Pa genome $(69 \%)$, indicating that $\mathrm{Pa}$ T3S regulons do not reside on a pathogenicity island (60). This finding is consistent with the hypothesis that T3S is not just involved in pathogenesis but an integral Pa survival mechanism.

Translocation of proteins via the T3S pathway requires the bacterium and eukaryotic cell to be in close contact, allowing insertion of the bacterial T3S-needle-like apparatus into the host cell membrane (Figure $4 \mathrm{C}$ ). The result is the delivery of bacterial effector proteins directly into the host cell cytosol, allowing the bacteria to strategically hijack host cell processes $(70,76,102)$. Purified T3S complexes from Salmonella typhimurium and Shigella flexneri resemble needles $(18,124)$. Similarities between flagella and the T3S apparatus are evident both structurally and based on protein homology (Figure 4 A and B) $(19,76,147)$. The base of the S. typhimurium needle complex was observed to be similar to that of a flagellar basal body, with two 

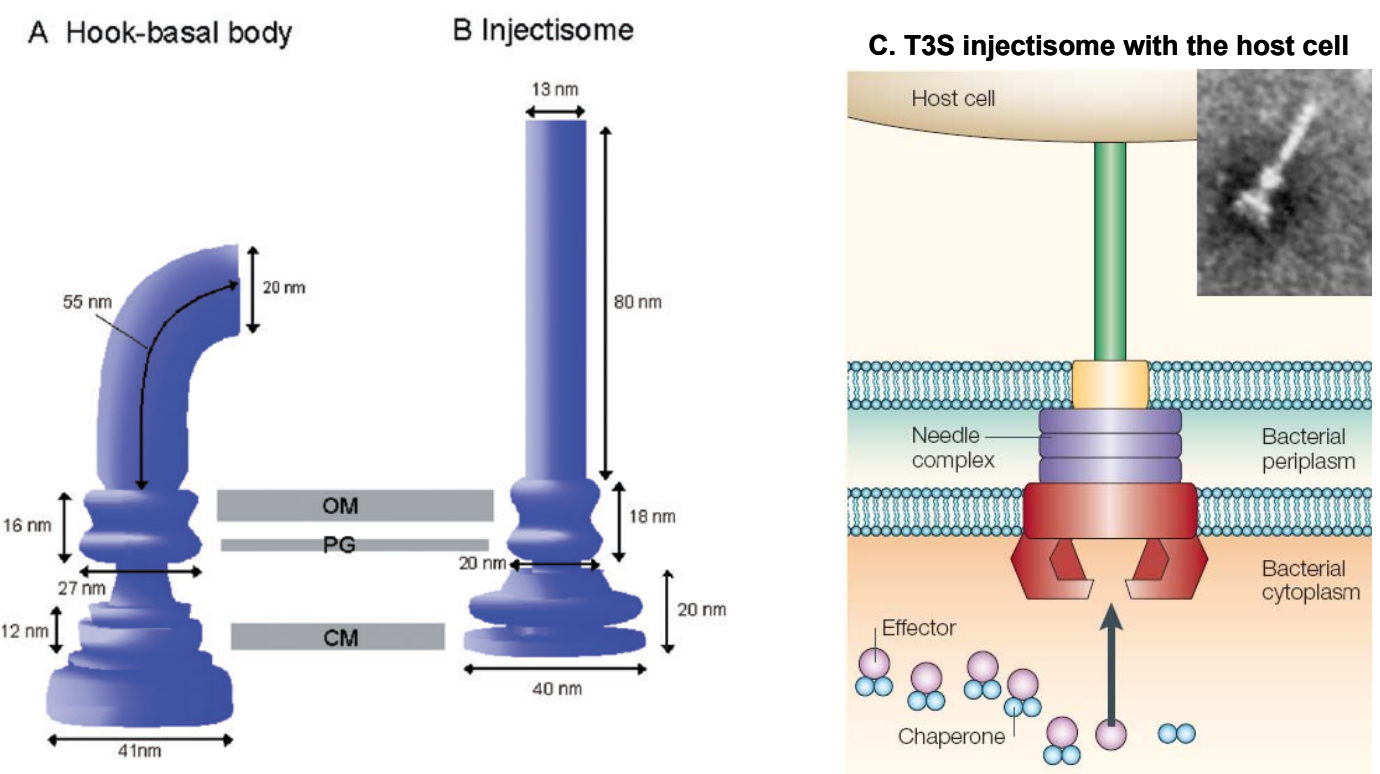

Figure 4. Comparison of flagellar and injectisome structure.

(A) Depicts the dimensions of the flagellar hook-basal body and (B) depicts the T3S apparatus or injectisome of Salmonella. CM, bacterial cytoplasmic membrane; PG, peptidoglycan layer; OM, bacterial outer membrane. (C) Depicts a T3S apparatus in contact with the eukaryotic cell. The needle is drawn based on electron microscopy images (see inset), with several ring structures spanning the inner and outer membranes. The needle filament (green protrusion) is thought to engage a bacterial pore-forming complex (translocator) that becomes lodged in the eukaryotic host cell membrane. The diagram also depicts effector proteins, awaiting translocation, associated with their cognate chaperones.

Parts $A$ and $B$ of the figure were originally published in Molecular Microbiology (2005, 56(2): 303-308) in an article entitled, "Measure for measure in the control of type III secretion hook and needle length", authored by Tohru Minamino and Anthony P. Pugsley. The image appears by permission of Blackwell Publishing. Part $C$ of the figure was originally published in Nature Reviews Molecular Cell Biology (2003, 4: 738-743) in an article entitled, "Priming virulence factors for delivery into the host", authored by $\mathrm{C}$. Erec Stebbins and Jorge E. Galan. The image appears by permission of Nature publishing and C. Erec Stebbins. 
upper and two lower rings (124), with the needle portion of the complex resembling the flagellar hook $(19,76,147)$.

Interestingly, the proteins forming the needle base of the T3S process are localized and positioned within the bacterial inner membrane, periplasmic space and outer membrane in a Sec-dependent manner $(116,228)$. Further assembly of the needle complex requires this basal body structure. Similar to the energy requirements of functional flagella, treatment with sodium azide, which abolishes oxidative phosphorylation, also inhibits T3S (17). The protein that makes up the outer membrane ring of the T3S apparatus differs from the outer membrane ring of flagella. This component, InvG, initially characterized in S. typhimurium, is a member of the secretin family of proteins (116) (InvG is labeled OM in Figure 4B and is depicted by the yellow box in Figure 4C). Secretins usually function alone and are involved in the transport of large macromolecular structures, including the T2S of type IV pili components (137). However, InvG serves a different purpose in the T3S process as it is associated with 2 other proteins consisting of the needle complex basal body $(116,228)$. InvG is homologous to the $\mathrm{Pa}$ T3S protein, $\mathrm{PscC}$, and Yersinia T3S protein $\mathrm{YscC}(60,76)$. While the exact location of PscC within the Pa T3S apparatus is currently unknown, YscC has been found to form a stable oligomeric complex, which forms a channel in the bacterial outer membrane (119). Pa strains lacking a functional $p s c C$ structural gene do not secrete T3S effector proteins (258).

The needle component, first visualized by electron microscopy in S. typhimurium, is thinner than a flagellar organelle, only 7 - to 8 - $\mathrm{nm}$ wide, and $60 \mathrm{~nm}$ long (124). However, the needle-length was found to be organism specific (76). Much like a component of the 
flagellar hook apparatus, T3S needle complexes have a protein that determines the length of the needles. These proteins lack sequence homology to the flagellar protein, FliK, but are functional equivalents in that their mutation or deletion result in T3S needles of variable length, with only shorter needles found incapable of T3S-T (263). A recent study of the $Y$. enterocolitica needle proposes that T3S needle length is related to the distance between bacterium and host cell created by adhesion molecules attaching bacteria to the host cell (159).

Once the needle is assembled, certain bacteria, including S. flexneri, Yersinia, and $\mathrm{Pa}$, secrete proteins termed translocators, which form the end of the needle and insert a channel into eukaryotic membranes (76). In S. flexneri IpaB and IpaC insert into the host cell membrane comprising a channel for the translocation of Shigella effector proteins (17). IpaB and IpaC share structural similarities to Yersinia proteins YopB and YopD, respectively (17). YopB and YopD direct the translocation of effector proteins across the host cell membrane and are functionally interchangeable with $P$ a proteins, PopB and PopD (67). In Pa T3S, PopD was found to be required for the transport of ExoS across $\mathrm{CHO}$ cell membranes. The lack of PopD resulted in secretion of effector proteins from the bacterium, without translocation into eukaryotic cells (236). Attempts at detecting PopD in eukaryotic cell membranes during a Pa infection PopD have been unsuccessful. However, recently PopD was shown to bind to ghost erythrocyte membranes and form oligomers and ring structures in cholesterol-rich liposomes, indicating its potential to form pores in eukaryotic cell membranes $(81,210)$.

Assembly of the needle structure precedes the translocation of effector proteins. The signal sequence targeting effectors for T3S is within the amino-terminus. However, 
whether the source of the secretion signal is an amino acid or mRNA sequence remains controversial. Deletion of the first 15 amino acids of T3S proteins abolishes secretion $(155,218,250)$, which is consistent with either the first 15 amino acids or the mRNA encoding this region being required for secretion. Analysis of T3S-secretion of $Y$. entercolitica effector proteins revealed a role for mRNA sequence in targeting effectors for T3S. Construction of frameshift mutations, which would alter mRNA codons translation, but not the actual mRNA sequence in the sequence encoding YopQ, did not block T3S (6).

Proteins translocated shortly after needle assembly are thought to be translated and sequestered in the bacterial cytoplasm by a chaperone protein. In Salmonella, it has been shown that chaperones maintain T3S effector proteins in a partially unfolded confirmation, while protecting them from aggregation and degradation (220). The ability of effector proteins to unfold prior to translocation, excludes other cytoplasmic proteins from T3S transport (51). However, not all effector proteins have known cognate chaperones. This is true of $\mathrm{Pa}$ in which ExoS and ExoU have chaperones Orf1 and SpcU, respectively, while ExoT and ExoY do not $(56,60)$. Previous studies have indicated that T3S effectors have different efficiencies when binding to chaperones, thereby, allowing for certain effectors to be translocated before others (20). Additionally, it has been reported that there is a translation hierarchy of T3S effectors. For example, blockage of the $Y$. enterocolitica T3S pathway resulted in translation of YopE, but not YopQ, supporting evidence that YopE is T3S-T earlier than YopQ (6). In summary, effectors which are T3S-T early after needle assembly seem to rely on interaction with a chaperone protein, while effectors which are T3S-T later are shuttled to the T3S 
apparatus based on their $\mathrm{N}$-terminal mRNA sequence (19). A hierarchy of secretion underscores the highly regulated manner in which bacteria can manipulate eukaryotic cells to their benefit.

Regulation of Pa T3S. Over 40 genes, which encode regulators, apparatusstructure proteins, translocator proteins and effector proteins, are involved in the T3S pathway $(60,70,102)$. Expression of the $\mathrm{Pa}$ T3S pathway responds to environmental factors such as low calcium, presence of serum or contact with the eukaryotic host cell $(60,236)$. However, recent work has shown that some components of the Pa T3S pathway are constitutively expressed at basal levels, but further induced by appropriate environmental signals (252). Expression of the $\mathrm{Pa}$ T3S is also controlled by global regulators that sense environmental changes, including adenylate cyclase and cyclic AMP regulators $(2,215,227,245,252)$, fimL , a member of a chemosensory pathway (246), and retS, a hybrid sensor kinase/response regulator (78).

At the level of transcription, $\mathrm{Pa}$ T3S is controlled by an intricate network of bacterial cytoplasmic regulators, ExsA, ExsD, ExsC (Figure 5) $(40,61,152)$. ExsA is a transcriptional activator that promotes the expression of $\mathrm{Pa}$ T3S genes $(60,61,257)$. Prior to induction of a functional T3S apparatus, ExsA is bound to ExsD, thus repressing ExsA activity. Deletion of exsD results in constitutive expression of T3S genes. This occurs even in the presence of a mutation affecting outer membrane protein PscC, which prevents the formation of a functional T3S apparatus (152). Also indicating another layer of T3S regulation, mutation of exSC results in very low T3S expression $(40,79)$. 


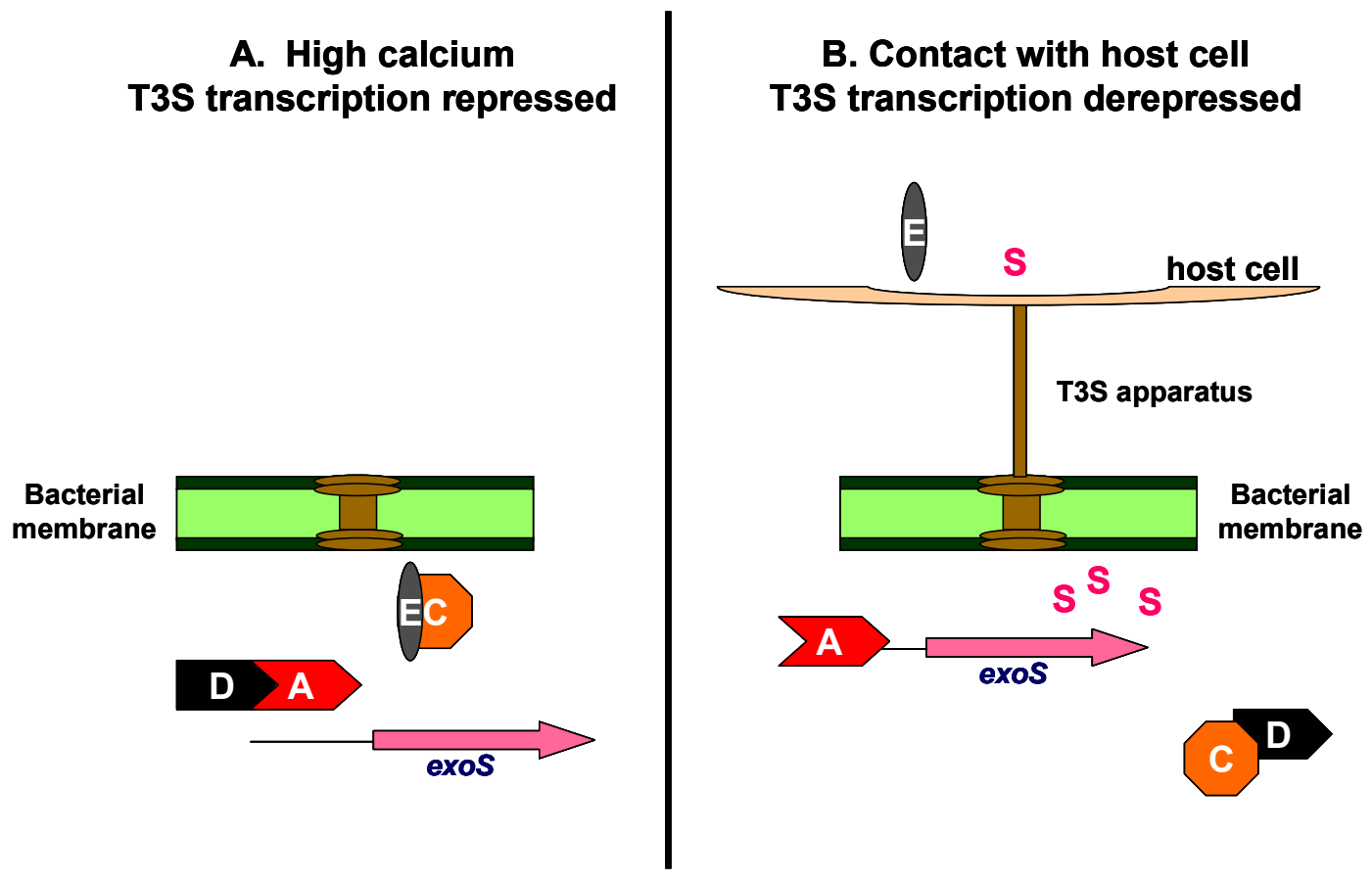

Figure 5. Transcriptional regulation of $\mathrm{Pa}$ T3S.

Transcription of $\mathrm{Pa}$ T3S is activated by ExsA. In conditions that do not favor T3S, such as high calcium (depicted in A), ExsD sequesters and prevents ExsA from activating transcription of T3S genes, such as exoS. In T3S-favorable conditions (depicted in $B$ ), ExsC recruits ExsD from ExsA, freeing ExsA to initiate transcription of T3S genes. ExsA activity is further regulated by ExsE, which binds to ExsC in T3Sunfavorable conditions, preventing ExsC from recruiting ExsD. ExsE is thought to be the molecular sensor of T3S-apparatus readiness. T3S-T of ExsE frees ExsC to interact and recruit ExsD from ExsA. This diagram is based on work by Mark L. Urbanowski, Guinevere L. Lykken and Timothy L. Yahr in a paper entitled "A secreted regulatory protein couples transcription to the secretory activity of the Pseudomonas aeruginosa type III secretion system", which appeared in Proc. Natl. Acad. Sci. USA in July 2005 (102 (28): 9930-9935. 
Further analysis indicated that overexpression of exsC mimicked the deletion of exsD, and that ExsC binds to ExsD. These data indicated that in favorable conditions, ExsC binds to ExsD, thereby freeing ExsA to activate transcription of other T3S genes (40). ExsC exhibits characteristics of a T3S chaperone in that it has a low molecular mass, an acidic isoelectric point, and a carboxy-terminal amphipathic alpha-helix $(51,172)$.

This suggested the possibility that in unfavorable conditions for T3S, ExsC is bound to another protein, which is secreted or translocated upon favorable T3S conditions, thereby freeing ExsC to bind ExsD and ultimately free ExsA (40). Interestingly, immediately downstream of exsC is an open reading frame that encodes an $8.7 \mathrm{kDa}$ protein, termed ExsE. Recent studies have shown that ExsC binds to ExsE, and ExsE is secreted from the bacteria in low calcium conditions $(191,234)$. However, the absence of ExsE does not have any affect on the regulatory activity of ExsD, indicating that there are multiple regulatory checkpoints in the induction and expression of $\mathrm{Pa}$ T3S (234).

Manipulation of the host cell by T3S effector proteins: The T3S-injection of effector proteins into the eukaryotic cell provides a mechanism for Gram-negative bacteria to manipulate host cell function in a manner that promotes their specific lifestyle. While the structure of the T3S-apparatus is conserved among Gram-negative bacteria, effector proteins are species specific. To evade the immune system, Yersinia T3S-translocates multiple effector proteins which coordinately inhibit inflammation and phagocytosis, while inducing macrophage apoptosis $(36,37)$. In contrast, the T3S-T of Salmonella effectors promotes bacterial invasion into the host cell, thus allowing intracellular propagation (37). Underlying the broad effects of T3S-T effectors on 
eukaryotic cells is the intricate manner in which they hijack host cell signaling and mimic the activities of eukaryotic proteins (221).

One example of eukaryotic cell mimicry involves a group of T3S effectors which function similarly to eukaryotic Rho GTPase activating proteins (GAPs). G-proteins are molecular switches, which cycle between active, GTP-bound, and inactive, GDP-bound states. GAPs control G-proteins by accelerating the hydrolysis of phosphate from GTP, forming an inactive GDP-bound-G-protein (237). Rho GAP proteins target Rho, Rac and Cdc42, proteins that control the actin cytoskeleton network $(229,237)$. T3S effector proteins that have been found to contain Rho GAP activity include: Yersinia YopE (242), Pa ExoS and ExoT $(77,122,178)$, and Salmonella SptP (69). Consistent with their GAP activities targeting Rho, Rac and Cdc42, the transfection or T3S-T of YopE, ExoS and ExoT is associated with cell rounding/actin cytoskeleton rearrangement and antiphagocytic effects $(38,66,74,178,192,242)$.

An example of multiple T3S effectors coordinately manipulating host cell function was first illustrated with Salmonella typhimurim proteins, SptP and SopE. Epithelial cells lining the gastrointestinal tract do not normally engulf bacteria. However, Salmonella must enter these epithelial cells to evade the host immune system and initiate the infectious process. T3S-T of SopE activates Rac and Cdc42, resulting in localized actin reorganization and membrane ruffling, which stimulates the host cell to engulf the organism $(45,86)$. Following internalization, S. typhymurium resides within a specialized vacuole. To protect its new niche, the T3S effector SptP acts as a GAP towards Rac and Cdc42, thereby reversing the effects of SopE and allowing the host cell's actin cytoskeleton to recover $(45,69,221)$. The sequential action of SopE and SptP activities 
highlights the sophisiticated manner in which bacteria are able to usurp host cell signaling pathways.

Structure/function relationships of T3S-T-ExoS. While ExoS was characterized as an ADP-ribosyltransferase in 1978 (106), further examination of the functional domains revealed that the $\mathrm{N}$-terminal region of ExoS includes a GAP domain, which targets Rho GTPases (77), while the ADPRT activity resided within the C-terminal portion of the molecule (117). Arginine at position 146 (R146) was found to be required for ExoS GAP activity (77). The co-crystal structure of the N-terminal GAP domain of ExoS with Rac1 revealed that the positioning of the R146 mimics that of catalytic arginines found in eukaryotic GAP molecules without sharing structural homology (255). Two glutamic acid residues control ExoS ADPRT activity. In vitro kinetic analysis found the glutamic acid at position 379 (E379) to modulate the transfer of the ADP-ribose moiety to the target eukaryotic protein, and the glutamic acid at position 381 (E381) to confer the NAD glycohydrolase activity (186). In vitro analysis also found that mutation of the E381 catalytic residue inhibited in vitro ExoS ADPRT activity (186). As previously indicated, the first nine amino acids are required for T3S-T (258). Additionally, ExoS contains a membrane localization domain (MLD) between residues 52 and 71 (176). Studies of Petterson et. al. support that T3S-T-ExoS is processed at a site within the first 78 residues upon translocation into the eukaryotic cell (177). For maximal ADPRT activity, ExoS requires interaction between amino acids 420-429 and a eukaryotic cofactor, 14-3-3 protein (90). The functional regions of ExoS are depicted in Figure 6. 


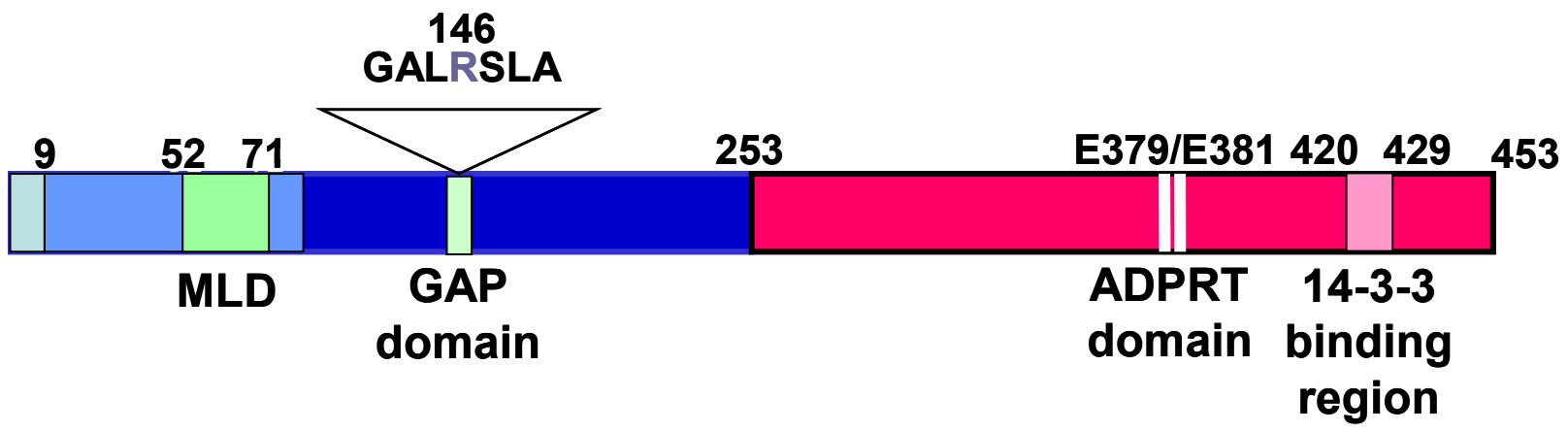

Figure 6. Linear model of ExoS.

ExoS contains 453 amino acids and consists of two separate catalytic domains. Within the N-terminal region is the GAP activity, which targets Rho, Rac and Cdc42 (77). The ADPRT activity is within the C-terminal region (117). The first nine amino acids of ExoS are required for T3S-translocation (258), and the region between amino acids 52 and 71 (MLD), targets ExoS to eukaryotic membranes $(176,188)$. ExoS binds its cofactor 14-3-3 between amino acids 420-429 (90). 
Since the effects of ExoS on cell function can only be realized upon T3S-T, a coculture system was developed in which established eukaryotic cell cultures are treated with ExoS producing bacteria. To differentiate the effects of ExoS from other bacterial factors, the effects of an ExoS producing strain (388) were compared to the effects of an isogenic ExoS-deletion mutant strain $(388 \Delta S)(170)$. In co-culture analyses of epithelial cells, T3S-T-ExoS caused a general inactivation of cell function, including an inhibition of DNA synthesis, loss of re-adherence and cytoskeletal alterations (169). In other cell culture models of infection, ExoS has been implicated as a cytotoxin and an anti-phagocytic or anti-invasive factor $(38,66,192)$. The complex effects of ExoS on eukaryotic cell function are believed to relate to its bi-functional mode of action. The GAP activity of ExoS has been linked to transient cytoskeletal alterations (178) and antiphagocytic capabilities $(66,192)$, while the ADPRT activity of ExoS was found to be required for the effects of T3S-T-ExoS on human epithelial cell DNA synthesis, readherence and permanent cytoskeletal alterations (64).

T3S-T-ExoS ADPRT activity targets multiple cellular LMWG-proteins $(63,65,89$, 153). Ras was the first cellular protein to be identified as a substrate by T3S-T-ExoS ADPRT activity. ADP-ribosylation of Ras results in an alteration in protein mobility detected by SDS-PAGE and two-dimensional electrophoresis (2DE). To confirm that the alteration in mobility related to the transfer of an ADP-ribose moiety, in vitro reactions, which included purified ExoS, recombinant Ras, 14-3-3 and NAD were performed, and the alteration in Ras mobility corresponded to that caused by T3S-T-ExoS. Additionally, when cellular pools of NAD were radiolabeled, and cells were incubated with ExoS producing bacteria radiolabeled ADP-ribose was transferred to immunoprecipitated Ras. 
This study confirmed that T3S-T-ExoS ADP-ribosylates cellular Ras (153). Using a similar strategy to identify other LMWG-proteins ADP-ribosylated by ExoS, in vivo and in vitro ExoS ADPRT reactions were performed and alterations in protein mobility were compared by SDS-PAGE and 2DE. These studies led to the identification of RalA, Rab5, Rab7, Rab8, Rab11 and Rac1, but not Rab4 or Rho as cellular substrates of T3S-T-ExoS ADPRT activity (Figure 7$)(63,65)$. Cdc42, a member of the Rho family of proteins, was also ADP-ribosylated by T3S-T-ExoS, but not as efficiently as other proteins examined (65). Notably, Rab4 was not ADP-ribosylated by T3S-T-ExoS, but was ADP-ribosylated by ExoS in vitro $(30,65)$, indicating that the host cell confers T3ST-ExoS target protein specificity. More recent studies have also identified non-LMWGprotein targets of T3S-ExoS ADPRT activity including ezrin/radixin/moesin proteins, which help regulate actin cytoskeleton structure, (150), and cyclophilin A, a peptidylprolyl cis-trans isomerase (44). 


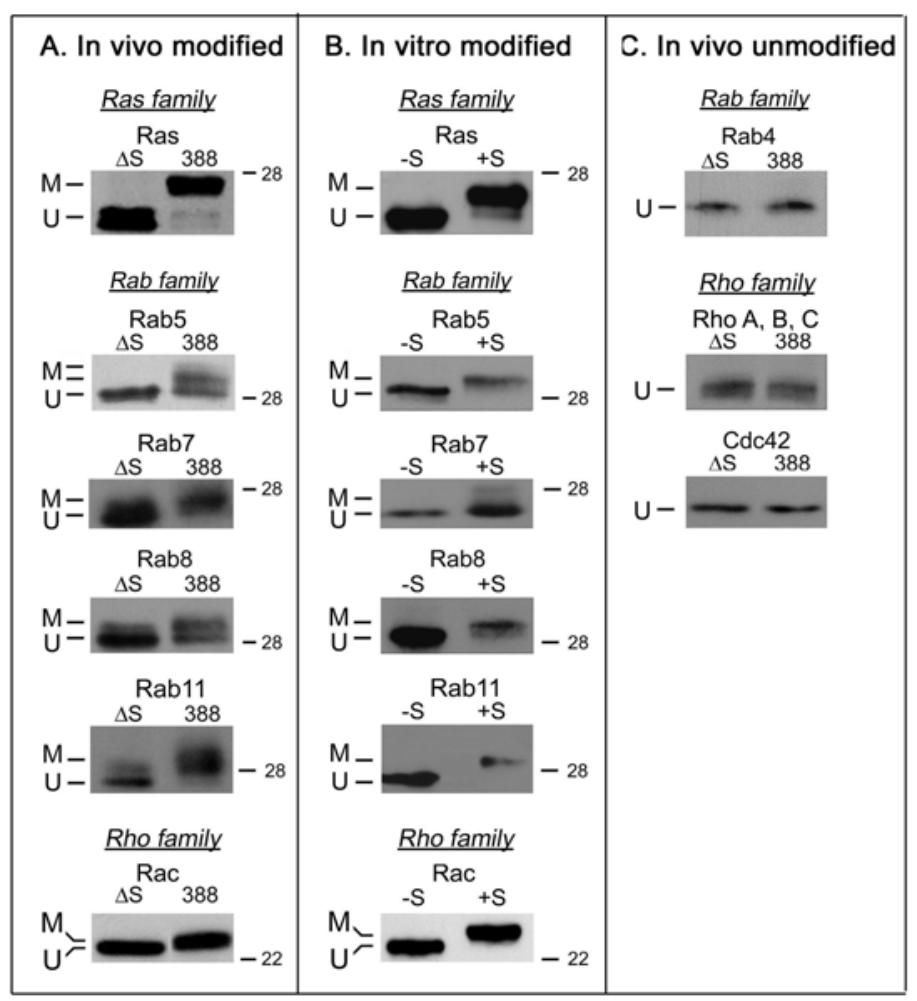

Figure 7. Proteins ADP-ribosylated by ExoS.

(A and C) In vivo analysis: HT-29 cells were grown to $50-65 \%$ confluency and cocultured with $1 \times 10^{7}$ colony forming units (cfu)/ml of 388 or $388 \Delta \mathrm{S}(\Delta \mathrm{S})$ for 4 to 5 hours. To examine Ras modification, cells were lysed and Ras was immunoprecipitated from cell lysates. To examine other proteins, eukaryotic cells were lysed in Laemmli sample buffer (129). Samples were resolved by SDS-PAGE and probed for the indicated LMWG-protein by Western blot analysis. (B) In vitro analysis: $1 \mu \mathrm{g}$ of purified protein $(\mathrm{H}-$ Ras or Rac1) or $50 \mu$ of HT-29 cell lysates (Rab proteins) were incubated without or with $0.2 \mu \mathrm{M}$ purified ExoS, $10 \mathrm{mM}$ NAD and a source of 14-3-3 protein. Reactions were terminated by the addition of $4 \mathrm{X}$ Laemmli sample buffer and heated at $95^{\circ} \mathrm{C}$ prior to resolution by SDS-PAGE and Western blot analysis. Relative molecular mass of the LMWG-proteins, and ADP-ribosylated (M) and un-ADP-ribosylated $(U)$ proteins are indicated. The results are representative of multiple independent studies.

This figure originally was originally published in Biochem. Biophys. Res. Commun (291: 91-100, 2002) in an article entitled, "Eukaryotic cell determination of ExoS ADPribosyltransferase substrate specificity" by J. E. Fraylick, E. A. Rucks, D. M. Greene, T. S. Vincent and J. C. Olson. The figure and selected text from the original figure legend appears with permission by Elsevier Science (USA). 
To determine if the cellular localization of the LMWG-proteins influences ADPribosylation by ExoS, HT-29 cells were fractionated into S100, cytosol, and P100 membrane fractions following treatment with ExoS producing bacteria. Proteins that localize to the plasma membrane fraction, like RalA and Rab5, were the first to be ADPribosylated by T3S-T-ExoS (65). These results are consistent with the notion that the MLD of ExoS targets ExoS to eukaryotic membranes where its ADPRT activity is enhanced $(176,188)$. The cellular fractionation studies revealed a different finding relative to the ADP-ribosylation of Rac1, which localizes primarily to the cytosol. While Rac1 was ADP-ribosylated in both the cytosolic and membrane fractions (193), the majority of T3S-T-ExoS ADP-ribosylated Rac1 was found in the membrane fraction (65, 193).

Since the cellular localization of LMWG-proteins appeared to influence the targeting ExoS ADPRT activity in the membrane fraction $(65,193)$. Time course studies were performed to examine trafficking patterns of ExoS ADPRT activity within HT-29 cells (Figure 8). After a 3 hour exposure to ExoS-producing bacteria, Ras and RalA, both of which localize primarily to the plasma membrane, displayed small shifts in mobility, as did Rab5, which localizes to early endosomes. By 4 hours, Rab8 and Rab11, both of which localize to recycling endosomes, were ADP-ribosylated. The last proteins to be modified by T3S-T-ExoS, following a 5 hour exposure to bacteria, were Rab7, which localizes to late endosomes, and Rac1, which is primarily a cytosolic protein that cycles to the plasma membrane when GTP-bound (65). Combined, these studies indicate that features of the host cell determine the specificity and order in which eukaryotic proteins are targeted by T3S-T-ExoS ADPRT activity. 


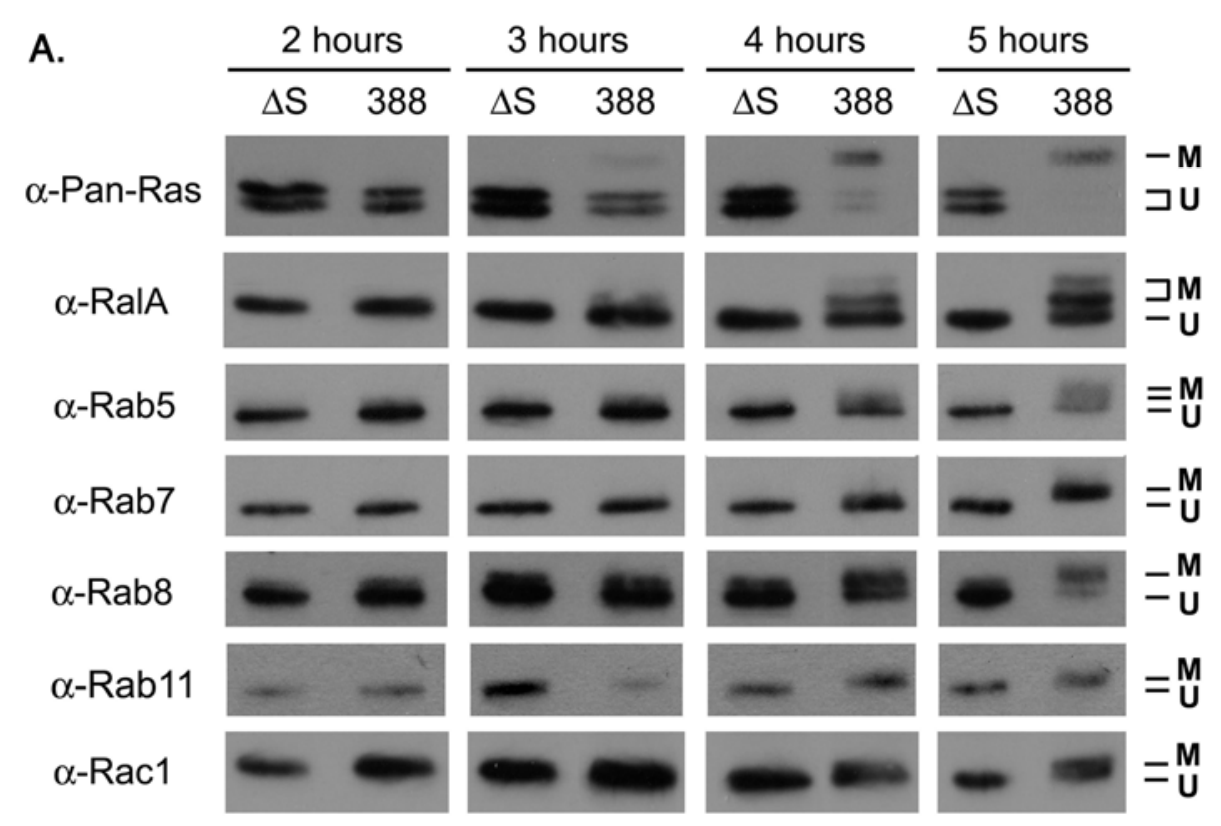

Figure 8. Time course analysis of T3S-T-ExoS substrate modification.

HT-29 cells were co-cultured with strain 388 or $388 \Delta S(\Delta S)$ for 2 to 5 hours.

Substrate modification was analyzed as described in Figure 7 . The results compare substrate modification on the same samples from one experiment, and are representative of studies performed three times. The mobilities of ADP-ribosylated proteins $(M)$ and unmodified proteins $(U)$ are indicated.

This figure originally was originally published in Biochem. Biophys. Res. Commun (291: 91-100, 2002). The title of the article is, "Eukaryotic cell determination of ExoS ADP-ribosyltransferase substrate specificity" and authors are J. E. Fraylick, E. A. Rucks, D. M. Greene, T. S. Vincent and J. C. Olson. The figure and selected text from the original figure legend appears with permission by Elsevier Science (USA). 
ExoS was found to ADP-ribosylate specific arginine residues within eukaryotic substrates (30). The arginine residues targeted are often within functional domains of the LMWG-proteins involved in guanine nucleotide binding. This is the case for Ras, RalA, and Rac1 $(63,71,193,241)$. In assessing the consequences of ExoS ADPribosylation, Ras modification was linked to the effects of ExoS on DNA synthesis. In addition, a direct correlation was observed between the severity of the effect of ExoS on DNA synthesis and the efficiency of Ras ADP-ribosylation (153). In concordance with this finding, ADP-ribosylation of Ras was found to inhibit its ability to interact with its guanine nucleotide exchange factor (GEF), which activates G-proteins by exchanging GTP for GDP. Additionally, T3S-T-ExoS ADP-ribosylation of Ras interfered with its interaction with downstream effector Raf-1, thereby interfering with activation of the MAP-kinase, Erk-2 pathway that leads to cellular proliferation $(73,92,241)$. However, mutating R41 in Ras, which prevents T3S-T-ExoS ADP-ribosylation at the site of Ras that interferes with GEF interaction, did not inhibit the effects of ExoS on DNA synthesis (J.C. Olson and T.S. Vincent, unpublished observation). These results support that ExoS ADP-ribosylation of Ras does not alone explain the effects of ExoS on DNA synthesis.

RalA is involved in signaling processes that affect cell proliferation, vesicular transport and cytoskeletal structure $(50,158,226,229)$. ADP-ribosylation of RalA by ExoS was found to inhibit interaction with its downstream effector Ral binding protein 1 (63), implicating modification of RalA in the cellular effects elicited by ExoS. Rab proteins control distinct pathways in endocytosis and vesicular trafficking that affect membrane recycling (194). In vitro analysis found the ADP-ribosylation of Rab5 by 
ExoS to interfere with its ability to bind EEA1, retarding endosome-endosome fusion (10). Thus, T3S-T-ExoS has the potential to interfere with multiple cell signaling pathways. It has been difficult for investigators to assign a individual target proteins to each phenotype caused by ExoS ADP-ribosylation since there is cross-talk between many of these pathways. However, it is likely that the effects of ExoS on proliferation, cytoskeleton and readherence mechanisms relate to the targeting of multiple eukaryotic cell substrates.

The examination of multiple human cell lines has identified differences in cellular sensitivities to T3S-T-ExoS. Normal, confluent, polarized epithelial monolayers were found to be highly resistant to the effects of T3S-T-ExoS $(58,154)$. However, when these cell monolayers were disrupted with EDTA-treatment or tested subconfluently, cells became sensitive to the effects of T3S-T-ExoS and ADP-ribosylation of Ras was observed (154). In examining the effects of ExoS producing bacteria on epithelial cell lines derived from different tissues, it was noted that tumor-derived cell lines were highly sensitive to the effects of ExoS on cell growth (Figure 9) (154). The detection of cellular differences in sensitivity to T3S-T-ExoS, along with the finding that alterations in cellular physiology can affect sensitivity to ExoS, supported the notion that eukaryotic cell factors can influence the T3S-T and ultimately the toxicity of ExoS. The purpose of the studies in this dissertation is to examine the host cell role in the T3S-T of ExoS, and subsequent cellular targeting of T3S-T-ExoS ADPRT activity. 


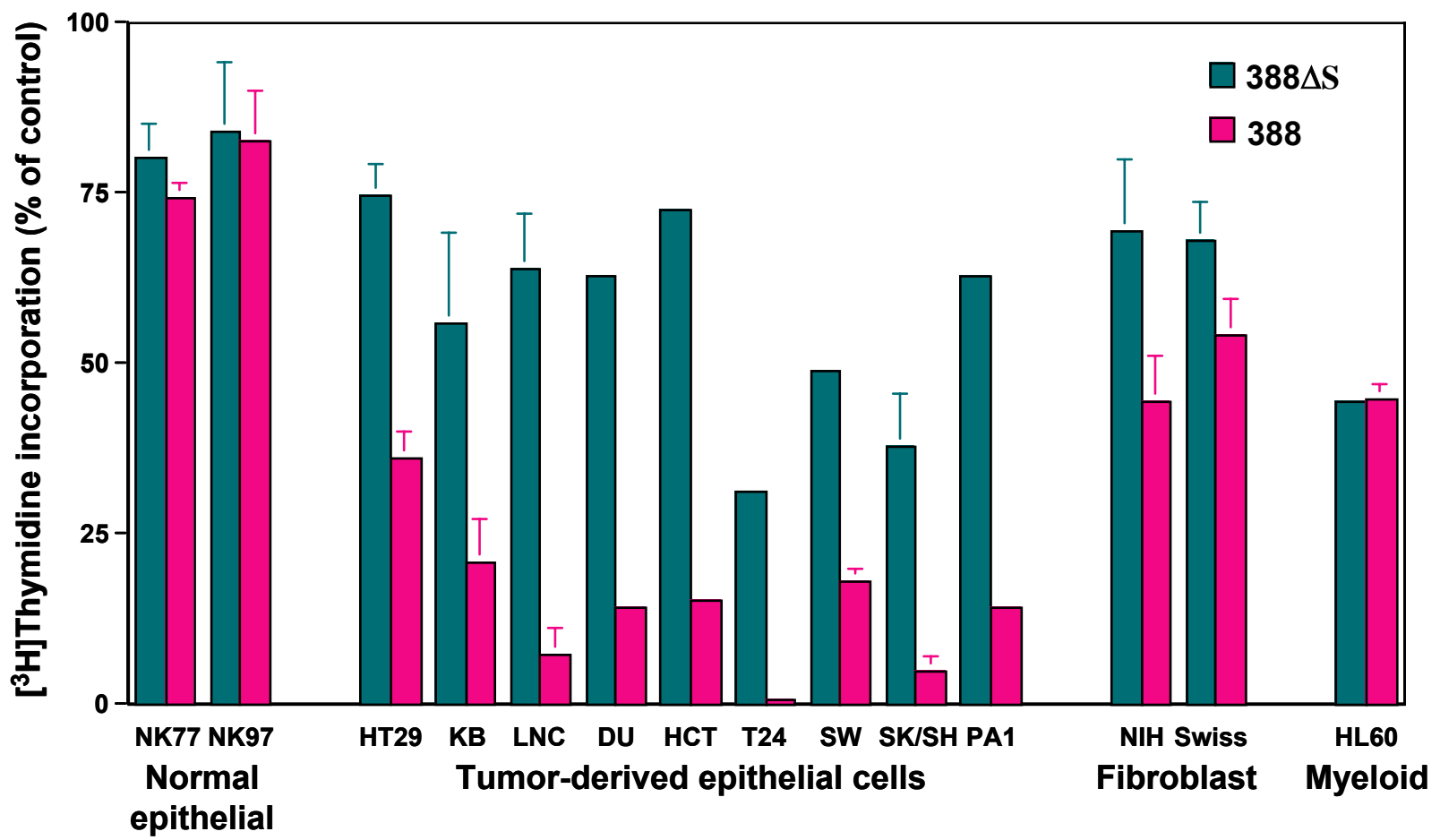

Figure 9. Differential effects of T3S-T-ExoS on DNA synthesis.

Different cell lines were assayed for the effect of T3S-T-ExoS on DNA synthesis based on $\left[{ }^{3} \mathrm{H}\right]$ Thymidine incorporation after a 4 to 5 hour co-culture with strains 388 or $388 \Delta S(170)$. The difference in inhibition of DNA synthesis between non-ExoS producing strain, $388 \Delta S$, and ExoS producing strain, 388, is attributed to an ExoS specific effect on DNA synthesis. Data shown is representative of studies performed multiple times and the mean and standard deviation of $\left[{ }^{3} \mathrm{H}\right]$ Thymidine incorporation relative to non-bacterial treated controls are shown. 
Research Objectives:

Hypothesis:

The host cell can influence the T3S-T of effector proteins at the level of membrane translocation or, once translocated, through the intracellular targeting of effector protein activity.

Specific Aim 1: To characterize eukaryotic cell differences in the function and substrate specificity of ExoS ADPRT activity when translocated by Pseudomonas T3S.

Specific Aim 2: To determine the cellular mechanism associated with phorbol esterinduction of sensitivity in T3S-ExoS-resistant HL-60 cells.

Specific Aim 3: To determine the mechanism associated with ExoS substrate specificity as it may relate to the trafficking, processing or localization of ExoS within the eukaryotic cell. 


\section{Chapter 2: Cell line differences in bacterially translocated ExoS ADP- ribosyltransferase substrate specificity}

Contents of this chapter were originally published in 2003 in Volume 149 of

Microbiology, pages 319-331 and appear with permission from the Society for General Microbiology. Authors of the published manuscript are E. A. Rucks, J. M. Fraylick, L. M. Brandt, T. S. Vincent, and J. C. Olson. 


\begin{abstract}
Exoenzyme S (ExoS) is an ADP-ribosyltransferase (ADPRT) directly translocated into eukaryotic cells by the type III secretory (T3S) process of Pseudomonas aeruginosa $(\mathrm{Pa})$. Comparisons of the effects of ExoS on human epithelial and murine fibroblastic cells found that human epithelial cells exhibited an overall increased sensitivity to the effects of bacterially translocated ExoS on cell proliferation, morphology and readherence. ExoS was also found to ADP-ribosylate a greater number of low molecular weight G- (LMWG-) proteins in human epithelial cells, as compared to murine fibroblasts. Examination of the mechanism for differences in ExoS ADPRT substrate modification found that the more restricted pattern of substrate modification in murine fibroblasts was not linked to the efficiency of bacterial adherence or to the efficiency of ExoS internalization by the T3S process. In exploring the nature of patterns of substrate modification, more extensive substrate modification was detected in human and simian cell lines, while rodent cell lines, including rat, mouse and hamster, consistently exhibited the more limited pattern of LMWG-protein ADP-ribosylation. Patterns of substrate modification were not altered by transformation and occurred independently of cell type. These studies suggest that properties related to the animal origin of eukaryotic cells affect the substrate targeting of ExoS ADPRT activity, and that this in turn can influence the severity of effects of ExoS on host cell function.
\end{abstract}




\section{Introduction}

$P a$ is a ubiquitous environmental organism that can thrive in a variety of environmental niches, including soil, rivers, and biofilms. If it is allowed to transverse normal physical or immunological barriers, $P a$ can adapt to become a highly virulent opportunistic pathogen. The success of $\mathrm{Pa}$ as an opportunistic pathogen relates to its ability to produce a large number of virulence factors and to its high antibiotic resistance. One virulence factor produced by $\mathrm{Pa}$, ExoS, is an ADPRT that is directly translocated into eukaryotic cells by the T3S process (258). Although the precise role of ExoS in Pa virulence is not fully understood, the exoS gene is found in a majority of clinical isolates (52), and there is a 6-fold greater risk of mortality associated with the expression of T3S effectors, including ExoS, in Pa lower respiratory and systemic infections (196). In cell culture models of infection, ExoS has been implicated as an antiphagocytic or anti-invasive factor $(38,66)$. In analyses of epithelial cells, bacterial translocation (T3S-T) of ExoS has been associated with a general inactivation of cell function, including an inhibition of DNA synthesis, loss of re-adherence and cytoskeletal alterations (169).

The complex effects of ExoS on eukaryotic cell function are believed to relate to its bi-functional mode of action. ExoS includes a GTPase activating (GAP) function within its amino-terminus (77), and an ADPRT activity within its carboxy-terminus (117). The GAP activity of ExoS has been linked to cytoskeletal alterations (178), while the ADPRT activity of ExoS is required for the effects of T3S-T-ExoS on human epithelial cell DNA synthesis, re-adherence and long-term cytoskeletal alterations (64). When translocated by T3S, ExoS ADPRT activity targets multiple cellular LMWG-proteins $(63,65,153)$ and 
requires a eukaryotic cofactor, 14-3-3, for catalytic activity $(29,68,90)$. ADP-ribosylation of cellular targets, Ras and RalA, has been found to interfere with their respective signal transduction pathways $(63,73,91,241)$, thereby linking a cellular mechanism to the effects of ExoS on eukaryotic cell function. Recent studies have drawn attention to possible modulatory influences the eukaryotic cell can have on ExoS function. Notably, ExoS ADPRT activity was found to be enhanced in the membrane compartment of epithelial cells when T3S-T $(64,188)$. In addition, an E381A mutant form of ExoS, which is unable to ADP-ribosylate Ras in vitro, was found to modify Ras when translocated into cells by the T3S process.

The examination of multiple human epithelial cell lines has identified differences in cellular sensitivities to T3S-T-ExoS. Normal, confluent, polarized epithelial monolayers were found to be highly resistant to the effects of T3S-T-ExoS $(58,154)$. However, when normal epithelial cell monolayers were disrupted or subconfluent, cells became sensitive to ExoS (154). In examining the effects of ExoS producing bacteria on epithelial cell lines derived from different tissues, it was noted that tumor-derived cell lines were highly sensitive to the effects of ExoS on cell growth (154). The detection of cellular differences in sensitivity to T3S-T-ExoS, along with the finding that alterations in cellular physiology can affect sensitivity to ExoS, support the notion that eukaryotic cell factors can influence the toxicity of ExoS.

To clarify how the eukaryotic cell might contribute to the toxicity of T3S-T-ExoS, we examined how different cell lines compared in their response to ExoS producing bacteria. Our studies found murine fibroblasts to be more resistant to the effects of ExoS than human epithelial cells. The increased resistance of murine fibroblastic cells 
to ExoS was associated with less efficient ADP-ribosylation of cellular substrates by ExoS, and occurred independently of detectable differences in the efficiency of T3S-T of ExoS. In examination of a rodent, simian, and human cell lines, distinct patterns of ExoS ADPRT substrate modification were detected, with human cell lines showing both more efficient and more extensive LMWG-protein substrate modification. These studies support that once internalized, host cell properties can influence the substrate specificity of ExoS ADPRT activity, which corresponds with alterations in the severity of effects of ExoS on cell function.

\section{Methods and Materials}

Bacterial strains and culture conditions. $P a$ strains used in this study include the prototype ExoS producing strain 388 (106) and its isogenic mutant, strain $388 \Delta$ exoS, which lacks ExoS production due to an allelic exchange of the majority of the exoS structural gene for a tetracycline resistance gene cartridge (126). Complementation studies have confirmed that differences in the effects of strains 388 and $388 \Delta \mathrm{S}$ on eukaryotic cell function relate to the production of ExoS (169). To further rule out the role of ExoT in our analyses (which is expressed by strains 388 and $388 \Delta \mathrm{S}$ ) a $388 \Delta$ ExoT mutant (256) was included in all experiments as a control. All bacterial strains used in this study were kindly provided by Dara Frank from the Medical College of Wisconsin, Milwaukee, WI. Bacteria were grown and prepared for co-culture studies with eukaryotic cells as previously described $(153,170)$.

Eukaryotic cell culture. CHO-K1, Cos-1, Detroit 532, HT-1080, HT-29, LNCaP, NIH3T3, Swiss-3T3, TCMK-1, T24 and Vero cells used in this study were obtained from the American Type Culture Collection (ATCC; Rockville, MD). RHEC cells were provided by 
Carwile LeRoy, and WI-38 and WI-38 SV40 transformed cells were kindly provided by Daohong Zhou, both from the Medical University of South Carolina, Charleston, SC. Characteristics of cell lines used in this study are shown in Table 1. Cell lines were cultured, as described below, in their respective medium containing $10 \% \mathrm{v} / \mathrm{v}$ fetal bovine serum (FBS) and $100 \mathrm{units} / \mathrm{ml}$ penicillin and $100 \mu \mathrm{g} / \mathrm{ml}$ streptomycin (PS) at $37^{\circ} \mathrm{C}$ in $5 \%-$ $\mathrm{CO}_{2}-95 \%$ air. All cell culture media and components were purchased from Gibco-BRL (Gaithersburg, MD).

Epithelial cell lines. CHO-K1 cells (ATCC CCL 61) were cultured in Ham's F12K medium and split 1:6 at confluency and passaged. HT-1080 (ATCC CCL-121), RHEC, TCMK-1 (ATCC CCL 139), and Vero cells (ATCC CCL 81) were cultured in Dulbecco's Modified Eagle's Medium (DMEM), split 1:6-1:10 at confluency and passaged. HT-29 cells (ATCC HTB 38) were maintained in McCoy's 5A medium, as previously described (169). LNCaP cells (ATCC CRL 1740) were cultured in RPMI medium and split 1:4 at confluency and passaged. T24 cells (ATCC HTB 4) were grown in McCoy's 5A medium, split 1:6 at confluency and passaged twice weekly. 


\begin{tabular}{|c|c|c|c|c|c|c|c|c|}
\hline \multirow[t]{2}{*}{ Cell line } & \multirow[t]{2}{*}{ Source } & \multirow[t]{2}{*}{ Cell type* } & \multirow[t]{2}{*}{ Immortal } & \multirow[t]{2}{*}{ Tumorigenic } & \multicolumn{4}{|c|}{ Substrates $\dagger$} \\
\hline & & & & & RalA & Rac1 & Rab5 & Rab8 \\
\hline $\mathrm{CHO}-\mathrm{K} 1$ & Hamster & Epithelial (ovary) & Yes & No & + & - & - & - \\
\hline RHEC & Rat & Endothelial (heart) & Yes $\ddagger$ & No & + & - & - & - \\
\hline TCMK-1 & Murine & Epithelial (kidney) & Yes $\ddagger$ & No & + & - & - & - \\
\hline NIH-3T3 & Murine & Fibroblast (embryo) & Yes & No & + & - & - & - \\
\hline Swiss-3T3 & Murine & Fibroblast (embryo) & Yes & No & + & - & - & - \\
\hline Cos-1 & Simian & Fibroblast (kidney) & Yes $\ddagger$ & No & ++ & \pm & + & + \\
\hline Vero & Simian & Epithelial (normal kidney) & Yes & No & ++ & \pm & + & + \\
\hline HT- 1080 & Human & Epithelial (fibrosarcoma) & Yes & Yes & ++ & + & + & + \\
\hline HT-29 & Human & Epithelial (colon) & Yes & Yes & ++ & + & + & + \\
\hline LNCaP & Human & Epithelial (prostate) & Yes & Yes & ++ & + & + & + \\
\hline T24 & Human & Epithelial (bladder) & Yes & Yes & ++ & + & + & + \\
\hline WI-38 & Human & Fibroblast (normal lung) & No & No & ++ & + & + & + \\
\hline WI-38t† & Human & Fibroblast (lung) & Yes $\ddagger$ & No & ++ & + & + & + \\
\hline Detroit 532 & Human & Fibroblast (foreskin) & Yes & No & + & - & - & - \\
\hline
\end{tabular}

Table 1. LMWG-protein substrate modification by T3S-T-ExoS in different cell lines.

${ }^{*}$ Cell type, immortality and tumorgenicity as indicated by the ATCC, except for the RHEC cell line, which was obtained from Carwile LeRoy. $\uparrow+$, ADP-ribosylation by ExoS; ++, two ExoS ADP-ribosyl-modified isoforms; -, not ADP-ribosylated by ExoS. $\ddagger$ SV-40 transformed.

Fibroblastic cell lines. Detroit 532 cells (ATCC CCL 54) were grown in RPMI medium and maintained as previously described (170). Cos-1 cells (ATCC CRL 1650), NIH-3T3 cells (ATCC CRL 1658), Swiss-3T3 cells (ATCC CCL 92), WI-38 and WI-38 SV40 transformed (WI-38t) were cultured in DMEM and split 1:6 and passaged twice a week. Co-culture of eukaryotic cells with bacteria. For bacterial/eukaryotic cell co-culture studies, eukaryotic cells were detached with $0.05 \% \mathrm{w} / \mathrm{v}$ trypsin-0.53 mM EDTA (trypsinEDTA) and seeded at $1 \times 10^{5}$ cells $/ \mathrm{ml}$ in 6,24 or 48 -well plates or culture dishes (Costar; Cambridge, MA). Cells were grown for either 24 hours or 48 hours (depending on the growth rate of the cell line) to obtain $60-80 \%$ confluency prior to co-culture with 
bacteria. Strain 388 or $388 \Delta S$ bacteria were diluted, based on culture $\mathrm{OD}_{590}$, to $1 \times 10^{7}$ $\mathrm{cfu} / \mathrm{ml}$ in medium specific for each eukaryotic cell line containing $0.6 \% \mathrm{w} / \mathrm{v}$ bovine serum albumin (BSA). A multiplicity of infection (MOI). range of 10 to 100 was used in these studies, unless otherwise indicated, and bacteria were co-cultured with eukaryotic cells for 2 to 6 hours. Control cells received the respective medium with no bacteria. Effects of bacteria on eukaryotic cell function were analyzed as described below.

Quantification of DNA synthesis. Following a 4 hour co-culture period, bacteria were removed and cells were washed with culture medium containing $10 \% \mathrm{FBS}, 200 \mu \mathrm{g} / \mathrm{ml}$ gentamicin and $100 \mu \mathrm{g} / \mathrm{ml}$ ciprofloxacin (GC-medium) to inhibit further bacterial growth. Cells were pulsed for 20 hours in GC-medium containing $1 \mu \mathrm{Ci} / \mathrm{ml}$ [methyl-3H]thymidine (25 Ci mmol-1; Amersham, Arlington Heights, IL). DNA synthesis was quantified as previously described (170) and is reported as the percent of [3H]thymidine incorporated relative to that of non-bacterial treated control cells.

Analysis of cell viability. Cell viability was examined following a 3, 4.5 and 6 hour coculture period, after which bacteria were removed, and total cell viability was determined by trypan blue exclusion immediately after removal of bacteria and following an extended 20 hour culture period in GC medium.

Examination of cell morphology. To compare long-term effects of ExoS on cellular morphology, cells were co-cultured with bacteria for 4 hours, bacteria were removed, cells were washed with PBS and cultured in GC-medium for an additional 20 hours. Morphological alterations were examined by phase contrast microscopy. Analysis of eukaryotic cell adherence. The effects of ExoS producing bacteria on eukaryotic cell adherence to matrix were assessed after a 2 to 6 hour co-culture period 
by quantifying adherent and non-adherent cells, using trypan blue exclusion, 20 or 40 hours after bacteria were removed. To assess the effects of ExoS on re-adherence, cells were detached with trypsin-EDTA following co-culture with bacteria, washed, resuspended in GC-medium, re-plated and allowed to re-adhere for 20 hours (HT-29, NIH-3T3, Swiss-3T3 cells) or 48 hours (LNCaP cells). (LNCaP cells were previously recognized to require a longer adherence time (98)). The number and viability of nonadherent and trypsinized, adherent cells were determined after this time based on trypan blue exclusion. The percentage of viable and non-viable, adherent or nonadherent cells was calculated relative to total cell number.

Bacterial association with eukaryotic cells. To compare the adherence of bacteria to different eukaryotic cell lines, cells were seeded in 24-well plates, as described above, and co-cultured with bacteria at a MOI of approximately 200:1 for 4.5 hours (HT-29, $\mathrm{NIH}-3 \mathrm{~T} 3$, and Swiss-3T3 cells) or 3 hours (LNCaP cells). (The limited time of exposure of $\mathrm{LNCaP}$ cells related to their increased sensitivity to bacterial effects on cell viability.) Bacteria were removed, and $1 \mathrm{ml}$ HEPES buffered saline (HBS) $(137 \mathrm{mM} \mathrm{NaCl}, 4 \mathrm{mM}$ $\mathrm{KCl}, 10 \mathrm{mM}$ Hepes $\mathrm{pH}$ 7.4, $11 \mathrm{mM}$ glucose) was added to wells. Cells were detached by scraping, transferred to microcentrifuge tubes, and washed three times with HBS, centrifuging cells at $400 \mathrm{~g}$ for 4 minutes at $4^{\circ} \mathrm{C}$ between washes. Eukaryotic cells were lysed in PBS containing $0.25 \% \mathrm{v} / \mathrm{v}$ Triton $\mathrm{X}-100$ for 30 minutes on ice, and lysates were diluted 1:1000 and plated on Luria broth (LB) agar plates. Bacterial colonies were counted 16 hours later, and the number of bacteria associated per eukaryotic cell was determined. Data were analyzed for statistical significance using one-way factorial ANOVA (http://members.aol.com/johnp71/anova1sm.html). 


\section{ADP-ribosylation of cellular substrates by bacterially translocated ExoS.}

In vivo. In vivo ADP-ribosylation of LMWG-protein substrates by bacterially translocated ExoS was assessed based on altered protein mobility by SDS-PAGE following coculture with bacteria for 4 or 6 hours, as previously described $(64,65,153)$. To examine Ras modification, cells were lysed in TBS-TDS (10 mM Tris, pH 7.4, $140 \mathrm{mM} \mathrm{NaCl}, 1 \%$ $\mathrm{v} / \mathrm{v}$ Triton X-100, $0.5 \% \mathrm{w} / \mathrm{v}$ sodium deoxycholate, $0.1 \% \mathrm{w} / \mathrm{v}$ SDS), and cellular Ras was immunoprecipitated using Y13-259 anti-pan Ras antibody, as previously described (153). Precipitates were resolved by SDS-12.5\% PAGE (129), transferred to PVDF membranes (Millipore; Bedford, MA) (233), and Ras was detected using $100 \mathrm{ng} / \mathrm{ml}$ LA045 pan-Ras antibody or 100 ng/ml LA069 H-Ras specific antibody (Quality Biotech; Camden, NJ), followed by horseradish peroxidase (HRP)-conjugated anti-mouse IgG (Jackson Immuno Research; West Grove, PA) and visualized by enhanced chemiluminescence (ECL) (Amersham). RalA, Rab5, Rab7, Rab8, Rab11 and Rac1 modification was detected following lysis of cells with Laemmli electrophoresis sample buffer, and lysates were resolved by SDS-12\% PAGE and transferred to PVDF membranes, as described above. Individual LMWG-proteins were detected using the following antibodies: RalA, $50 \mathrm{ng} / \mathrm{ml}$ mouse anti-RalA monoclonal antibody (Transduction Laboratories; Lexington, Kentucky); Rab5, $10 \mathrm{ng} / \mathrm{ml}$ rabbit anti-Rab5B (A20) polyclonal antibody (Santa Cruz Biotechnology; Santa Cruz, California); Rab7, 0.7 $\mu \mathrm{g} / \mathrm{ml}$ goat anit-Rab7 (C-19) polyclonal antibody (Santa Cruz); Rab8, $250 \mathrm{ng} / \mathrm{ml}$ mouse anti-Rab8 monoclonal antibody (Transduction Laboratories); Rab11, $250 \mathrm{ng} / \mathrm{ml}$ mouse anti-Rab11 monoclonal antibody (Transduction Laboratories); and Rac1, $250 \mathrm{ng} / \mathrm{ml}$ mouse anti-Rac1 monoclonal antibody (Transduction Laboratories). Immunoblots were 
developed with HRP-conjugated anti-mouse IgG (Sigma) or anti-rabbit IgG (Transduction Laboratories), as appropriate, and visualized by ECL.

In vitro. In vitro ADP-ribosylation of fibroblastic RalA, Rab proteins, or Rac1 was performed, as previously described (65), using $0.2 \mu \mathrm{M}$ purified ExoS (153), $10 \mathrm{mM}$ NAD (Sigma) and $40 \mu \mathrm{l}$ of $\mathrm{NIH}-3 \mathrm{~T} 3$ or Swiss-3T3 fibroblast cell lysate (as a source of the endogenous proteins and 14-3-3 cofactor) in a $100 \mu \mathrm{l}$ reaction volume, which was incubated for 30 minutes at room temperature. Reactions were stopped with $4 \mathrm{x}$ Laemmli sample buffer and heating at $95^{\circ} \mathrm{C}$ for 5 minutes. Fibroblast cell lysates were prepared by incubating cells on ice for 30 minutes in lysis buffer containing $50 \mathrm{mM}$ Tris, $0.1 \%$ Triton $\mathrm{X}-100,10 \mu \mathrm{g} / \mathrm{ml}$ aprotinin, $10 \mu \mathrm{g} / \mathrm{ml}$ leupeptin, $1 \mathrm{mM}$ EDTA and $100 \mu \mathrm{M}$ PMSF. Lysates were cleared of nuclei and unbroken cells by centrifugation at $16,000 x$ $\mathrm{g}$ for 5 minutes prior to use in reactions. Following the ADPRT reactions, proteins were resolved by SDS-12\% PAGE and immunoblotted for the specific proteins, as described above.

Time course analysis of ExoS internalization. Substrate modification and the internalization of ExoS ADPRT activity was assessed in fibroblastic and epithelial cells seeded in $100 \mathrm{~mm}$ dishes and co-cultured with bacteria for $3,4.5$ or 6 hours. Following removal of bacteria, cell monolayers were washed twice with PBS, and adherent cells were detached with trypsin-EDTA. Cells were recovered from washes by centrifugation at $400 \mathrm{~g}$ for $10 \mathrm{~min}$ and combined with trypsin-detached cells. The treatment of cells with trypsin in these studies minimized the likelihood that extracellularly bound ExoS would remain in cellular fractions. The cell pellet was resuspended in $100 \mu \mathrm{l}$ of cell permeabilization solution $(0.2 \% \mathrm{w} / \mathrm{v}$ saponin, $100 \mathrm{mM} \mathrm{NaCl}, 250 \mathrm{mM}$ sucrose, $5 \mathrm{mM}$ 
EDTA, $1 \mathrm{mM}$ PMSF, $10 \mu \mathrm{g} / \mathrm{ml}$, each, leupeptin and aprotinin (Sigma) and $100 \mu \mathrm{g} / \mathrm{ml}$ ciprofloxacin) and lysed on ice for 30 minutes. Lysates were centrifuged at $16,000 \mathrm{~g}$ for 20 minutes at $4^{\circ} \mathrm{C}$. The supernatant (cytosolic fraction) was removed, and the pellet was resuspended in $100 \mu \mathrm{l}$ of cell permeabilization solution containing $1 \% \mathrm{v} / \mathrm{v}$ Triton X-100 and vortexed for 15 seconds every 5 minutes four times to solubilize membrane proteins. The solubolized pellets were centrifuged at $16,000 \mathrm{~g}$ for 10 minutes, and the supernatant (membrane fraction) was removed. To analyze substrate modification in the cytosolic and membrane fractions, a $50 \mu \mathrm{l}$ aliquot of each sample was mixed with $4 \mathrm{x}$ Laemmli sample buffer, and an equivalent volume of each fraction was resolved by SDS-PAGE and immunoblotted for RalA, Rab proteins, or Rac1, as described above. ExoS ADPRT activity in remaining portions of cytosolic and membrane fractions was quantified, as previously described (54), and related to total protein concentrations in the respective sample determined using the Sigma Diagnostics Lowry-based protein assay (Sigma). Data were analyzed for statistical significance using one-way factorial ANOVA, as described above. 


\section{Results}

Comparison of effects of ExoS on cell function. We previously found that effects of T3S-T-ExoS on eukaryotic cell function could be assessed in a bacterial/eukaryotic cell co-culture model of infection by comparing alterations caused by ExoS producing strain 388 to that of the isogenic, non-ExoS producing strain $388 \Delta S(169,170)$. In comparisons of different cell lines for the effects of T3S-T-ExoS, we recognized a general increase in sensitivity of human epithelial cells to ExoS as compared to NIH3T3 and Swiss-3T3 fibroblastic cells. This difference in cell sensitivity to ExoS was consistent with previous studies which found Detroit 532 fibroblasts to be sensitive to effects of ExoS on DNA synthesis, but relatively resistant to effects of ExoS on cell morphology (170). To gain a better understanding of cellular differences in the effects of ExoS and the mechanisms associated with these differences, HT-29 and LNCaP human epithelial cells and NIH-3T3 and Swiss-3T3 fibroblastic cells were used as model cell lines to further explore differences in the effects of ExoS on cell function.

In examining effects of T3S-T-ExoS on DNA synthesis, HT-29 and LNCaP epithelial cells were found to be more sensitive to effects of ExoS on DNA synthesis, showing a $51.6 \% \pm 0.5 \%$ and $88.6 \% \pm 1.0 \%$ inhibition of DNA synthesis, respectively, relative to that of strain $388 \Delta$ S. This compared to a $36.0 \% \pm 1.3 \%$ and $20.3 \% \pm 0.7 \%$ inhibition of DNA synthesis caused by strain 388 in NIH-3T3 and Swiss-3T3 murine fibroblasts, respectively, relative to strain $388 \Delta S$. In determining how effects of ExoS on cell viability related to effects on DNA synthesis, cell viability was assayed, based on trypan blue exclusion, under the same co-culture conditions and at the same time point as DNA synthesis analyses. In these studies, LNCaP and NIH-3T3 cells exhibited a greater loss 
of viability ( $95.2 \% \pm 0.4 \%$ and $44.6 \% \pm 0.9 \%$, respectively). This compared to a minimal $6.1 \% \pm 0.9 \%$ and $6.8 \% \pm 0.2 \%$ loss of viability caused by bacterially translocated ExoS in HT-29 and Swiss-3T3 cells, respectively. These studies found that human epithelial cells were more sensitive than murine fibroblasts to effects of ExoS on DNA synthesis, and that the effects on DNA synthesis did not necessarily correlate with effects of ExoS on cell viability.

Previous studies from our laboratory found permanent, long-term effects of strain 388 on HT-29 epithelial cell rounding to be directly associated with ExoS ADPRT activity $(64,169)$. This differed from transient effects on cell morphology caused by the non-ExoS producing strain $388 \Delta S$, which were less severe and reversible upon removal of bacteria. In comparing the effects of ExoS on the morphology of these four different cell lines, severe cell rounding persisted in HT-29 and LNCaP epithelial cells 20 hours after exposure to strain 388 , while long-term rounding was limited in $\mathrm{NIH}-3 \mathrm{~T} 3$ cells and absent from Swiss-3T3 fibroblastic cells (Figure 10). 


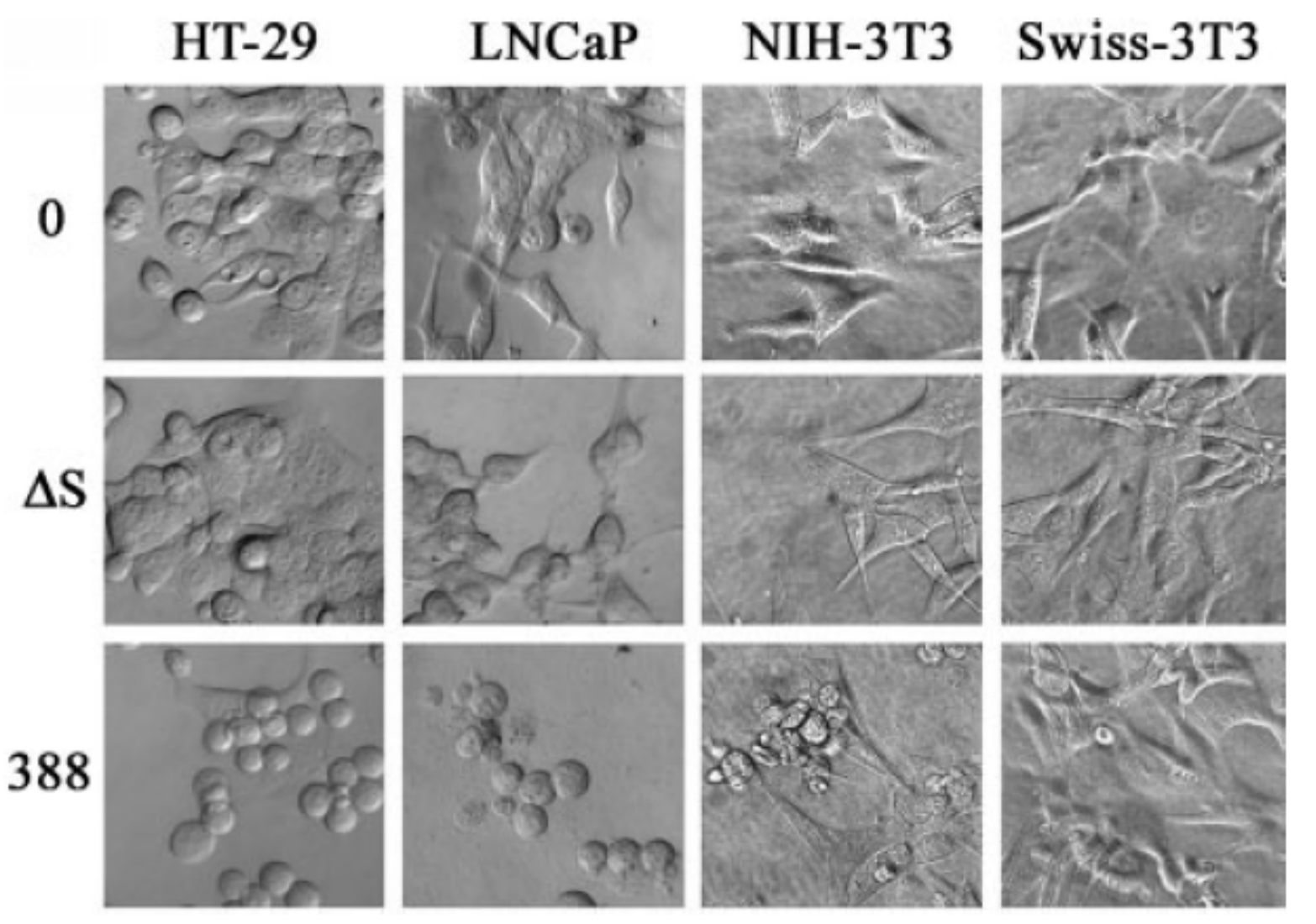

Figure 10. Effects of T3S-T-ExoS on epithelial and fibroblastic cell morphology.

HT-29, LNCaP, NIH-3T3 and Swiss-3T3 cells were seeded at $1 \times 10^{5}$ cells $/ \mathrm{ml}$ in 48-well plates, either 24 hours (fibroblasts) or 48 hours (epithelial) prior to the addition medium containing no bacteria $(0)$, or $10^{7} \mathrm{cfu} / \mathrm{ml}$ of strain 388 or $388 \Delta S(\Delta S)$ for 4 hours.

Bacteria were removed, cells were washed with PBS and cultured in GC-medium for an additional 20 hours. Long-term effects on cell morphology were examined by phase contrast microscopy at 20 hours, and images are representative of a minimum of three independent experiments. 
The functional detachment and reattachment of epithelial and fibroblastic cells to extracellular matrix is required for cell mobility and the maintenance of cellular barriers. This function was previously found to be affected in HT-29 cells following treatment with ExoS-expressing bacteria (169). In assessing whether differences exist in the sensitivity of the four cell lines to the effects of ExoS on cellular adherence and re-adherence, it was first noted that minimal direct loss of adherence occurred following the co-culture of HT-29, NIH-3T3 and Swiss-3T3 cells with ExoS-producing bacteria for 4 to 5 hours. In comparison, LNCaP cells were found to detach from the culture plate or become loosely adherent within a 4 hour exposure to bacteria (data not shown). Effects of ExoS on the reattachment processes were examined by detaching cells from plates with trypsin, following exposure to bacteria, replating, and allowing cells to re-adhere for 20 or 48 hours. In these analyses, HT-29 cells began to lose the ability re-establish adherence to matrix after a 2 to 3 hour exposure to strain 388 (Figure 11). LNCaP cells, which were sensitive to direct effects of ExoS on cell adherence, displayed a generalized sensitivity to bacterial effects on re-adherence, independently of ExoS production. NIH-3T3 cells required a greater than 4 hour exposure to strain 388 before re-attachment processes were lost, and Swiss-3T3 cells tolerated a greater than 6 hour exposure to strain 388 before loss of re-adherence. These studies found that bacterially translocated ExoS interfered with re-adherence processes differently in each cell line, but that overall, murine fibroblasts appeared more resistant to effects of ExoS on adherence processes. 


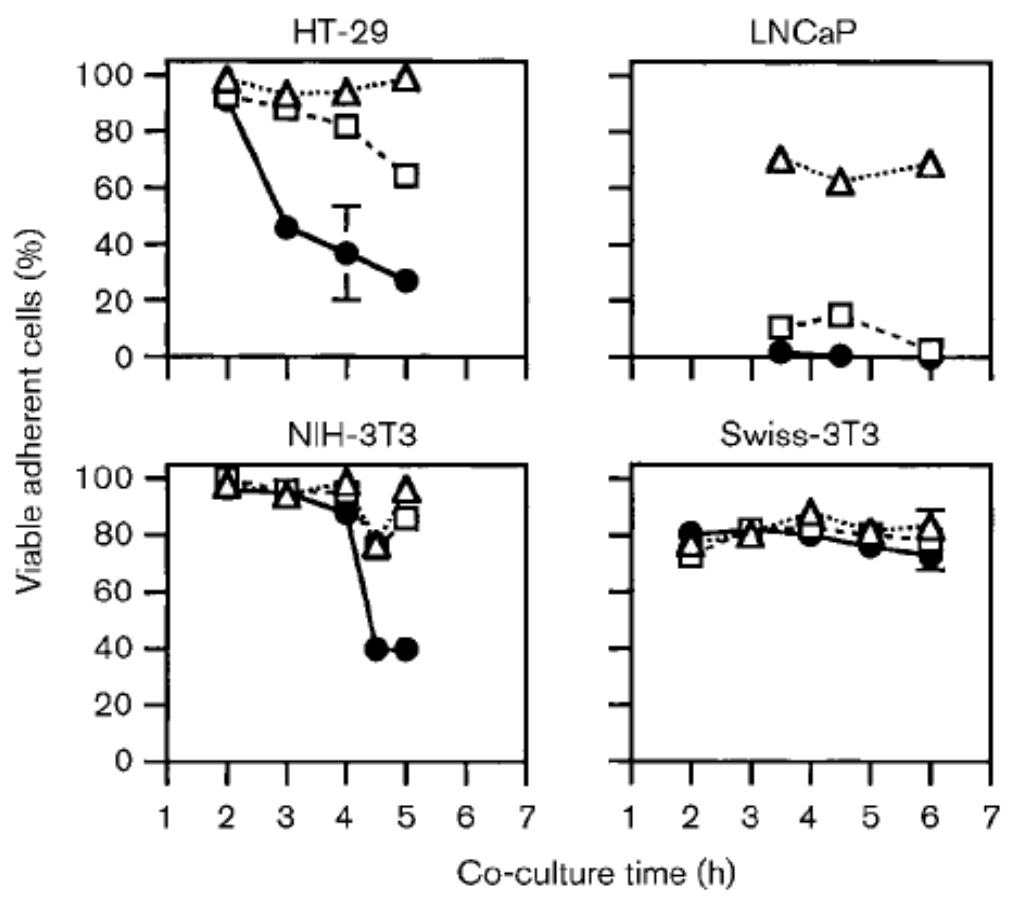

Figure 11. Effects of T3S-T-ExoS on epithelial and fibroblastic cell re-adherence.

HT-29, LNCaP, NIH-3T3 and Swiss-3T3 cells were seeded and cultured with no bacteria (open triangle) or strain 388 (filled square) or $388 \Delta S$ (open square) for 2 to 6 hours, as in Figure 1. Bacteria were removed, cells were washed, detached with trypsinEDTA, replated, and allowed to re-adhere for 20 hours (HT-29, NIH-3T3, Swiss-3T3) or 44 hours (LNCaP). The number and viability of non-adherent and trypsinized adherent cells were determined after this time based on trypan blue exclusion. The percentage of viable, adherent cells was calculated relative to total cell number. Results are expressed as percent viable adherent cells following the indicated co-culture time. The mean and SE of three to four independent experiments are represented. 
In summarizing comparisons of the effects of T3S-T-ExoS on these four cell lines, it was found that the two human epithelial cells examined were, in general, more sensitive than the murine fibroblastic cell lines to effects of ExoS on DNA synthesis, alterations in morphology, and effects on re-adherence processes. However, individual cell line variations in the effects of ExoS were also evident in these studies, highlighting the ability of eukaryotic cells to modulate, or contribute to, the effects of ExoS on cell function in a cell line-dependent manner.

Comparison of bacterial association to cellular sensitivity to ExoS. Contact between bacteria and eukaryotic cells is required for the T3S-T of effector proteins (102). To assess whether the differential sensitivity among the cell lines to ExoS might relate to efficiency of bacterial adherence, bacterial association with the different cell lines was quantified after a 3 or 4.5 hour exposure to bacteria. Bacterial adherence to LNCaP cells was assayed after a shorter, 3 hour period since this cell line begins to loose viability after a 4 hour co-culture period (refer to previous section). As shown in Figure 12, an average of 2 bacteria associated with each HT-29 epithelial cell, with 5 to 6 bacteria associating with each LNCaP cell. In analysis of the NIH-3T3 and Swiss-3T3 fibroblastic cells, an average of 20 bacteria were associated per cell, which was significantly greater $(P<0.006)$ than the number adhering to epithelial cells. Comparisons of the size of epithelial and fibroblastic cells as cultured monolayers, found fibroblasts to have about a 2.5 times greater surface area than epithelial cells, making the association of bacteria per surface area still slightly greater for fibroblastic than epithelial cells. Based on these analyses, and consistent with previous reports (58, 154), we were unable to relate the efficiency of bacterial association with cells to 


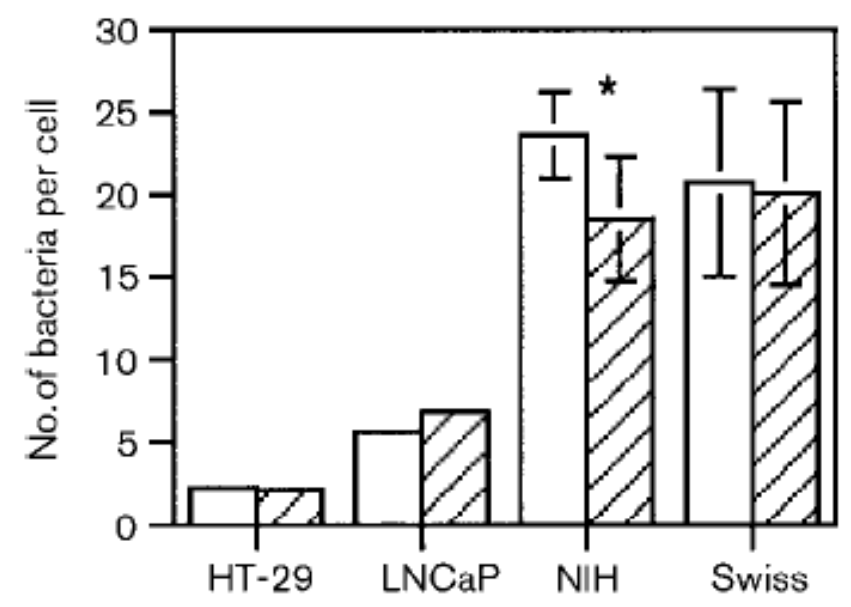

Figure 12. Bacterial association with HT-29, LNCaP, NIH-3T3 (NIH) and Swiss-3T3 (Swiss) cells.

Mammalian cells were seeded in 24-well plates as in Figure 1, and cultured with 388 (hatched bars) or 388 $\triangle \mathrm{S}$ (open bars) bacteria (MOI, 200:1) for either 3 hours (LNCaP) or 4.5 hours (HT-29, NIH-3T3 (NIH), Swiss-3T3 (Swiss)). A higher MOI. was used in these studies to maximize bacterial association. Bacteria were removed, eukaryotic cells were detached by scraping into HEPES buffered saline (HBS) and washed three times in HBS. Eukaryotic cells were lysed in PBS containing $0.25 \% \mathrm{v} / \mathrm{v}$ Triton X-100, and the lysate was diluted and plated on LB agar plates. Bacterial colonies were counted after 16 hours. Assays were performed in quintuplicate, and the number of associated bacteria per eukaryotic cells was calculated. The mean and SE of three to four independent experiments are represented. A statistically significant difference was detected between the association of strain 388 and strain $388 \Delta S$ with epithelial and fibroblastic cells, based on ANOVA; ${ }^{*}, P=0.307$ in the comparison of adherence of $388 \Delta \mathrm{S}$ and 388 to $\mathrm{NIH}-3 \mathrm{~T} 3$ cells, indicating that the differences noted were not statistically significant. 
differences in cellular sensitivity to the effects of ExoS. Also evident in Figure 12, is the lack of significant difference in the association of strain 388 or $388 \Delta S$ bacteria with the different cell lines, indicating no influence of ExoS production on bacterial adherence in these studies.

ADP-ribosylation of cellular substrates by T3S-T-ExoS. Previous studies confirmed the requirement of ExoS ADPRT activity for effects of T3S-T-ExoS on epithelial cell DNA synthesis and morphology (64). To examine how differences in the sensitivity of cell lines to ExoS might relate to cellular targets of ExoS ADPRT activity, identified LMWG-protein substrates of T3S-T-ExoS $(63,65,153)$ were examined for modification in the different cell lines. Cellular substrates examined in these studies included Ras, RalA, Rab5, Rab7, Rab8, Rab11 and Rac1, and modification was determined based on a shift in molecular mass by SDS-PAGE, previously shown to relate to ExoS ADP-ribosylation $(63,65,153)$.

While Ras appeared to be modified similarly in the human epithelial and murine fibroblasts, based on SDS-PAGE analyses, a difference was detected in the modification of the other LMWG-proteins between the two cell types (Figure $13 \mathrm{~A}$ ). Two modified forms of RalA were detected in HT-29 and LNCaP cells, but only one modified form of RalA was detected in NIH-3T3 or Swiss-3T3 fibroblastic cell lines. Comparisons of the ADP-ribosylation of Rab proteins by T3S-T-ExoS, found Rab5, Rab7, Rab8 and Rab11 to be modified in the human epithelial cell lines, but no modification of Rab proteins was detected in murine fibroblasts. (Representative results of Rab5 and Rab8 are shown in Figure 13 A.) Also, Rac1 modification was detected in human epithelial but not murine fibroblastic cells. The different number of modification bands observed in 


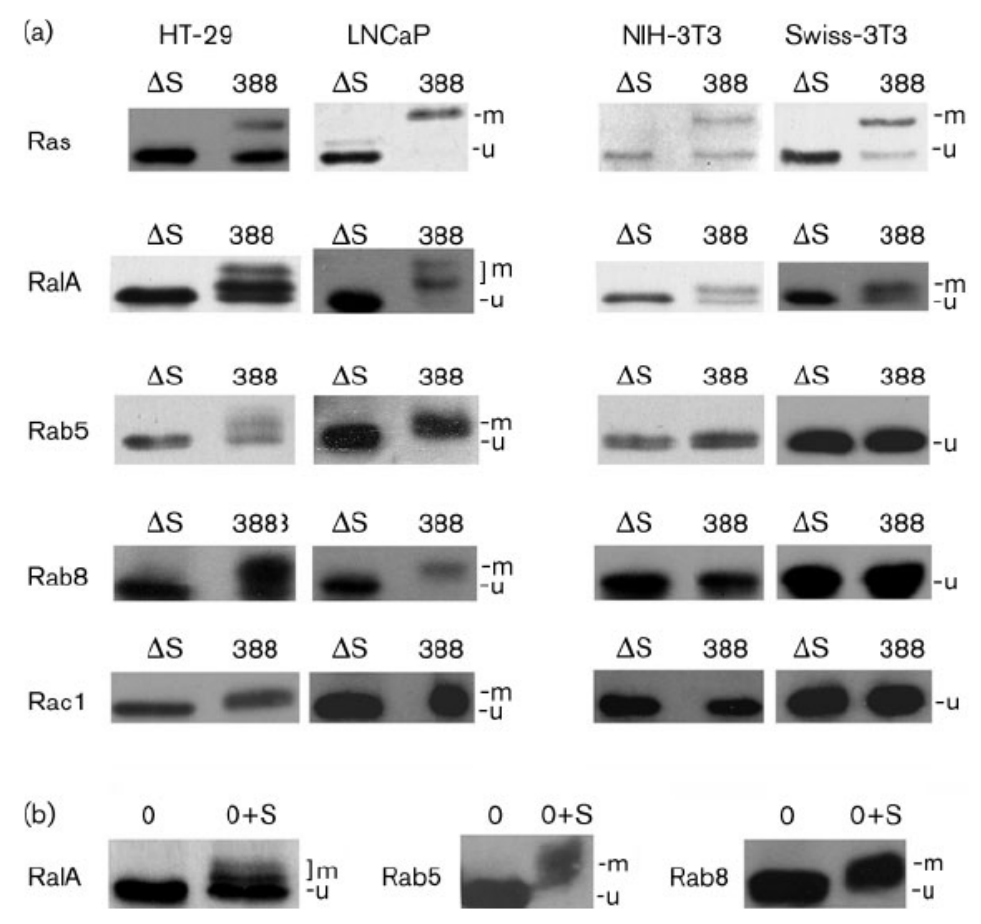

Figure 13. Cell line-related effect of substrate modification by T3S-T-ExoS.

(a) ADPRT substrate modification by T3S-T-ExoS: Cellular substrate modification was examined following a 4 to 5 hour co-culture period with strain 388 or $388 \Delta S(\Delta S)$, as in Figure 1. To analyze Ras modification, cells were lysed in TBS-TDS, Ras was immunoprecipitated using Y13-259 anti-pan Ras antibody, and immunoprecipitates were resolved on SDS 12.5\%-PAGE. Ras was detected by immunoblot analyses and visualized using enhanced chemiluminescence (ECL). To analyze the modification of RalA, Rab5, Rab8 and Rac1, cells were lysed in 1x Laemmli sample buffer and lysates resolved by SDS 12\%- PAGE. The individual LMWG-proteins were also detected by immunoblot analyses (b) Substrate modification by ExoS in an in vitro ADPRT reaction: ADP-ribosylation of fibroblastic RalA, Rab5 and Rab8 was examined in vitro using 0.2 $\mu \mathrm{M}$ purified ExoS, $10 \mathrm{mM}$ NAD and $40 \mu \mathrm{l}$ of NIH-3T3 or Swiss-3T3 fibroblast cell lysate as a source of both the endogenous protein and the 14-3-3 cofactor, in a $100 \mu$ l reaction volume. Reactions were incubated for 30 minutes at room temperature and stopped with $4 x$ Laemmli sample buffer and heating at $95^{\circ} \mathrm{C}$ for 5 minutes. Proteins were resolved by SDS-12\% PAGE and immunoblotted. Positions of ADPRT modified (M) and unmodified $(U)$ proteins are indicated, and immunoblots are representative of a minimum of 3 independent experiments. 
individual LMWG-proteins was previously shown to relate to the sequential addition of ADP-ribose moieties $(63,65)$.

To assess whether differences in patterns of substrate modification in these cell lines related to intrinsic protein differences or in situ cellular influences, RalA, Rab proteins and Rac1 from fibroblast cell extracts were incubated with purified ExoS in an in vitro ADPRT reaction. As shown in Figure $13 \mathrm{~B}$, fibroblastic derived RalA, Rab5, Rab8 (and Rac1, not shown) were able to be ADP-ribosylated by purified ExoS in vitro as efficiently as their respective epithelial proteins were in vivo. Also, because ADPRT reactions were performed using fibroblastic extracts as a source of eukaryotic cell proteins, neither the absence of functional 14-3-3 eukaryotic co-factor, nor the presence of endogenous ADPRT inhibitors appeared to be contributing to the limited ADPRT substrate modification detected in murine fibroblasts.

\section{Time-course analysis of T3S-T and substrate modification by ExoS ADPRT}

activity. To further explore whether the limited substrate specificity of fibroblastic cells related to less efficient T3S-T of ExoS into cells, LMWG-protein substrate modification was monitored relative to the internalization of ExoS ADPRT activity into the eukaryotic cell in a time-dependent study. In these experiments, cytosolic and TX-100 solubilized membrane fractions of cells were analyzed for ExoS ADPRT activity and RalA, Rab5, Rab8 and Rac1 modification following a 3, 4.5, or 6 hour exposure to bacteria. Our studies examined ExoS ADPRT activity, rather than the ExoS protein, because of our ability to quantify ExoS ADPRT activity precisely and because of previous difficulties in detecting ExoS protein by immunoblot analyses (64). 
Consistent with the studies shown in Figure $13 \mathrm{~A}$, patterns of LMWG-protein substrate modification in human epithelial and murine fibroblastic cells remained distinctly different, even with increasing time of exposure to bacteria (Figure $14 \mathrm{~A}$ ). The difference in substrate modification was specifically recognized in the single modification of RalA and absence of modification of Rab5, Rab8 or Rac1 in murine fibroblastic cells, compared with two modifications of RalA, two modifications of Rab5 and a single modification of Rab8 and Rac1 detected in human epithelial cells. Analysis of all cell lines was similar in that substrate modification was more efficient in the TX100 membrane fraction when compared with the cytosolic fraction. Also, while variations in levels of individual LMWG-proteins were detected between the cytosolic and membrane compartments, the relative distribution of these proteins was generally consistent in all cell lines. For example, there was a general preferential localization of RalA to the membrane fraction, Rab proteins were somewhat equally distributed in both the cytosolic and membrane fractions, and Rac1 preferentially localized to the cytosolic fraction. The lack of prominent differences in the cellular localization of LMWG-proteins is supportive of this not being a factor in differences observed in patterns of ExoS substrate modification in human epithelial and murine fibroblastic cells.

In time course analyses of ExoS ADPRT activity, increases in ADPRT activity were found to closely parallel increases in the efficiency of cellular patterns of substrate modification (Figure 14 B). ADPRT activity was significantly higher in the membrane than cytosolic fraction ( $p<0.009$, except for data received for HT-29 cells at $3 \mathrm{hrs}$ ), and progressively increased in both fractions with time in association with the increased efficiency of substrate modification. Notable in comparisons of ExoS ADPRT activity 
(a)

Epithelial cells
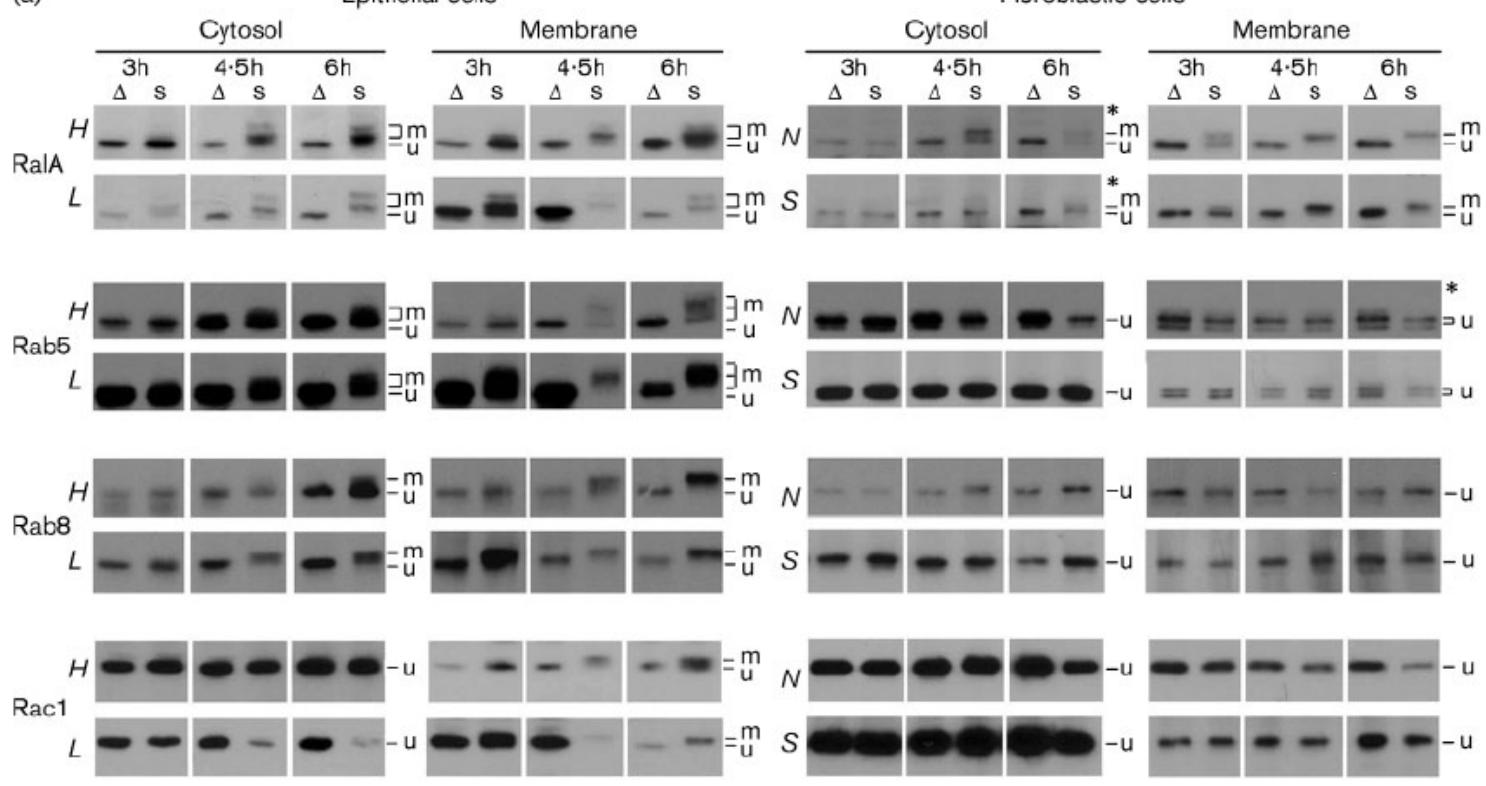

(b)

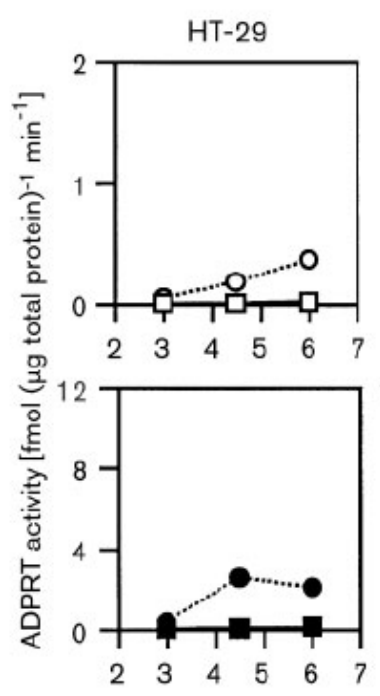

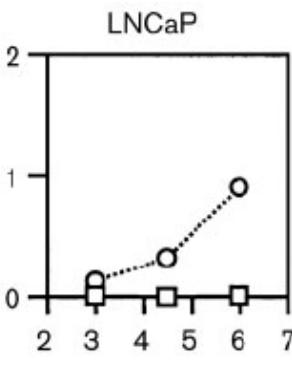
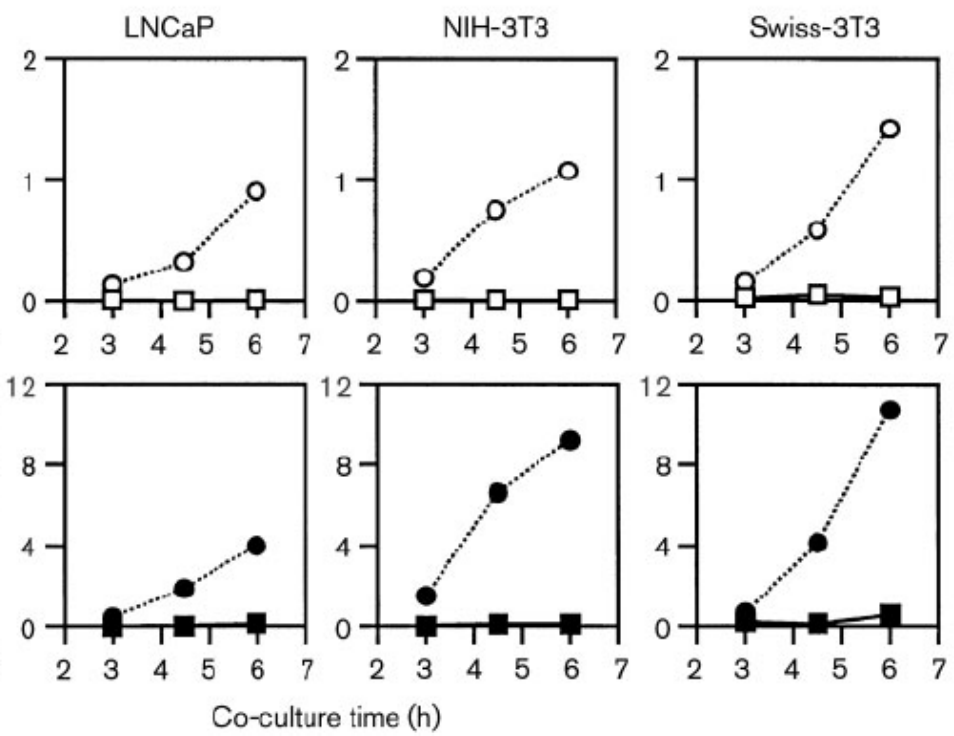

Figure 14. Time course analysis of ExoS substrate modification and internalization of ExoS ADPRT activity

(a) ADPRT substrate modification. Epithelial and fibroblastic cells were seeded in 100 $\mathrm{mm}$ dishes and co-cultured with strain $388 \Delta \mathrm{S}(\Delta)$ or $388(\mathrm{~S})$, as in Figure 1 , for $3,4.5$, or 6 hours. Cell monolayers were washed twice with PBS, non-adherent cells were recovered and combined with adherent, trypsin detached cells. The cells were washed 
with PBS, resuspended in $100 \mu \mathrm{l}$ of cell permeabilization solution (defined in Methods) and placed on ice for $10 \mathrm{~min}$ to release the cytosolic fraction. Permeabilized cells were centrifuged at $16,000 \mathrm{~g}$ for 20 minutes at $4^{\circ} \mathrm{C}$. The supernatant (cytosolic fraction) was removed, and the pellet was resuspended in $100 \mu \mathrm{l}$ of cell permeabilization solution containing $1 \% \mathrm{v} / \mathrm{v}$ Triton $\mathrm{X}-100$ and vortexed for 15 sec every 5 minutes, four times to solubilize membrane proteins. The membrane TX-100 soluble protein fraction was obtained by centrifugation at $16,000 \mathrm{~g}$ for $10 \mathrm{~min}$. To analyze substrate modification in the cytosolic and membrane fractions, $4 x$ Laemmli sample buffer was added to a $40 \mu \mathrm{l}$ aliquot of each sample, and RalA, Rab5, Rab8 and Rac1 modification was examined as described in Fig. 4. Positions of ADPRT modified $(\mathrm{m})$ and unmodified $(\mathrm{u})$ proteins are indicated. $(H)$ HT-29 cells; $(L)$ LNCaP cells; $(N)$ NIH-3T3 cells; $(S)$ Swiss-3T3 cells; $\left(^{*}\right)$ indicates a longer exposure to film, relative to the other samples, was required for the ECL signal shown.

(b) Internalization of ExoS ADPRT activity. ExoS ADPRT activity was quantified in the cytosolic and membrane fractions of HT-29, LNCaP, NIH-3T3 and Swiss-3T3 cells in a 1 hour in vitro ADP-ribosylation reaction, containing $1.2 \mu \mathrm{M}\left[{ }^{14} \mathrm{C}\right] \mathrm{NAD}$ and $100 \mu \mathrm{M}$ soybean trypsin inhibitor (SBTI) as the ADP-ribose acceptor. ADPRT activity is reported as fmol per min of ADP-ribose incorporated into SBTI per $\mu \mathrm{g}$ of cellular protein. ADPRT assays were performed in duplicate and the mean and SD (not evident) of samples represented in (a) are shown. The results are representative of 3 independent experiments. Open squares, cytosol, strain $388 \Delta S$; open circles, cytosol, strain 388; filled squares, membrane strain $388 \Delta S$; filled circles, membrane, strain 388 . 
was the high level of activity detected in both the cytosolic and membrane fractions of murine fibroblastic cells, even though more restricted patterns of substrate modification were detected. The high levels of ExoS ADPRT activity detected in fibroblasts further support the notion that ExoS activity is not tethered in murine fibroblasts by endogenous ADPRT inhibitors. While levels of ExoS ADPRT activity were calculated relative to total cellular protein concentration in Figure $14 \mathrm{~B}$, higher levels of activity were also evident in murine fibroblastic cells when related to total cell numbers (data not shown). In addition, while studies represented in Figure 14 were performed at an $\mathrm{MOI}$ of 10 , the same pattern of substrate modification was detected in murine fibroblasts at a $\mathrm{MOI}$ $>250$, highlighting the defined nature of murine fibroblastic cell substrate modification pattern. We conclude from these studies that the limited substrate modification by bacterially translocated ExoS in murine fibroblasts was not linked to the inefficient internalization of ExoS ADPRT activity by the $\mathrm{Pa}$ T3S system, but rather reflected in situ differences in ExoS function.

Patterns of ExoS substrate modification in different cell lines. To explore the specificity of the ExoS ADPRT substrate modification pattern relative to cell type or animal origin, we compared substrate modification by T3S-T-ExoS in fibroblasts, epithelial and endothelial cell lines from rodents, monkeys and humans (Table 1; Figure 15). Rodent-derived CHO-K1 and TCMK-1 epithelial cells, and RHEC endothelial cells, exhibited the same pattern of substrate modification as that of murine NIH-3T3 and Swiss-3T3 cells; that is, showing more limited modification of RalA and no modification of Rab proteins or Rac1. Simian-derived Cos-1 fibroblasts and Vero epithelial cells showed a similar pattern of substrate modification as human HT-29 and LNCaP cell 

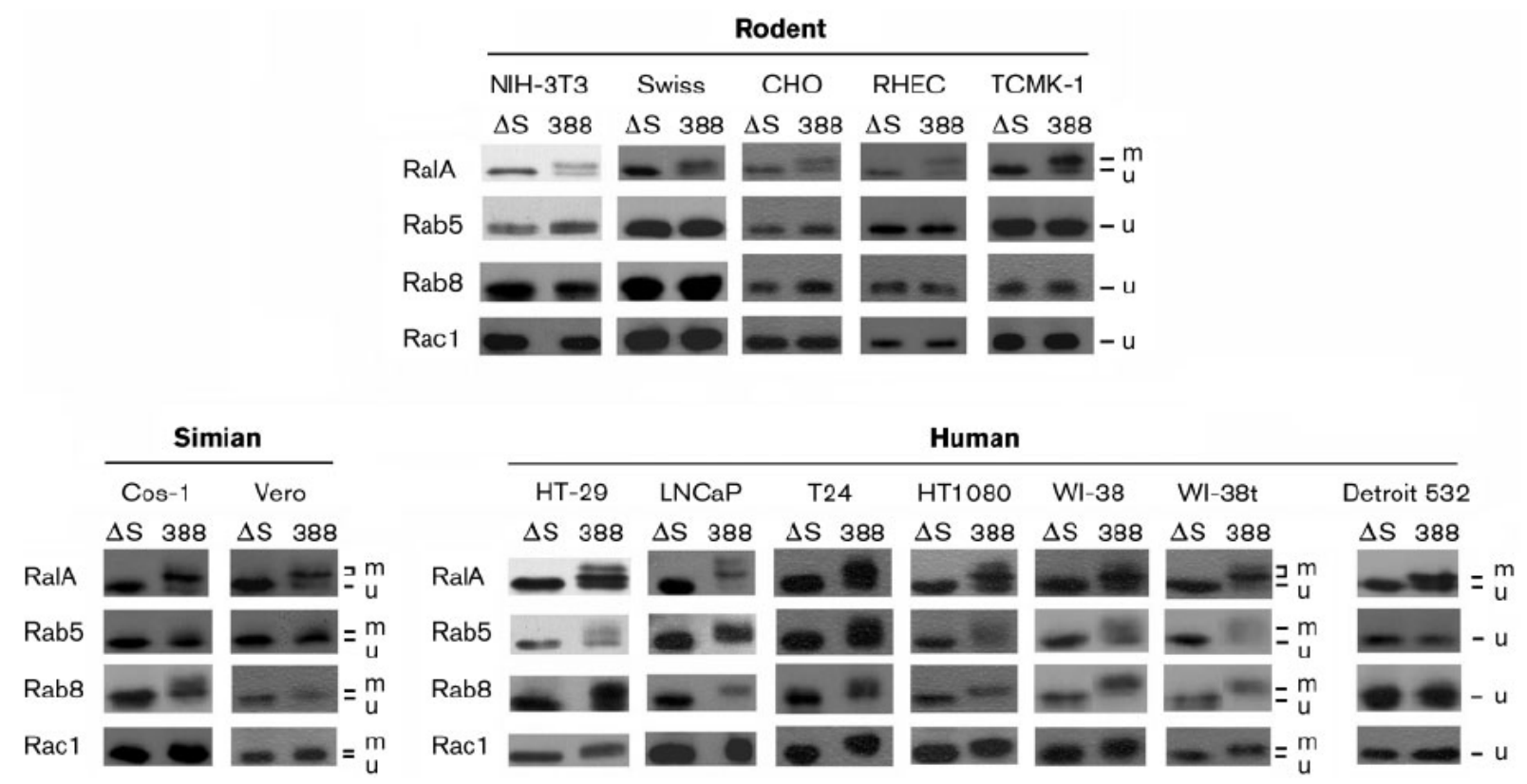

Figure 15. Patterns of LMWG-protein substrate modification by T3S-T-ExoS in different cell lines.

Eukaryotic cells were seeded at $10^{5}$ cells $/ \mathrm{ml}$ in 6 -well plates for 24 hours prior to coculture with $10^{6} \mathrm{cfu} / \mathrm{ml} 388,388 \Delta \mathrm{S}(\Delta \mathrm{S})(\mathrm{MOI} 10-20: 1)$, or no bacteria. After a 4.5 hour co-culture period, bacteria were removed, cells were washed in PBS and lysed in $200 \mu \mathrm{l}$ 1x Laemmli sample buffer. Lysates were resolved by SDS 12\%-PAGE and immunobloted for RalA, Rab5, Rab8 and Rac1, as described in Figure 4. ADPRTmodified $(\mathrm{m})$ and unmodified $(\mathrm{u})$ proteins are labeled. Immunoblots shown are representative of at least 4 independent studies. 
lines, although modification was consistently less efficient. Human-derived HT-1080 fibrosarcoma and T24 transitional cell carcinoma cells also displayed the same pattern of substrate modification as LNCaP and HT-29 cells. Since previous studies found tumor derived cells to be highly sensitive to the effects of ExoS (154), we examined whether oncogenic transformation or viral transformation of cell lines could influence substrate specificity. When substrate modification in normal and SV40-transformed human WI-38 fibroblasts (WI-38t) was compared, the pattern of substrate modification of both cell lines was similar to that of other human cell lines, indicating that viral transformation did not alter ExoS substrate modification. In addition, substrate modification by T3S-T-ExoS in the WI-38t cells did not differ from that detected in the HT-29, LNCaP, HT-1080 and T24 cells, indicating that neither viral nor oncogenic transformation influenced the substrate specificity of ExoS. The one cell line exception to the pattern of substrate modification observed for human cells was the Detroit 532 fibroblastic cells, which exhibited a more limited pattern of substrate modification, similar to that of rodent cell lines.

We conclude from these studies that patterns of ExoS substrate modification are linked more closely to the origin of the cell line, rather than cell type or state of cellular transformation. However, as evident from Detroit 532 human fibroblasts, this relationship is not absolute, and differences in individual cell lines can also alter ExoS substrate-modification patterns. 


\section{Discussion}

The T3S system is thought to be evolutionarily derived from bacterial flagellar machinery and was an adaptation that allowed the bacterium to directly translocate effector proteins and manipulate the function of eukaryotic cells (102). The conservation of the genetically complex T3S regulon among a broad range of Gram-negative organisms implies its general importance to bacterial survival. While it has been recognized that differences exist in the sensitivity of eukaryotic cells to T3S processes, the cellular mechanism for these differences, and the contribution of the eukaryotic cell to the T3S process remain largely unknown.

We have used the T3S-T of ExoS by Pa to explore how the host cell might influence the translocation and cellular effect of T3S-effector proteins. Previous studies found that macrophage and epithelial cell lines responded differently to the Pa T3S process, with cell morphology being affected in epithelial cell lines and cell viability being affected in macrophages (27). ExoS is the most extensively studied of the Pa T3S-effector proteins, and comparisons of different epithelial cell lines identified differences in cell sensitivity to T3S-T-ExoS (154). Notable in these comparisons was that epithelial cell sensitivity to ExoS appeared to parallel the opportunistic nature of $\mathrm{Pa}$ infections, implying a relationship between the T3S process and cells targeted by $\mathrm{Pa}$. Since studies supported that the host cell can influence the outcome of T3S-T events, we reasoned that we might be able to gain insight into these eukaryotic cell influences by comparing the cellular mechanism of ExoS in cell types that respond differently to T3ST-ExoS. 
We had previously observed differences in sensitivities of human carcinoma and mouse fibroblastic cells to the effects of T3S-T-ExoS. Based on these observations, we used HT-29 and LNCaP human epithelial cells and NIH-3T3 and Swiss-3T3 murine fibroblasts to explore how the host cell might contribute to differences in the toxicity of ExoS. Previously defined effects of ExoS on epithelial cells, which include inhibition of DNA synthesis, long-term cell rounding and loss of re-adherence (169), were used as criteria for functional comparisons of the toxicity of ExoS. Two strains of bacteria, 388 and $388 \Delta S$, were used to distinguish the effects of ExoS from other bacterial factors. Studies performed in parallel using strain $388 \Delta$ ExoT confirmed that the cellular effects being monitored related to ExoS, and not ExoT, which is also expressed by strain 388 (data not shown). Our studies identified individual cell line variations in the effects of ExoS on cell function, but overall, human epithelial cells examined were more sensitive than murine fibroblastic cells to the effects of ExoS on DNA synthesis, morphology and cellular adherence. Of these four cell lines, LNCaP epithelial cells were the most sensitive to the effects of T3S-T-ExoS, and Swiss-3T3 fibroblasts were the most resistant. We also found that decreased sensitivity to ExoS did not relate to decreased bacterial association.

In defining the mechanism for the differential effects of ExoS on cell function, our studies focused on ExoS ADPRT activity, rather than GAP activity, since previous studies linked toxic effects of T3S-T-ExoS on epithelial cell morphology and DNA synthesis to ExoS ADPRT activity (64). ExoS ADPRT activity was functionally evaluated based on the efficiency of ADP-ribosylation of cellular substrates. T3S-T-ExoS has been found to ADP-ribosylate multiple LMWG-proteins in HT-29 epithelial cells, with Ras and 
RalA modification detected first, followed by the modification of Rab proteins, then Rac1 (65). A different pattern of LMWG-protein substrate modification by T3S-T-ExoS was detected in murine fibroblasts. Although no differences in Ras modification were evident in the two cell types based on SDS-PAGE, differences were detected by twodimensional electrophoresis (2DE), with one modification of Ras detected in murine fibroblasts (E. A. Rucks, unpublished results), while two or three sites of Ras modification have been detected in human epithelial cells $(64,241)$. Differences in RalA modification were also detected, with only one modification of RalA detected in murine fibroblasts by SDS-PAGE, while two modifications were evident in human epithelial cells. In analyses of Rab proteins, Rab5, Rab7, Rab8 and Rab11 were all modified in human epithelial cells, while no modification of Rab proteins was detected in murine fibroblastic cell lines. Consistent with previous reports identifying LMWG-protein targets of ExoS ADPRT activity in NIH-3T3 fibroblast membranes (26), when murine fibroblastic cell extracts were used as a source of endogenous LMWG-proteins in in vitro ADPRT reactions using purified ExoS, RalA, Rab proteins and Rac1 were found to be modified by ExoS as efficiently as that observed in human epithelial cells. These results support the notion that the decreased efficiency of RalA, Rab, and Rac1 modification in murine fibroblasts did not relate to intrinsic protein properties, but rather to in situ cellular effects.

The decreased efficiency of LMWG-protein modification in murine fibroblasts suggested that the T3S process functioned differently in murine fibroblastic and human epithelial cells relative to either the efficiency of ExoS internalization or the action of ExoS once it was T3S-translocated. In comparing the efficiency of ExoS internalization 
in the two cell types, ExoS was found to be translocated as (or slightly more) efficiently in murine fibroblasts when compared to human epithelial cells, supporting that the T3S apparatus was functional in murine fibroblasts. Consistent with the translocation of ExoS through the membrane into cytosol via the T3S apparatus, levels of ExoS ADPRT activity and the efficiency of substrate modification was higher in membrane than cytoplasmic fractions of all cell lines, as has been previously recognized $(64,188)$. Our analyses of ExoS internalization relied on quantification of ExoS ADPRT activity, rather than ExoS protein, for we have had difficulty in detecting ADPRT-active ExoS within cells by immunoblot analyses using antibodies against ExoS (64). The more limited modification of LMWG-protein substrates in murine fibroblastic cells, in association with efficient ExoS internalization, indicated that cellular factors, beyond membrane translocation, were influencing the ADP-ribosylation of substrates by ExoS within the cell.

Differences in substrate modification between human epithelial and murine fibroblasts prompted us to examine how the pattern of ExoS substrate modification related to cell type, the animal origin of the cells, or the state of viral or oncogenic cellular transformation. In comparing ExoS substrate modification in murine, rat, hamster, simian or human cells, of epithelial, endothelial and fibroblastic cell types, two basic patterns of substrate modification were identified. One pattern showed more restricted substrate ADP-ribosylation, detecting limited modification of RalA and no modification of Rab proteins or Rac1. This pattern was common to rodent cell lines and independent of cell type. The second pattern showed more extensive LMWG-protein substrate ADP-ribosylation, with T3S-T-ExoS modifying RalA more efficiently and also 
modifying Rab proteins and Rac1. This pattern was apparent in human and simian cell lines, but modification in simian cell lines appeared less efficient. Notably, one human cell line, Detroit 532 fibroblasts, was found to have the more limited ExoS substrate modification pattern seen in rodent cells. The mechanistic link between the restricted ExoS ADPRT substrate pattern observed in Detroit fibroblasts is not known. In assessing the role of viral or oncogenic transformation in ADPRT substrate modification, no alterations in patterns were detected in any of the cell lines in association with cellular transformation.

The cellular mechanism for the different patterns of ADPRT substrate modification is not yet understood. Factors that could alter the substrate specificity of T3S-T-ExoS include differences in the subcellular targeting or localization of ExoS within cells and/or differences in co-localization of the ExoS eukaryotic cofactor, 14-3-3 protein. Relative to the functional co-localization of the 14-3-3 cofactor, based on our in vitro ExoS ADPRT reactions, we know that the cofactor is present and functional in murine fibroblastic cell extracts which exhibit the more restricted cellular substrate-modification pattern. It has also been found that 14-3-3 protein co-localizes to endosomes with Rab4 (15), although Rab4 does not appear to be a substrate of ExoS when internalized by the T3S process in either epithelial cells (65) or fibroblasts (E. A. Rucks, unpublished observation). These data imply that co-localization of $14-3-3$ co-factor by itself is not sufficient for ExoS substrate ADP-ribosylation. An alternative, more likely explanation for altered patterns of ExoS ADPRT substrate modification, based on current available data, is that differences in targeting of ExoS within the cell affect its ADPRT substrate specificity. Consistent with this hypothesis, both an $\mathrm{N}$-terminal processing site and a membrane 
localization domain have been identified within the first 78 amino acids of ExoS (176, 177). These sites appear to influence the subcellular localization of ExoS and may also prove to influence the substrate targeting specificity of ExoS ADPRT activity.

In relating substrate patterns of ADP-ribosylation by T3S-T-ExoS to effects of ExoS on cell function, ExoS has previously been found to affect the function of several of its LMWG-protein substrates. The ADP-ribosylation of Ras by ExoS is known to interfere with guanine nucleotide exchange factor (GEF) catalyzed GDP to GTP exchange, and the interaction of Ras with its downstream effector Raf- 1 and activation of the MAPkinase, Erk-2 $(73,91,241)$. In addition, the modification of Ras has been found to correlate with the ExoS-dependent inhibition of DNA synthesis (153). RalA is involved in signaling processes that affect cell proliferation, vesicular transport and cytoskeletal structure $(50,158,226,229)$. Recent studies have shown that the ADP-ribosylation of RalA by ExoS interferes with RalA interaction with its downstream effector, Ral binding protein 1 (63). Rab proteins control distinct pathways in endocytosis and vesicular trafficking which affect membrane recycling (194). In vitro analysis found the ADPribosylation of Rab5 by ExoS to interfere with its ability to bind early endosomal autoantigen 1 (EEA1), which retarded endosome-endosome fusion (10), which, in turn, could influence the regeneration of adherence procces. Together, these data implicate the potential role for ExoS-associated alterations of Ras, RalA and Rab protein function to contribute together to the cellular effects of T3S-T-ExoS.

Consistent with a functional relationship between substrate modification and effects of T3S-T-ExoS on cell function, LMWG-protein ADP-ribosylation was more extensive in human HT-29 and LNCaP epithelial cells, in association with their greater sensitivity to 
effects of ExoS on cell growth, morphology and re-adherence, when compared to murine fibroblasts. However, while a direct relationship was observed between efficiency of substrate modification and increased sensitivity to the effects of T3S-TExoS, an absolute link between patterns of substrate modification and specific cellular effects of ExoS was not evident, based on analyses used in our studies. For example, effects on DNA synthesis, cell rounding and re-adherence, which were associated with human epithelial cell responsiveness to ExoS were also detectable in $\mathrm{NIH}-3 \mathrm{~T} 3$ cells, which had more limited ExoS substrate modification; albeit the responses of NIH-3T3 to T3S-T-ExoS were less severe. We suspect that an inability to observe a direct relationship between functional effects of ExoS and specific patterns of substrate modification reflects the functional interrelationship of the LMWG-proteins targeted by ExoS within the cell. The function of Ras, Ral, Rac and Rab proteins are all linked to each other through a hierarchy of cellular network interactions. It therefore might be predicted that a more limited pattern of Ras and Ral modification could equate to cellular effects associated with more extensive patterns of substrate modification if given more time or upon more prolonged exposure to ExoS. Such a dose-response functional relationship of ExoS was evident in cellular re-adherence studies in which $\mathrm{NIH}-3 \mathrm{~T} 3$ cells required a longer exposure to ExoS-producing bacteria than HT-29 cells to obtain a similar loss of adherence (Figure 11). While the complexity of cellular signaling events might mask distinct relationships between ExoS substrate modification and effects on cell function, what is clearly evident in comparisons of different cell lines is that the host cell can influence the efficiency of the intracellular targeting of ExoS ADPRT activity. 
We conclude from these studies that the cellular response to T3S-T-ExoS is dictated to varying degrees by the host cell targeted by Pa. ADPRT substrate modification by T3S-T-ExoS is more restricted in rodent cells, when compared to human and simian cells, yet the T3S-T process appears equally efficient in these cells. It becomes apparent from these studies that the host cell is not a passive recipient during the bacterial driven T3S process, but rather cell properties are able to influence the activity and targeting of T3S-effector proteins.

\section{Acknowlegements}

We thank Dara Frank for providing the bacterial strains used in this study and appreciate the receipt of cell lines from Carwile LeRoy and Daohong Zhou. We would also like to acknowledge the technical assistance of Sean Olson, Zachary Olson, and Georgette Emanuelli in this project. This work was supported by Public Health Services grants NIH-NIAID 41694 and NIH-NIAID 45569, and by the Medical University of South Carolina Institutional Research Funds. 


\section{Chapter 3: Characterization of an ExoS type III translocation-resistant cell line}

Contents of this chapter were published as a Note in the January 2005 (Volume 73) issue of Infection and Immunity, pages 638-643, and appears with permission from the American Society of Microbiology Press and with permission from the authors, E. A. Rucks and J. C. Olson. 


\begin{abstract}
Pseudomonas aeruginosa $(P a)$ ExoS is a type III secreted (T3S), bifunctional protein that inactivates eukaryotic cell function. The co-culture of $P a$ strains expressing ExoS with HL-60 myeloid cells revealed the cell line to be resistant to the effects of T3ST-ExoS. Differentiation of HL-60 cells with phorbol 12-myristate 13-acetate (TPA) rendered the cell line sensitive to ExoS. To understand the cellular basis for the alteration in sensitivity, undifferentiated and TPA differentiated HL-60 cells were compared for differences in bacterial adherence, T3S induction, and ExoS T3S-T. These comparisons found that ExoS was translocated more efficiently in TPAdifferentiated HL-60 cells than in undifferentiated cells. The studies support the idea that the eukaryotic cell has the ability to influence $\mathrm{Pa}$ T3S at the level of membrane translocation.
\end{abstract}




\section{Introduction}

The type III secretory (T3S) process allows the direct translocation of bacterial effector proteins into eukaryotic cells and is associated with the virulence of many Gram-negative bacteria $(70,102)$. Four virulence factors, ExoS, ExoT, ExoU and ExoY, are known to be translocated into eukaryotic cells by $\mathrm{Pa} T 3 S(258,260)$. Unlike many Gram-negative bacteria, both clinical and environmental $P$. aeruginosa isolates maintain the T3S system, implying the integral role of T3S to $P$. aeruginosa survival in general $(52,54,200)$. Also, consistent with its opportunistic life-style, normal, healthy epithelial barriers and monolayers remain resistant to $P$. aeruginosa infection and T3S $(59,115$, 154). The purpose of these studies is to further understand the cellular basis of host-cell resistance to $\mathrm{Pa}$ T3S using the type III effector, ExoS, as a tool to monitor T3S.

In analysis of human epithelial cells, bacterially translocated ExoS (T3S-T-ExoS) has been found to cause a general inactivation of host cell function, as recognized by effects on cell growth, adherence, morphology and phagocytic processes $(66,169,170$, 193). ExoS affects eukaryotic cell function through a bi-functional mode of action. The $\mathrm{N}$-terminal region of ExoS includes a GTPase activating (GAP) activity (77), which can affect eukaryotic cell morphology and phagocytosis $(178,193)$. The C-terminal region of ExoS includes an ADP-ribosyltransferase (ADPRT) activity that targets specific cellular proteins, including low molecular weight G- (LMWG-) proteins in the Ras superfamily $(30,63,65,89,153)$. The ADPRT activity of ExoS is required for the irreversible effects

of ExoS on cellular proliferation, re-adherence and morphology $(64,169)$.

This study focuses on the effects of T3S-T-ExoS on HL-60 myeloid cell function. The HL-60 cell line originated from peripheral blood leukocytes of a patient with 
promyelocytic leukemia (32). When treated with specific compounds, HL-60 cells can be induced to terminally differentiate into granulocytes or monocytes $(33,134)$. We found HL-60 cells to be resistant to the toxic effects of ExoS in their undifferentiated form. However, upon differentiation with TPA, HL-60 cells became sensitive to ExoS. The identification of a T3S-T-resistant cell line that can be induced to become T3S-Tsensitive provides a model system for studying eukaryotic cell factors that influence the establishment of a functional $\mathrm{Pa}$ T3S process. 


\section{Materials and Methods}

Bacterial strains and culture conditions. Pa strains used in this study include: i) the prototype ExoS producing strain, 388 (106), ii) an isogenic ExoS mutant $388 \Delta$ exoS $(388 \Delta S)(126)$, iii) strain $388 \Delta p o p D:: \mathrm{Tc}^{*}(388 \Delta \mathrm{PopD})$, which lacks production of the PopD T3S translocation protein (236), and strain 388exs1::Tn1 (388-11), which contains a Tn1 insertion in the pscC gene, predicted to encode an outer membrane component of the $\mathrm{Pa}$ T3S process $(60,119,258)$. All Pa strains were provided by Dara Frank (Medical College of Wisconsin, Milwaukee, WI). Strain PA103 $\Delta$ exoU,exoT::Tc (PA103 UUT), a derivative of strain PA103, which lacks production of T3S effectors, ExoU, ExoS, ExoT and ExoY (235), served as the host strain for production and T3S translocation of pUCP encoded hemagglutinin-tagged ExoS (ExoS-HA). ExoS-HA was provided by Joseph Barbieri (Medical College of Wisconsin, Milwaukee, WI).

To allow induction and T3S-translocation of ExoS, P. aeruginosa were cultured in T3S-induction medium (TSBD-N) (106) for 16 hours prior to co-culture with eukaryotic

cells. In preparation for co-culture, bacteria were diluted based on $\mathrm{OD}_{590}$ to $10^{7} \mathrm{cfu} / \mathrm{ml}$, as previously described (170) in RPMI 1640 medium (Gibco-BRL, Gaithersburg, MD) supplemented with $0.6 \%$ bovine serum albumin (Sigma, St. Louis, MO) (RPMI-BSA) and were added to eukaryotic cells at a multiplicity of infection (MOI) of approximately 30:1, unless otherwise indicated.

Eukaryotic cell culture. The non-adherent HL-60 human leukemia cell line was obtained from the American Type Culture Collection (ATCC, CCL 240, Manassas, VA) and maintained in RPMI 1640 medium containing $10 \%$ or $20 \%$ fetal bovine serum and $100 \mathrm{U} / \mathrm{ml}$ penicillin and $100 \mathrm{\mu g} / \mathrm{ml}$ streptomycin (Gibco-BRL) (RPMI-FBS) at $37^{\circ} \mathrm{C}$ in $5 \%$ 
$\mathrm{CO}_{2}-95 \%$ air. In preparation for culture with bacteria, $\mathrm{HL}-60$ cells were centrifuged for $10 \mathrm{~min}$ at $400 \mathrm{~g}$ and seeded at $5 \times 10^{5} \mathrm{cells} / \mathrm{ml}$ in either polystyrene tubes or tissue culture plates, 48 hours prior to the addition of bacteria (Costar, Cambridge, MA). HL-60 cells were differentiated into mature monocytes by the addition of $20 \mathrm{ng} / \mathrm{ml}$ TPA (phorbol 12-myristate 13-acetate) (Sigma) to cell culture medium and incubation for 48 hours (111) (TPA-HL-60). For co-culture studies, cell medium was replaced with RPMIBSA containing no bacteria or $10^{7} \mathrm{cfu} / \mathrm{ml}$ bacteria, then cultured for 3 to 6 hours, as indicated, and assayed for changes in cell function and internalization of ExoS, as described below.

\section{Effects of T3S-T-ExoS on cell function.}

i) Quantification of DNA synthesis. Following a 3 to 4 hour co-culture period, cells were washed with phosphate buffered saline (PBS) and pulsed for 20 hours with 1 $\mu \mathrm{Ci} / \mathrm{ml}$ of $\left[\right.$ methyl- $\left.{ }^{3} \mathrm{H}\right]$ thymidine $(25 \mathrm{mCi} / \mathrm{mmol}$; Amersham Life Sciences, Arlington Heights, IL) in RPMI-FBS containing $200 \mu \mathrm{g} / \mathrm{ml}$ gentamicin (Sigma) and $100 \mu \mathrm{g} / \mathrm{ml}$ of ciprofloxacin (Bayer, West Haven, CT) (RPMI-FBS-GC). DNA synthesis was quantified as previously described (170) and is reported as percent $\left[{ }^{3} \mathrm{H}\right]$ thymidine incorporation relative to non-bacterial treated cells.

\section{ii) Examination of cellular morphology by scanning electron microscopy}

(SEM). Prior to co-culture with bacteria, HL-60 cells were seeded at $10^{6}$ cells $/ \mathrm{ml}$ on 12 mm-diameter glass Thermanox coverslips (Nalge Nunc, Naperville, IL) and cultured for 48 hours in $35 \mathrm{~mm}$ tissue culture dishes, in the presence or absence of TPA. Following a 3.5 hour co-culture period, bacteria were removed; cells were fixed with $2 \%$ cacodylate glutaraldehyde for 30 min as a primary fixative then with $2 \%$ acqueous 
osmium tetroxide for $30 \mathrm{~min}$ as a secondary fixative. Samples were rinsed with distilled water, dehydrated with $100 \%$ ethanol and incubated with hexamethyldisilazane (Sigma) until dry. The samples were then mounted and sputter-coated with gold palladium (20$\mathrm{nm}$ coating) and examined using a JEOL SEM-LV5410 scanning electron microscope.

ADP-ribosylation of LMWG-proteins by T3S-T-ExoS. ADP-ribosylation of LMWG-proteins of undifferentiated or TPA-HL-60 cells was assessed based on altered protein mobility following a 4 hour co-culture period with strain $388,388 \Delta S$ or no bacteria, as previously described $(63,65,153)$ (Chapter 2 , Methods and Materials).

Bacterial association with HL-60 cells. Effects of TPA differentiation on bacterial adherence to HL-60 cells were assessed after a 4 hour co-culture period of HL-60 or TPA-HL-60 cells with strains 388 or $388 \Delta$ S, at a high MOI (100:1) to optimize detection of bacterial association. Bacteria were removed and cells were washed three times with HEPES buffered saline (HBS) (137 mM NaCl; 4 mM KCl; 10 mM HEPES, pH 7.4; 11 $\mathrm{mM}$ glucose). Bacterial association was quantified by lysing cells in PBS containing $0.25 \%$ Triton X-100, diluting samples $1: 1000$ and plating $50 \mu$ l of lysate onto LB agar plates. Bacterial colonies were counted 24 hours later and the number of bacteria per eukaryotic cell was calculated.

Monitoring induction $\mathrm{Pa}$ T3S process. To assess T3S induction, strains 388 and $388 \triangle \mathrm{PopD}$ were cultured in a non-T3S induction medium (TSBD supplemented with 5 $\mathrm{mM} \mathrm{CaCl} 2$ ) prior to co-culture with eukaryotic cells. Undifferentiated or TPA-HL-60 cells were then co-cultured with or without bacteria for 4 hours, in Hanks Balanced Salt Solution (Sigma), which was found to minimize background induction of PopD and maintain eukaryotic and bacterial cell growth. After a 4 hour co-culture period, Laemmli 
sample buffer was added to co-culture wells, to harvest both, i) cell pellets containing eukaryotic cells and associated bacteria, and ii) co-culture supernatants containing bacteria and secreted proteins, within the same sample. Samples were heated at $95^{\circ} \mathrm{C}$ for 5 min, resolved by SDS-PAGE, immunoblotted using an anti-PopD antibody (provided by Dara Frank), and visualized by ECL. Comparable eukaryotic cell growth and protein loading were confirmed by immunoblotting for the eukaryotic housekeeping enzyme glyceraldehyde 3-phosphate dehydrogenase (GAPDH) (Chemicon International Inc., Temecula, CA).

\section{T3S-T of ExoS into HL-60 cells.}

i) Detection of T3S-T-ExoS ADPRT activity. Undifferentiated and TPA-HL-60 cells were co-cultured with strains 388 or $388 \Delta \mathrm{S}$ for $3,4.5$ or 6 hours, at a low MOI $(<10: 1)$ to facilitate detection of differences in the efficiency of ExoS translocation. Bacteria were removed, cells were washed, treated with $0.5 \%$ trypsin/0.53 mM EDTA (trypsin-EDTA) (Gibco-BRL) to proteolyze extracellular ExoS, then were lysed in $0.2 \%$ saponin, 100 $\mathrm{mM} \mathrm{NaCl}, 250 \mathrm{mM}$ sucrose, $5 \mathrm{mM}$ EDTA, containing the protease inhibitor, P8340 (Sigma). Cellular lysates were centrifuged at $16,000 \mathrm{~g}$ for $20 \mathrm{~min}$ to obtain the cytosolic (supernatant) fraction. The TX-100 soluble membrane fraction was obtained by treating cellular pellets with $1 \%$ TX-100 and vortexing for 15 sec every 5 min for 20 min, then centrifuging at $16,000 \mathrm{~g}$ for $10 \mathrm{~min}$. ExoS ADPRT activity was quantified in an in vitro ADPRT reaction, using soybean trypsin inhibitor (SBTI) as substrate, as previously described $(54,117,186)$. ExoS ADPRT activity in cellular fractions was calculated relative to total protein determined using the Sigma Diagnostics Lowry-based protein assay (Sigma). To compare ExoS ADPRT activity to substrate modification, an aliquot 
of each fraction was analyzed by SDS-PAGE and immunoblotted for RalA, Rab5 and Rac1, as described previously.

ii) Detection of T3S-T-ExoS protein. T3S-T-ExoS was detected using HA-tagged ExoS (ExoS-HA). In these studies, undifferentiated and TPA-HL-60 cells were cocultured for 4 hours with strain PA103AUT expressing ExoS-HA. Cells were washed and lysed in a digitonin extraction buffer $(0.01 \%$ digitonin, $10 \mathrm{mM}$ Pipes, $300 \mathrm{mM}$ sucrose, $100 \mathrm{mM} \mathrm{NaCl}, 3 \mathrm{mM} \mathrm{MgCl} 2,5 \mathrm{mM}$ EDTA) and separated into cytosolic and membrane fractions by centrifugation at $16,000 \mathrm{~g}$ for $15 \mathrm{~min}$ at $4^{\circ} \mathrm{C}$. Cytosolic and membrane fractions were equilibrated relative to volume in Laemmli sample buffer, samples were resolved by SDS-PAGE, immunoblotted using $2 \mu \mathrm{g} / \mathrm{ml}$ anti-HA antibody (Upstate Biotechnology, Lake Placid, NY) and visualized using ECL. Comparable eukaryotic cell protein loading was assessed by immunoblotting for GAPDH. 


\section{Results}

Induction of HL-60 cell sensitivity to ExoS. As part of a series of experiments examining the effects of T3S-T-ExoS on different cell types, the effects of ExoS on HL60 cell function were assessed in co-culture studies comparing $P a$ strain 388 and the isogenic non-ExoS producing strain, $388 \Delta S$. Using $\left[{ }^{3} \mathrm{H}\right]$ thymidine incorporation as a measure of cellular proliferation (170), exposure to Pa bacteria was found to cause an approximate $45 \%$ inhibition of HL-60 DNA synthesis. However, no ExoS specific effect on DNA synthesis was detected (strain 388 caused a $45.0 \% \pm 2.0 \%$ and strain $388 \Delta S$ caused a $44.7 \% \pm 2.0 \%$ inhibition). Unlike epithelial cells, HL-60 cells have a round morphology, which precludes the use of cell rounding as a measure of the effects of ExoS on cell morphology. Instead, scanning electron microscopy (SEM) was used to examine whether ExoS alters cell surface structures. We previously detected that T3ST-ExoS caused microvilli effacement in HT-29 epithelial cells (169). After a 3.5 hour coculture period, no alteration in HL-60 cell surface structure was evident following treatment with strain 388 (Figure $16 \mathrm{~A}$ ). The lack of ExoS-specific effects on DNA synthesis and cell morphology suggested that HL-60 cells were resistant to the effects of T3S-T-ExoS. 


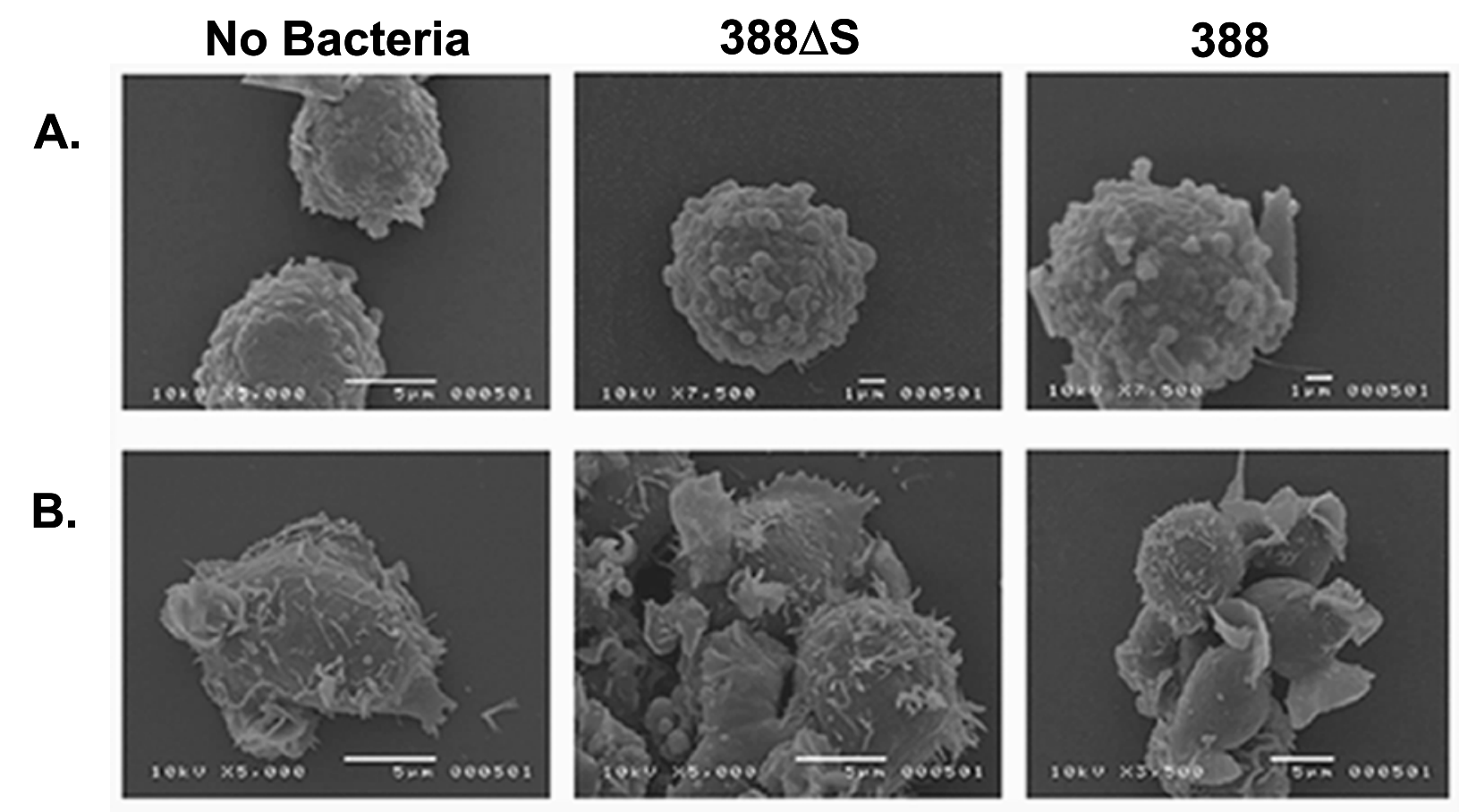

Figure 16. SEM analysis of the morphological effects of ExoS on HL-60 or TPAHL-60 cells.

HL-60 cells were seeded on glass cover slips in $35 \mathrm{~mm}$ tissue culture dishes and cultured for 48 hours in the absence (A) or presence (B) of $20 \mathrm{ng} / \mathrm{ml} \mathrm{TPA}$. Cell monolayers were washed and cultured with $388 \Delta S$, 388 or no bacteria for 3.5 hours. Bacteria were removed and cells were fixed, sputter coated with gold, and examined using a JOEL SEM-LV5410 scanning electron microscope. Noted in comparisons of these images is the loss of microvilli in TPA-HL-60 cells upon exposure to ExoSproducing bacteria. 
To assess whether HL-60 cell-sensitivity to ExoS could be altered by treatments that induce differentiation, cells were terminally differentiated into adherent monocytes by treatment with TPA, then co-cultured with strains $388,388 \Delta \mathrm{S}$ or no bacteria, as above. Terminal differentiation of eukaryotic cells halts cellular proliferation, thereby precluding the examination of effects of ExoS on the cellular proliferation of TPA-HL-60 cells. In SEM analyses, exposure of TPA-HL-60 cells to ExoS producing strain 388 resulted in an increased loss of cell surface microvilli, when compared with TPA-HL-60 cells exposed to $388 \Delta S$ or no bacteria. The effect of strain 388 on cell-surface microvilli provided an initial indication that TPA differentiation altered HL-60 cell sensitivity to T3S-T-ExoS. Notably, cell membrane ruffling, which was induced in HL-60 cells by TPA-treatment, was still evident following exposure to ExoS-producing bacteria. These results support that TPA treatment induced alterations in HL-60 cell sensitivity to T3S-TExoS, but effects of ExoS on cell structure were preferentially targeted to microvilli projections.

TPA-induced alterations in HL-60 cell sensitivity to T3S-T-ExoS were also recognized at the level of ExoS ADPRT substrate modification. The ADP-ribosylation of LMWG-proteins by ExoS has previously been found to be lead to alterations in protein mobility, based on SDS-PAGE analyses $(30,63,65,89,153)$. When TPA-HL-60 cells were co-cultured with strain $388,388 \Delta S$ or no bacteria for 4 hours and analyzed for ADP-ribosylation of LMWG-proteins, shifts in the mobility of Ras, RalA, Rab proteins and Rac1 were apparent (Figure 17). In comparison, only a slight modification of Rab proteins was detected in undifferentiated HL-60 cells co-cultured with strain 388 . 


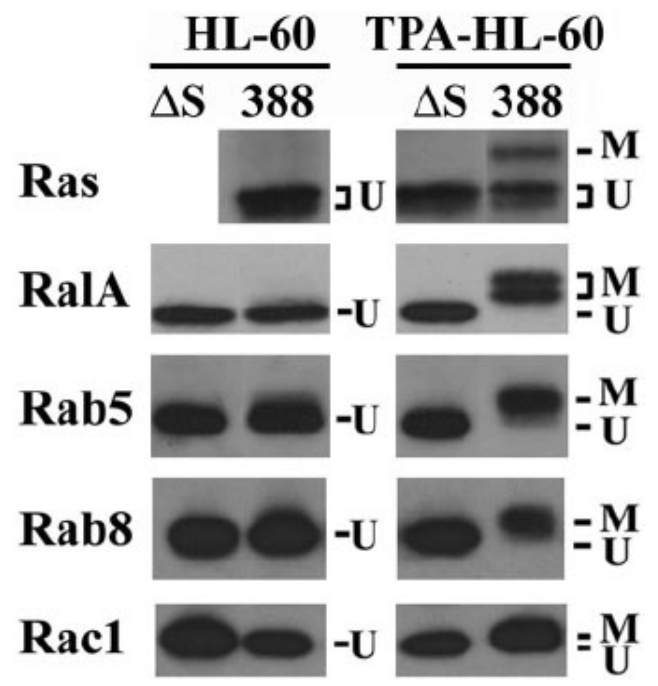

Figure 17. ExoS ADPRT substrate modification in HL-60 and TPA-HL-60 cells.

HL-60 cells were cultured for 48 hours in the presence or absence of TPA, prior to co-culture with strain $388 \Delta S(\Delta S)$ or 388 for 4 hours. Bacteria were removed, and cells were processed and analyzed for ADP-ribosylation of the indicated LMWGproteins based on altered mobility by SDS-PAGE and immunoblot analyses. Results are representative of at least 3 independent experiments. ADP-ribosylated (M) and unmodified $(U)$ proteins are labeled. 
The studies support that TPA differentiation induces HL-60 cell sensitivity to T3S-TExoS as evident in ExoS ADP-ribosylation of cellular proteins, which correlate with alterations in microvilli structure.

Mechanisms of induction of ExoS sensitivity. Although ExoS is secreted by $\mathrm{Pa}$ into culture supernatants, it is not toxic to eukaryotic cells in the absence of a T3S appartus $(170,258)$. Possible mechanisms by which TPA could induce alterations in sensitivity to ExoS include: i) indirect effects on T3S that facilitate bacterial adherence or non-T3S mediated diffusion of ExoS into cells, or ii) through direct effects on T3S that alter the induction or efficiency of the T3S-T process.

i) Indirect mechanisms. To examine whether TPA treatment induces cell surface alterations that enhance $\mathrm{Pa}$ adherence, rendering $\mathrm{HL}-60$ cells more sensitive to the bacterial contact-mediated T3S process, bacterial association to non-differentiated and TPA-HL 60 cells was assayed. In these studies, cells were co-cultured with strain 388 or $388 \Delta S$ for 4 hours at a high $\mathrm{MOI}(100: 1)$ to facilitate detection of bacterial association. Using a plating assay to quantify bacterial association, $25.8 \pm 2.6$ bacteria were found to associate per undifferentiated HL-60 cell, which compared with $8.5 \pm 0.8$ bacteria per TPA-HL-60 cell. In similar comparisons of 388 and $388 \Delta S$, bacterial association to eukaryotic cells was not significantly altered with ExoS expression (data not shown). The results indicate that TPA treatment did not affect HL-60 membrane properties in a manner that enhanced bacterial association with cells.

Another means by which TPA-treatment might indirectly affect ExoS internalization is through alterations in membrane properties that allow direct diffusion of ExoS into cells. To examine this possibility, TPA-HL-60 cells were cultured with soluble ExoS for 
4.5 hours in the presence and absence of bacteria. In addition to strains 388 and $388 \Delta S$, these studies included the T3S mutant strains, 388-11 (a PscC mutant defective in T3S-mediated secretion and translocation (258)) and 388 PopD (a PopD mutant defective in T3S-mediated translocation, but not secretion (236)) to assess ExoS diffusion independently of a functional T3S-process. Previous studies have shown that in the absence of PscC and PopD, ExoS is unable to exert toxic effects on eukaryotic cell function $(169,236)$. To allow an endogenous form of ExoS to be evaluated in these studies, strain 388 culture supernatant $(500 \mu \mathrm{l})$ containing $1.1 \mu \mathrm{g}$ of ExoS in the presence of other Pa secreted factors, served as the source of ExoS. Using ExoS ADPribosylation of cellular substrates to detect ExoS internalization, no substrate modification was detected in TPA-HL-60 cells cultured with soluble ExoS in the presence of strain $388 \Delta \mathrm{S}, 388-11$ or $388 \Delta \mathrm{PopD}$ (data not shown). In addition, no enhancement of ExoS substrate modification was detected when soluble ExoS was added to TPA-HL-60 cells co-cultured with strain 388 . The results support that TPA treatment does not alter HL-60 membrane integrity in a manner that allows ExoS internalization independently of the TTS process.

ii) Direct mechanisms. Differences in the sensitivity of undifferentiated and TPAHL-60 cells to ExoS might also relate to alterations in cellular properties that affect hostcell induction of the T3S process. Prior to coculture, strains 388 and $388 \Delta \mathrm{PopD}$ were cultured in medium that does not allow T3S induction (TSBD supplemented with $5 \mathrm{mM}$ $\mathrm{CaCl}_{2}$ ). Bacteria were then co-cultured with HL-60 or TPA-HL-60 cells for 4 hours in Hanks balanced salt solution (Sigma). Total PopD was examined by adding 4X Laemmli 


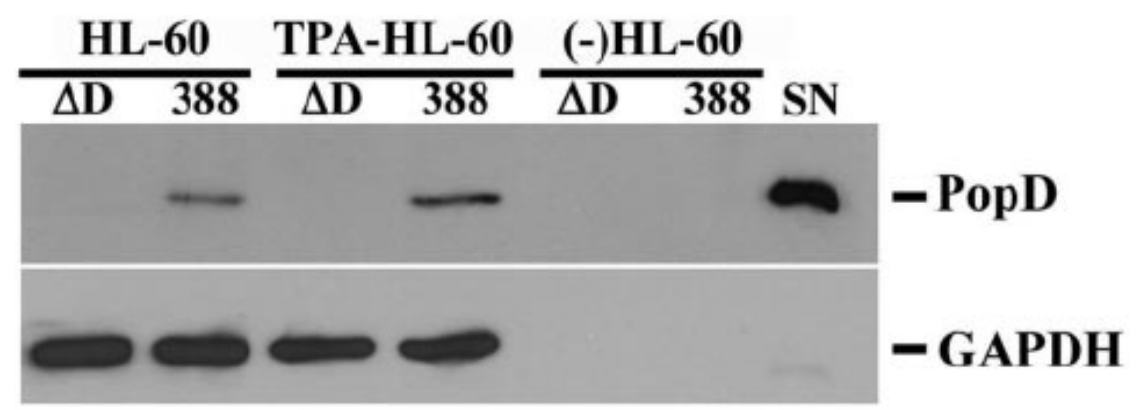

Figure 18. Analysis of PopD induction by HL-60 and TPA-HL-60 cells.

HL-60 cells were cultured in the presence or absence of TPA for 48 hours prior to co-culture with strains 388 or $388 \triangle \mathrm{PopD}(\triangle \mathrm{D})$ for 4 hours, then processed and resolved by SDS-PAGE and immunoblotted for PopD and GAPDH. Bacterial supernatant obtained from strain 388 cultured in inducing medium was used as a positive control (SN). Results are representative of at least three independent experiments. (-) HL-60, bacterial strains cultured in co-culture medium for 4 hours in the absence of eukaryotic cells. 
sample buffer (129) to tissue culture wells, which allowed the combined harvest of eukaryotic cells, bacteria and co-culture supernatants. Cells treated with strain $388 \triangle \mathrm{PopD}$, no bacteria or bacteria cultured in the absence of eukaryotic cells served as negative controls in these experiments. As shown in Figure 18, the induction of PopD by HL-60 cells closely corresponded to that of TPA-differentiated HL-60 cells. These results confirm that undifferentiated HL-60 cells are effective in inducing T3S components, which mediate T3S-T.

Collectively, these data point to the likelihood that the increased sensitivity of TPAHL-60 cells to ExoS relates to an increased efficiency of ExoS T3S-T. To assess the efficiency of ExoS translocation in HL-60 and TPA-HL-60 cells, time-course, co-culture studies were performed, examining ExoS ADPRT activity in cytosolic and Triton X-100 membrane soluble fractions. In these analyses, ExoS ADPRT activity was found to be 5-6 fold higher in both the cytosolic and TX-100 membrane fractions of TPA- HL-60 cells, as compared to that of undifferentiated HL-60 cells (Figure 19 A). The efficiency of ExoS ADPRT internalization was also found to directly correlate with the degree of ExoS ADPRT substrate modification (Figure 19 B). ADP-ribosylation of RalA, Rab5 and Rac1 was most efficient in the TX-100 membrane fractions of TPA-HL-60 cells, in association with the highest levels of ExoS ADPRT activity. Rab5 modification was less efficient and Rac1 was not modified in the cytosolic fraction of TPA-HL-60 cells in association with lower levels of ExoS ADPRT activity in these fractions. RalA modification could not be assessed since it was not detected in the cytosolic fraction. 

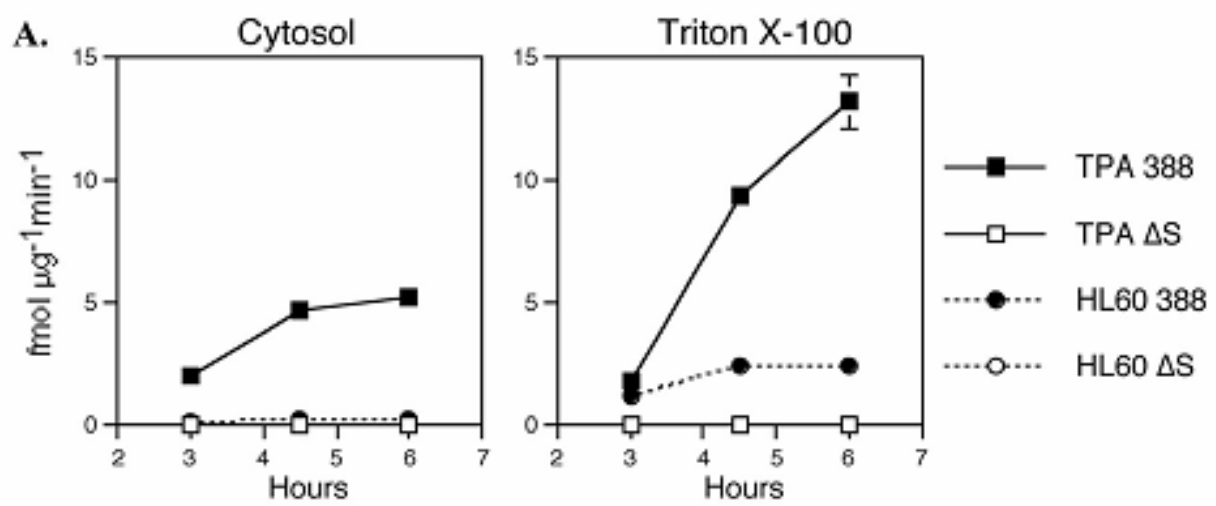

B.

HL-60 CELLS

TPA-HL-60 CELLS

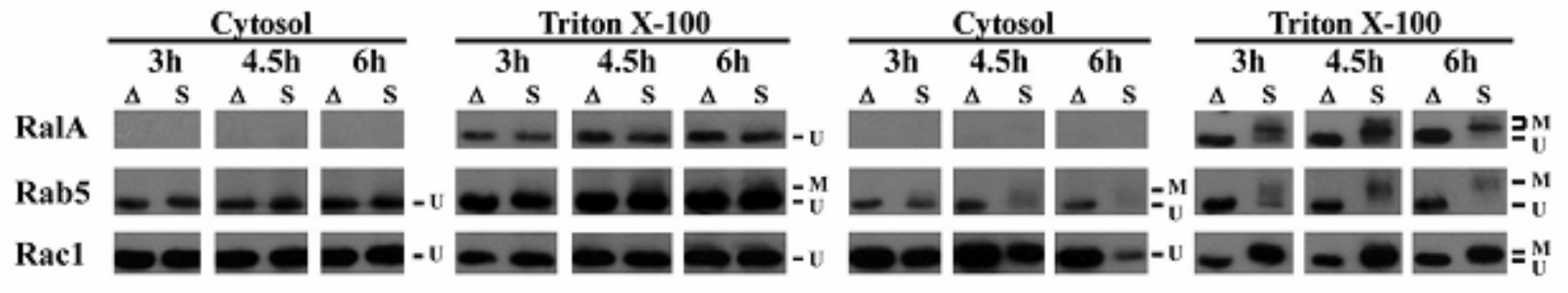

\section{Figure 19. Time-course analysis of T3S-T-ExoS ADPRT activity and substrate modification.}

HL-60 and TPA-HL-60 cells were co-cultured with strain 388 or $388 \Delta$ S for $3,4.5$ or 6 hours. Bacteria were removed, cells were washed, and cytosolic and Triton X-100 (membrane soluble) fractions were obtained, as described in Materials and Methods. $\boldsymbol{A}$. Internalized ExoS ADPRT activity. ADPRT activity is reported as fmol per min of ADPribose incorporated into the artificial substrate (SBTI) per $\mu \mathrm{g}$ of cellular protein, and the mean and standard deviation of assays performed in duplicate are shown. B. ExoS ADPRT substrate modification. Cell fractions in (A) were examined for ExoS ADPribosylation of eukaryotic LMWG-proteins by resolution by SDS-12\% PAGE and immunoblot analysis using RalA, Rab5 and Rac1 antibodies. ADP-ribosylated (m) and unmodified proteins $(\mathrm{u})$ are labeled. The results are representative of 3 independent experiments. 
Minimal substrate modification was detected in the membrane fractions of undifferentiated $\mathrm{HL}-60$ cells, as would be predicted based the limited ExoS ADPRT activity detected in these samples. Only a slight modification of Rab5 was detected after a 4.5 hour co-culture time. No ADP-ribosylation of cellular substrates was detected in the cytosolic fraction of undifferentiated HL-60 cells.

To further assess intracellular ExoS, undifferentiated HL-60 cells and TPA-HL-60 cells were co-cultured with Pa strain PA103 UUT expressing ExoS-HA (176). We have previously been unable to detect T3S-T-ExoS protein in eukaryotic cells by Western blot analysis $(64,197)$. However, the addition of the HA-tag to ExoS allowed detection of T3S-T-ExoS in eukaryotic cell extracts, without reported effects on ExoS function (176). Consistent with studies quantifying the internalization of ExoS ADPRT activity, ExoS protein was found predominately in the membrane fraction of TPA-HL-60 cells (Figure 20 A). Confirming TPA-HL-60 internalization of ExoS, the T3S-T-ExoS was detected in the cytosolic fraction of TPA-HL-60 cells. As shown in Figure $20 \mathrm{~B}$, levels of T3S-TExoS correlate with the efficiency of Rab5 ADP-ribosylation in the respective fractions. These results are consistent with the resistance of HL-60 cells to ExoS relating to the less efficient ExoS translocation, and that TPA differentiation induces cellular changes that allow a functional T3S apparatus to form and translocate ExoS. 


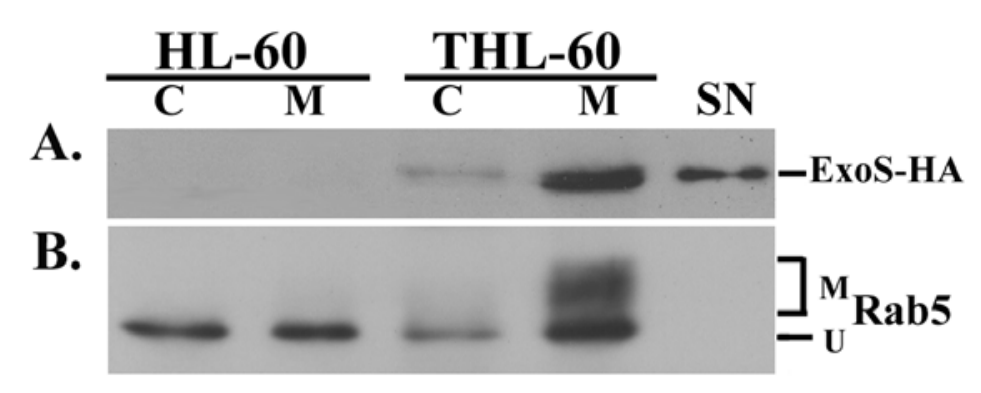

Figure 20. T3S-T of ExoS protein into HL-60 and TPA-HL-60 cells.

Cytosolic (C) and membrane (M) fractions of undifferentiated HL-60 or TPA-HL-60 cells were obtained by digitonin extraction and centrifugation. Fractions were resolved by SDS-PAGE and immunoblotted with (A) anti-HA antibody to detect ExoS-HA and (B) anti-Rab5 antibody to detect ExoS ADPRT substrate modification. Modified (M) and unmodified (U) Rab5 proteins are labeled. ExoS-HA, obtained from T3S-induced bacterial culture supernatants (SN), was included as a positive control. 


\section{Discussion}

It has become evident from studies of different cell lines that the host cell can influence the effectiveness of $\mathrm{Pa}$ T3S. Polarized confluent epithelial monolayers remain resistant to $\mathrm{Pa}$ infection and T3S, but become sensitive to both when the epithelial layer is compromised $(58,154,182)$. The host cell can also influence the toxicity and substrate targeting of T3S effectors, as evident in decreased ExoS toxicity, in association with alterations in LMWG-protein substrate modification patterns $(192,197)$. In studying the effects of ExoS on the promyelocytic HL-60 cell line function, we identified another cell line that was resistant to $\mathrm{Pa}$ T3S. A notable finding relative to HL60 cells was that sensitivity to T3S-T-ExoS could be induced by treatment with the phorbol ester, TPA.

Treatment of spherical, non-adherent HL-60 cells with TPA induces their differentiation into mature adherent monocytes that exhibit microvilli, pseudopodia and lamellipodia on their cell surface. Induction of HL-60 T3S-T-sensitivity by TPA treatment was initially recognized morphologically, by a loss of microvilli, with little or no change in expression of pseudopodia or lamellipodia following exposure to T3S-T-ExoS. Previous studies have linked effacement of microvilli to toxic effects of T3S-T-ExoS on actin cytoskeleton structure in epithelial cells and macrophages $(66,169,192)$. Functional effects of T3S-T-ExoS on microvilli structure in TPA-HL-60 cells correlated with the increased internalization of ExoS-HA and increased ExoS ADP-ribosylation of LMWGproteins. Our studies support that HL-60 resistance to T3S-T-ExoS relates to the lack of efficient ExoS translocation and corresponding ExoS ADPRT substrate modification, which can be induced by treatment with TPA. 
Several mechanisms were tested to explain the higher levels of T3S-T-ExoS in TPA-HL-60 vs. HL-60 cells. These included: i) alterations in the efficiency of bacterial adherence to HL-60 cells, which is required for T3S-T, ii) alterations in host cell induction of T3S proteins, or iii) TPA-induced membrane alterations that allow direct internalization of ExoS, independently of the T3S apparatus. None of these mechanisms accounted for the differences in the effects of ExoS on cell function and ADP-ribosylation of substrates in HL-60 and TPA-HL-60 cells. Another possible mechanism that might explain the decreased levels of T3S-T-ExoS in HL-60 cells is an increased rate of ExoS degradation. Studies examining this possibly also provided negative results. In in vitro ExoS ADPRT reactions, purified ExoS was found to efficiently ADP-ribosylate LMWG-proteins in HL-60 cell lysates, as previously reported (30), and ExoS was not degraded or its activity inhibited by endogenous HL-60 proteins (data not shown). Similarly, treatment of HL-60 cells or TPA-HL-60 cells with the irreversible proteasome inhibitor MG132 (Sigma) or protease inhibitors did not enhance T3S-T-ExoS ADPRT substrate modification in either cell line (data not shown). Alternatively, in support of HL-60 cell resistance to T3S-T-ExoS occurring at the level of membrane translocation, time course analyses revealed only a minimal increase in ExoS ADPRT activity in the membrane fraction of $\mathrm{HL}-60$ cells with time, and no activity was detected within the cytosol. In comparison, a linear increase in ExoS ADPRT activity was detected in the membrane fraction of TPA-HL-60 cells with time, and detectable but smaller amounts translocated into the cytosol. Similar results were obtained from tracking bacterial translocation of ExoS-HA. Combined, these data indicate that the differential sensitivity of HL-60 and TPA-HL-60 cells to ExoS reflect 
cellular influences on the $\mathrm{Pa}$ T3S-T, rather than cellular properties that affect membrane permeability, bacterial adherence, T3S-T-induction or ExoS degradation.

While these studies provided evidence that TPA differentiation of HL-60 cells altered cell line responsiveness to T3S-T-ExoS, the mechanism for this transition remains unknown. TPA induces HL-60 cells to differentiate into mature macrophages by activating protein kinase $\mathrm{C}$ (PKC) signaling pathways (232) and increasing protease activity (14). A hallmark of TPA differentiation is the ability of the cells to adhere to one another and to tissue culture substrates. This suggests the potential for TPA to induce alterations in HL-60 cell surface components that might affect T3S-responsiveness. Several receptors for $\mathrm{Pa}$ have been identified, including the cystic fibrosis transmembrane regulator (CFTR) $(75,180)$, asialo-GM1 $(34,41,87,123)$ and $\beta_{1}$ integrins $(182,195)$. However, the identity of the eukaryotic receptor that is required for $\mathrm{Pa}$ T3S-eukaryotic host cell interaction remains unknown. Interestingly, bacterial association with TPA-HL-60 cells decreases when compared to that of HL-60 cells, which might seem contradictory to the increased T3S-sensitivity of TPA-HL-60 cells. However, this could also be an indication that $P a$ adherence to TPA-HL-60 cells is more T3S-specific. Of note, candidate T3S-interactive proteins, such as $\alpha_{5} \beta_{1}$ integrins and intracellular adhesion molecules-1 (ICAM-1) show enhanced expression and avidity, respectively, in response to TPA differentiation $(14,131,195)$. Together, these draw attention to the potential use of comparisons of HL-60 and TPA-HL-60 cells to identify T3S-specific eukaryotic cell interactive components.

Currently, little is known about the host cell contribution to Pa T3S. Studies described here identify a link between HL-60 cell resistance to T3S-T-ExoS to host cell 
properties that prohibit the establishment of a functional T3S apparatus. While the mechanism for this T3S-resistance is not known, it appears to be reversed by treatments that affect cell dynamics and motility. Also of particular relevance to these studies is the identification of a T3S-resistant HL-60 cell line, which can be induced to become T3S-sensitive, and in so doing provides a model system to study eukaryotic cell mechanisms associated with alterations in Pa T3S-sensitivity.

\section{Acknowledgements}

We thank Dara Frank (Medical College of Wisconsin, Milwaukee, Wis.) for providing the Pa strains and PopD antibody and Joseph Barbieri (Medical College of Wisconsin) for providing the ExoS-HA construct. We thank Jennifer Fraylick Meredith, Claudia Rocha, and Timothy Vincent for their helpful comments during these studies, and we thank Carol Moskos for technical assistance with SEM. We also appreciate the editorial comments of Nyles Charon, Thomas Elliott and Richard Bakker.

This work was supported by Public Health Services grant NIH-NIAID 45569, by the Medical University of South Carolina Institutional Research Funds, and by the Mary C. Babb Cancer Center Foundation Fund 2V882 from West Virginia University. 
Chapter 4: Contribution of the host cell to the molecular targeting of type III translocated ExoS 


\section{Abstract}

Both the GTPase activating activity (GAP) and the ADP-ribosyltransferase activity (ADPRT) of bacterially translocated ExoS (T3S-T-ExoS) target low molecular weight Gproteins (LMWG-proteins) within the eukaryotic cell, which leads to interference of host cell function. We have identified differences in host cell responsiveness to ExoS, which now allows ExoS to be used as a tool to elucidate mechanisms by which the host cell can modulate $\mathrm{Pa}$ T3S effector function. Previous studies defined two main patterns of ExoS ADP-ribosylation: 1) a more restrictive pattern common to rodent cell lines, recognized as a less efficient modification of Ras and RalA, and 2) a more extensive pattern common to simian and human cell lines, recognized as a more efficient modification of Ras, RalA, Rabs 5, 7, 8 and 11, and Rac1. Residues 51-72 of ExoS define a membrane localization domain (MLD), which targets T3S-T-ExoS to eukaryotic membranes. Deletion of the MLD resulted in relocalization of ExoS to the cytosolic fraction. To examine the molecular basis for differences in ExoS substrate patterns, ExoS with mutations in its MLD ( $\triangle M L D)$, GAP (R146) and ADPRT (E379, E381) activities were examined for their influence on intracellular targeting of ExoS ADPRT activity. T3S-T-ExoS $\triangle M L D$ and T3S-T-ExoS-E381A produced a restrictive pattern of substrate modification in human cell lines, but the mutations did not severely alter the targeting of T3S-T-ExoS ADPRT activity in rodent cell lines. In comparison, T3S-TExoS-E379A was unable to ADP-ribosylate eukaryotic proteins. Mutation in the GAP region resulted in increased intracellular levels of T3S-T-ExoS, indicating the role of this region on translocation or clearance of ExoS. Together these studies identify molecular signals within ExoS that influence the targeting of its ADPRT activity and find that these 
signals are interpreted differently in human and rodent cell lines. To understand the mechanism for the differential targeting of ExoS ADPRT activity within human and rodent cell lines, T3S-T-ExoS was co-immunoprecipitated from human and rodent cells. Differences in T3S-T-ExoS protein interactions were observed, providing a means to understanding differences in T3S-T-ExoS intracellular trafficking mechanisms. 


\section{Introduction}

Pa utilizes the type III secretion (T3S) system to translocate the virulence factor, ExoS, into eukaryotic cells (258). T3S-translocation (T3S-T) of ExoS escalates tissue damage in an acute lung infection model (212) and inactivates cell function in a tissue culture model of infection (169). Specifically, in analysis of human epithelial cells, T3ST-ExoS inhibits cell growth and adherence and causes permanent cell rounding (66, 169). In J774 macrophages, T3S-T-ExoS protects $\mathrm{Pa}$ from phagocytosis (192). The complex, suppressive effects of T3S-T-ExoS on cell function are attributed to its bifunctional mode of action. The N-terminal portion of ExoS includes a GAP activity, which targets Rho, Rac and Cdc42, (77) and is required for the T3S-T-ExoS antiphagocytic phenotype (192). The C-terminal region of the molecule includes the ADPRT activity (117), which preferentially targets LMWG-proteins within eukaryotic cells $(63,65,89)$, and is required for the effects of T3S-T-ExoS on DNA synthesis, adherence and permanent cell rounding (64).

Subsequent studies have identified two main patterns of T3S-T-ExoS substrate ADP-ribosylation (197). The first, more restrictive, pattern is observed when ExoS is bacterially translocated into cells of rodent origin, which results in limited ADPribosylation of Ras and RalA. The second, more extensive, pattern is observed when ExoS is bacterially translocated into cells of human or simian origin, which results in more efficient ADP-ribosylation of Ras, RalA, in addition to ADP-ribosylation of Rab5, Rab8, Rab11, Rab7 and Rac1. These findings suggest that the eukaryotic cell influences the intracellular targeting of T3S-T-ExoS ADPRT activity. 
Integral to understanding the molecular mechanism of action of T3S-effector proteins is linking their functional activity to intracellular localization. To date little is known or understood about how T3S-T-ExoS is trafficked within the eukaryotic cell. Previous studies reported that T3S-T-ExoS ADP-ribosylates Rab proteins in a time course dependent manner (65). However, Rab proteins are not targeted in rodent cell lines, suggesting a link between assessiblity to vesicular trafficking mechanisms and the targeting of ExoS ADPRT activity. Other studies have indicated that transfected, enzymatically inactive ExoS is trafficked to the perinuclear region of eukaryotic cells (177). Recently, a membrane localization domain (MLD) was identified in the N-terminal region of ExoS, between amino acids 51 and 72 . This region targets T3S-T-ExoS to eukaryotic membranes (176). The effect of this domain on ExoS subcellular localization is consistent with studies, which found that T3S-T-ExoS ADPRT is enhanced in membrane fractions of eukaryotic cells $(64,188,197)$.

In order to ADP-ribosylate substrates, ExoS requires a eukaryotic factor, a member of the14-3-3 family of proteins (68). 14-3-3 proteins are ubiquitously expressed in all eukaryotes and are responsible for the subcellular localization, stability and molecular activity of over 100 target proteins (46). While it is unknown with which 14-3-3 isoform(s) T3S-T-ExoS interacts within the eukaryotic cell, all isoforms are capable of stimulating ExoS ADPRT in vitro (268). Differences in substrate modification patterns in rodent versus human cells may be linked to potential host cell influences on T3S-T-ExoS subcellular localization and differential trafficking patterns, either through the vesicular network or 14-3-3 interactions. The goal of these studies is to characterize differences in intracellular targeting of T3S-T-ExoS. 
We were able to explore the mechanism by which the host cell influences the intracellular activity of T3S-T-ExoS using MLD, GAP or ADPRT mutant forms of ExoS. Studies, which compare rodent and human cell lines, highlight the role of 1) the MLD in ExoS localization and substrate modification, 2) the GAP domain on T3S-T of ExoS, 3) the E379, E381 catalytic residues in ADPRT function, and 4) differences in protein interactions with T3S-T-ExoS in rodent and human cell lines. 


\section{Materials and Methods}

Bacterial strains and culture conditions.

$\begin{array}{cccccccc}\text { Strain } & \text { T3S } & \text { T3S- } & \text { ExoS } & \text { ExoT } & \text { ExoU } & \text { ExoY } & \text { Reference } \\ 388 & + & + & + & + & - & + & (106) \\ 388 \Delta S & + & + & - & + & - & + & (126) \\ 388 \Delta \text { PopD } & + & - & + & + & - & + & (235) \\ 38811 & - & - & + & + & - & + & (258) \\ \text { PA1030UT } & + & + & - & - & - & - & (235)\end{array}$

Table 2. Pa strain phenotypes

The + and - symbols indicate the presence or absence of the indicated phenotype, T3S, T3S-T or expression of the indicated T3S effector.

$P a$ strains used were cultured as described in Chapters 2 and 3 . Bacteria were added to eukaryotic cells at an $\mathrm{MOI}$ of 50-100. The phenotypes of the strains used in these studies are shown in Table 1. PA103AUT strains expressing pUCP plasmid encoded ExoS and ExoS with mutations in the GAP (R146A) and/or ADPRT (E379A or E381A) were constructed as previously described $(64,141,235)$.

\section{Construction of ExoS-HA mutants}

To assess how catalytic regions of ExoS affect its T3S-T, mutants of ExoS GAP and/or ADPRT activities were constructed using the pUCPExoS-HA template. pUCP vectors (211) encoding ExoS with an hemagglutinin tag (ExoS-HA) and ExoS with a deletion in the membrane localization domain (MLD) (residues 51-72) (ExoS $\triangle M L D-H A)$ were kindly provided by Joseph Barbieri (Medical College of Wisconsin, Milwaukee, WI) (176). The pUCPExoS-HA encodes a Pstl-BamHI fragment of ExoS cloned from strain $388(126,258)$. When pUCPExoS-HA is expressed in strain PA103AUT, it allows the 
T3S-T of ExoS in the absence of other known T3S effectors. The addition of the HA tag to ExoS allows T3S-T-ExoS to be detected within eukaryotic cells by Western blot analysis, without affecting ExoS function (176). The R146A GAP mutation was introduced to pUCPExoS-HA (pUCPExoS-R146A-HA), essentially as previously described (64), using the QuikChange PCR-based site-directed mutagenesis system (Stratagene, La Jolla, CA). The primers used to introduce the mutation (indicated in bold) and an Nhel restriction site (underlined) were as follows: forward, 5'CGGAGATGGGGCGCTAGCTTCGCTGAGCACCG-3', and reverse, 5'CGGTGCTCAGCGAAGCTAGCGCCCCATCTCCG-3'. PCR reaction mixtures contained $200 \mathrm{ng}$ of plasmid DNA, $25 \mu \mathrm{M}$ primers, $625 \mu \mathrm{M}$ mixture of deoxynucleotide triphosphate (dNTP's), $5 \mu$ l Pfu reaction buffer and $2.5 \mathrm{U}$ of Pfu DNA polymerase (Stratagene). The reaction mixtures were subjected to one denaturing cycle of 2 minutes at $95^{\circ} \mathrm{C}$, and then 18 cycles under the following conditions: 30 sec denaturing cycle at $95^{\circ} \mathrm{C}, 30 \mathrm{sec}$ annealing cycle at $63^{\circ} \mathrm{C}$ and a 12 minute extension cycle at $72^{\circ} \mathrm{C}$, and completed by a 1 hour extension cycle at $72^{\circ} \mathrm{C}$. The PCR product was digested with Dpnl, then purified using an ethanol precipitation protocol. The entire precipitate was then electroporated into electrocompetent Escherichia coli DH5a electrocompetent cells (24).

The E379A mutation was introduced into the pUCPExoS-HA vector to produce pUCPExoS-E379A-HA using the PCR-based GeneTailor Site Directed Mutagenesis system (Invitrogen, Carlsbad, CA). The primers used to introduce the mutation (bold) and an Bsml site (underlined) were as follows: forward, 5'GGGATATCGAACTACAAGAATGCAAAAGAGA-3', and reverse, 5'- 
CATTCTTGTAGTTCGATATCCCGCTGA-3'. The E381A mutation was introduced into both pUCPExoS-HA and pUCPExoS-R146A-HA using the GeneTailor Site Directed Mutagenesis system, resulting in the construction of pUCPExoS-E381A-HA and pUCPExoS-R146A/E381A-HA. The following primers were used to introduce the E381A mutation (bold) and a BsrG1 site (underlined): forward, 5'CAAGAATGAAAAAGCGATTCTGTACAACAAAGAAAC-3', and reverse, 5'CTTTTTCATTCTTGTAGTTCGATATCC-3'. To construct a pUCPExoS-E379A/E381AHA mutant, an E381A mutation was introduced to the pUCPExoS-E379A-HA template using the following primers: forward, 5'-

CTACAAGAATGCAAAAGCGATTCTGTACAACAAAGAAAC-3', and reverse, 5'TTTTGCATTCTTGTAGTTCGATATCC-3'.

For all mutants created by the GeneTailor Site-Directed Mutagenesis system, prior to PCR reactions $100 \mathrm{ng}$ of template DNA were methylated using 4 units of $\mathrm{CpG}$ Methylase (M.Sss I) (New England Biolabs, Beverly, MA). PCR reaction mixtures contained 12.5 ng methylated DNA, $300 \mu \mathrm{M}$ dNTP's, $10 \mu \mathrm{M}$ primers, $1 \mathrm{mM} \mathrm{MgSO}_{4}$, High Fidelity PCR buffer, and 1.5 units of Platinum Taq High Fidelity enzyme (Invitrogen). The reaction mixtures were subjected to one 2 minute denaturing cycle at $94^{\circ} \mathrm{C}$, followed by 35 cycles: $30 \mathrm{sec}$ denaturing cycle at $94^{\circ} \mathrm{C}$, a $30 \mathrm{sec}$ annealing cycle at $55^{\circ} \mathrm{C}$ and a 8 minute extension cycle at $68^{\circ} \mathrm{C}$, and completed by a final 10 minute extension cycle at $68^{\circ}$. The PCR reaction $(4 \mu \mathrm{l})$ was then transformed into One-Shot Max Efficiency DH5a-T1 ${ }^{\mathrm{R}}$ (Invitrogen), using a heat-shock method (13).

pUCPExoS-R146A/E379A-HA and pUCPExoS-R146A/E379A/E381A-HA were constructed by digesting plasmids, pUCPExoS-R146A-HA, pUCPExoS-E379A-HA and 
pUCPExoS-E379A/E381A-HA with enzymes Eagl (which cuts in the middle of exoS) and $\mathrm{BamHI}$ (which cuts outside the C-terminal region of exoS). Eagl-BamHI fragments from pUCPExoS-E379A-HA and pUCPExoS-E381A-HA were gel purified using the Wizard SV Gel and PCR Clean-Up system (Promega, Madison, WI) and ligated into the digested pUCPExoS-R146A-HA plasmid (100 ng vector; $115 \mathrm{ng}$ insert) using T4 DNA ligase (Promega). The ligation reaction ( $5 \mu \mathrm{l})$ was transformed into One-Shot Max Efficiency DH5a-T1 ${ }^{\mathrm{R}}$ (Invitrogen).

Mutants were selected by plating transformed E. coli strains onto LB agar plates containing $100 \mu \mathrm{g} / \mathrm{ml}$ ampicillin. Clones were initially screened using restriction digests for added sites. ExoS mutations were confirmed by sequencing (Biotech Core, Inc., Sunnyvale, CA). pUCP vectors (150 ng) encoding mutant forms of ExoS-HA were electroporated into electrocompentent PA103AUT (43). Transformed bacteria were selected on LB agar containing $400 \mu \mathrm{g} / \mathrm{ml}$ carbenicillin. Positive clones were tested for ExoS expression by growing cells in TSBD-N medium, harvesting cells and fractionating them into secreted supernatants and whole cell pellets. Samples were processed for SDS-PAGE and immunoblotted with anti-ExoS and anti-HA-tag antibodies (Covance, Berkeley, CA). To confirm that the transformed Pa contained the correct ExoS construct, plasmid was re-isolated from Pa using the Holmes and Quigley phenol/chloroform extraction protocol (97), transformed into $\mathrm{DH} 5 \alpha$, and subjected to restriction digest analysis.

\section{Eukaryotic cell culture}

Eukaryotic cells were cultured as described in Chapters 2 and 3. 


\section{Monitoring ADP-ribosylation of eukaryotic substrates}

\section{In vitro analysis.}

Eukaryotic cells were grown to confluency, washed twice with Dulbecco's phosphate buffered saline (DPBS) and lysed for 30 minutes on ice in a buffer containing 0.03 M HEPES ( $\mathrm{pH}$ 7.5), 1\% Triton X-100, $10 \mathrm{mM} \mathrm{NaCl}, 10 \%$ glycerol, 1 mM EGTA, 25 $\mathrm{mM} \mathrm{NaF}, 1 \mathrm{mM} \mathrm{Na}_{3} \mathrm{VO}_{4}, 10 \mathrm{mM} \beta$-glycerophosphate and supplemented with Protease Cocktail III (Calbiochem), proteosome inhibitor MG-132 (Sigma) and benzamidine, then stored at $-70^{\circ} \mathrm{C}$. Before the in vitro ADPRT reaction, lysates were thawed at room temperature, and unbroken cells and nuclei were cleared by centrifugation at 16,000 $\times g$ for 5 minutes at $4^{\circ} \mathrm{C}$. To monitor ExoS ADP-ribosylation of eukaryotic substrates, supernatants, which were the sources of ExoS and mutant ExoS, from T3S-induced bacterial cultures $(\sim 1.7 \mu \mathrm{g}$ of total protein) and $100 \mathrm{nM}$ NAD were added to the aliquoted cleared lysates. Mixtures were incubated for 1 hour at room temperature, and reactions were stopped by the addition of 4 X Laemmli sample buffer (129), and heating at $95^{\circ} \mathrm{C}$ for 5 minutes. Samples were resolved by SDS-12\% PAGE and immunoblotted for the appropriate proteins, as outlined in Chapters 2 and 3.

\section{In vivo analysis}

T3S-T-ExoS and T3S-mutant ExoS was determined as described in Chapters 2 and 3.

\section{Intracellular localization of T3S-T-ExoS}

\section{i) Co-culture}

With the ability to detect intracellular ExoS through immunoblot analysis, several cellular fractionation techniques were developed to track T3S-T-ExoS's cytosolic or membrane localization. To determine the intracellular localization of T3S-T-ExoS, 
eukaryotic cells were seeded in $100 \mathrm{~mm}$ dishes at $10^{5} \mathrm{cells} / \mathrm{ml}$ and grown to $60-80 \%$ confluency. Cells were co-cultured with Pa strains, expressing pUCPExoS-HA (MOI of 20:1), for 5 hours. At the end of the co-culture period, cells were washed twice with DPBS, and detached with $2 \mathrm{ml}$ 0.025\% Tryspin-EDTA (GibcoBRL, Gaithersburg, MD). The trypsin served to both detach eukaryotic cells and proteolyze extracellular ExoS (non-T3S-T-ExoS). After 12 minutes, cells were removed from the dishes and the trypsin was neutralized with cell medium containing FBS.

\section{ii) Ultracentrifugation method}

The membrane or cytosolic localization of T3S-T-ExoS was initially determined by subjecting co-cultured eukaryotic cells to ultracentrifugation. Washed and trypsinized cells were pelleted by low-speed centrifugation and resuspended in $650 \mu$ of HBIl buffer (3 mM Imidazole, pH 7.4, $250 \mathrm{mM}$ sucrose and protease inhibitors). Lysates were obtained by passaging cells through a 5/8 inch 27 gauge needle 14 times. Unbroken cells and nuclei were cleared by centrifugation at $800 \times \mathrm{g}$ for 5 minutes at $4^{\circ} \mathrm{C}$. Lysates were further fractionated into cytosolic (S100) and membrane (P100) fractions by ultracentrifugation at $116,140 \times \mathrm{g}$ for 40 minutes at $4^{\circ} \mathrm{C}$. The $\mathrm{P} 100$ fraction was equilibrated to the volume of the $S 100$ fraction, and $4 X$ Laemmli sample buffer was added to an aliquot of sample, which was processed for SDS-PAGE and immunoblotted for ExoS using an anti-HA antibody (Upstate, Lake Placid, NY). Additional aliquots of the same samples were examined for T3S-T-ExoS ADP-ribosylation of eukaryotic substrates, and ExoS ADPRT activity, as previously described in Chapters 2 and 3 $(197,198)$. 


\section{iii) Digitonin extraction}

As a means to track T3S-T-ExoS ADPRT activity, the subcellular localization of substrates ADP-ribosylated by ExoS was determined by digitonin extraction. After the co-culture period, cells were lysed in $750 \mu \mathrm{l}(60 \mathrm{~mm}$ dish) or $1 \mathrm{ml}$ (100 mm dish) with digitonin extraction buffer (0.01\% digitonin, $10 \mathrm{mM}$ Pipes, $300 \mathrm{mM}$ sucrose, $100 \mathrm{mM}$ $\mathrm{NaCl}, 3 \mathrm{mM} \mathrm{MgCl}$, $5 \mathrm{mM}$ EDTA; $\mathrm{pH}$ buffer to 6.8) for 20 minutes on ice. Cells were scraped into microfuge tubes and separated into cytosolic (supernatant) and membrane (pellet) fractions by centrifugation at $16,000 \times g$ for 15 minutes at $4^{\circ} \mathrm{C}$. The volumes of supernatants and pellets were equilibrated with sample buffer and processed as previously described for SDS-PAGE and immunoblot analysis.

\section{iv) Modified digitonin extraction}

A limitation of the ultracentrifugation method was that only 6 samples could be processed at one time. To increase the throughput of the fractionation analysis, a modified-digitonin extraction method was developed. Washed and trypsinized eukaryotic cells (described above) were pelleted by low-speed centrifugation and resuspended in $750 \mu \mathrm{l}$ modified digitonin extraction buffer (10 mM Imidizole, pH 7.4, 250 $\mathrm{mM}$ sucrose, $3 \mathrm{mM} \mathrm{MgCl}$, $5 \mathrm{mM}$ EDTA, $0.01 \%$ digitonin). (The new buffer recipe combined components of HBII and digitonin extraction buffers (both defined above) to maximize the detection of ExoS-HA in cellular fractions.) Cells were then processed, essentially as described by the digitonin extraction protocol. After the lysates were centrifuged at $16,000 \times \mathrm{g}$ for 15 mintues at $4^{\circ} \mathrm{C}$, the pellet (membrane fraction) was resuspended in $750 \mu \mathrm{l}$ of $\mathrm{HBI}$ buffer containing $0.1 \%$ Triton X-100. Protein concentrations of cytosolic and membrane fractions were determined using the BCA 
Protein Assay Kit (Pierce, Rockford, IL). Laemmli sample buffer was added to the remaining fraction volumes and samples were heated at $95^{\circ} \mathrm{C}$ for 5 minutes. Equal amounts of protein were resolved by SDS-PAGE, and localization of T3S-T-ExoS to cytosolic or membrane fractions was determined by immunoblot analysis using an antiHA antibody (Covance). To monitor protein loading, immunoblots were probed with an antibody against heat shock protein 70 , anti-HSP70, which is known to localize to mitochondrial, endoplasmic reticulum, nuclear and cytosolic fractions (BD Transduction Laboratories). The densities of protein bands were determined using Tiffany software (Humphrey Gardner, Scripps Institute, La Jolla, CA).

\section{Examination of T3S-T-ExoS intracellular processing}

\section{i) Effect of protease inhibitors on T3S-T-ExoS ADPRT activity}

To determine if T3S-T-ExoS is processed by a eukaryotic protease, eukaryotic cells were treated with diffusible protease inhibitors, and cell lysates were examined for ADPribosylation of eukaryotic proteins. In these studies, HL-60 cells were seeded and TPAdifferentiated as described in Chapter 3. One hour prior to co-culture with Pa strain 388, TPA-HL-60 cells were pretreated with a 1:500 dilution of a protease inhibitor cocktail (PIC), P8340, (Sigma), as well as, the appropriate concentrations of the individual protease inhibitor-components of the PIC. The 1:500 dilution of the PIC contains 208 $\mu \mathrm{M}$ AEBSF (4-(2-aminoethyl)benzenesulfonyl fluoride) (serine PI), $160 \mathrm{nM}$ aprotinin (serine $\mathrm{PI}$ ), $4 \mu \mathrm{M}$ leupeptin (serine and cysteine $\mathrm{PI}$ ), $8 \mu \mathrm{M}$ bestatin (aminopeptidase inhibitor), $3 \mu \mathrm{M}$ pepstatin A (acid PI), and $2.8 \mu \mathrm{M} \mathrm{E}-64$ (N-(trans-Epoxysuccinyl)-Lleucine 4-guanidinobutylamide) (cysteine PI). The 1:500 PIC dilution was found to have an effect on ExoS ADP-ribosylation, without affecting bacterial growth. Pretreatment of 
eukaryotic cells with PI solvents, including $\mathrm{dH}_{2} \mathrm{O}$ (AEBSF, aprotinin, leupeptin), DMSO

(PIC, bestatin, E-64) and 10\% acetic acid in methanol (pepstatin A), served as controls. After a 4 hour co-culture period with strain 388 or no bacteria, eukaryotic cells were lysed in Laemmli sample buffer, heated at $95^{\circ} \mathrm{C}$ for 5 minutes, resolved by SDS-PAGE and examined by immunoblot analysis, as previously described.

\section{ii) Effect of PIC on Pa T3S}

To determine if the PIC and AEBSF had an effect on $\mathrm{Pa}$ T3S, strains $388,388 \Delta \mathrm{S}$, $388 \triangle \mathrm{PopD}$, and 38811 were cultured with the drugs and then monitored for the production of PopD, a protein required for T3S-T. In these studies, bacteria from frozen stock were initially cultured overnight in LB, then cultured for an additional 14 hours in dialyzed tryptic soy broth supplemented with $5 \mathrm{mM} \mathrm{CaCl}_{2}$ (to suppress T3S). For the experiment, $208 \mu \mathrm{M}$ AEBSF, 1:500 dilution of PIC, and appropriate diluent controls ( $\mathrm{dH}_{2} \mathrm{O}$ and DMSO) were added to $3 \mathrm{ml}$ of dialyzed tryptic soy broth supplemented with 5 mM EGTA (to induce T3S). The medium was then inoculated with $10^{7}$ bacteria/ml and incubated for 4 hours in a $37^{\circ} \mathrm{C}$ shaking water bath. At the end of the culture period, 300 $\mu \mathrm{l}$ of the bacterial culture was removed and $100 \mu \mathrm{l}$ of $4 \mathrm{X}$ Laemmli sample buffer was added to the sample. The samples contained bacteria and secretion products. The samples were processed for and resolved by SDS-PAGE and immunoblotted with an anti-PopD antibody (Dara Frank, Medical College of Wisconsin).

\section{Co-immunoprecipitation of T3S-T-ExoS}

To understand a mechanism associated with differences in T3S-T-ExoS substrate specificity between rodent and human cell lines, a co-immunoprecipitation (Co-IP) procedure was developed, which would allow for the purification of T3S-T-ExoS and 
ExoS-associated proteins. In initial studies, Co-IP reactions were performed using ExoS-HA and anti-HA antibodies and protein A-sepharose beads to IP the protein complexes. However, several complications were associated with this approach: 1) significant background was caused by the heavy chain of IgG (which resolves similarly to ExoS by SDS-PAGE) and Protein A breakdown products, 2) the HA tag is a small epitope, limiting the specificity of the reaction, which made it difficult to identify unique T3S-T-ExoS interacting proteins. To address these problems, we were given an enzymatically inactive ExoS mutant constructed with a C-terminal triple FLAG tag (Sigma) by Joe Barbieri (Medical College of Wisconsin), which is encode on the pUCP plasmid and expressed by PA103AUT (PA103AUTpUCPExoS-R146K/E379D/E381D3XFLAG). The triple FLAG epitope is approximately 22 amino acids in length and includes two additional FLAG epitopes for recognition by the M2 anti-FLAG tag antibody (Sigma).

T24, human epithelial cells, and TCMK-1, mouse epithelial cells, were seeded in $100 \mathrm{~mm}$ dishes, as previously described, and grown to $70-95 \%$ confluencey. Eukaryotic cells were co-cultured with $10^{7}$ PA103 UUTpUCP or PA103AUTpUCPExoS-R146KE379D/E381D-3XFLAG for 4.5 hours. Cells were then washed twice with DPBS and detached with $0.25 \%$ trypsin-EDTA (HyClone, Logan, UT) for 10 minutes. Trypsinized cells were removed from the dishes, and dishes were washed an additional time with DPBS (to maximize harvest of eukaryotic cells). The trypsin was neutralized with the addition of $1 \mathrm{ml}$ fetal bovine serum (CellGro, Mediatech, Inc. Herdon, VA). Eukaryotic cells were pelleted by low-speed centrifugation and then resuspended in $800 \mu \mathrm{l}$ of HBII buffer (defined above). Cells were lysed by passage 20 times through a 27 gauge 
needle or by passage 15 times through a 28 gauge needle. Unbroken cells and nuclei were pelleted by centrifugation at $16,000 \times \mathrm{g}$ for 5 minutes at $4^{\circ} \mathrm{C}$. A $24 \mu \mathrm{l}$ volume of $5 \mathrm{M}$ $\mathrm{NaCl}$ was added to lysates to obtain a final concentration of $150 \mathrm{mM} \mathrm{NaCl}$. Protein concentrations in lysates were determined using a BCA Protein Assay (Pierce). Prior to the co-immunoprecipitation reaction, $30 \mu \mathrm{l}$ of anti-FLAG M2-Agarose affinity resin (Sigma) was washed 3 times in HBII, pelleting the resin by centrifugation at $8,200 \times \mathrm{g}$ for 30 seconds at $4^{\circ} \mathrm{C}$ between washes. Equal amounts of protein were added to the washed resin, and reactions were rotated at $4^{\circ} \mathrm{C}$ for 2 hours. Resin was washed 5 times in Tris buffered saline (TBS, $50 \mathrm{mM}$ Tris, $150 \mathrm{mM} \mathrm{NaCl}$, pH 7.4 supplemented with $1 \%$ EDTA and PIC) centrifuging at $8,200 \mathrm{xg}$ for 30 seconds at $4^{\circ} \mathrm{C}$ between washes. To elute the co-immunoprecipitate, resin was resuspended in $100 \mu$ of TBS containing 150 ng of $3 X$ FLAG peptide (Sigma) and rotated overnight at $4^{\circ} \mathrm{C}$. The supernatant containing the co-immunoprecipitate was obtained by pelleting the resin by centrifugation at $8,200 \times \mathrm{g}$ for 30 seconds at $4^{\circ} \mathrm{C}$. $4 \mathrm{X}$ Laemmli sample buffer was added to the supernatant and heated at $95^{\circ} \mathrm{C}$ for 5 minutes. An aliquot of the sample was analyzed by SDS-PAGE and immunoblot analysis with the M2 anti-FLAG antibody (Sigma). The remainder of the sample was resolved by SDS-10\% PAGE on an $13.0 \mathrm{~cm}$ X $14.5 \mathrm{~cm}$ gel, and protein was visualized by Imperial Protein Stain (Pierce), Gel Code Color Silver Stain Kit (Pierce) or SilverSNAP Stain for Mass Spectrometry (Pierce). Seven samples were excised from the gel and sent to James Carroll (Department of Molecular Genetics and Biochemistry, University of Pittsburgh School of Medicine, Pittsburgh, PA) for tryptic digestion, and MALDI-MS (matrix-assisted laser desorption/inonization mass spectrometry) analysis using an ABI 4700 TOF-TOF 
Proteomic analyzer (Applied Biosystems, Foster City, CA) located in the Genomics and Proteomics Core Laboratories at the University of Pittsburgh. As a negative control, a portion of the gel, which lacked discernible protein bands, was excised and processed. Results were analyzed using Prospector software, v 4.0.5 (Mass Spectrometry Facility, University of California at San Francisco, San Francisco, CA). 


\section{Results}

\section{Effects of MLD, GAP and ADPRT mutations on T3S-T-ExoS eukaryotic substrate modification}

ExoS is a biglutamic ADP-ribosyltransferase, with E379 conferring the transfer of the ADP-ribose to target proteins and E381 conferring the action of cleaving the ADP-

ribose from NAD molecules (Figure 1A) $(141,186)$. Previous in vitro analyses indicated that an E381 ExoS mutant would not be able to ADP-ribosylate substrates (141). To corroborate previous studies and determine which residues were required for in vitro ExoS ADPRT, equal amounts of supernatant from T3S-induced $\mathrm{Pa}$ cultures that expressed wild-type or mutant ExoS were incubated with lysates from eukaryotic cells for 1 hour at room temperature. Mutation of either E379 or E381 resulted in lack of ADP-ribosylation of RalA or Rac1 in both rodent (TCMK-1) and human (T24) cell lysates (Figure 1B). These results were consistent with the previously observed lack of ExoS ADP-ribosylation of Ras in vitro by E379A and/or E381A ExoS mutants $(64,186)$.

Previously it was shown that the eukaryotic cell influences which substrates T3S-TExoS targets $(65,197)$. To better understand how the host cell specifically influences T3S-T-ExoS restrictive or extensive patterns of ADP-ribosylation, rodent (TCMK-1 or Swiss-3T3) and human (T24) cells were co-cultured with Pa strain PA103 $\Delta$ UT which expressed a pUCP plasmid encoding a wild type or mutant-form of ExoS (Figure 1C). Interestingly, T3S-T-ExoS-E381A was able to modify both Ras and RalA within eukaryotic cells, albeit to a more limited extent than ExoS. However, it was unable to ADP-ribosylate cellular Rabs and Rac1 (Figure 1B and Figure 2). The E381A mutation did not alter how T3S-T-ExoS targets substrates within rodent cell lines. 
A. Linear model of ExoS

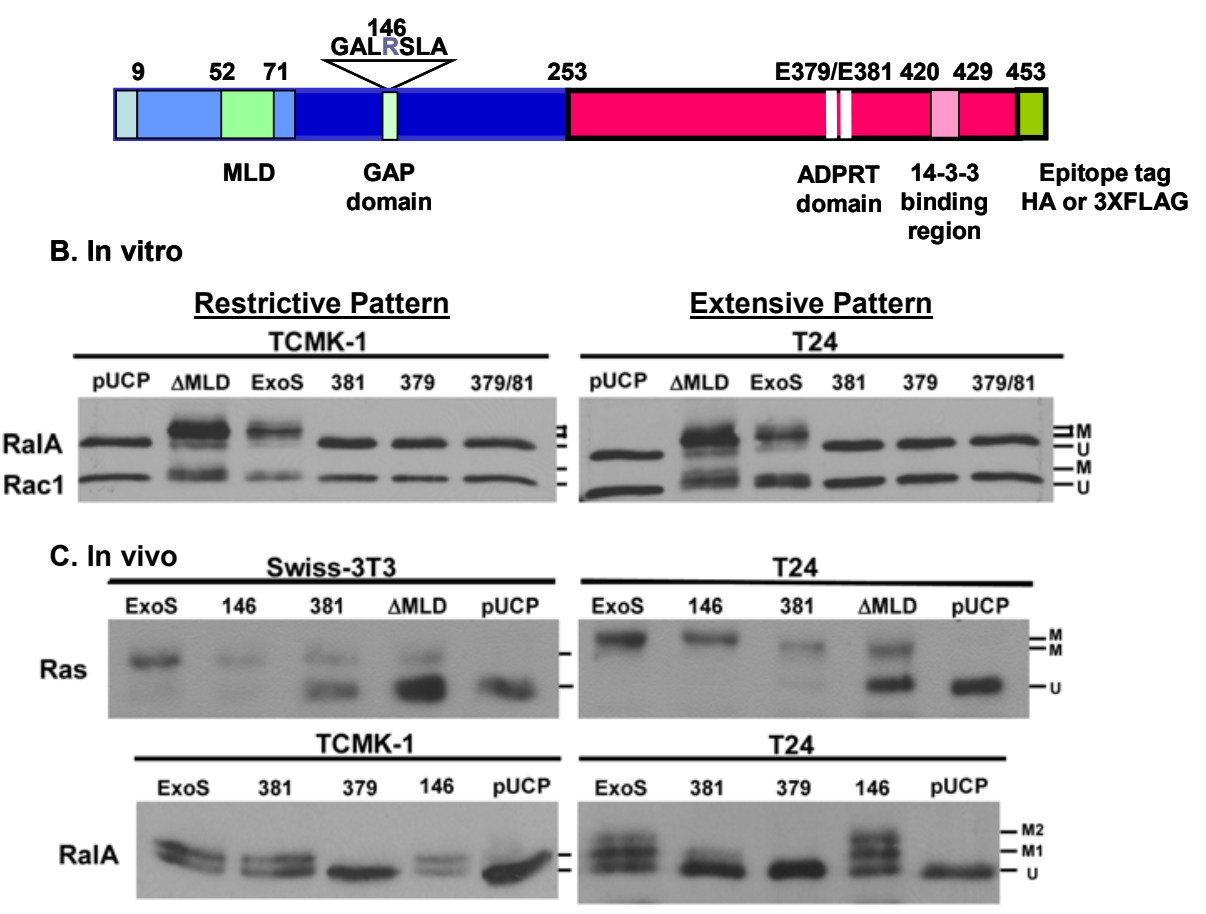

Figure 21. Effect of MLD, GAP and ADPRT mutations on ExoS ADP-ribosylation of cellular substrates.

A. Linear model of ExoS showing the location of the MLD (residues 52-71) (176), GAP domain (residues 72-253) (77), ADPRT domain (residues 253-453) (117, 141, 186 ), and the 14-3-3 binding domain (residues 420-429) (90). The location of the HA epitope tag (176) or the 3XFLAG epitope tag (J. Barbieri, personal communication) are also indicated. A detailed description of the linear map is included in Figure 6. B. In vitro analysis. Wild type or the indicated mutant ExoS, obtained from bacterial culture supernatants, was incubated with NAD and cell lysates for 1 hour at room temp, as previously described. Reactions were stopped with the addition of $4 \mathrm{X}$ Laemmli sample buffer, heated to $95^{\circ} \mathrm{C}$ for minutes, and resolved by SDS-PAGE. The indicated proteins were immunoblotted with specific antibodies and developed by ECL. C. In vivo analysis. Eukaryotic cells were co-cultured with bacteria expressing wild type or mutant ExoS, harvested, and examined as described in B. ADP-ribosylated (M) and unmodified (U) proteins are indicated. M1 and M2 refer to the first and second modifications of RalA by T3S-T-ExoS. Cell lines with restrictive and extensive T3S-T-ExoS ADPRT-modification patterns are indicated. 
Also evident in these studies was the lack of substrate modification by the E379A mutant, indicating the absolute requirement for the E379 residue for T3S-T-ExoS substrate ADP-ribosylation in vivo (Figure 21C).

It was clear that the E379A and E381A affected how ExoS ADPRT activity was targeted within the cell, however, the mechanism for these differences were not clear. To help understand the cellular mechanism associated with the effects of E381 and/or E379 mutations on T3S-T-ExoS substrate specificity, patterns of T3S-T-ExoS ADPribosylation or T3S-T-ExoS localization were monitored in cell lines exhibiting the restrictive (CHO, NIH-3T3, Swiss-3T3) or extensive pattern (Vero, T24, Wi38-SV40t). Cell fractionation was previously found to facilitate detection of subtle differences in ExoS ADPRT substrate ADP-ribosylation. For example, modification of Cdc42 was undetectable in whole HT-29 cell lysates, but became evident upon fractionation (65). In these studies, cells were fractionated by digitonin extraction after co-culture with $\mathrm{Pa}$ strains producing wild type or mutant ExoS constructs, and the resultant cytosolic and membrane fractions were examined for substrate modification of RalA, Rac1 and Rab5. 


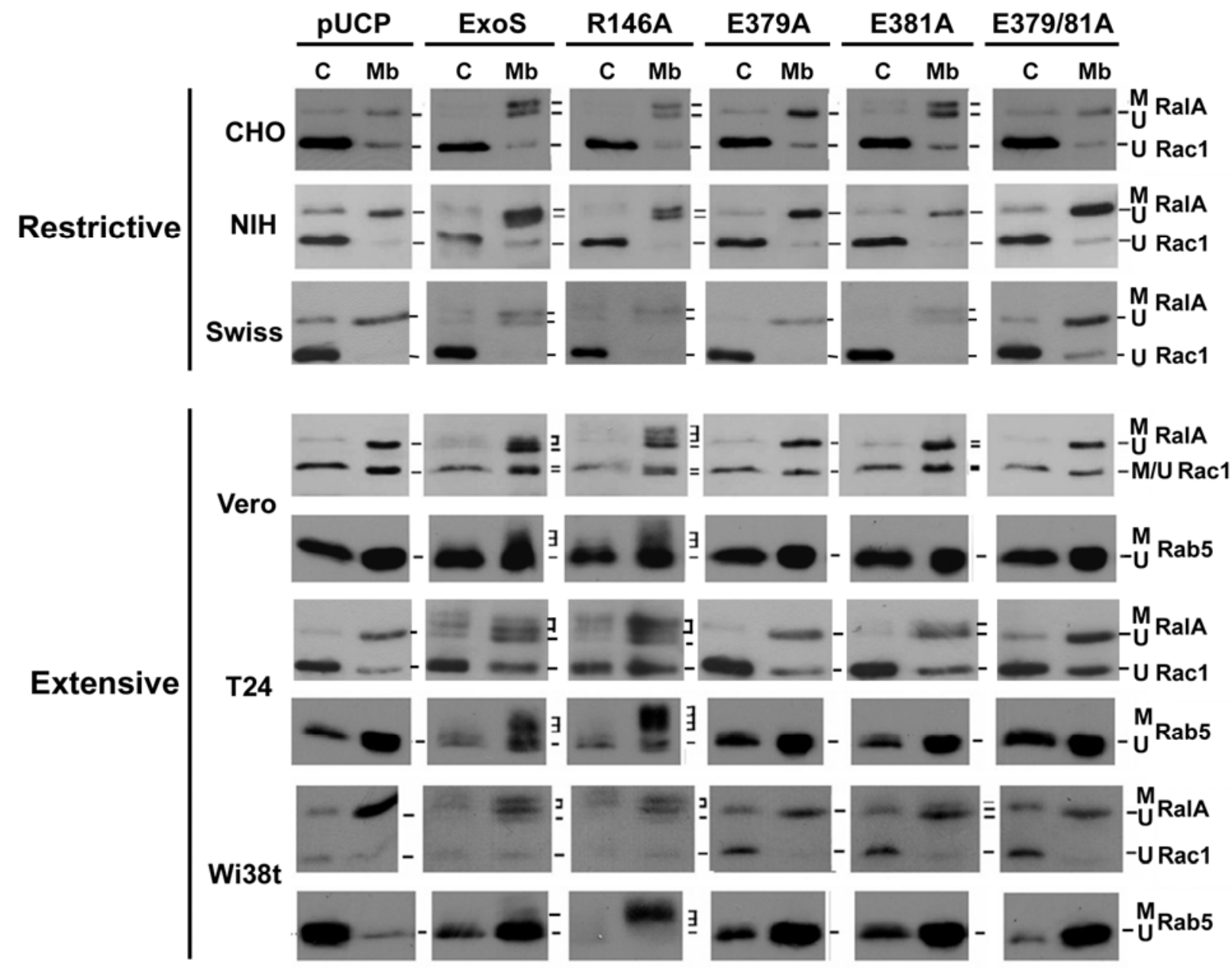

Figure 22. Digitonin extraction of eukaryotic cells treated with Pa expressing wild type or mutant ExoS.

After a 5 hour co-culture period with PA103 UUT expressing the indicated ExoS mutants or empty vector pUCP, eukaryotic cells were fractionated into cytosolic (C) and membrane $(\mathrm{Mb})$ fractions. Samples were resolved by SDS-PAGE, followed by Western blot of the indicated proteins, and visualized using ECL. Cells lines belonging to the extensive or restrictive pattern of substrate modification are indicated. Rab5 modification is shown only for cell lines having an extensive ADPRT substrate pattern (Vero, T24, and Wi38t). ADP-ribosylated (M) and unADP-ribosylated (U) proteins are indicated. 
As evident in Figure 22, RalA remains predominately in the membrane fraction, Rac1 remained predominately in the cytosolic fraction, and Rab5 was equally distributed between the two fractions in cells treated with PA103 UUTpUCP. Elimination of ExoS GAP activity by mutation of the arginine at position 146 to an alanine (ExoSR146A) did not affect ExoS substrate specificity when it was bacterially translocated into eukaryotic cells (Figure $21 \mathrm{C}$ and Figure 22). However, in cells with an extensive ADPRT substrate modification pattern, ADP-ribosylation of RalA and/or Rab5 was more efficient in association with T3S-T of ExoSR146A, as compared to T3S-T of wild type ExoS. Consistent with analysis of whole cell lysates, T3S-T-ExoSE381A resulted in a single modification of RalA in both rodent and human cell lines and E379 was absolutely required for T3S-T-ExoS ADP-ribosylation of substrates. Also, both the E379 and E381 residues were found to be required for T3S-T-ExoS ADP-ribosylation of Rab proteins and Rac1, and the more efficient ADP-ribosylation of Ras and RalA. These studies identified a role of the E381 residue in the extensive ADPRT substrate pattern observed in human and simian cell lines, but not for ADP-ribosylation in rodent cell lines. Differences observed in substrate modification by the R146A and E381A mutants in human and rodent cell lines further highlight eukaryotic cell influences on the targeting of T3S-T-ExoS ADPRT activity.

Recently, a MLD has been identified to reside within amino acids 51-72 of ExoS and it functions to target ExoS to eukaryotic membranes (176). ExoS ADPRT activity has been found mainly within the membrane fraction, indicating that intracellular localization is important to T3S-T-ExoS ADPRT activity $(64,188)$. With the availability of the PA103AUT strain to express the ExoS $\Delta$ MLD-HA mutant, we asked how the 
A. Substrate modification

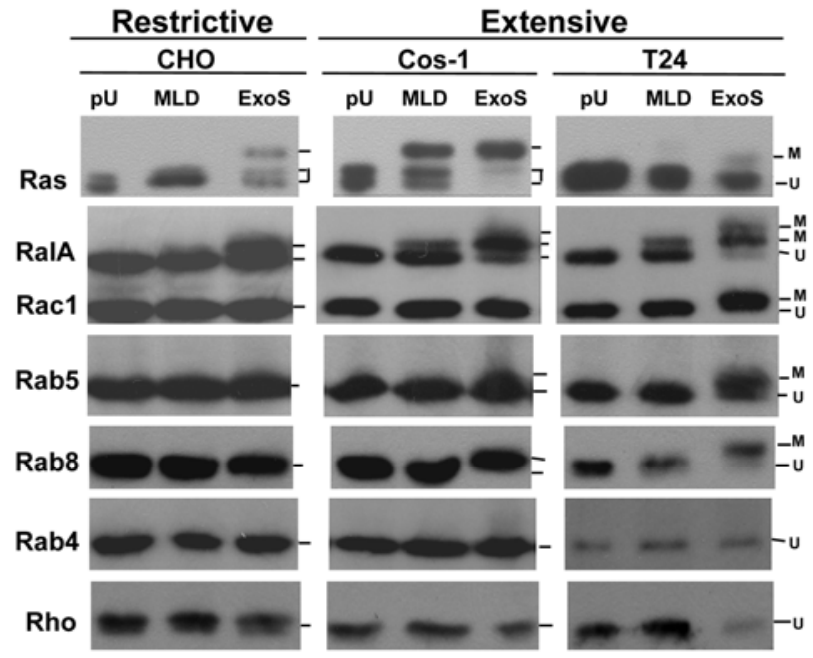

B. Intracellular localization

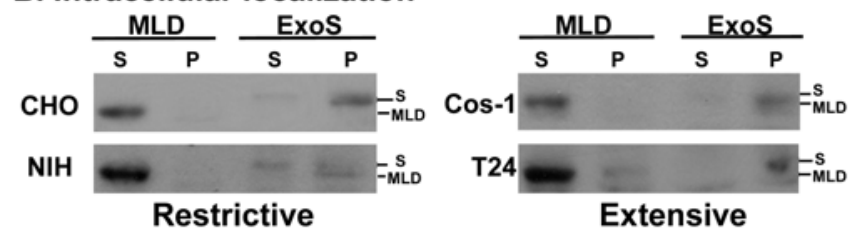

Figure 23. Effect of MLD on intracellular targeting and localization of T3S-T-ExoS ADPRT activity

A. Effect of MLD on T3S-T-ExoS substrate modification. Eukaryotic cells of restrictive or extensive patterns of substrate modification were co-cultured with PA103AUT expressing wild type ExoS-HA (ExoS) or ExoSAMLD-HA (MLD) or, pUCP $(\mathrm{pU})$ vector, for 4 to 5 hours, and harvested and examined for the indicated proteins by Western blot analysis, as previously described. ADP-ribosylated (M) and unmodified (U) proteins are indicated. B. Effect of MLD on T3S-T-ExOS intracellular localization. After co-culture with PA103 $\Delta$ UT expressing either ExoS $\triangle M L D-H A(M L D)$ or ExoS-HA (S), cell lines were harvested and fractionated by ultracentrifugation (refer to Methods and Materials), to produce cytosolic S100 (S) and membrane P100 (P) fractions. Fraction volumes were equilibrated and samples were resolved and immunoblotted using an anti-HA tag antibody, as previously described. ExoS (S) and ExoS $\triangle M L D$ proteins have different mobilities by SDS-PAGE, as indicated. The cytosolic ADPRT activity, which is measured in $\mathrm{fmol} / \mathrm{\mu g}$ min and corresponds to the data points displayed in Figure 3B, for 
T3S-T-ExoS $\triangle$ MLD-HA versus T3S-T-ExoS-HA are as follows: $\mathrm{CHO}, 116.305 \pm 5.8$ vs. $31.69 \pm 0.206$; $\mathrm{NIH}-3 \mathrm{~T} 3,61.78 \pm 3.3$ vs. $5.90 \pm 0.187$; Cos $-1,80.9 \pm 0.233$ vs. $17 \pm 0.28$; $\mathrm{T} 24,37.7 \pm 2.1$ vs. $1.68 \pm 0.07$. Restrictive pattern cell lines: $\mathrm{CHO}-\mathrm{K} 1(\mathrm{CHO}), \mathrm{NIH}-3 \mathrm{~T} 3$ (NIH); Extensive pattern cell lines: Cos-1, T24. 
MLD influenced the targeting of ExoS to eukaryotic substrates. In examining ExoS $\triangle M L D$ in an in vitro analysis, using T3S-induced $P$ a supernatant as a source of ExoS and ExoS $\triangle M L D$, and eukaryotic lysates as a source of 14-3-3 and substrates, ExoS $\triangle M L D-H A$ ADP-ribosylated proteins similarly to wild type ExoS (refer to Figure 21 A). When we compared the ADP-ribosylation of ExoS substrates in rodent $(\mathrm{CHO})$ or human (T24) cell lines after co-culture with $\mathrm{Pa}$ expressing ExoS-HA or ExoS $\triangle \mathrm{MLD}-\mathrm{HA}$ (Figure 21 and Figure $23 \mathrm{~A}$ ), T3S-T-ExoS $\triangle$ MLD-HA reduced the efficiency of ExoS ADPRT substrate modification. In rodent cells, Ras and RalA were modified by T3SExoS $\triangle M L D$, but much less efficiently than that observed in vitro (compare Swiss-3T3, Figure $21 \mathrm{C}$ and $\mathrm{CHO}$, Ras and RalA, Figure $23 \mathrm{~A}$ ). In T24 human epithelial cells, T3ST-ExoS $\triangle$ MLD-HA ADP-ribosylated substrates in a manner similar to that of wild type T3S-T-ExoS-HA in rodent cell lines. This was evident in the modification of Ras, a single modification of RalA, and no modification of Rabs 5 and 8 or Rac1 (Figure 21B and Figure $23 \mathrm{~A}$ ). In addition, T3S-T-ExoS $\triangle \mathrm{MLD}$-HA did not redirect ExoS to cellular targets, which are not normally ADP-ribosylated by T3S-T-ExoS-HA, such as Rab4 and Rho (Figure $23 \mathrm{~A}$ ). These results indicate that the MLD may play a greater role in targeting ExoS to eukaryotic proteins in human cells, than in rodent cells. 


\begin{tabular}{cccccc} 
& \multicolumn{3}{c}{ ExoS ADPr pattern } & \multicolumn{2}{c}{ Mutant ExoS ADPr } \\
Substrate & Intracellular & Extensive & Restrictive & E381A & $\Delta$ MLD \\
& localization & & & & \\
Ras & PM & $+^{*}$ & + & + & + \\
RalA & PM & $+^{*}$ & + & + & + \\
Rab5 & CCV, EE & + & - & - & - \\
Rab8 & tG, SG & + & - & - & - \\
Rab7 & LE & + & - & - & - \\
Rab11 & pnRE, pgRE & + & - & - & - \\
Rac1 & Cyto/PM & + & - & - & -
\end{tabular}

Table 3. Summary of ExoS ADP-ribosylation patterns and subcellular localization of target proteins

Summary of targets of ExoS ADP-ribosylation within the eukaryotic cell. Extensive (human and simian cell lines) and restrictive (rodent cell lines) patterns are defined. The effect of E381A and $\triangle M L D$ mutations on substrate targeting in human cells is shown. The E381A and the $\triangle M L D$ mutations do not affect T3S-T ExoS targeting of substrates in rodent cell lines. + or - indicates whether proteins are ADP-ribosylated or not by ExoS. ${ }^{*}$ indicates a protein is more efficiently modified in human and simian cell lines, as compared to rodent cell lines. ADPr, ADP-ribosylation; PM, plasma membrane; CCV, clathrin coated vesicles; early endosomes, EE; trans-Golgi region, tG; secretory granules, SG; late endosomes, LE; perinuclear recycling endosomes, pnRE; perigolgi recycling endosomes, pgRE; cytosol, Cyto. 
To further explore the mechanism for diminished ADP-ribosylation by the ExoS $\triangle M L D$ mutant within eukaryotic cells, cytosolic and membrane fractions were compared for the presence of ExoS $\triangle M L D-H A$ and ExoS-HA, by Western blot analysis (Figure $23 \mathrm{~B}$ ) and by quantification of ExoS ADPRT within cytosolic and membrane fractions. T3S-T-ExoS $\triangle$ MLD-HA localized to the cytosolic fraction, while wild type T3ST-ExoS-HA localized mainly to the membrane fraction of both rodent and human cell lines. These results correlated with the greater amount of ExoS ADPRT activity detected in cytosolic fractions of cells treated with PA103AUT-pUCPExoS $\triangle M L D-H A$, as compared to the cytosolic fractions of cells treated with PA103AUT-pUCPExoS-HA. Also evident from these results was that the decreased ADP-ribosylation of substrates by ExoS $\triangle M L D-H A$ did not relate to a defect in the T3S-T of ExoS $\triangle M L D-H A$. In general, greater total amounts of T3S-T-ExoS $\triangle$ MLD-HA were detected in total lysates of all cell

lines (data not shown); however, bacteria also secreted ExoS $\triangle M L D-H A$ more efficiently than ExoS-HA (data not shown), indicating that this domain may be important for T3S of ExoS, as well as, intracellular targeting of T3S-T-ExoS. Most importantly, these results indicate that without localization to the membrane fraction, T3S-T-ExoS can not efficiently ADP-ribosylate substrates.

\section{Examining intracellular processing of T3S-T-ExoS}

While examining the intracellular localization of ExoS, we often noticed multiple forms of ExoS in total lysates of cells treated with ExoS-HA producing bacteria (Figure 24 A). Although the heterogeneity in molecular mass and charge of ExoS had been noted previously (151), the function of these different forms of ExoS within eukaryotic cells remains unclear. In our studies we have observed three forms of T3S-T-ExoS by SDS-PAGE followed by Western blot analysis: a form which has a lower mobility than 
T3S-secreted ExoS, a form which has a similar mobility to T3S-secreted ExoS, a form which has a higher mobility than T3S-secreted ExoS (Figure $24 \mathrm{~A}$ and Figure 25). ExoS has been reported to have a processing site within the first 78 amino acids (177) and to ADP-ribosylate itself (auto-ADP-ribosylated) within the cell (189). Hence, we hypothesized that the lower mobility form of T3S-T-ExoS to be a processed form, and the higher mobility form to be the auto-ADP-ribosylated form. The processed form and the ADP-ribosylated form of T3S-T-ExoS have never been observed within the same sample. The ADP-ribosylated form is also more commonly observed than the processed form, suggesting that the lower mobility form may be an intermediate state or unstable form that precedes the self-ADP-ribosylated form of T3S-T-ExoS (personal observation). In addition, T3S-T-ExoS auto-ADP-ribosylation has been observed in both rodent and human cells, but was not observed when E379A and/or E381A mutants were bacterially translocated into the host cell, indicating that this event requires both glutamic acid residues (Figure 5).

To better understand the processed, intermediate form of T3S-T-ExoS, eukaryotic cells were treated with membrane permeable protease inhibitors and then co-cultured with ExoS producing bacteria. Initial results indicated that a 1:500 dilution of the protease inhibitor cocktail (PIC), eliminated ADP-ribosylation of Rab5 (Figure 24 B). The 1:500 dilution of the PIC did not have an effect on eukaryotic cell viability or bacterial cell growth (data not shown). In examining the individual components of the PIC, 208 $\mu \mathrm{M}$ AEBSF, which is a serine protease inhibitor, was the component which was 
A.
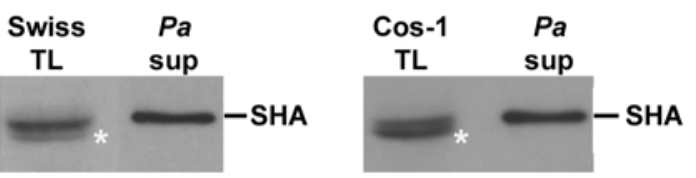

B.

TPA-HL-60 cells treated with $P$ a strain 388
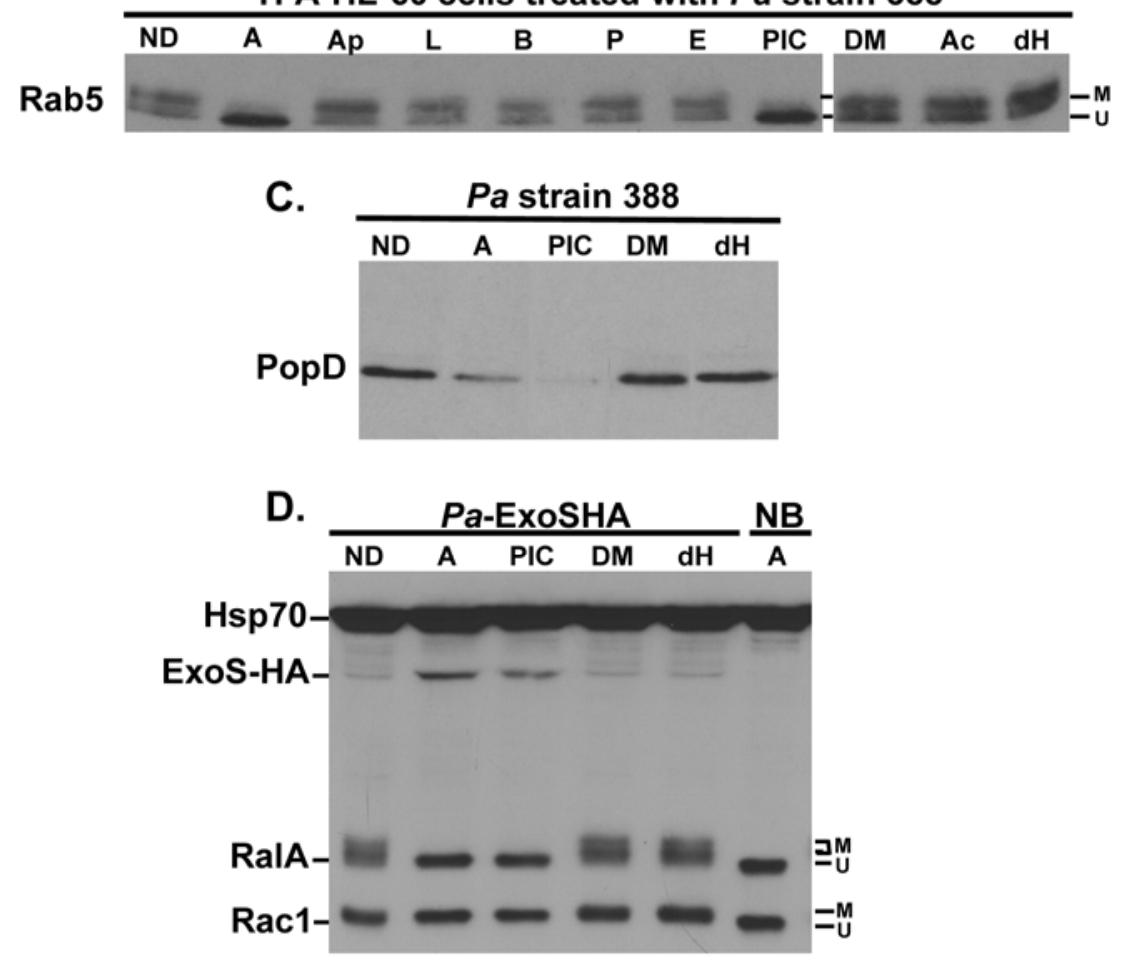

Figure 24. Effect of protease inhibitors on the intracellular targeting and T3S-T of ExoS ADPRT activity.

A. Cellular processing of T3S-T-ExOS. Eukaryotic cell lysates (TL) were prepared from the indicated cell lines and resolved by SDS-PAGE and immunoblotted for ExoS, using the anti-HA tag antibody. The asterisk denotes the lower, processed form of ExoS observed in TL but not in bacterial supernatants (Pa sup). B. Effect of components of the PIC on ExoS ADP-ribosylation of Rab5. TPA-HL-60 cells were treated with individual components of the PIC, prior to incubation with Pa strain 388. Samples were resolved by SDS-PAGE, followed by Western blot analysis for Rab5 and visualized by $E C L$. ADP-ribosylated (M) and unmodified $(U)$ forms of Rab5 are indicated. No drug, ND; AEBSF, A; Aprotinin, Ap; Leupeptin, L; Bestatin, B; Pepstatin A, P; E-64, E; 
Protease inhibitor cocktail, PIC; DMSO, DM; $10 \%$ Acetic acid, $\mathrm{Ac} ; \mathrm{dH}_{2} \mathrm{O}, \mathrm{dH}$. C. Effect of $P I C$ and AEBSF on production of PopD. Pa strain 388 was incubated with no drug, PIC, AEBSF or carrier controls for 4 hours, prior to the combined harvest of bacterial cells and bacterial supernatants. Samples were resolved by SDS-PAGE, followed by Coomasie staining or immunoblot analysis for PopD (shown). To ensure equal protein loading, densitometry analysis was performed on the Coomasie stained gel and raw densitometry values for total bacterial supernatant are as follows: ND, 24.56; A, 25.31; PIC, 26.28; DM, 27.73; dH, 28.04. D. Effect of AEBSF and PIC on T3S-Tof ExoS. After treatment with the indicated drugs or controls, T24 cells were trypsinized and processed for SDS-PAGE and immunoblot analysis. Membranes were incubated simultaneously with antibodies against heat shock protein 70 (HSP70), HA tag, RalA and Rac1, and visualized by ECL. ADP-ribosylated (M) and unmodified $(U)$ proteins are indicated. No bacteria, NB. 
responsible for the inhibition of ADP-ribosylation of Rab5 in TPA-differentiated HL-60 cells (Figure $24 \mathrm{~B}$ ). As a control, bacterial cells were cultured in the absence of eukaryotic cells and examined for production of a protein required for $\mathrm{Pa}$ T3S, PopD. Unexpectedly, AEBSF and the PIC, lessened bacterial production of PopD (Figure 24 C). These data suggested that the drugs affected the assembly/production of the bacterial T3S apparatus. Unexpectedly, T3S-T-ExoS was more evident in cells treated with either AEBSF or PIC, than control cells treated with carrier compounds or no drug. Despite greater amounts of T3S-T-ExoS, modification of RalA or Rac1 or ExoS autoADP-ribosylation was not evident (Figure 24 D). Also, despite a less robust production of PopD in AEBSF-treated Pa strains, there was enough PopD produced to allow T3ST. These results could indicate that only a minimal amount of PopD is required for T3ST or that another $\mathrm{Pa}$ T3S protein can substitute for function. Overall, these results indicate that AEBSF interfered with both Pa PopD production, and with T3S-T-ExoS ADPRT activity.

\section{Role of the R146 GAP residue in T3S-T of ExoS}

Seven ExoS-HA tagged mutants, which included single or combination mutations in the GAP (R146) and ADPRT (E379, E381) activities of ExoS, were constructed using the pUCP-ExoS-HA construct provided by Joe Barbieri (Medical College of Wisconsin, Milwaukee, $\mathrm{WI}$ ). These mutants allowed further exploration of mechanisms associated with their recognized differences in substrate specificity (refer to Figures $21 \mathrm{C}$ and 22), and also to examine the effects of the mutations on the T3S-T of ExoS. For these studies, eukaryotic cells were co-cultured with PA103AUT expressing wild-type or mutant forms of ExoS-HA. Following the co-culture, eukaryotic cells were harvested and fractionated into cytosolic and membrane fractions. 


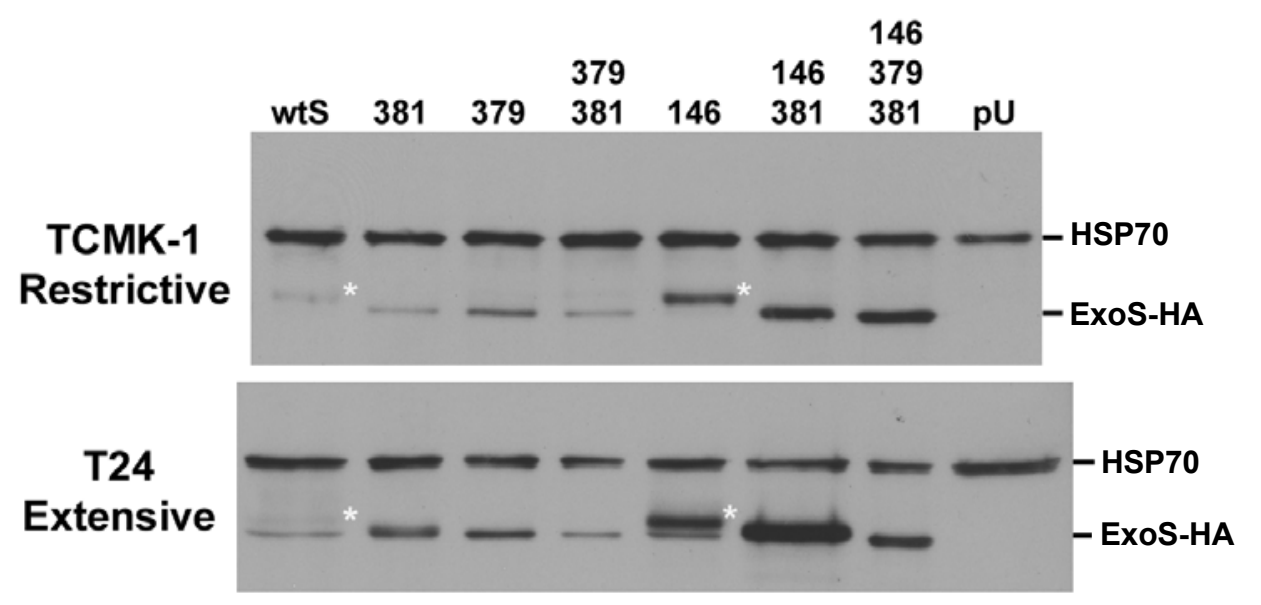

Figure 25. Effect of GAP and ADPRT mutations on the T3S-T of ExoS.

Membrane fractions were obtained by the digitonin extraction method (described in the Methods and Materials) from eukaryotic cells after co-culture with PA103 $\Delta$ UT expressing wild type ExoS (wtS), the indicated mutant ExoS, or empty vector control, pUCP $(p U)$. Samples were resolved by SDS-PAGE and immunoblotted for loading control, HSP70, and ExoS-HA. Auto-ADP-ribosylated forms of ExoS are indicated by an asterisk. 
T3S-T-ExoS was predominately found in the membrane fraction. In examining, the T3Stranslocation of ADPRT mutants, both E379 and E381 mutants were T3S-T into membrane fractions similarly to wild type ExoS in both rodent and human cell lines (Figure 25). These results indicated that the limited or lack of ADP-ribosylation of substrates by the E381A and E379A ExoS mutants, respectively, was not due to the lack of T3S-T. Interestingly, auto-ADP-ribosylated forms of ExoS were detected only for wild-type T3S-T-ExoS and T3S-T-ExoSR146A (asterisks, Figure 25). Mutation of $\mathrm{R} 146 \mathrm{~A}$, in the absence or in combination with E379A and/or E381A mutations, resulted in more efficient T3S-T of ExoS as compared to wild type ExoS (Figure 25). Results for the T3S-T of R146A/E379A were consistent with R146 contributing to increased T3S-T of ExoS (data not shown). These results support a role of the R146 residue in either regulation of T3S-T or in signals that mark T3S-T-ExoS for degradation. 

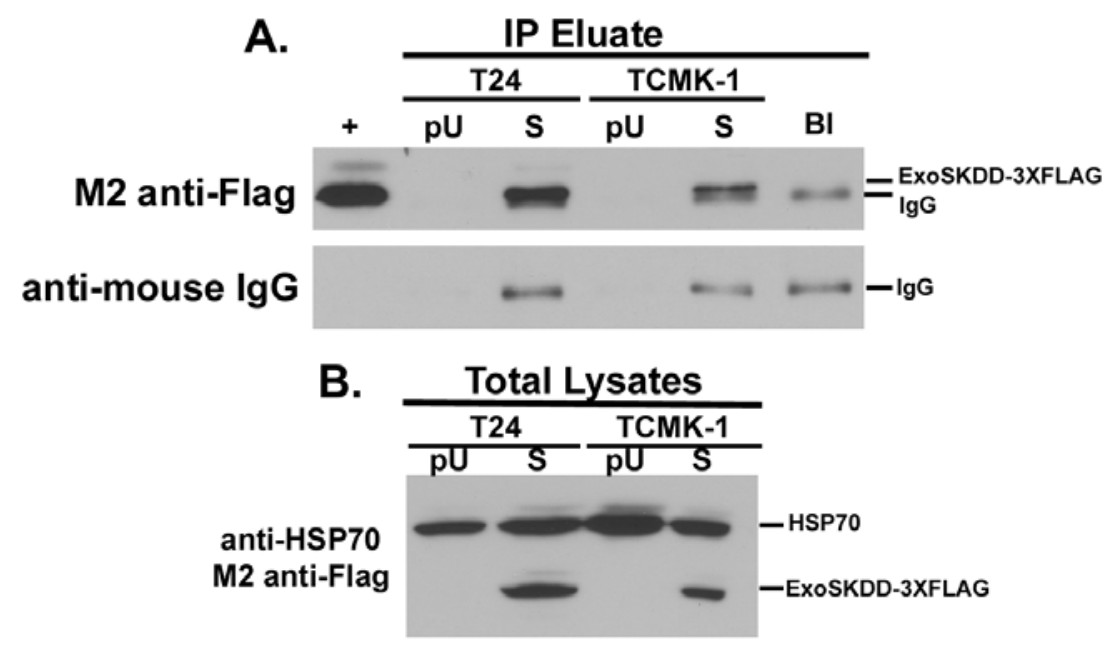

Figure 26. Immunoprecipitation of T3S-T-ExoS.

T24 (extensive pattern) or TCMK-1 (restrictive pattern) cells were co-cultured with PA103AUT expressing empty vector pUCP (pU) or pUCP-ExoS-R146K-E379/81D3XFLAG (S). A. Cells were harvested and T3S-T-ExoS was immunoprecipitated with M2 FLAG antibody immobilized to resin (Sigma) as described in the Methods and Materials. As a negative control, the IP procedure was performed using lysis buffer only (Blank, BI). Eluates from the IP reaction were resolved by SDS-PAGE, followed by Western blot analysis using the M2 FLAG mouse monoclonal antibody (Sigma) and anti-HSP70 antibody (BD Transduction Labs) (followed by anti-mouse lgG::HRP) or only, anti-mouse lgG, conjugated to HRP. B. Total lysates samples were resolved by SDS-PAGE and immunoblotted with M2 anti-FLAG tag (Sigma) and anti-HSP70 (BD Transduction Labs). 


\section{T3S-T-ExoS protein interactions within rodent and human cell lines}

An important objective of these studies was to identify a mechanism for the differential targeting of T3S-T-ExoS ADPRT activity in rodent and human cell lines. Initially we attempted to localize T3S-T-ExoS-HA within the eukaryotic cell by immunofluorescence and flow cytometry, but the high background precluded meaningful interpretations of results. We next performed co-immunoprecipitation (Co-IP) assays to identify proteins that interact with T3S-T-ExoS, as a means to understand how intracellular ExoS ADPRT activity is targeted.

Originally, Co-IP studies were attempted with ExoS-HA constructs, but there were difficulties in interpreting the results due to high background from immunoglobulin $\mathrm{G}$ ( $\operatorname{lgG}$ ) heavy and light chains, and protein A as well as limited T3S-T of wild type ExoSHA (refer to Figure 25). Given these limitations, we chose to use an enzymatically inactive construct of ExoS, with mutations in the GAP and ADPRT regions (R146K, E379D, E381D) that included a C-terminal triple FLAG tag (ExoS-KDD-3XFLAG) (gift from Joe Barbieri, Medical College of Wisconsin) for these studies. Mutation of R146 has been shown to facilitate T3S-T of ExoS (refer to Figure 25). The use of the $3 \mathrm{X}$ FLAG epitope in combination with the M2 FLAG antibody immobilized to resin for the Co-IP reaction, eliminated the background from Protein A and reduced background from IgG. However, using an enzymatically inactive form of ExoS, potentially compromised the detection of protein interactions directed by key catalytic residues in ExoS. 

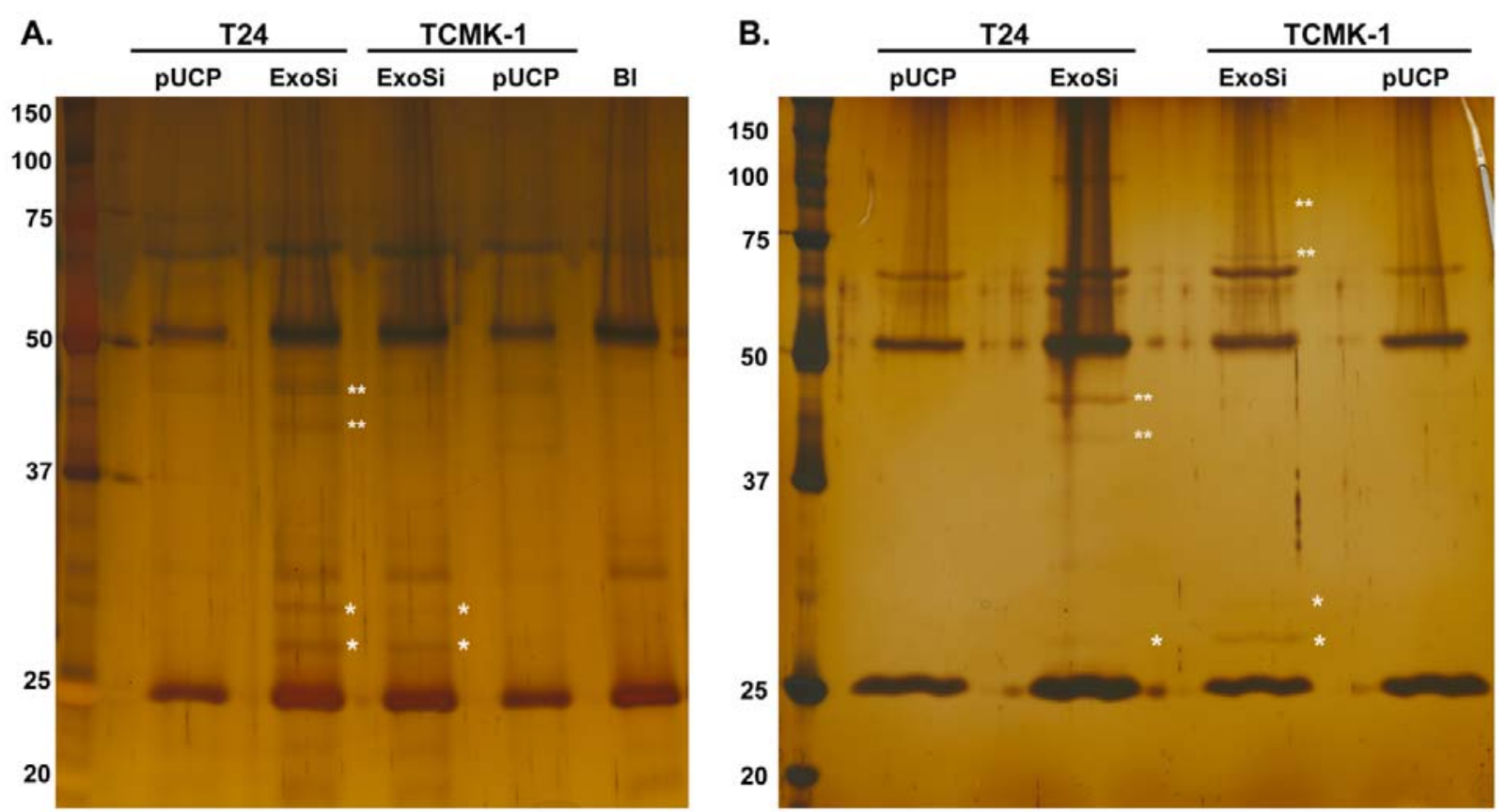

Figure 27. Silver stain analysis of T3S-T-ExoS IP eluates.

IP eluates were obtained from T24 (extensive pattern) and TCMK-1 (restrictive pattern) cells co-cultured with PA103AUT expressing pUCP or pUCP-ExoS-R146K-E379/81D3XFLAG (ExoSi). As a negative control (BI), lysis buffer alone was added to the M2 FLAG antibody-immobilized resin. Samples were resolved by SDS-10\% PAGE and gels were silver stained using A. the Gel Code Color Silver Stain Kit (Pierce) and B.

SilverSNAP Stain for Mass Spectrometry (Pierce). Molecular weights of standards are indicated. A single asterisk indicates proteins identified in IP eluates from both ExoSi treated cell lines. A double asterisk indicates proteins found in either T24 or TCMK-1 cells treated with ExoSi producing bacteria. All astericked proteins in B. were excised for MALDI-MS analysis. The SilverSNAP Stain for Mass Spectrometry was found to lightly stain gels, but unique proteins in T24-ExoSi (40 and $45 \mathrm{kDa}$ ) and TCMK-1-ExoSi (70 and $85 \mathrm{kDa}$ ) IP eluates were more easily identifiable. 


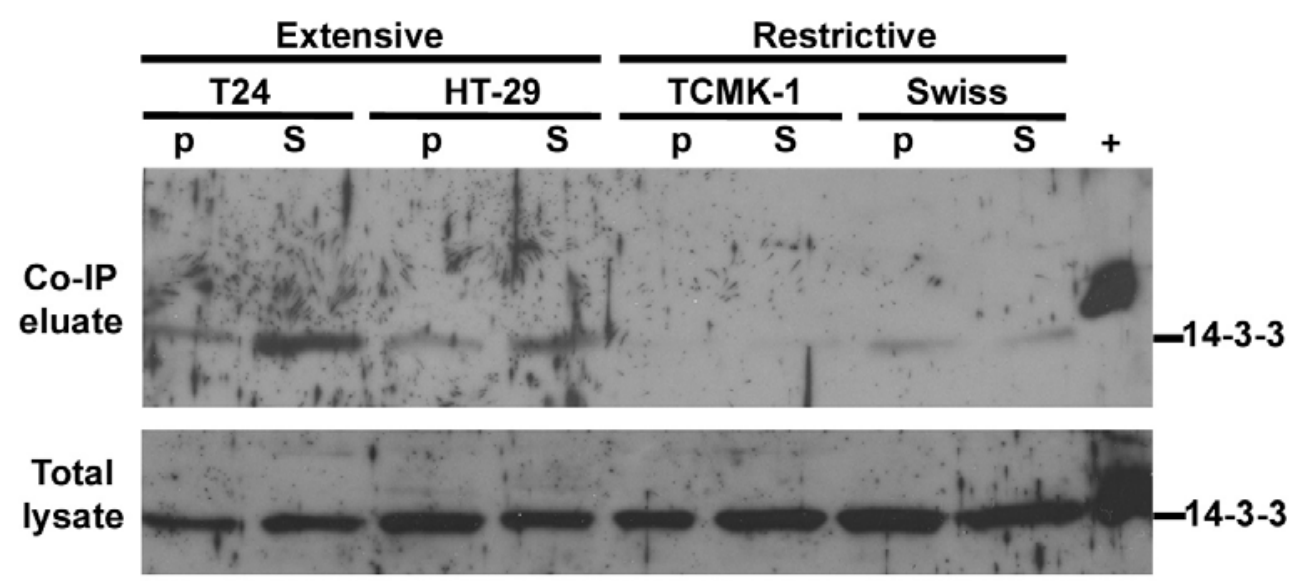

Figure 28. T3S-T ExoS co-immunoprecipitates with 14-3-3.

As previously described, total lysates and Co-IP eluates were obtained from T24 and HT-29 cells (extensive pattern), and TCMK-1 and Swiss-3T3 (Swiss) cells (restrictive pattern) after co-culture with PA103 UUT expressing empty vector pUCP (p) or pUCPExoS-R146K-E379/81D-3XFLAG (S). Samples were resolved by SDS-12\% PAGE, followed by Western blot analysis using an anti-14-3-3 rabbit polyclonal antibody (Upstate Biotechnology), which recognizes $\zeta$, $\gamma$, and $\eta$ 14-3-3 isoforms. 
We co-cultured rodent and human cell lines with PA103AUT expressing ExoS-KDD3XFLAG and T3S-T-ExoS and interacting proteins were extracted in a CoIP reaction. Samples were resolved by SDS-PAGE and either processed for Western blot analysis or silver stain analysis. Western blot analysis revealed that ExoS was successfully being immunoprecipitated from eukaryotic cells, albeit in the presence of a minimal IgG background (Figure 26). As a negative control, Co-IP eluates were immunoblotted for HSP70, which was not evident (data not shown). In comparison of Co-IP samples from rodent and human cell lines, two bands, which were approximately 27 and $30 \mathrm{kDa}$ in size, were present in both samples treated with ExoS-KDD-3XFLAG expressing bacteria (Figure 27). The $30 \mathrm{kDa}$ protein was identified by MALDI-MS to be the $\varepsilon$ isoform of the 14-3-3 protein family by matching 13 out of 214 peptides, with $50 \%$ coverage and a MOWSE score of 5.065e + 05 (J. Carroll, personal communication). The $27 \mathrm{kDa}$ protein was tentatively identified as a $14-3-3$ protein ( $\zeta, \mathrm{\gamma}$, or $\eta$ isoform) by Western blot analysis (Figure 28). The identification of $14-3-3$ proteins in Co-IP eluates from cells treated with ExoS-KDD-3XFLAG expressing bacteria served as a positive control. Additionally it is currently unknown which 14-3-3 isoform(s) interact with T3S-TExoS. ExoS also purified with $40 \mathrm{kDa}$ and a $45 \mathrm{kDa}$ human protein, and a $70 \mathrm{kDa}$ and an $85 \mathrm{kDa}$ rodent protein. The identification of different T3S-T-ExoS interactive proteins in rodent and human cells supports the hypothesis that the differential targeting of T3ST-ExoS ADPRT activity within human and rodent cell lines is due to differential protein interactions. 


\section{Discussion}

To further explore the mechanism underlying the differential targeting of T3S-TExoS to substrates in rodent and human cell lines (197), we designed studies to: 1) further characterize the differences in substrate specificity, and 2) identify mechanisms responsible for the differences in substrate specificity. All of these studies were based on the perspective that the host cell contributes to $\mathrm{Pa}$ T3S process. An example of host cell influence on the targeting of ExoS ADPRT activity is in the comparison of the repertoire of proteins that ExoS ADP-ribosylated in rodent cell extracts in vitro, including Ras, RalA, Rab proteins and Rac1, to the limited proteins that T3S-T-ExoS ADPribosylated in the cell, including only Ras and RalA (197).

Analysis of substrates targeted by wild type or mutant ExoS in vitro revealed that mutation of either glutamic acid, 379 or 381 , resulted in a lack of substrate modification and lack of auto-ADP-ribosylation of ExoS (personal observation) in both rodent and human cell extracts. These results confirmed previous in vitro studies, which characterized E381 as the critical residue for ExoS ADPRT activity (141). Unexpectedly, T3S-T-ExoSE381A ADP-ribosylated Ras and RalA in both rodent and human cell lines, but did not target Rab proteins or Rac1 in human cells. An auto-ADP-ribosylated form of ExoS-E381A-HA also was not observed in either rodent or human cell lines. These results indicated that the ExoS-E381A mutant exhibited ADPRT activity only after T3S-T into eukaryotic cells. These results were consistent with previous studies that indicated that the host cell could limit and influence the molecular targeting of T3S-T-ExoS ADPRT activity $(65,193,197)$, which led to the examination of the influence of 
intracellular localization and protein interactions on the targeting of ExoS ADPRT activity in rodent and human cells.

It was observed that ExoS ADPRT activity is found mainly in the membrane fraction of eukaryotic cells $(64,188)$. Concordantly, a membrane localization domain (MLD) was found to reside within residues 51-72 of ExoS and to localize T3S-T-ExoS to eukaryotic membranes. Loss of the MLD caused T3S-T-ExoS to localize to the cytosolic fraction of eukaryotic cells (176). The restrictive pattern of substrate modification was observed in human cells following T3S-T of ExoS $\triangle M L D$, and there was a modest decrease in the ability of T3S-T-ExoS to ADP-ribosylate proteins in rodent cells, which intrinsically exhibit the restrictive pattern of substrate modification. The ability of ExoS $\triangle M L D$ to ADP-ribosylate target proteins was similar to results observed upon T3S-T of the ExoSE381A mutant, which resulted in a restrictive substrate modification pattern in human cells, but did not alter the substrate modification pattern observed in rodent cells. The decreased ability of ExoS $\triangle M L D$ to ADP-ribosylate cellular proteins was linked to the inappropriate localization of ExoS to the cytosol. In contrast, T3S-T-ExoS-E381A localized to membrane fractions similarly to wild type ExoS in both rodent and human cell lines. These data indicated that inappropriate intracellular localization or handicapped ADPRT activity can lead to similar restrictive patterns of substrate modification, which suggests that there may be more than one mechanism affecting the intracellular targeting of ExoS ADPRT activity.

As mentioned previously, the functional role of T3S-T-ExoS auto-ADP-ribosylation, observed both in vitro and within the cell, has not yet been described (189). Auto-ADPribosylation by other bacterial ADPRT toxins, such as $E$. coli heat labile enterotoxin 
(LTA), has been noted to occur in in vitro reactions, but not within the cell and seemingly without any functional consequence to the toxin (223). In contrast, studies examining mammalian ADPRT's have found that auto-ADP-ribosylation affects enzyme function. For example, auto-ADP-ribosylation of ADP-ribosyltransferase 5 (ART5) was shown to decrease NADase activity, while increasing ADPRT activity of the molecule, implicating a role of auto-ADP-ribosylation in the regulation of the enzyme's function (244). ExoS has been shown to closely resemble mammalian ADP-ribosyltransferases, as evident in: 1). the sequence homology between the catalytic domain of ExoS and domains of vertebrate ADPRTs, 2) both ExoS and eukaryotic ADPRT's ADP-ribosylate cellular proteins at more than one site, and 3). ExoS, like eukaryotic ADPRTs, targets multiple proteins (72). These similarities suggest that ExoS auto-ADP-ribosylation may regulate ExoS ADPRT activity.

Initial studies indicated that the purpose of ExoS auto-ADP-ribosylation was to down regulate ExoS GAP activity by auto-ADP-ribosylating the R146 residue (189). However, the GAP activity of ExoS was hard to discern in non-macrophage, mammalian cell lines $(64,192,193)$, and the recorded effect of ExoS auto-ADP-ribosylation of the GAP region was unimpressive (189). In these studies, auto-ADP-ribosylation was observed to occur with ExoS-R146A GAP mutant, confirming that the R146 residue was not the only potential arginine target of auto-ADP-ribosylation. ExoS auto-ADP-ribosylation was observed following the T3S-T of wild type or R146A mutant ExoS in rodent and human cells, and appeared to require both E379 and E381 residues. Linking these results to previous observations about the role of E381 in the intracellular targeting of ExoS ADPRT activity indicate that the E381 residue of ExoS may contribute to ExoS auto- 
ADP-ribosylation in all cells lines, the extensive pattern of ExoS substrate modification in human and simian cell lines, but not to ExoS substrate ADP-ribosylation in rodent cell lines. Therefore, upon T3S-T of ExoS into rodent cells, the activity of E381 may be limited to auto-ADP-ribosylation only, without influencing ExoS substrate ADPribosylation.

The observation that the T3S-T-ExoS-R146A mutant seemingly ADP-ribosylated substrates more efficiently than wild type T3S-T-ExoS, prompted studies which examined the T3S-T of ExoS-R146A mutants into eukaryotic host cells. These studies found that mutation of R146 (alone or in combination with ADPRT mutations) resulted in increased T3S-T of ExoS into both restrictive and extensive pattern cell lines. These data indicated that ExoS GAP activity may play a role in regulation of bacterial translocation or overall stability of the protein. Previous studies, which explored the T3S-T of YopE, found that the GAP activity of YopE (the GAP domain of YopE has sequence homology to that of ExoS (259)) acted in part to prevent actin polymerization, by downregulating RhoGTPase activity, to prevent the T3S apparatus from forming pores in the eukaryotic cell membrane (240). This was evident in that mutation of the GAP activity in YopE resulted in cell lysis by the pores formed by the T3S-apparatus. In our studies, mutation of the GAP activity did not result in cell lysis or death (data not shown). However, we wished to determine if the integrity of the eukaryotic cell cytoskeleton influenced the T3S-T of ExoS. To examine if the GAP region of ExoS functioned similarly to YopE by altering the status of the cytoskeleton, we treated T24, human epithelial, cells with cytochalasin $D$, which inhibited actin polymerization, and colchicine A, which inhibited microtubule polymerization, prior to co-culture with ExoS- 
HA or ExoS146-HA bacteria. Interestingly, both drugs influenced the T3S-T of wild type ExoS-HA, but not ExoS-R146A-HA. These results indicate that potentially both actin and microtubule components play a role in the T3S-T of ExoS. These results also suggest that the GAP activity controls the amount of ExoS which is T3S-T into eukaryotic cells, independently of the cytoskeleton. However, these results do not rule out the possibility that proper intracellular degradation of T3S-T-ExoS might rely on a functional actin or microtubule cytoskeleton.

Results obtained by examining the T3S-T of both the R146A mutant and E381A mutant highlighted areas of the T3S-T process about which very little is known, but in which the host cell likely plays a role. These include: 1) the post T3S-translocation processing/folding procedure that must occur to form a functional T3S effector, 2) T3ST-ExoS turnover, and 3) intracellular trafficking of T3S-T-ExoS. Given the 2-3 nm diameter of the needle apparatus $(18,76,94,110)$, it has been hypothesized that all T3S-effectors remain in a partially unfolded state to be translocated through the T3Sapparatus (220). This necessitates that the final folding of the T3S-effector occurs within the eukaryotic host cell.

It has been hypothesized that ExoS is processed within the eukaryotic cell upon T3S-T (177), and what appears to be a processed form of T3S-T-ExoS has been observed in our studies. To better understand this process, we pretreated eukaryotic cells with cell permeable protease inhibitors prior to co-culture with ExoS producing bacteria. These studies identified the general serine protease inhibitor, AEBSF, as a drug that negatively affected production of PopD, yet seemingly enhanced the T3S-T of ExoS, but inhibited ExoS ADPRT activity. While these studies are curious, it is unclear if 
AEBSF inhibits transcription, translation or secretion of PopD. It is unlikely that AEBSF acts as a global $\mathrm{Pa}$ T3S inhibitor, given that ExoS is produced and bacterially translocated. This data supports the notion that the T3S-apparatus is formed after treatment with AEBSF; however, the composition of the apparatus may be altered. Previous studies found that in addition to PopD, PcrV and PopB were required for $\mathrm{Pa}$ T3S-T, and that deletion of any one of the proteins inhibited T3S-T $(67,80,81,208$, 235). The stability and solubility of PopD and PopB required a chaperone protein, $\mathrm{PcrH}$ (210). Deletion of PcrH also resulted in inhibition of T3S-T (21). Since T3S-T of ExoS was still observed in cells treated with AEBSF, it is unlikely that the drug caused complete depletion of PopB, PcrV or PcrH. However it remains unknown if AEBSF affects the production of these proteins in a manner that would deregulate T3S-T of effectors. These studies were limited by the lack of examination of how AEBSF affected the T3S-T of other Pa effectors.

The negative effect of AEBSF on T3S-T-ExoS ADPRT activity suggested that AEBSF interfered with the serine protease responsible for T3S-T-ExoS processing. AEBSF is a general serine protease inhibitor, which has been found to act as an inhibitor of eukaryotic cell differentiation (14), a caspase inhibitor (42), a tumor angiogenesis inhibitor (184), and lysosome associated protein cleavage disregulator (39). Therefore, the negative effects of AEBSF on T3S-T-ExoS ADPRT activity may be due to a wide-range of indirect effects, unrelated to T3S-T-ExoS intracellular processing. Interestingly, aprotinin and leupeptin, which are components of the PIC and serine protease inhibitors, did not affect T3S-T-ExoS translocation or ADPRT activity, indicating that there may be specificity in the action of AEBSF. 
Critical to understanding how the host cell modulates T3S-T-ExoS ADPRT activity is gaining insight into how T3S-T-ExoS is trafficked within rodent and human cell lines. Previous studies found that T3S-T-ExoS ADP-ribosylated Rab proteins in a distinct order (65), providing a clue that T3S-T-ExoS may be trafficked via vesicles in human cells. Attempts at tracking T3S-T-ExoS by both immunofluorescence and flow cytometry did not yield meaningful data due to the high amounts of background staining in both assays. Subcellular fractionation of cells treated with ExoS-HA-producing bacteria has not been attempted. While these studies would be beneficial to understanding the intracellular localization of T3S-T-ExoS, very little wild type ExoS-HA could be visualized in cells fractionated into cytosolic and membrane fractions, indicating a potential difficulty in identifying T3S-T-ExoS within specific cellular compartments. Therefore, a proteomics approach was designed, which involved co-culturing eukaryotic cells with ExoS-producing bacteria and then co-immunoprecipitating ExoS with interacting proteins. The identification of T3S-T-ExoS-interacting proteins was hypothesized to lead to the identification of the intracellular location of ExoS. In comparisons of co-immunoprecipitates from human and rodent cell lines, different T3ST-ExoS-interacting proteins were isolated in both human and rodent cell lines. Preliminary results from MALDI-TOF indicate that the human ExoS-interacting proteins may be involved in lysosomal trafficking (J. Carroll, personal communication). These results support the hypothesis that T3S-T-ExoS ADP-ribosylates distinct eukaryotic proteins in a restrictive and extensive pattern cell lines, in part, because of differences in interacting proteins or intracellular location. 
Two similar proteins co-immunoprecipitated with T3S-T-ExoS from rodent and human cells, and are thought to be 14-3-3 isoforms (J. Carroll, personal communication). There are seven different isoforms of 14-3-3 found in mammalian cells: $\zeta, \varepsilon, \gamma, \beta, \sigma, \eta, \tau(1)$; all of which are capable of stimulating ExoS ADPRT activity in vitro (268). Little is known about which 14-3-3, isoform interacts with T3S-T-ExoS within cells. Since, 14-3-3 proteins regulate subcellular localization, stability and molecular activity of their target proteins (46), characterizing the interaction of 14-3-3 with T3S-TExoS may be the key to understanding the intracellular targeting of ExoS ADPRT activity. Comparisons of the amino acid sequences of Homo sapiens and Mus musculus 14-3-3 isoforms, found the $\zeta$ and $\varepsilon$ isoforms to be identical, while all other isoforms displayed differences in one or more amino acids (personal observation). Differences in conserved proteins among mammals have previously been found to affect the outcome of infectious agents. One such example is the single residue difference between the human and mouse E-cadherin, which resulted in an abrogated infectivity of Listeria in mice (132).

Further analysis of Co-IP samples by MALDI-TOF should reveal potential differences in T3S-T-ExoS-14-3-3 interactions as well as positive identification of the unique proteins which interact with T3S-T-ExoS in rodent or human cell lines. It is currently unclear if ExoS interacting with different proteins in rodent and human cells would have a direct affect on the intracellular targeting of ExoS ADPRT activity, but the identification of ExoS-interacting proteins is expected to reveal the intracellular location of ExoS in rodent and human cell lines. Defining the differences in how T3S-T-ExoS ADPRT activity is targeted within rodent and human cell lines will provide a better 
understanding of how the host cell influences the T3S-T process and directs effector activity. 
Chapter 5: Discussion 
The bacterial T3S system allows the microbe to hijack host cell function to its advantage. In general, discussions and studies about T3S focus on the bacterial side of the process. Many elegant studies have shown that bacterial T3S effector proteins ingeniously mimic the function of eukaryotic proteins to manipulate the host cell (221). Currently, there is very little known about the eukaryotic cell contribution to the T3S process. Given the intimate nature of the bacterial-eukaryotic cell association required for T3S to occur, it is bold to assume that the eukaryotic cell is a passive participant in this process. However, in most studies, the role of the host cell in the T3S process is eclipsed by the overt pathogenicity of most Gram-negative organisms. An advantage to studying $\mathrm{Pa}$ is that this organism will only cause disease if the host is compromised. By nature, $\mathrm{Pa}$ colonization and infection of the host is a negotiation rather than a hostile takeover. Early studies of $\mathrm{Pa}$ T3S revealed that subconfluent monolayers of eukaryotic cells were sensitive to Pa T3S-T of ExoS, while confluent cells were less sensitive (58, $115,154)$. These were the first studies indicating that the status of the host cell influences T3S, corresponding to the opportunistic nature of $P a$ infections.

In 1978, ExoS was characterized as a Pa ADPRT toxin distinct from exotoxin A (ETA) (106). In mouse models of infection, ExoS was noted to contribute to $\mathrm{Pa}$ dissemination $(162,163)$, but curiously, ExoS was nontoxic by itself to tissue culture cells and animals $(25,162,170)$. The inability to assign a cellular effect to the ExoS prompted genetic analysis of its regulation and transcription. These studies led to the recognition that $P$ a harbors a T3S system, homologous to the Yersinia T3S system $(258,260)$. There are four known Pa T3S effector proteins (ExoS, ExoT, ExoU and ExoY), but ExoS is the best characterized effector. 
ExoS is a bifunctional protein and serves both as a GAP targeting Rho, Rac and Cdc42 in vitro (77), and an ADPRT targeting mainly low molecular weight G-proteins in vitro $(30,63,65)$. The broad range of target substrates of ExoS ADPRT activity differentiates ExoS from other bacterial ADP-ribosyltransferase toxins, which are highly specific. Transfer of an ADP-ribose moiety to a target protein typically alters or destroys the function of that protein. For example, ETA ADP-ribosylation of elongation factor-2 (EF-2) inhibits protein synthesis in the eukaryotic cell (105). Endogenous eukaryotic ADPRTs have also been identified that regulate cellular processes. Coordinate to a eukaryotic ADPRT, a cognate ADP-ribose hydrolase may reverse the addition of ADPribose moieties to target proteins. For instance, polymerization of a muscle cell cytoskeletal component, desmin, has been found to be controlled in part by ADPribosylation. ADP-ribosylation of desmin results in the inability of desmin to form filaments, and the cognate ADP-ribosyl hydrolase allows desmin to re-form filaments $(100,101)$. EF-2, which is a target of Pa ETA and diphtheria toxin (DT) ADPRT activity $(31,105)$, has been found to be ADP-ribosylated by endogenous eukaryotic ADPRTs $(35,53)$. While this reaction has not been fully characterized, the eukaryotic ADPRT was found to ADP-ribosylate a subset of total cellular EF-2 and the ADPRT reaction was found to be reversible (53). These studies suggest that bacterial toxins mimic the actions of eukaryotic ADP-ribosyltransferases, but the lack of a cognate ADP-ribose hydrolase that renders the bacterial ADP-ribosylation permanent and consequently detrimental to eukaryotic cell function.

In studies characterizing the functional effects of T3S-T-ExoS, the ADPRT activity of ExoS was found to be required for ExoS-specific inhibition of DNA synthesis, permanent 
cell rounding, and inhibition of adherence processes (64). Notably, the effects of ExoS on cell function have not thus far been linked to the ADP-ribosylation of a particular substrate. Current theories suggest that T3S-T-ExoS effectively inactivates host cell function by the collective ADP-ribosylation of eukaryotic proteins. While ExoS targets multiple proteins, recent studies have found that the eukaryotic cell environment limits the substrates targeted by T3S-T-ExoS ADPRT activity $(65,89,193,197)$. Previous studies in our laboratory using the HT-29 human epithelial cell line, identified Ras, RalA, Rabs 5, 7, 8, 11, Rac1 and Cdc42 as targets of T3S-T-ExoS $(63,65)$. Importantly, Rab4 or Rap proteins were identified as targets of ExoS ADPRT in vitro, but not within eukaryotic cells $(30,63,65)$. In time-course co-culture studies, T3S-T-ExoS was found to ADP-ribosylate proteins in a specific order; Ras, RalA and Rab5 were targeted first, followed by ADP-ribosylation of Rabs 8 and 11, and lastly, Rab7 and Rac1 (65).

Combined, these studies indicated that the host cell limits and specifies the substrates targeted by T3S-T-ExoS ADPRT activity.

During studies of the cellular effects of ExoS ADPRT, further understanding of how the host cell contributes to and influences T3S-T-ExoS ADPRT activity began emerging. The goal of my studies was to characterize the role of the host cell in the Pa T3S-T of ExoS. Specifically, these studies have: 1) shown that cell origin influences T3S-T-ExoS ADPRT substrate specificity, 2) determined that the host cell influences $\mathrm{Pa}$ T3S at the level of membrane translocation, and 3) provided evidence that T3S-T-ExoS protein interactions might influence ExoS ADPRT intracellular trafficking.

In studying the effects of T3S-T-ExoS on rodent fibroblasts and human epithelial cell function, it was found that the rodent fibroblasts were typically less sensitive than 
human epithelial cells to the effects of ExoS on DNA synthesis, permanent morphological alterations and readherence. These results correlate with the identification of two patterns of T3S-T-ExoS ADP-ribosylation of substrates: 1) a restrictive pattern, showing a single modification of Ras and RalA;2) an extensive pattern, recognized as more efficient modification of Ras and RalA and modification of Rabs 5, 7, 8, 11 and Rac1. The pattern of T3S-T-ExoS substrate modification was not linked to cell type or efficiency of T3S-T of ExoS, but to the species of origin, with rodent cell lines exhibiting the restrictive pattern of ADP-ribosylation, and simian and human cell lines exhibiting the extensive pattern of ADP-ribosylation (197).

Previous studies identified another instance in which human and rodent cell lines displayed differential sensitivities to a bacterial ADP-ribosyltransferase. Studies which examined cellular sensitivity to DT found that cells of monkey origin were the most sensitive to DT, followed by human and hamster cells, while cells of mouse and rat origin being resistant (157). Further examination showed that mouse and rat cells lack the receptor required for DT fragment B binding and subsequent translocation of DT fragment A $(48,222)$. In contrast to DT, ExoS was T3S-T equally well taken up by cells of rodent origin, even though the end result was a restrictive pattern of substrate ADPribosylation (197). With regard to T3S-T-ExoS cytotoxicity, the limiting factor does not appear to be bacterial binding or T3S-T of the effector protein, but instead related to how the toxin is targeted by the mammalian cell.

The importance of the finding that T3S-T-ExoS targets proteins differently in rodent and human cells is the identification of a model system to study factors that determine intracellular T3S-effector targeting and may ultimately provide clues to understanding 
eukaryotic cellular trafficking mechanisms. The rodent cell lines (NIH-3T3 and Swiss3T3) used in our studies were found to be less sensitive than the human cell lines (HT29 and $\mathrm{LNCaP}$ ) to the effects of ExoS. Subsequent studies examining the effects of T3S-T-ExoS on morphological alterations found other rodent cell lines (TCMK-1, CHO$\mathrm{K} 1$ ) to be just as sensitive as HT-29 or LNCaP human cell lines to T3S-T-ExoS-induced cell rounding, and simian and human cell lines (Cos-1, Vero, WI-38) to show similar rounding patterns as $\mathrm{NIH}-3 \mathrm{~T} 3$ and Swiss-3T3 cells (personal observation). Despite the differences in sensitivity of individual cell lines to the morphological effects of T3S-TExoS, the patterns of ExoS ADP-ribosylation remained consistent within rodent (restrictive) and human and simian (extensive) cell lines. These qualitative comparisons highlight the notion that while cellular reactions to T3S-T-ExoS vary, ExoS substrate targeting remains constant and dependent on mammalian origin.

In examining the effects of T3S-T-ExoS on different cell lines, HL-60 myeloid cells were found to be resistant to the effects of ExoS on cell function, which corresponded with a lack of ADP-ribosylation of eukaryotic proteins. Differentiation of HL-60 cells with a phorbol ester, TPA, induced HL-60 cell sensitivity to effects of ExoS on cell morphology and ExoS ADP-ribosylation of substrates. Studies examining mechanisms of resistance, including bacterial adherence, induction of $\mathrm{Pa}$ T3S and $\mathrm{Pa}$ T3S-T, revealed that the resistance and TPA-induced sensitivity to ExoS related to Pa T3S-T of ExoS (198). More bacteria were able to adhere to HL-60 cells than to TPA-HL-60 cells, and $\mathrm{HL}-60$ cells were able to induce the production of $\mathrm{PopD}$, a protein required for T3ST. The inability to detect intracellular ExoS-HA in HL-60 cells, which nevertheless bound $\mathrm{Pa}$ and induced PopD, indicated that an HL-60 cell property interfered with T3S-T of 
ExoS. It remains unknown if a functional $\mathrm{Pa}$ T3S apparatus is formed when $\mathrm{Pa}$ is in contact with HL-60 cells.

Previous discussion of HL-60 cell resistance focused on cell surface features that might be altered with TPA-differentiation (Chapter 3). Other studies examining pharmaceutical induction of $\mathrm{Pa}$ T3S sensitivity or resistance showed that treatment of HL-60 cells with colchicine A, which disrupts microtubules, prior to co-culture with ExoS producing bacteria, allowed ADP-ribosylation of Rab5 and RalA (E. Rucks, unpublished data). Treatment of cells with colchicine A did not affect normal cellular distribution of either Rab5 or RalA, as assessed by cell fractionation studies. In addition, treatment of T24 cells with colchicine A also resulted in increased T3S-T of ExoS, compared to untreated cells. Combined, these data suggest that a possible mechanism affecting T3S-T-resistance or sensitivity relates to the cytoskeletal structure of eukaryotic cells interacting with $\mathrm{Pa}$. Polarized epithelial cells, like HL-60 cells, are resistant to Pa T3S-T $(34,58,115,154)$, and both cell types can be induced to become T3S-T-sensitive, through non-polarization or TPA-differentiation, respectively. Polarized epithelial monolayers exhibit an asymmetrical microtubule arrangement with the minus ends of microtubules being anchored at apical surface and plus ends being at the basal surface (9). In contrast, undifferentiated HL-60 cells exhibit a concentrically symmetrical microtubule organization, with microtubules radiating from a central location (136). Upon conversion to T3S-T-sensitivity, non-polarized epithelial monolayers exhibit properties of both symmetrical and asymmetrical microtubule organization, with microtubules radiating from the centrosome and Golgi complex $(9,160)$. Upon TPA differentiation and induction of T3S-T-sensitivity in HL-60 cells, the microtubule network stretches into 
pseudopodia from the centriolar region, in association with a loss of symmetrical arrangement (112). While no obvious microtubule commonality is recognized in T3S-Tresistant cells, alterations in microtubule symmetry may be a feature common to the induction of T3S-T-sensitivity by increasing the efficiency of the T3S-T process.

Recent studies have drawn attention to possible modulatory influences the eukaryotic cell can have on ExoS function. Notably, T3S-T-ExoS ADPRT activity was found to be enhanced in the membrane compartment of epithelial cells $(64,188)$. The enhancement of ExoS ADPRT activity within the cell was further evident in the detection of Ras and RalA modification upon T3S-T of an ExoS ADPRT E381A mutant, which was unable to modify either protein in vitro (64) (Chapter 4). Correlating with these findings was the identification of ExoS having a membrane localization domain (MLD) in ExoS (176). T3S-T of ExoS $\triangle M L D$ lacking this domain results in cytosolic ExoS localization and decreased ADP-ribosylation of euakaryotic substrates (176). These studies indicate the importance of ExoS membrane localization for ADPRT activity, which supports the hypothesis that ExoS membrane localization optimizes interaction of ExoS with eukaryotic cofactor required for ExoS ADPRT activity.

Studies examining the cellular effects of an ExoS GAP R146A mutant also provided evidence that ExoS GAP activity modulates ADPRT function within the eukaryotic cell (64). The complex interactions of the domains is illustrated with Rac1, a LMWG-protein, which has been identified as a target of both ExoS GAP and ADPRT activities $(65,77$, 193). Rac1 regulates multiple cellular processes, including phagocytosis and cell motility, partly by controlling cytoskeleton dynamics. It is a primarily cytosolic protein, but upon activation, GTP-bound Rac1 localizes to the membrane where it interacts with 
downstream effector PAK (229). After T3S-T of ExoS into human epithelial cells, ExoS was found to ADP-ribosylate Rac1, leading to Rac1 activation and membrane association, as indicated by ADP-ribosylated Rac1 interaction with PAK and cellular fractionation analysis, respectively $(65,193)$. In contrast, in macrophages, Rac1 was found to be a target of T3S-T-ExoS GAP, rather than ADPRT, activity, which resulted in inactivation of Rac1 and prevention of phagocytosis (192). Recent studies found that T3S-T-ExoS targeted Rac1 by either GAP or ADPRT activities, but not both, and that these differences in ExoS targeting correlated with subtle differences in Rac1 subcellular localization within human epithelial and murine macrophage cells (193). Combined, these data indicate that the GAP and ADPRT domains of ExoS are modulated within the context of the host cell.

A role of R146A in the GAP domain in the effectiveness of T3S-T of ExoS has also been identified. Mutation of R146A resulted in increased levels of T3S-T-ExoS in eukaryotic membrane and cytosolic fractions. The more efficient translocation of R146A ExoS did not lead to alteration of the substrate modification pattern of T3S-T-ExoS. These data indicate that R146A may have a regulatory role at the level of T3S-T of ExoS and/or subsequent ADPRT activity. Previous studies indicated that R146 was the preferential site of ExoS auto-ADP-ribosylation, but loss of this site does not completely abrogate auto-ADP-ribosylation activities in vitro (189). In our studies we found that the R146A mutant was still efficiently auto-ADP-ribosylated within the eukaryotic cell. It has been reported in eukaryotic ADPRT proteins, auto-ADP-ribosylation serves as a means of regulating enzymatic function (244). Therefore, with mutation of R146, T3S-T-ExoS 
may be auto-ADP-ribosylating at an alternative site that results in disregulation of a feedback mechanism controling the amounts of ExoS that is T3S-T or ADPRT active.

If R146 controls the amount of ExoS that is bacterially translocated, its mutation could have an effect on the translocation of other $\mathrm{Pa}$ T3S effector proteins. The R146A mutation does not result in greater bacterial secretion of mutant ExoS compared to wild type ExoS in eukaryotic cell-free bacterial cultures, suggesting that a host cell process influences altered amounts of T3S-T-ExoS-R146A. This abberation can be studied by monitoring the T3S-T of ExoT or ExoY in strains expressing ExoS-R146A-HA. If ExoSR146 serves as a general regulator of $\mathrm{Pa}$ T3S-T, an increase in the translocation of ExoT or ExoY would be expected in strains expressing ExoS-R146A, as compared to wild type ExoS. Interestingly, ExoT also harbors a GAP domain $(114,122)$, which could function similarly to that of ExoS. If ExoS GAP activity, in combination with a host cell process, regulated intracellular amounts of T3S-T-ExoS, then translocation of wild type ExoT or transfection of the ExoS GAP domain would be expected to result in normal intracellular levels of T3S-T-ExoS R146A GAP mutant relative to intracellular levels of wild type ExoS. In summary, current studies suggest that specific ExoS residues, in combination with host cell processes, influence the translocation and intracellular targeting of ExoS activity.

Current data indicate that T3S-T-ExoS interacts with different proteins, in association with its different ADPRT substrate modification patterns in human and rodent cell lines. Using a Co-IP approach to identify ExoS-associated proteins within eukaryotic cells, ExoS was found to associate with 14-3-3 proteins in both rodent and human cell lines. This finding is consistent with the known interaction of Exos with its 
14-3-3 cofactor in vitro and confirmed the utility of this Co-IP approach to identify ExoS interactive proteins. In addition, ExoS was found to interact with 40 and $45 \mathrm{kDa}$ proteins in human cells and 70 and $85 \mathrm{kDa}$ proteins in rodent cell lines. While the identity of these proteins has not been confirmed, it is expected that their identification will provide insight into the differential subcellular localization of T3S-T-ExoS within rodent and human cells.

The identification of these proteins could also elucidate intracellular trafficking mechanisms of ExoS. In human cell lines, ExoS has been found to ADP-ribosylate Rab proteins, which are located in different subcellular compartments, in a distinct order: first Rab5, then Rabs 8 and 11, and lastly Rab7 (65). Rab5 resides in clathrin coated vesicles and early endosomes; Rab8 resides within the trans-Golgi region and on secretory granules; Rab11 is found within the perinuclear recycling endosomes and recycling endosomes from the Golgi apparatus; and Rab7 is found on late endosomes (267). The timing of ExoS ADP-ribosylation of these Rab proteins supports the notion that ExoS has sequential access to these subcellular locations. Interestingly, Rab4, which was not found to be a target of T3S-T-ExoS ADPRT activity, has been found associated with early sorting endosomes, and is also present on vesicles with Rab5 (early sorting endosomes) and Rab11 (perinuclear recycling endosomes) $(217,267)$. Previous studies indicated that Rab4 is central to early endosomal pathways in the ability to facilitate the trafficking of Rab5 associated vesicles to Rab11 (217). It was also reported that ExoS ADP-ribosylation of Rab5 interfered with protein function, implicating a functional role of ExoS ADP-ribosylation in the targeting of Rab proteins (10). These observations, in combination with lack of ExoS ADP-ribosylation of Rab4, support the 
notion that within human cells ExoS selectively targets Rab proteins in a manner that facilitates intracellular effector transport.

It is currently unknown why ExoS ADPRT activity targeted Rab proteins in human and simian cell lines but not in rodent cell lines. The identification of ExoS-interacting proteins may be the first step to understanding a mechanism of these differences. ExoS requires interaction with the cofactor 14-3-3. These studies have confirmed the interaction of ExoS with 14-3-3 in both rodent and human cell lines, the specific isoforms have not yet been identified. The significance of this would be to determine if ExoS interacts with the same or different 14-3-3 isoforms in rodent and human cell lines. As mentioned previously, only two out of seven 14-3-3 isoforms have identical amino acid sequences in comparison of rodent and human. Any difference in ExoS-143-3 interactions in rodent and human cells might result in different protein interactions and intracellular localization of ExoS. Therefore, understanding these differences would determine a mechanism for different intracellular trafficking of ExoS ADPRT in rodent and human cell lines.

As mentioned previously, T3S-T-ExoS caused eukaryotic cell rounding within 3 to 4 hours of co-culture (170). Initial cell rounding is thought to be caused by ExoS GAP activity disrupting actin filaments (178), while permanent cell rounding has been linked to ExoS ADPRT activity (64). When considering T3S-T-ExoS intracellular trafficking patterns, they must be considered in the context of a modified actin cytoskeleton. Treatment of eukaryotic cells with colchicine A, which disrupts microtubules, enhanced T3S-T of ExoS, similar to the effect of the R146A mutation on T3S-T of ExoS. Unexpectedly, in human epithelial cells that have been treated with colchicine A and co- 
cultured with ExoS producing bacteria, ADP-ribosylation of RalA, Rab5 and Rab7 was observed, indicating that ExoS could still gain access to these subcellular compartments. Recent advances in understanding vesicular trafficking have shown that Rab proteins, through interaction with kinesin-like motor proteins, direct the motility of vesicles and organelles by a microtubule-dependent mechanism $(47,167,168,209)$. Critical to linking T3S-T-ExoS intracellular trafficking to the microtubule cytoskeleton is further characterization of the effects of colchicine A on ExoS substrate targeting and pharmacological abrogation of vesicular trafficking mechanisms through the use of such agents as brefeldin $\mathrm{A}$. It is my current hypothesis that ExoS specifically alters the actin cytoskeleton to allow for intracellular trafficking via a modified microtubule cytoskeleton, and that multiple Rab proteins are ADP-ribosylated in human cells leaving only a subset of Rab vesicles navigable. The subtle role of the host cell in this process is evident in the modulation of ExoS activity via interaction with 14-3-3 and subsequent protein interactions, which results in the restriction (rodent cell lines) or extension (simian and human cell lines) of ExoS intracellular trafficking (Figure 29). 


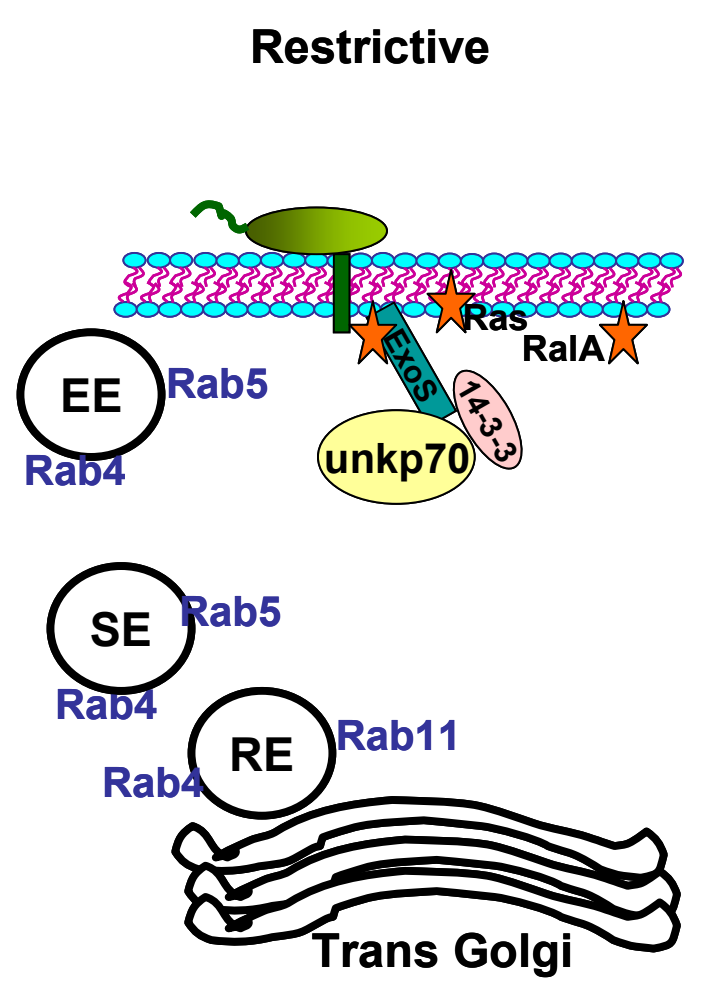

\section{Extensive}

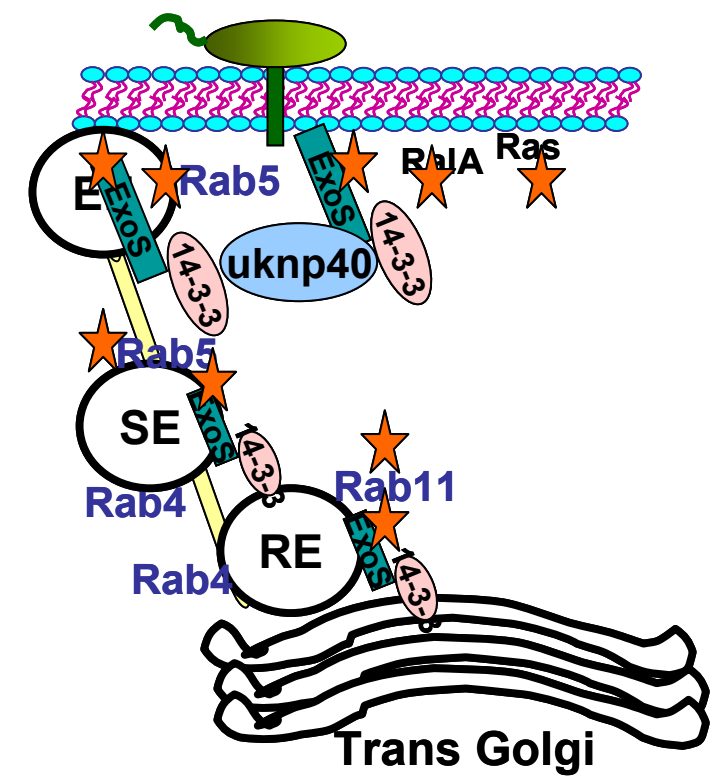

Figure 29. Model of differential targeting of T3S-T-ExoS ADPRT activity

The restrictive pattern characteristic of rodent cells, ExoS is sequestered from vesicles by interaction with a $70 \mathrm{kDa}$ (unkp70) or $85 \mathrm{kDa}$ (not depicted) protein. The extensive pattern, seen in simian or human cells, ExoS interaction with a $40 \mathrm{kDa}$ (unkp40) or 45 $\mathrm{kDa}$ (not depicted) protein aids in targeting ExoS to vesicles, which are then trafficked on microtubule filaments (light yellow cylinders) from the plasma membrane to other intracellular organelles, such as the trans Golgi region. ExoS ADP-ribosylation of itself and eukaryotic proteins is indicated in orange stars. EE, early endosome; SE sorting endosome; RE, recycling endosome.

An recent review written by Marino Zerial and Heidi McBride summarized the function of Rab proteins, which provided the foundation required to relate ExoS ADP-ribosylation patterns to eukaryotic intracellular trafficking mechanisms (267). 
In conclusion, studies described in this dissertation have recognized that the host cell can influence the $\mathrm{Pa}$ T3S-T of ExoS by: 1) determination of ExoS ADPRT-substrate restrictive or extensive modification pattern, 2) allowing of membrane translocation, and 3) targeting intracellular ExoS GAP or ADPRT activity potentially through protein interactions or intracellular trafficking mechanisms. 


\section{$\underline{\text { References }}$}

1. Aitken, A. 1996. 14-3-3 and its possible role in co-ordinating multiple signaling pathways. Trends Cell Biol. 6:341-347.

2. Albus, A. M., E. C. Pesci, L. J. Runyen-Janecky, S. E. H. West, and B. H. Iglewski. 1997. Vfr controls quorum sensing in Pseudomonas aeruginosa. J. Bacteriol. 179:3928-3935.

3. Allewelt, M., F. T. Coleman, M. Grout, G. P. Priebe, and G. B. Pier. 2000. Acquisition of expression of the Pseudomonas aeruginosa ExoU cytotoxin leads to increased bacterial virulence in a murine model of acute pneumonia and systemic spread. Infect. Immun. 68:3998-4004.

4. Allured, V. S., R. J. Collier, S. F. Carroll, and D. B. McKay. 1986. Structure of exotoxin A of Pseudomonas aeruginosa at 3.0-Angstrom resolution. Proc. Natl. Acad. Sci. USA 83:1320-1324.

5. Anderson, D. M., D. E. Fouts, A. Collmer, and O. Schneewind. 1999. Reciprocal secretion of proteins by the bacterial type III machines of plant and animal pathogens suggests universal recognition of mRNA targeting signals. Proc. Natl. Acad. Sci. USA 96:12839-12843.

6. Anderson, D. M., and O. Schneewind. 1999. Yersinia enterocolitica type III secretion: an mRNA signal that couples translation and secretion of YopQ. Mol. Microbiol. 31:1139-1148.

7. Apodaca, G., M. Bomsel, R. a. Lindstedt, J. Engel, D. Frank, K. E. Mostov, and J. Wiener-Kronish. 1995. Characterization of Pseudomonas aeruginosa-induced MDCK cell injury: glycosylation-defective host cells are resistant to bacterial killing. Infect. Immun. 63:1541-1551.

8. Azghani, A. O., J. C. Connelly, B. T. Peterson, L. D. Gray, M. L. Collins, and A. R. Johnson. 1990. Effects of Pseudomonas aeruginosa elastase on alveolar epithelial permeability in guinea pigs. Infect. Immun. 58:433-438.

9. Bacallao, R., C. Antony, C. Dotti, E. Karsenti, E. H. K. Stelzer, and K. Simons. 1989. The subcellular organization of Madin-Darby Canine Kidney cells during the formation of a polarized epithelium. J. Cell. Biol. 109(6):2817-2832. 
10. Barbieri, A. M., Q. Sha, P. Bette-Bobillo, P. D. Stahl, and M. Vidal. 2001. ADPribosylation of rab5 by ExoS of Pseudomonas aeruginosa affects endocytosis. Infect. Immun. 69:5329-5334.

11. Beatrice, J., P. Maud, A. Stephane, C. Francois, G. Frederic, G. Benoit, and M.O. Husson. 2005. Relative expression of Pseudomonas aeruginosa virulence genes analyzed by a real time RT-PCR method during lung infection in rats. FEMS Microbiol. Lett. 243:271-278.

12. Beckmann, C., M. Brittnacher, R. Ernst, N. Mayer-Hamblett, S. I. Miller, and J. L. Burns. 2005. Use of phage display to identify potential Pseudomonas aeruginosa gene products relevant to early cystic fibrosis airway infections. Infect. Immun. 73:444-452.

13. Bergmans, H. E., I. M. vanDie, and W. P. Hoekstra. 1981. Transformation in Escherichia coli:stages in the process. J. Bacteriol. 146:564-570.

14. Bestilny, L. J., and K. T. Riabowol. 2000. A role for serine proteases in mediating phorbol ester-induced differentiation of HL-60 cells. Exper. Cell. Res. 256:264-271.

15. Bette-Bobillo, P., P. Giro, J. Sainte-Marie, and M. Vidal. 1998. Exoenzyme S from $P$. aeruginosa ADP Ribosylates rab4 and inhibits transferrin recycling in SLOpermeabilized reticulocytes. Biochem. Biophys. Res. Comm. 244:336-341.

16. Bleves, S., C. Soscia, P. Nogueira-Orlandi, A. Lazdunski, and A. Filloux. 2005. Quorum sensing negatively controls type III secretion regulon expression in Pseudomonas aeruginosa PAO1. J. Bacteriol. 187:3898-3902.

17. Blocker, A., P. Gounon, E. Larquet, K. Niebuhr, V. Cabiaux, C. Parsot, and P. Sansonetti. 1999. The tripartite type III secretion of Shigella flexneri inserts IpaB and IpaC into host membranes. J. Cell. Biol. 147:683-693.

18. Blocker, A., N. Jouihri, E. Larquet, P. Gounon, F. Ebel, C. Parsot, P. Sansonetti, and A. Allaoui. 2001. Structure and composition of Shigella flexneri 'needle complex', a part of its type III secretion. Mol. Microbiol. 39:652-663.

19. Blocker, A., K. Komoriya, and S.-I. Aizawa. 2003. Type III secretion systems and bacterial flagella: insights into their function from structural similarities. Proc. Natl. Acad. Sci. USA 100:3027-3030. 
20. Boyd, A. P., I. Lambermont, and G. R. Cornelis. 2000. Competition between the Yops of Yersinia enterocolitica for delivery into eukaryotic cells: role of the SycE chaperone binding domain of YopE. J. Bacteriol. 182:4811-4821.

21. Broms, J. E., A.-L. Forslund, A. Forsberg, and M. S. Francis. 2003. PcrH of Pseudomonas aeruginosa is essential for secretion and assembly of the type III translocon. J. Infect. Dis. 188:1909-1921.

22. Burns, D. L. 1999. Biochemistry of type IV secretion. Curr. Opin. Microbiol. 2:2529.

23. Burns, D. L., J. T. Barbieri, B. H. Iglewski, and R. Rappuoli (ed.). 2003. Bacterial protein toxins. American Society for Microbiology, Washington, D.C.

24. Chuang, S.-E., A.-L. Chen, and C.-C. Chao. 1995. Growth of E. coli at low temperature dramatically increases the transformation frequency by electroporation. Nucleic Acid Res. 23:1641.

25. Coburn, J. 1992. Pseudomonas aeruginosa exoenzyme S. Curr. Top. Microbiol. Immunol. 175:133-43.

26. Coburn, J., S. T. Dillon, B. H. Iglewski, and D. M. Gill. 1989a. Exoenzyme S of Pseudomonas aeruginosa ADP-ribosylates the intermediate filament protein vimentin. Infect. Immun. 57:996-998.

27. Coburn, J., and D. W. Frank. 1999. Macrophages and epithelial cells respond differently to the Pseudomonas aeruginosa type III secretion system. Infect. Immun. 67:3151-3154.

28. Coburn, J., and D. M. Gill. 1991. ADP-ribosylation of p21ras and related proteins by Pseudomonas aeruginosa Exoenzyme S. Infect. Immun. 59:4259-4262.

29. Coburn, J., A. V. Kane, L. Feig, and D. M. Gill. 1991. Pseudomonas aeruginosa exoenzyme $S$ requires a eukaryotic protein for ADP-ribosyltransferase activity. J. Biol. Chem. 266:6438-6446.

30. Coburn, J., R. T. Wyatt, B. H. Iglewski, and D. M. Gill. 1989b. Several GTPbinding proteins, including p21 $\mathrm{C}-\mathrm{H}-\mathrm{ras}$, are preferred substrates of Pseudomonas aeruginosa exoenzyme S. J. Biol. Chem. 264:9004-9008. 
31. Collier, R. J., and J. A. M. Pappenheimer. 1964. Studies on the mode of action of diphtheria toxin II. Effect of toxin on amino acid incorporation in cell-free systems. J. Exp. Med. 120:1019-39.

32. Collins, S. J., R. C. Gallo, and R. E. Gallagher. 1977. Continuous growth and differentiation of human myeloid leukaemic cells in suspension culture. Nature 270:347-349.

33. Collins, S. J., F. W. Ruscetti, R. E. Gallagher, and R. C. Gallo. 1978. Terminal differentiation of human promyelocytic leukemia cells induced by dimethyl sulfoxide and other polar compounds. Proc. Natl. Acad. Sci. USA 75:2458-2462.

34. Comolli, J. C., L. L. Waite, K. E. Mostov, and J. E. Engel. 1999. Pili binding to asialo-GM1 on epithelial cells can mediate cytotoxicity or bacterial internalization by Pseudomonas aeruginosa. Infect. Immun. 67:3207-3214.

35. Corda, D., and M. DiGirolamo. 2003. Functional aspects of protein mono-ADPribosylation. EMBO J. 22:1953-8.

36. Cornelis, G. R. 2000a. Molecular and cell biology aspects of plague. Proc. Natl. Acad. Sci. USA 97:8778-8783.

37. Cornelis, G. R. 2000b. Type III secretion: a bacterial device for close combat with cells of their eukaryotic host. Philos. Trans. R. Soc. Lond. B. Biol. Sci. 355:681-693.

38. Cowell, B., D. Chen, D. Frank, A. Vallis, and S. Fleiszig. 2000. ExoT of cytotoxic Pseudomonas aeruginosa prevents uptake by corneal epithelial cells. Infect. Immun. 68:403-406.

39. Cuervo, A. M., and J. F. Dice. 2000. Regulation of lamp2a levels in the lysosomal membrane. Traffic 1:570-583.

40. Dasgupta, N., G. L. Lykken, M. C. Wolfgang, and T. L. Yahr. 2004. A novel antianti-activator mechanism regulates expression of the Pseudomonas aeruginosa type III secretion system. Mol. Microbiol. 53:297-308.

41. deBentzmann, S., P. Roger, F. Dupuit, O. Bajolet-Laudinat, C. Fuchey, M. C. Plotkowski, and E. Puchelle. 1996. Asialo GM1 is a receptor for Pseudomonas aeruginosa adherence to regenerating respiratory epithelial cells. Infect. Immun. 64(5):1582-1588. 
42. deBruin, E. C., d. Meersma, J. deWilde, I. denOtter, E. M. Schipper, J. P. Medema, and L. T. C. Peltenburg. 2003. A serine protease is involved in the initiation of DNA damage-induced apoptosis. Cell Death Diff. 10:1204-1212.

43. Dennis, J. J., and P. A. Sokol. 1995. Electrotransformation of Pseudomonas. Methods Mol. Biol. 47:125-133.

44. DiNovo, A. A., K. L. Schey, W. Vachon, E. M. McGuffie, J. C. Olson, and T. S. Vincent. in review. ADP-ribosylation of cyclophilin A by Pseudomonas aeruginosa exoenzyme S.

45. Donnenberg, M. S. 1999. Salmonella strikes a balance. Nature 401:218-219.

46. Dougherty, M. K., and D. K. Morrison. 2004. Unlocking the code of 14-3-3. J. Cell Sci. 117:1875-1884.

47. Echard, A., F. Jollivet, O. Martinez, J. J. Lacapere, A. Rousselet, I. JanoueixLerosey, and B. Goud. 1998. Interaction of a golgi-associated kinesin-like protein with Rab6. Science 279:580-585.

48. Eidels, L., R. L. Proia, and D. A. Hart. 1983. Membrane receptors for bacterial toxins. Microbiol. Rev. 47:596-620.

49. Elting, L. S., E. B. Rubenstein, K. V. I. Rolston, and G. P. Bodey. 1997. Outcomes of bacteremia in patients with cancer and neutropenia: observations from two decades of epidemiological and clinical trials. Clin. Infect. Dis. 25:247-259.

50. Feig, L. A., T. Urano, and S. Cantor. 1996. Evidence for a Ras/Ral signaling cascade. Trends Biochem. Sci. 21:438-441.

51. Feldman, M. F., S. Muller, E. Wuest, and G. R. Cornelis. 2002. SycE allows secretion of YopE-DHFR hybrids by the Yersinisa enterocolitica type III Ysc system. Mol. Microbiol. 46:1183-1197.

52. Feltman, H., G. Schulert, S. Khan, M. Jain, L. Peterson, and A. R. Hauser. 2001. Prevalence of type III secretion genes in clinical and environmental isolates of Pseudomonas aeruginosa. Microbiology 147:2659-2669.

53. Fendrick, J. L., and W. J. Iglewski. 1989. Endogenous ADP-ribosylation of elongation factor 2 in polyoma virus-transformed baby hamster kidney cells. Proc. Natl. Acad. Sci. USA 86:554-557. 
54. Ferguson, M. W., J. A. Maxwell, T. S. Vincent, J. d. Silva, and J. C. Olson. 2001. Comparison of the exoS gene and protein expression in soil and clinical isolates of Pseudomonas aeruginosa. Infect. Immun. 69:2198-2210.

55. Finck-Barbancon, V., J. Goranson, L. Zhu, T. Sawa, J. P. Weiner-Kronish, S. M. Fleiszig, C. Wu, L. Mende-Mueller, and D. W. Frank. 1997. ExoU expression by Pseudomonas aeruginosa correlates with acute cytotoxicity and epithelial injury. Mol. Microbiol. 25:547-557.

56. Finck-Barbancon, V., T. L. Yahr, and D. W. Frank. 1998. Identification and characterization of $\mathrm{SpcU}$, a chaperone required for efficient secretion of the ExoU cytotoxin. J. Bacteriol. 180:6224-6231.

57. FitzGerald, D., R. E. Morris, and C. B. Saelinger. 1980. Receptor-mediated internalization of Pseudomonas toxin by murine fibroblasts. Cell 21:867-873.

58. Fleiszig, S., D. Evans, N. Do, V. Vallas, S. Shin, and K. Mostov. 1997b. Epithelial cell polarity affects susceptibility to Pseudomonas aeruginosa invasion and cytotoxicity. Infect. Immun. 65:2861-2867.

59. Fleiszig, S. M. J., V. Vallas, C. H. Jun, L. Mok, D. F. Balkovetz, M. G. Roth, and K. E. Mostov. 1998. Susceptibility of epithelial cells to Pseudomonas aeruginosa invasion and cytotoxicity is upregulated by hepatocyte growth factor. Infect. Immun. 66:3443-3446.

60. Frank, D. W. 1997. The exoenzyme S regulon of Pseudomonas aeruginosa. Mol. Microbiol. 26:621-629.

61. Frank, D. W., and B. H. Iglewski. 1991. Cloning and sequence analysis of a transregulatory locus required for exoenzyme $\mathrm{S}$ synthesis in Pseudomonas aeruginosa. J. Bacteriol. 173:6460-6468.

62. Frank, D. W., G. Nair, and H. P. Schweizer. 1994. Construction and characterization of chromosomal insertional mutations of the Pseudomonas aeruginosa exoenzyme S trans-regulatory locus. Infect. Immun. 62:554-563.

63. Fraylick, J. E., J. T. Barbieri, M. J. Riesse, T. S. Vincent, and J. C. Olson. 2002b. ADP-ribosylation and functional effects of Pseudomonas ExoS on cellular Ral. Biochemistry 41:9680-9687. 
64. Fraylick, J. E., J. R. LaRocque, T. S. Vincent, and J. C. Olson. 2001. The independent and coordinate effects of the ADP-ribosyltransferase and GTPaseactivating activities of exoenzyme S on HT-29 epithelial cell function. Infect. Immun. 69:5318-5328.

65. Fraylick, J. E., E. A. Rucks, D. M. Greene, T. S. Vincent, and J. C. Olson. 2002a. Eukaryotic cell determination of ExoS ADP-ribosyltransferase substrate specificity. Biochem. Biophys. Res. Commun. 291:91-100.

66. Frithz-Lindsten, E., Y. Du, R. Rosqvist, and A. Forsberg. 1997. Intracellular targeting of exoenzyme S of Pseudomonas aeruginosa via type III-dependent translocation induces phagocytosis resistance, cytotoxicity and disruption of actin microfilaments. Mol. Microbiol. 25:1125-1139.

67. Frithz-Lindsten, E., A. Holmstrom, L. Jacobsson, M. Soltani, J. Olsson, R. Rosqvist, and A. Forsberg. 1998. Functional conservation of the effector protein translocators PopB/YopB and PopD/YopD of Pseudomonas aeruginosa and Yersinia pseudotuberculosis. Mol. Microbiol. 29:1155-1165.

68. Fu, H., J. Coburn, and R. J. Collier. 1993. The eukaryotic host factor that activates exoenzyme $S$ of Pseudomonas aeruginosa is a member of the 14-3-3 protein family. Proc. Natl. Acad. Sci. USA 90:2320-2324.

69. Fu, Y., and J. E. Galan. 1999. A Salmonella protein antagonizes Rac-1 and Cdc42 to mediate host-cell recovery after bacterial invasion. Nature 401:293-297.

70. Galan, J. E., and A. Collmer. 1999. Type III secretion machines: bacterial devices for protein delivery into host cells. Science 284:1323-1328.

71. Ganesan, A. K., D. W. Frank, R. P. Misra, G. Schmidt, and J. T. Barbieri. 1998. Pseudomonas aeruginosa exoenzyme S ADP-ribosylates ras at multiple sites. J. Biol. Chem. 273:7332-7337.

72. Ganesan, A. K., L. Mende-Mueller, J. Selzer, and J. T. Barbieri. 1999. Pseudomonas aeruginosa exoenzyme $\mathrm{S}$, a double ADP-ribosyltransferase, resembles vertebrate mono-ADP-ribosyltransferases. J. Biol. Chem. 274:95039508.

73. Ganesan, A. K., T. S. Vincent, J. C. Olson, and J. T. Barbieri. 1999.

Pseudomonas aeruginosa exoenzymeS disrupts Ras-mediated signal transduction 
by inhibiting guanine nucleotide exchange factor-catalyzed nucleotide exchange. J. Biol. Chem. 274:21823-21829.

74. Garrity-Ryan, L., B. Kazmierczak, R. Kowal, J. Comolli, A. Hauser, and J. N. Engel. 2000. The arginine finger domain of ExoT contributes to actin cytoskeleton disruption and inhibition of internalization of Pseudomonas aeruginosa by epithelial cells and macrophages. Infect. Immun. 68:7100-7113.

75. Gerceker, A. A., T. Zaidi, P. Marks, D. E. Golan, and G. B. Pier. 2000. Impact of heterogeneity within cultured cells on bacterial invasion: analysis of Pseudomonas aeruginosa and Salmonella enterica serovar typhi entry into MDCK cells by using a green fluorescent protein-labeled cystic fibrosis transmembrane conductance regulator receptor. Infect. Immun. 68:861-870.

76. Ghosh, P. 2004. Process of protein transport by the type III secretion system. Microbiol. Mol. Biol. Rev. 68:771-795.

77. Goehring, U.-M., G. Schmidt, K. J. Pederson, K. Aktories, and J. T. Barbieri. 1999. The $\mathrm{N}$-terminal domain of Pseudomonas aeruginosa exoenzyme $\mathrm{S}$ is a GTPase-activating protein for Rho GTPases. J. Biol. Chem. 274:36369-36372.

78. Goodman, A. L., B. Kulasekara, A. Rietsch, D. Boyd, R. S. Smith, and S. Lory. 2004. A signaling network reciprocally regulates genes associated with acute infection and chronic persistence in Pseudomonas aeruginosa. Development. Cell 7:745-754.

79. Goranson, J., A. K. Hovey, and D. W. Frank. 1997. Functional analysis of exsC and exSB in regulation of exoenzyme S production by Pseudomonas aeruginosa. J. Bacteriol. 179:1646-1654.

80. Goure, J., P. Broz, O. Attree, G. R. Cornelis, and I. Attree. 2005. Protective anti$V$ antibodies inhibit Pseudomonas and Yersinia translocon assembly within host membranes. J. Infect. Dis. 192:218-25.

81. Goure, J., A. Pastor, E. Faudry, J. Chabert, A. Dessen, and I. Attree. 2004. The $V$ antigen of Pseudomonas aeruginosa is required for assembly of the functional PopB/PopD translocation pore in host cell membranes. Infect. Immun. 72:47414750. 
82. Grassme, H., V. Jendrossek, A. Riehle, G. v. Kurthy, J. Berger, H. Schwarz, M. Weller, R. Kolesnick, and E. Gulbins. 2003. Host defense against Pseudomonas aeruginosa requires ceramide-rich membrane rafts. Nat. Med. 9:322-330.

83. Green, S. K., M. N. Schroth, J. J. Cho, S. D. Kominos, and V. B. Vitanza-Jack. 1974. Agricultural plants and soil as a reservoir for Pseudomonas aeruginosa. Appl. Microbiol. 28:987-991.

84. Groscop, J. A., and M. M. Brent. 1964. The effects of selected strains of pigmented microorganisms on small free-living amoebae. Can. J. Microbiol. 10:579584.

85. Gupta, S. K., R. S. Berk, S. Masinick, and L. D. Hazlett. 1994. Pili and lipopolysaccharide of Pseudomonas aeruginosa bind to the glycolipid asialo GM1. Infect. Immun. 62:4572-4579.

86. Hardt, W. D., L. M. Chen, K. E. Schuebel, X. R. Bustelo, and J. E. Galan. 1998. S. typhimurium encodes an activator of Rho GTPases that induce membrane ruffling and nuclear responses in host cells. Cell 93:15-26.

87. Hazlett, L. D., S. Masinick, R. Barrett, and K. Rosol. 1993. Evidence of asialo GM1 as a corneal glycolipid receptor for Pseudomonas aeruginosa adhesion. Infect. Immun. 61:5164-5173.

88. Henderson, I. R., F. Navarro-Garcia, M. Desvaux, R. C. Fernandez, and D. Ala'Aldeen. 2004. Type $V$ protein secretion pathway: the autotransporter story. Microbiol. Mol. Biol. Rev. 68:692-744.

89. Henriksson, M., C. Sundin, A. Jansson, A. Forsberg, R. Palmer, and B. Hallberg. 2002b. Exoenzyme $S$ shows selective ADP-ribosylation and GTPaseactivating protein (GAP) activities towards small GTPases in vivo. Biochem.J. 367:617-28.

90. Henriksson, M. L., M. S. Francis, A. Paden, M. Aili, K. Stefansson, R. Palmer, A. Aitken, and B. Hallberg. 2002a. A nonphosphorylated 14-3-3 binding motif on exoenzyme $S$ that is functional in vivo. Eur. J. Biochem. 269:4921-4929.

91. Henriksson, M. L., R. Rosqvist, M. Telepnev, H. Wolf-Watz, and B. Hallberg. 2000b. Ras effector pathway activation by epidermal growth factor is inhibited in vivo by exoenzyme S ADP-ribosylation of Ras. Biochem. J. 347:217-222. 
92. Henriksson, M. L., U. Troller, and B. Hallberg. 2000a. 14-3-3 proteins are required for the inhibition of Ras by exoenzyme S. Biochem. J. 349:697-701.

93. Hoe, N., F. Minion, and J. Goguen. 1992. Temperature sensing in Yersinia pestis: regulation of yopE transcription by IcrF. J. Bacteriol. 174:4275-4286.

94. Hoiczyk, E., and G. Blobel. 2001. Polymerization of a single protein of the pathogen Yersinia enterocolitica into needle punctures eukaryotic cells. Proc. Natl. Acad. Sci. USA 98:4669.

95. Holder, I. A., A. N. Neely, and D. W. Frank. 2001. PcrV immunization enhances survival of burned Pseudomonas aeruginosa-infected mice. Infect. Immun. 69:59085910.

96. Holder, I. A., A. N. Neely, and D. W. Frank. 2001. Type III secretion/intoxication system important in virulence of Pseudomonas aeruginosa infections in burns. Burns 27:129-130.

97. Holmes, D. S., and M. Quigley. 1981. A rapid boiling method for the preparation of bacterial plasmids. Anal. Biochem. 114:193-197.

98. Horoszewicz, J. S., S. S. Leong, T. M. Chu, Z. L. Wajsman, M. Friedman, L. Papsidero, U. Kim, L. S. Chai, S. Kakati, S. K. Arya, and A. A. Sandberg. 1980. The LNCaP cell line- a new model for studies in human prostatic carcinoma, $p$. 115-132. In G. P. Murphy (ed.), Models for Prostate Cancer. Alan R. Liss Inc., New York.

99. Hovey, A. K., and D. W. Frank. 1995. Analyses of the DNA-binding and transcriptional activation properties of ExsA, the transcriptional activator of Pseudomonas aeruginosa exoenzyme S regulon. J. Bacteriol. 177:4427-4436.

100. Huang, H. Y., D. J. Graves, R. M. Robson, and T. W. Huiatt. 1993. ADPribosylation of the intermediate filament protein desmin and inhibition of desmin assembly in vitro by muscle ADP-ribosyltransferase. Biochem. Biophys. Res. Commun. 197:570-577.

101. Huang, H. Y., H. Zhou, T. W. Huiatt, and D. J. Graves. 1996. Target proteins for arginine-specific mono(ADP-ribosyl) transferase in membrane fractions from chick skeletal muscle cells. Exp. Cell Res. 226:147. 
102. Hueck, C. J. 1998. Type III protein secretion systems in bacterial pathogens of animals and plants. Microbiol. Mol. Biol. Rev. 62:379-433.

103. Hutchison, M. L., and J. R. W. Govan. 1999. Pathogenicity of microbes associated with cystic fibrosis. Microbes Infect. 1:1005-1014.

104. Iglewski, B. H. 1988. Pseudomonas toxins, p. 249-265. In M. C. Hardegree and A. T. Tu (ed.), Handbook of toxins, vol. 4. Marcel Dekker, New York.

105. Iglewski, B. H., and D. Kabat. 1975. NAD-dependent inhibition of protein synthesis by Pseudomonas aeruginosa toxin. Proc. Natl. Acad. Sci. USA 72:22842288.

106. Iglewski, B. H., J. Sadoff, M. J. Bjorn, and E. S. Maxwell. 1978.

Pseudomonas aeruginosa exoenzyme S: an adenosine diphosphate ribosyltransferase distinct from toxin A. Proc. Natl. Acad. Sci. USA 75:3211-3225.

107. Imundo, L., J. Barasch, A. Prince, and Q. Al-Awqati. 1995. Cystic fibrosis epithelial cells have a receptor for pathogenic bacteria on their apical surface. Proc. Natl. Acad. Sci. USA 92:3019-3023.

108. Jain, M., D. Ramirez, R. Seshadri, J. F. Cullina, C. A. Powers, G. S. Schulert, M. Bar-Meir, C. L. Sullivan, S. A. McColley, and A. R. Hauser. 2004. Type III secretion phenotypes of Pseudomonas aeruginosa strains change during infection of individuals with cystic fibrosis. J. Clin. Microbiol. 42:5229-5237.

109. Jinno, Y., M. Ogata, V. K. Chaudhary, M. C. Willingham, S. Adhya, D. FitzGerald, and I. Pastan. 1989. Domain II mutants of Pseudomonas exotoxin deficient in translocation. J. Biol. Chem. 264:15953-15959.

110. Journet, L., K. T. Hughes, and G. R. Cornelis. 2005. Type III secretion: a secretory pathway serving both motility and virulence (Review). Mol. Membr. Biol. 22:41-50.

111. Katagiri, K., S. Hattori, S. Nakamura, T. Yamamoto, T. Yoshida, and T. Katagiri. 1994. Activation of ras and formation of GAP complex during TPA-induced monocytic differentiation of HL-60 cells. Blood 84:1780-1789.

112. Katagiri, K., T. Katagiri, K. Kajiyama, T. Yamamoto, and T. Yoshida. 1993. Tyrosine-phosphorylation of tubulin during monocytic differentiation of HL-60 cells. J. Immunol. 150:585. 
113. Katsuragi, N., N. Takizawa, and Y. Murooka. 1987. Entire nucleotide sequence of pullulanse gene of Klebsiella aerogenes W70. J. Bacteriol. 169:23012306.

114. Kazmierczak, B., T. Jou, K. Mostov, and J. Engel. 2001. Rho GTPase activity modulates Pseudomonas aeruginosa internalization by epithelial cells. Cell. Microbiol. 3:85-98.

115. Kazmierczak, B. I., K. Mostov, and J. N. Engel. 2001. Interaction of bacterial pathogens with polarized epithelium. Annu. Rev. Microbiol. 55:407-35.

116. Kimbrough, T. G., and S. I. Miller. 2000. Contribution of Salmonella typhimurium type III secretion components to needle complex formation. Proc. Natl. Acad. Sci. USA 97:11008-11013.

117. Knight, D. A., V. Finck-Barbancon, S. M. Kulich, and J. T. Barbieri. 1995. Functional domains of Pseudomonas aeruginosa exoenzyme S. Infect. Immun. 63:3182-3186.

118. Kominos, S. D., C. E. Copeland, B. Grosiak, and B. Postic. 1972. Introduction of Pseudomonas aeruginosa into hospital via vegetables. Appl. Microbiol. 24:567-570.

119. Koster, M., W. Bitter, H. d. Cock, A. Allaoui, G. R. Cornelis, and J. Tommassen. 1997. The outer membrane component, YscC, of the Yop secretion machinery of Yersinia enterocolitica forms a ring-shaped multimeric complex. Mol. Microbiol. 26:789-797.

120. Kounnas, M. Z., R. E. Morris, M. R. Thompson, D. J. FitzGerald, D. K.

Strickland, and C. B. Saelinger. 1992. The $\alpha_{2}$-macroglobulin receptor/low density lipoprotein receptor-related protein binds and internalizes Pseudomonas exotoxin A. J. Biol. Chem. 267:12420-12423.

121. Kowalski, M. P., and G. B. Pier. 2004. Localization of cystic fibrosis transmembrane conductance regulator to lipid rafts of epithelial cells is required for Pseudomonas aeruginosa-induced cellular activation. J. Immunol. 172:418-425.

122. Krall, R., G. Schmidt, K. Aktories, and J. T. Barbieri. 2000. Pseudomonas aeruginosa ExoT is a Rho GTPase-activating protein. Infect. Immun. 68:6066-6068. 
123. Krivan, H. C., V. Ginsburg, and D. D. Roberts. 1988. Pseudomonas aeruginosa and Pseudomonas cepacia isolated from cystic fibrosis patients bind specifically to gangliotetraosylceramide (asialo GM1) and gangliotriaosylceramide (asiolo GM2). Arch. Biochem. Biophys. 260:493-496.

124. Kubori, T., Y. Matsushima, D. Nakamura, J. Uralil, M. Lara-Tejero, A. Sukhan, J. E. Galan, and S.-I. Aizawa. 1998. Supramolecular structure of the Salmonella typhimurium type III protein secretion system. Science 280:602-605.

125. Kuchma, S. L., J. P. Connolly, and G. A. O'Toole. 2005. A three-component regulatory system regulates biofilm maturation and type III secretion in Pseudomonas aeruginosa. J. Bacteriol 187:1441-1454.

126. Kulich, S. M., D. W. Frank, and J. T. Barbieri. 1995. Expression of recombinant exoenzyme $S$ of Pseudomonas aeruginosa. Infect. Immun. 63:1-8.

127. Kulich, S. M., D. W. Frank, and J. T. Barbieri. 1993. Purification and characterization of exoenzyme $S$ from Pseudomonas aeruginosa388. Infect. Immun. 61:307-313.

128. Kulich, S. M., T. L. Yahr, L. M. Mende-Mueller, J. T. Barbieri, and D. W. Frank. 1994. Cloning the structural gene for the 49-kDa form of exoenzymeS (exoS) from Pseudomonas aeruginosa strain 388. J. Biol. Chem. 269:10431-10437.

129. Laemmli, U. K. 1970. Cleavage of structural proteins during the assembly of the head of bacteriophage T4. Nature (London) 227:680-685.

130. LambertDERouvroit, C., C. Sluiters, and G. R. Cornelis. 1992. Role of the transcriptional activator, VirF, and temperature in the expression of the pYV plasmid genes of Yersinia enterocolitica. Mol. Microbiol. 6:395-409.

131. Laouar, A., F. R. Collart, C. B. H. Chubb, B. Xie, and E. Huberman. 1999. Interaction between $\alpha 5 \beta 1$ integrin and secreted fibronectin is involved in macrophage differentiation of human HL-60 myeloid leukemia cells. J. Immunol. 162:407-414.

132. Lecuit, M., S. Dramsi, C. Gottardi, M. Fedor-Chaiken, B. Gumbiner, and P. Cossart. 1999. A single amino acid in E-cadherin responsible for host specificity towards the human pathogen Listeria monocytogenes. EMBO J. 18:3956-3963. 
133. Lee, A., D. Chow, B. Haus, W. Tseng, D. Evans, S. Fleiszig, G. Chandy, and T. Machen. 1999. Airway epithelial tight junctions and binding cytotoxicity of Pseudomonas aeruginosa. Am. J. Physiol. 277:L204-L217.

134. Leglise, M. C., G. A. Dent, L. H. Ayscue, and D. W. Ross. 1988. Leukemic cell maturation: phenotypic variability and oncogene expression in HL-60 cells: a review. Blood Cells 13:319-337.

135. Leroy-Dudal, J., H. Gagniere, E. Cossard, F. Carreiras, and P. D. Martino. 2004. Role of $\alpha \vee \beta 5$ integrins and vitronectin in Pseudomonas aeruginosa PAK interaction with A549 respiratory cells. Microb. Infect. 6:875-881.

136. Leung, M.-F., J. A. Sokoloski, and A. C. Sartorelli. 1992. Changes in microtubules, microtubule-associated proteins, and intermediate filaments during the differentiation of HL-60 leukemia cells. Cancer Res. 52:949-952.

137. Linderoth, N. A., M. N. Simon, and M. Russel. 1997. The filamentous phage pIV multimer visualized by scanning transmission electron microscopy. Science 278:1635-1638.

138. Liu, P. V. 1974. Extracellular toxins of Pseudomonas aeruginosa. J. Infect. Dis. 130:S94-S99.

139. Liu, P. V. 1964. Factors that influence toxigenicity of Pseudomonas aeruginosa. J. Bacteriol. 88:1421-1427.

140. Liu, P. V. 1966. The roles of various fractions of Pseudomonas aeruginosa in its pathogenesis. J. Infect. Dis. 116:112-116.

141. Liu, S., S. M. Kulich, and J. T. Barbieri. 1996. Identification of glutamic acid 381 as a candidate active site residue of Pseudomonas aeruginosa Exoenzyme S. Biochemistry 35:2754-2758.

142. Lloyd, S. A., M. Norman, R. Rosqvist, and H. Wolf-Watz. 2001. Yersinia YopE is targeted for type III secretion by N-terminal, not mRNA, signals. Mol. Microbiol. 39:520-531.

143. Luzar, M. A., and T. C. Montie. 1985. Avirulence and altered physiological properties of cystic fibrosis strains of Pseudomonas aeruginosa. Infect. Immun. 50:572-576. 
144. Luzar, M. A., M. J. Thomassen, and T. C. Montie. 1985. Flagella and motility alterations in Pseudomonas aeruginosa strains from patients with cystic fibrosis: relationship to patient clinical condition. Infect. Immun. 50:577-582.

145. Lyczak, J. B., C. L. Cannon, and G. B. Pier. 2000. Establishment of Pseudomonas aeruginosa infection: lessons from a versatile opportunist. Microbes Infect. 2:1051-1060.

146. Lyczak, J. B., C. L. Cannon, and G. B. Pier. 2002. Lung infections in cystic fibrosis. Clin. Microbiol. Rev. 15:194-222.

147. Macnab, R. M. 2003. How bacteria assemble flagella. Annu. Rev. Microbiol. 57:77-100.

148. Mahenthiralingham, E., M. E. Campbell, and D. P. Speer. 1994. Nonmotility and phagocytic resistance of Pseudomonas aeruginosa isolates from chronically colonized patients with cystic fibrosis. Infect. Immun. 62:596-605.

149. Manes, S., G. d. Real, and C. Martinez-A. 2003. Pathogens: Raft hijackers. Nat. Rev. Immunol. 3:557-568.

150. Maresso, A. W., M. R. Baldwin, and J. T. Barbieri. 2004. Ezrin/radixin/moesin proteins are high affinity targets for ADP-ribosylation by Pseudomonas aeruginosa ExoS. J. Biol. Chem. 279:38402-38408.

151. Maresso, A. W., M. J. Riese, and J. T. Barbieri. 2003. Molecular heterogeneity of a type III cytotoxin, Pseudomonas aeruginosa exoenzyme S. Biochemistry 42:14249-14257.

152. McCaw, M. L., G. L. Lykken, P. K. Singh, and T. L. Yahr. 2002. ExsD is a negative regulator of the Pseudomonas aeruginosa type III secretion regulon. Mol. Microbiol. 46:1123-1133.

153. McGuffie, E. M., D. W. Frank, T. S. Vincent, and J. C. Olson. 1998. Modification of Ras in eukaryotic cells by Pseudomonas aeruginosa exoenzyme S. Infect. Immun. 66:2607-2613.

154. McGuffie, E. M., J. E. Fraylick, D. J. Hazen-Martin, T. S. Vincent, and J. C. Olson. 1999. Differential sensitivity of human epithelial cells to Pseudomonas aeruginosa exoenzyme S. Infect. Immun. 67:3494-3503. 
155. Michiels, T., J.-C. Vanootenghem, C. L. D. Rouvroit, B. China, A. Gustin, P. Boudry, and G. R. Cornelis. 1991. Analysis of virC, an operon involved in the secretion of Yop proteins by Yersinia enterolitica. J. Bacteriol. 173:4994-5009.

156. Michiels, T., P. Wattiau, R. Brasseur, J.-M. Ruysschaert, and G. Cornelis. 1990. Secretion of Yop proteins by Yersiniae. Infect. Immun. 58:2840-2849.

157. Middlebrook, J. L., and R. B. Dorland. 1977. Response of cultured mammalian cells to the exotoxins of Pseudomonas aeruginosa and Corynebacterium diphtheriae: differential cytotoxicity. Can. J. Microbiol. 23:183-189. 158. Moskalenko, S., D. O. Henry, C. Rosse, G. Mirey, J. H. Camonis, and M. A. White. 2002. The exocyst is a ral effector complex. Nat. Cell Biol. 4:66-72.

159. Mota, L. J., L. Journet, I. Sorg, C. Agrain, and G. R. Cornelis. 2005. Bacterial injectisomes: needle length does matter. Science 307:1278.

160. Musch, A. 2004. Microtubule organization and function in epithelial cells. Traffic $5(1): 1-9$.

161. Neely, A. N., I. A. Holder, J. P. Wiener-Kronish, and T. Sawa. 2005. Passive anti-PcrV treatment protects burned mice against Pseudomonas aeruginosa challenge. Burns 31:153-158.

162. Nicas, T. I., J. Bradley, J. E. Lochner, and B. H. Iglewski. 1985b. The role of exoenzyme $S$ in infections with Pseudomonas aeruginosa. J. Infect. Dis. 152:716721.

163. Nicas, T. I., and B. H. Iglewski. 1985a. Contribution of exoenzymeS to the virulence of Pseudomonas aeruginosa. Antibiot. Chemother. 36:40-48.

164. Nicas, T. I., and B. H. Iglewski. 1985c. The contribution of exoproducts to virulence of Pseudomonas aeruginosa. Can. J. Microbiol. 31:387-392.

165. Nicas, T. I., and B. H. Iglewski. 1984. Isolation and characterization of transposon-induced mutants of Pseudomonas aeruginosa deficient in production of exoenzyme S. Infect. Immun. 45:470-474.

166. Nicas, T. I., and B. H. Iglewski. 1986. Production of elastase and other exoproducts by environmental isolates of Pseudomonas aeruginosa. J. Clin. Microbiol. 23:967-969. 
167. Nielsen, E., F. Severin, J. M. Backer, A. A. Hyman, and M. Zerial. 1999. Rab5 regulates motility of early endosomes on microtubules. Nat. Cell Biol. 1:376382.

168. Nielsen, E., F. Severin, A. A. Hyman, and M. Zerial. 2001. In vitro reconstitution of endosome motility along microtubules. Methods Mol. Biol. 164:133146.

169. Olson, J. C., J. E. Fraylick, E. M. McGuffie, K. M. Dolan, T. L. Yahr, D. W. Frank, and T. S. Vincent. 1999. Interruption of multiple cellular processes in HT-29 epithelial cells by Pseudomonas aeruginosa exoenzyme S. Infect. Immun. 67:28472854.

170. Olson, J. C., E. M. McGuffie, and D. W. Frank. 1997. Effects of differential expression of the 49-kilodalton exoenzyme $\mathrm{S}$ by Pseudomonas aeruginosa on cultured eukaryotic cells. Infect. Immun. 65:248-256.

171. O'Toole, G. A., and R. Kolter. 1998. Flagellar and twitching motility are necessary for Pseudomonas aeruginosa biofilm development. Mol. Microbiol. 30:295-304.

172. Page, A.-L., and C. Parsot. 2002. Chaperones of the type III secretion pathway: jacks of all trades. Mol. Microbiol. 46:1-11.

173. Parkins, M. D., H. Ceri, and D. G. Storey. 2001. Pseudomonas aeruginosa GacA, a factor in multihost virulence, is also essential for biofilm formation. Mol. Microbiol. 40:1215-1226.

174. Pavlovskis, O. R., and B. Wretlind. 1979. Assessment of protease (elastase) as a Pseudomonas aeruginosa virulence factor in experimental mouse burn infection. Infect. Immun. 24:181-187.

175. Pearson, J. P., M. Feldman, B. H. Iglewski, and A. Prince. 2000.

Pseudomonas aeruginosa cell-to-cell signaling is required for virulence in a model of acute pulmonary infection. Infect. Immun. 68(7):4331-4334.

176. Pederson, K. J., R. Krall, M. J. Riese, and J. T. Barbieri. 2002. Intracellular localization modulates targeting of ExoS, a type III cytotoxin, to eukaryotic signaling proteins. Mol. Microbiol. 46:1381-1390. 
177. Pederson, K. J., S. Pal, A. J. Vallis, D. W. Frank, and J. T. Barbieri. 2000. Intracellular localization and processing of Pseudomonas aeruginosa ExoS in eukaryotic cells. Mol. Microbiol. 37:287-299.

178. Pederson, K. J., A. J. Vallis, K. Aktories, D. W. Frank, and J. T. Barbieri. 1999. The amino-terminal domain of Pseudomonas aeruginosa ExoS disrupts actin filaments via small-molecular weight GTP-binding proteins. Mol. Microbiol. 32:393401.

179. Pesci, E. C., J. P. Pearson, P. C. Seed, and B. H. Iglewski. 1997. Regulation of las and $r h l$ quorum sensing in Pseudomonas aeruginosa. J. Bacteriol. 179:31273132.

180. Pier, G. B., M. Gout, and T. S. Zaidi. 1997. Cystic fibrosis transmembrane conductance regulator is an epithelial cell receptor for clearance of Pseudomonas aeruginosa from the lung. Proc. Natl. Acad. Sci. USA 94:12088-12093.

181. Pier, G. B., and R. Ramphal. 2005. Pseudomonas aeruginosa, p. 2587-2616, Mandell, Douglas, and Bennett's principles and practice of infectious diseases, 6 ed. Elsevier/Churchill Livingstone, New York.

182. Plotkowski, M. C., S. d. Bentzmann, S. H. M. Pereira, J.-M. Zahm, O. Bajolet-Laudinat, P. Roger, and E. Puchelle. 1999. Pseudomonas aeruginosa internalization by human epithelial respiratory cells depends on cell differentiation, polarity, and junctional complex integrity. Am. J. Respir. Cell. Mol. Biol. 20:880-890.

183. Pohlman, R. F., H. D. Genetti, and S. C. Winans. 1994. Common ancestry between IncN conjugal transfer genes and macromolecular export systems of plant and animal pathogens. Mol. Microbiol. 14:655-668.

184. Polytarchou, C., and E. Papadimitriou. 2004. Antioxidants inhibit angiogenesis in vivo through down-regulation of nitric oxide synthase expression and activity. Free Radic. Res. 38:501-508.

185. Prince, A. 1992. Adhesins and receptors of Pseudomonas aeruginosa associated with infection of the respiratory tract. Microb. Pathogen. 13:251-260.

186. Radke, J., K. J. Pederson, and J. T. Barbieri. 1999. Pseudomonas aeruginosa exoenzyme $S$ is a biglutamic acid ADP-ribosyltransferase. Infect. Immun. 67:1508-1510. 
187. Rebiere-Huet, J., P. D. Martino, and C. Hulen. 2004. Inhibition of Pseudomonas aeruginosa adhesion to fibronectin by PA-IL and monosaccharides: involvment of a lectin-like process. Can. J. Microbiol. 50:303-312.

188. Riese, M. J., and J. T. Barbieri. 2002. Membrane localization contributes to the in vivo ADP-ribosylation of Ras by Pseudomonas aeruginosa ExoS. Infect. Immun. 70:2230-32.

189. Riese, M. J., U.-M. Goerhring, M. E. Ehrmantraut, J. Moss, J. T. Barbieri, K. Aktories, and G. Schmidt. 2002. Auto-ADP-ribosylation of Pseudomonas aeruginosa ExoS. J. Biol. Chem. 277:12082-12088.

190. Riese, M. J., A. Wittinghofer, and J. T. Barbieri. 2001. ADP ribosylation of arg41 of rap by ExoS inhibits the ability of rap to interact with its guanine nucleotide exchange factor, C3G. Biochemistry 40:3289-3294.

191. Rietsch, A., I. Vallet-Gely, S. L. Dove, and J. J. Mekalanos. 2005. ExsE, a secreted regulator of type III secretion genes in Pseudomonas aeruginosa. Proc. Natl. Acad. Sci. USA 102:8006-8011.

192. Rocha, C. L., J. Coburn, E. A. Rucks, and J. C. Olson. 2003.

Characterization of Pseudomonas aeruginosa exoenzyme $S$ as a bifunctional enzyme in J774A.1 macrophages. Infect. Immun. 71:5296-5305.

193. Rocha, C. L., E. A. Rucks, D. Vincent, and J. C. Olson. 2005. Examination of the coordinate effects of Pseudomonas ExoS on Rac1. Infect. Immun. 73:54585467.

194. Rodman, J. S., and A. Wandinger-Ness. 2000. Rab GTPases coordinate endocytosis. J. Cell Sci. 113:183-192.

195. Roger, P., E. Puchelle, O. Bajolet-Laudinat, J.-M. Tournier, C. Debordeaux, M.-C. Plotowski, J. H. M. Cohen, D. Sheppard, and S. d. Bentzmann. 1999. Fibronectin and $\alpha 5 \beta 1$ integrin mediate binding of Pseudomonas aeruginosa to repairing airway epithelium. Eur. Respir. J. 13(6):1301-1309.

196. Roy-Burman, A., R. H. Savel, S. Racine, B. L. Swanson, N. S. Revadigar, J. Fujimoto, T. Sawa, D. W. Frank, and J. P. Wiener-Kronish. 2001. Type III protein secretion is associated with death in lower respiratory and systemic Pseudomonas aeruginosa infections. J. Infect. Dis. 183:1767-74. 
197. Rucks, E. A., J. E. Fraylick, L. M. Brandt, T. S. Vincent, and J. C. Olson. 2003. Cell line differences in bacterially translocated ExoS ADP-ribosyltransferase substrate specificity. Microbiology 149:319-331.

198. Rucks, E. A., and J. C. Olson. 2005. Characterization of an ExoS type III translocation-resistant cell line. Infect. Immun. 73:638-643.

199. Ruimy, R., E. Genauzeau, C. Barnabe, A. Beaulieu, M. Tibayrenc, and A. Andremont. 2001. Genetic diversity of Pseudomonas aeruginosa strains isolated from ventilated patients with nosocomial pneumonia, cancer patients with bacteremia, and environmental water. Infect. Immun. 69:584-588.

200. Rumbaugh, K. P., J. A. Griswold, and A. N. Hamood. 1999. Pseudomonas aeruginosa strains obtained from patients with tracheal, urinary tract and wound infections: variations in virulence factors and virulence genes. J. Hosp. Infect. 43:211-218.

201. Rumbaugh, K. P., J. A. Griswold, B. H. Iglewski, and A. H. Hamood. 1999. Contribution of quorum sensing to the virulence of Pseudomonas aeruginosa in burn wound infections. Infect. Immun. 67(11):5854-5862.

202. Saiman, L., G. Cacalano, D. Gruenert, and A. Prince. 1992. Comparison of adherence of Pseudomonas aeruginosa to respiratory epithelial cells from cystic fribrosis patients and healthy subjects. Infect. Immun. 60:2808-2814.

203. Saiman, L., K. Ishimoto, S. Lory, and A. Prince. 1990. The effect of piliation and exoproduct expression on the adherence of Pseudomonas aeruginosa to respiratory epithelial monolayers. J. Infect. Dis. 161:541-548.

204. Saiman, L., and A. Prince. 1993. Pseudomonas aeruginosa pili bind to asialoGM1 which is increased on the surface of cystic fibrosis epithelial cells. J.

Clin. Invest. 92:1875-1880.

205. Salmond, G. P. C., and P. J. Reeves. 1993. Membrane traffic wardens and protein secretion in gram-negative bacteria. Trends Biochem. Sci. 18:7-12.

206. Sato, H., D. W. Frank, C. J. Hillard, J. B. Felix, R. R. Pankhaniya, K. Moriyama, V. Finck-Barbancon, A. Buchaklian, M. Lei, R. M. Long, J. WeinerKronish, and T. Sawa. 2003. The mechanism of action of the Pseudomonas aeruginosa-encoded type III cytotoxin, ExoU. EMBO J. 22:2959-2969. 
207. Sato, H., K. Okinaga, and H. Saito. 1988. Role of pili in the pathogenesis of Pseudomonas aeruginosa burn infection. Microbiol. Immunol. 32:131-139.

208. Sawa, T., T. L. Yahr, M. Ohara, K. Kurahashi, M. A. Gropper, J. P. WienerKronish, and D. W. Frank. 1999. Active and passive immunization with Pseudomonas $\mathrm{V}$ antigen protects against type III intoxication and lung injury. Nat. Med. 5:392-398.

209. Schnapp, B. J. 2003. Trafficking of signaling modules by kinesin motors. J. Cell Sci. 116:2125-2135.

210. Schoehn, G., A. M. DiGuilmi, D. Lamaire, I. Attree, W. Weissenhorn, and A. Dessen. 2003. Oligermization of type III secretion proteins PopB and PopD precedes pore formation in Pseudomonas. EMBO J. 22:4957-4967.

211. Schweizer, H. P. 1991. Escherichia-Pseudomonas shuttle vectors derived from pUC18/19. Gene 97:109-112.

212. Shaver, C. M., and A. R. Hauser. 2004. Relative contributions of Pseudomonas aeruginosa ExoU, ExoS, and ExoT to virulence in the lung. Infect. Immun. 72:6969-6977.

213. Simons, K., and E. Ikonen. 1997. Functional rafts in cell membranes. Nature 387:569-572.

214. Singh, P. K., A. L. Schaefer, M. R. Parsek, T. O. Monlinger, M. J. Welsh, and E. P. Greenberg. 2000. Quorum-sensing signals indicate that cystic fibrosis lungs are infected with bacterial biofilms. Nature 407:762-764.

215. Smith, R. S., M. C. Wolfgang, and S. Lory. 2004. An adenylate cyclasecontrolled signaling network regulates Pseudomonas aeruginosa virulence in a mouse model of acute pneumonia. Infect. Immun. 72:1677-1684.

216. Sokol, P. A., C. D. Cox, and B. H. Iglewski. 1982. Pseudomonas aeruginosa mutants altered in their sensitivity to the effect of iron on toxin $A$ or elastase yields. J. Bacteriol. 151:783-787.

217. Sonnichsen, B., S. DeRenzis, E. Nielsen, J. Rietdorf, and M. Zerial. 2000. Distinct membrane domains on endosomes in the recycling pathway visualized by multicolor imaging of Rab4, Rab5, and Rab11. J. Cell Biol. 149:901-914. 
218. Sory, M.-P., A. Boland, I. Lambermont, and G. R. Cornelis. 1995. Identification of the YopE and YopH domains required for secretion and internalization into the cytosol of macrophages, using the cyaA gene fusion approach. Proc. Natl. Acad. Sci. USA 92:1198-12002.

219. Stathopoulos, C., D. R. Hendrixson, D. G. Thanassi, S. J. Hultgren, J. W. S. G. III, and R. C. III. 2000. Secretion of virulence determinants by the general secretory pathway in Gram-negative pathogens: an evolving story. Microbes Infect. 2:1061-1072.

220. Stebbins, C. E., and J. E. Galan. 2001. Maintenance of an unfolded polypeptide by a cognate chaperone in bacterial type III secretion. Nature 414:7781.

221. Stebbins, C. E., and J. E. Galan. 2001. Structural mimicry in bacterial virulence. Nature 412:701-705.

222. Stenmark, H., S. Olsnes, and K. Sandvig. 1988. Requirement of specific receptors for efficient translocation of diphtheria toxin A fragment across the plasma membrane. J. Biol. Chem. 263:13449-13455.

223. Stevens, L. A., J. Moss, M. Vaughan, M. Pizza, and R. Rappuoli. 1999. Effects of site-directed mutagenesis of Escherichia coli heat-labile enterotoxin on ADP-ribosyltransferase activity and interaction with ADP-ribosylation factors. Infect. Immun. 67:259-265.

224. Stieritz, D. D., and I. A. Holder. 1975. Experimental studies of the pathogenesis of infections due to Pseudomonas aeruginosa: description of a burned mouse model. J. Infect. Dis. 131:688-691.

225. Stover, C. K., X. Q. Pham, A. L. Erwin, S. D. Mizoguchi, P. Warrener, M. J. Hickey, F. S. L. Brinkman, W. O. Hugnagle, D. J. Kowalik, M. Lagrou, R. L. Graber, L. Goltry, E. Tolentino, S. Westbrock-Wadman, Y. Yuan, L. L. Brody, S. N. Coulter, K. R. Folger, A. Kas, K. Larbig, R. Lim, K. Smith, D. Spencer, G. K.S. Wong, Z. Wu, I. T. Paulsen, J. Reizer, M. H. Saier, R. E. W. Hancock, S. Lory, and M. V. Olson. 2000. Complete genome sequence of Pseudomonas aeruginosa PA01, an opportunistic pathogen. Nature 406:959-964. 
226. Sugihara, K., S. Asano, K. Tanaka, A. Iwamatsu, K. Okawa, and Y. Ohta. 2002. The exocyst complex binds the small GTPase RalA to mediate filopodia formation. Nat.Cell Biol. 4:73-78.

227. Suh, S.-J., L. J. Runyen-Janecky, T. C. Maleniak, P. Hager, C. H. MacGregor, N. A. Zielinski-Mozny, J. Paul V. Phibbs, and S. E. H. West. 2002. Effect of vfr mutation on global gene expression and catabolite repression control of Pseudomonas aeruginosa. Microbiology 148:1561-1569.

228. Sukhan, A., T. Kubori, J. Wilson, and J. E. Galan. 2001. Genetic analysis of assembly of the Salmonella enterica serovar typhimurium type III secretionassociated needle complex. J. Bacteriol. 183:1159-1167.

229. Takai, Y., T. Sasaki, and T. Matozaki. 2001. Small GTP-binding proteins. Physiol. Rev. 81:153-208.

230. Takizawa, N., and Y. Murooka. 1985. Cloning of the pullulanse gene and overproduction of pullulanase in Escherichia coli and Klebsiella aerogenes. Appl. Environ. Microbiol. 49:294-298.

231. Thompson, M. R., M. J. Bjorn, P. A. Sokol, J. D. Lile, and B. H. Iglewski. 1980. Exoenzyme S: an ADP-ribosyltransferase produced by Pseudomonas aeruginosa, p. 425-433. In M. Smulson and T. Sugimara (ed.), Novel ADPribosylations of regulatory enzymes and proteins. Elsevier North-Holland Inc., Amsterdam.

232. Tonetti, D. A., C. Henning-Chubb, D. T. Yamanishi, and E. Huberman. 1994. Protein kinase $C$ - $\beta$ is required for macrophage differentiation of human HL-60 leukemia cells. J. Biol. Chem. 269:23230.

233. Towbin, H., T. Staehelin, and J. Gordon. 1979. Electrophoretic transfer of proteins from polyacrylamide gels to nitrocellulose sheets: procedure and some applications. Proc. Natl. Acad. Sci. USA 76:4350-4354.

234. Urbanowski, M. L., G. L. Lykken, and T. L. Yahr. 2005. A secreted regulatory protein couples transcription to the secretory activity of the Pseudomonas aeruginosa type III secretion system. Proc. Natl. Acad. Sci. USA 102:9930-9935. 
235. Vallis, A. J., V. Finck-Barbancon, T. L. Yahr, and D. W. Frank. 1999b. Biological effects of Pseudomonas aeruginosa type III-secreted proteins on $\mathrm{CHO}$ cells. Infect. Immun. 67:2040-2044.

236. Vallis, A. J., T. L. Yahr, J. T. Barbieri, and D. W. Frank. 1999a. Regulation of ExoS production and secretion by Pseudomonas aeruginosa in response to tissue culture conditions. Infect. Immun. 67:914-920.

237. VanAelst, L., and C. D'Souza-Schorey. 1997. Rho GTPases and signaling networks. Genes Dev. 11(18):2295-2322.

238. Vasil, M. L., D. Kabat, and B. H. Iglewski. 1977. Structure-activity relationship of an exotoxin of Pseudomonas aeruginosa. Infect. Immun. 16:353-361.

239. Vasil, M. L., and U. A. Ochsner. 1999. The response of Pseudomonas aeruginosa to iron: genetics, biochemistry and virulence. Mol. Microbiol. 34:399413.

240. Viboud, G. I., and J. B. Bliska. 2001. A bacterial type III secretion system inhibits actin polymerization to prevent pore formation in host cell membranes. EMBO J. 20:5373-5382.

241. Vincent, T. S., J. E. Fraylick, E. M. McGuffie, and J. C. Olson. 1999. ADPribosylation of oncogenic Ras proteins by Pseudomonas aeruginosa exoenzyme $S$ in vivo. Mol. Microbiol. 32:1054-1064.

242. VonPawel-Rammingen, U., M. V. Telepnev, G. Schmidt, K. Aktories, H. Wolf-Watz, and R. Rosqvist. 2000. GAP activity of the Yersinia YopE cytotoxin specifically targets the Rho pathway: a mechanism for disruption of actin microfilament structure. Mol. Microbiol. 36:737-748.

243. Weiss, A. A., F. D. Johnson, and D. L. Burns. 1993. Molecular characterization of an operon required for pertussis toxin secretion. Proc. Natl. Acad. Sci. USA 90:2970-2974.

244. Weng, B., W. C. Thompson, H.-J. Kim, R. L. Levine, and J. Moss. 1999. Modification of the ADP-ribosyltransferase and NAD glycohydrolase activities of a mammalian transferase (ADP-ribosyltransferase 5 ) by auto-ADP-ribosylation. J. Biol. Chem. 274:31797-31803. 
245. West, S. H., A. K. Sample, and L. J. Runyen-Janecky. 1994. The vfr gene product, required for Pseudomonas aeruginosa exotoxin A and protease production belongs to the cyclic AMP receptor protein family. J. Bacteriol. 176:7532-7542.

246. Whitchurch, C. B., S. A. Beatson, J. C. Comolli, T. Jakobsen, J. L. Sargent, J. J. Bertrand, J. West, M. Klausen, L. L. Waite, P. J. Kang, T. Tolker-Nielsen, J. S. Mattick, and J. N. Engel. 2005. Pseudomonas aeruginosa fimL regulates multiple virulence functions by intersecting with Vfr-modulated pathways. Mol. Microbiol. 55:1357-1378.

247. Whiteley, M., K. M. Lee, and E. P. Greenberg. 1999. Identification of genes controlled by quorum sensing in Pseudomonas aeruginosa. Proc. Natl. Acad. Sci. USA 96:13904-13909.

248. Williams, P. A., and M. J. Worsey. 1976. Ubiquity of plasmids in coding for toluene and xylene metabolism in soil bacteria: evidence for the existence of new TOL plasmids. J. Bacteriol. 125:818-828.

249. Winstanley, C., and C. A. Hart. 2001. Type III secretion systems and pathogenicity islands. J. Med. Microbiol. 50:116-126.

250. Woestyn, S., M. P. Sory, A. Boland, O. Lequenne, and G. R. Cornelis. 1996. The cytosolic SycE and SycH chaperones of Yersinia protect the region of YopE and $\mathrm{YopH}$ involved in translocation across eukaryotic cell membranes. Mol. Microbiol. 20:1261-1271.

251. Wolfgang, M. C., B. R. Kulasekara, X. Liang, D. Boyd, Q. Yang, C. G. Miyada, and S. Lory. 2003b. Conservation of genome content and virulence determinants among clinical and environmental isolates of Pseudomonas aeruginosa. Proc. Natl. Acad. Sci. USA 100:8484-8489.

252. Wolfgang, M. C., V. T. Lee, M. E. Gilmore, and S. Lory. 2003a. Coordinate regulation of bacterial virulence genes by a novel adenylate cyclase-dependent signaling pathway. Development. Cell 4:253-263.

253. Woods, D. E., J. A. Bass, J. W. G. Johanson, and D. C. Straus. 1980b. Role of adherence in the pathogenesis of Pseudomonas aeruginosa lung infection in cystic fibrosis patients. Infect. Immun. 30:694-699. 
254. Woods, D. E., D. C. Straus, J. Waldemar G. Johanson, V. K. Berry, and J. A. Bass. 1980a. Role of pili in adherence of Pseudomonas aeruginosa to mammalian buccal epithelial cells. Infect. Immun. 29:1146-1151.

255. Wurtele, M., E. Wolf, K. J. Pederson, G. Buchwald, M. R. Ahmadian, J. T. Barbieri, and A. Wittinghofer. 2001. How the Pseudomonas aeruginosa ExoS toxin downregulates rac. Nat. Struct. Biol. 8:23-26.

256. Yahr, T. L., J. T. Barbieri, and D. W. Frank. 1996a. Genetic relationship between the 53- and 49-kilodalton forms of exoenzyme S from Pseudomonas aeruginosa. J. Bacteriol. 178:1412-19.

257. Yahr, T. L., and D. W. Frank. 1994. Transcriptional organization of the transregulatory locus which controls exoenzyme S synthesis in Pseudomonas aeruginosa. J.Bacteriol. 176:3832-3838.

258. Yahr, T. L., J. Goranson, and D. W. Frank. 1996b. Exoenzyme S of Psuedomonas aeruginosa is secreted by a type III pathway. Mol. Microbiol. 22:9911003.

259. Yahr, T. L., A. K. Hovey, S. M. Kulich, and D. W. Frank. 1995. Transcriptional analysis of the Pseudomonas aeruginosa exoenzyme S structural gene. J. Bacteriol. 177:1169-1178.

260. Yahr, T. L., L. M. Mende-Mueller, M. B. Friese, and D. W. Frank. 1997. Identification of type III secreted products of the Pseudomonas aeruginosa exoenzyme S regulon. J. Bacteriol. 179:7165-7168.

261. Yahr, T. L., A. J. Vallis, M. K. Hancock, J. T. Barbieri, and D. W. Frank. 1998. ExoY, an adenylate cyclase secreted by Pseudomonas aeruginosa type III system. Proc. Natl. Acad. Sci. 95:13899-13904.

262. Yamamoto, N., N. Yamamoto, M. W. Petroll, H. D. Cavanagh, and J. V. Jester. 2005. Internalization of Pseudomonas aeruginosa is mediated by lipid rafts in contact lens-wearing rabbit and cultured human corneal epithelial cells. Invest. Ophthalmol. Vis. Sci. 46:1348-1355.

263. Yip, C. K., T. G. Kimbrough, H. B. Felise, M. Vuckovic, N. A. Thomas, R. A. Pfuetzner, E. A. Frey, B. B. Finlay, S. I. Miller, and N. C. J. Strynadka. 2005. 
Structural characterization of the molecular platform for type III secretion system assembly. Nature 435:702-707.

264. Zaas, D. W., M. J. Duncan, G. Li, J. R. Wright, and S. N. Abraham. 2005.

Pseudomonas invasion of type I pneumocytes is dependent on the expression and phosphorylation of caveolin-2. J. Biol. Chem. 280:4864-4872.

265. Zaidi, T. S., S. M. J. Fleiszig, M. J. Preston, J. B. Goldberg, and G. B. Pier. 1996. Lipopolysaccharide outer core is a ligand for corneal cell binding and ingestion of Pseudomonas aeruginosa. Invest. Ophthalmol. Vis. Sci. 37:976-986.

266. Zaidi, T. S., J. Lyczak, M. Preston, and G. B. Pier. 1999. Cystic fibrosis transmembrane conductance regulator-mediated corneal epithelial cell ingestion of Pseudomonas aeruginosa is a key component in the pathogenesis of experimental murine keratitis. Infect. Immun. 67:1481-1492.

267. Zerial, M., and H. McBride. 2001. Rab proteins as membrane organizers. Nat. Rev. Mol. Cell Biol. 2:107-119.

268. Zhang, L., H. Wang, S. C. Masters, B. Wang, J. T. Barbieri, and H. Fu. 1999. Residues of $14-3-3 \zeta$ required for activation of exoenzyme $S$ of Pseudomonas aeruginosa. Biochemistry 38:12159-12164.

269. Zupan, J. R., and P. Zambryski. 1995. Transfer of T-DNA from Agrobacterium to the plant cell. Plant Physiol. 107:1041-1047. 


\section{Curriculum Vitae}

Elizabeth Ann Rucks

Email: erucks@hsc.wvu.edu Cell phone/WV: (304)685-5899 WVU address:

PO Box 9177

Department of Microbiology, Immunology and Cell Biology West Virginia University, Morgantown, WV 26506

Morgantown, WV address:

812 Monongalia Avenue

Morgantown, WV 26505

Home: (304)296-4686 Lab: (304) 293-3951

\section{-Education:}

\section{West Virginia University, Morgantown, WV}

Doctor of Philosophy (expected, Nov. 3, 2005), January 2003-Fall 2005

Department of Microbiology, Immunology, \& Cell Biology, School of Medicine

Dissertation: Role of the host cell in the type III translocation of Pseudomonas

aeruginosa exoenzyme $S$

\section{Medical University of South Carolina, Charleston, SC}

Began training in doctoral program, August 1999-January 2003

Department of Pathology and Laboratory Medicine

Reason for leaving: My mentor, Joan C. Olson, took a position at West Virginia

University, and I was invited to relocate with the laboratory to WVU.

College of Charleston, Charleston, SC

Bachelor of Science, August 1995-May 1999

Major: Biology; Minor: Chemistry

Graduated cum laude

\section{-Publications:}

Rocha, $\mathrm{CL}^{1}$, EA Rucks ${ }^{1}$, DM Vincent ${ }^{1}$, JC Olson. 2005. Examination of the coordinate effects of Pseudomonas ExoS on Rac1. Infect. Immun. 73: 5458-5467 ${ }^{1}$ equallycontributing first authors.

Rucks, EA, JC Olson. 2005. Characterization of an ExoS type III translocation resistant cell line. Infect. Immun. 73: 638-643.

Rocha, CL, J Coburn, EA Rucks, JC Olson. 2004. Characterization of Pseudomonas aeruginosa exoenzyme $S$ as a bifunctional enzyme in J774A.1 macrophages. Infect. Immun. 71: 5296-5305. 
Rucks, EA, JE Fraylick, LM Brandt, TS Vincent, JC Olson. 2003. Cell line differences in bacterially translocated ExoS ADP-ribosyltransferase substrate specificity. Microbiology. 149: 319-331.

Fraylick, JE, EA Rucks, DM Greene, TS Vincent, JC Olson. 2002. Eukaryotic cell determination of ExoS ADP-ribosyltransferase substrate specificity. Biochem. Biophys. Res. Commun. 291: 91-100.

\section{-Awards, Honors, Committees:}

-Corporate Partner Travel Grant Award for the American Society of Microbiology $103^{\text {rd }}$ General Meeting, in Washington, DC. May 2003

-Served as student representative on the MUSC Graduate Admissions Committee, Spring 2001-December 2002

-Served as student representative on the MUSC Graduate Student Association, 2001. $-1^{\text {st }}$ place in oral presentation PhD I session of Student Research Day 2001, MUSC, Charleston, SC.

-Posters and Presentations: (*presenter)

Rucks, EA*, CL Rocha, JC Olson. Cellular targeting of Pseudomonas aeruginosa exoenzyme S to Rac1. American Society of Microbiology $104^{\text {th }}$ General Meeting, New Orleans, LA. May 2004 (Abstract and poster).

Rucks, EA* ${ }^{*}$ JC Olson. The role of the host cell in the Pseudomonas type III secretory process. Cold Spring Harbor Laboratory Microbial Pathogenesis and Host Response. CSHL, Long Island, NY. September 10-14, 2003 (Abstract and poster).

Rucks, EA* ${ }^{*}$ JT Barbieri, DM Greene, TS Vincent, JC Olson. Examining the molecular targeting of Pseudomonas aeruginosa ExoS ADP-ribosyltransferase activity within the eukaryotic cell. American Society of Microbiology $103^{\text {rd }}$ General Meeting. Washington, D.C. May 2003 (Abstract and poster).

Rocha, CL*, EA Rucks, JC Olson. Effects of Pseudomonas aeruginosa ExoS on human monocytic cell lines. American Society of Microbiology $103^{\text {rd }}$ General Meeting. Washington, D.C. May 2003 (Abstract and poster).

Greene, DM*, JM Shea*, EA Rucks, TS Vincent, JC Olson. Eukaryotic cellular processes underlying sensitivity to type III secretion of Pseudomonas aeruginosa. American Society of Microbiology $103^{\text {rd }}$ General Meeting. Washington, D.C. May 2003 (Abstract and poster).

Rucks, EA* ${ }^{*}$ JT Barbieri, TS Vincent, JC Olson. Role of the membrane localization domain of exoenzyme $S$ in determining its cellular substrate specificity. MUSC Student Research Day, 2002. Charleston, S.C. (Abstract and talk).

Greene, DM*, EA Rucks, JE Fraylick, JC Olson, TS Vincent. ADP-ribosylation and subcellular redistribution of Rac1 by exoenzyme S. MUSC Student Research Day, 2002. Charleston, S.C. (Abstract and poster).

Rucks, EA, CL Rocha, DM Greene, TS Vincent, JC Olson*. Eukaryotic cell influence on the toxic effect of Pseudomonas aeruginosa exoenzyme S. Gordon 
Research Conference: Microbial toxins and pathogenesis. Proctor Academy, Andover, New Hampshire. 2002. (Poster).

Greene, DM*, EA Rucks, JC Olson, TS Vincent. ADP-ribosylation and subcellular redistribution of Rac1 by Exoenzyme S. American Society of Microbiology $102^{\text {nd }}$ General Meeting. Salt Lake City, Utah. May, 2002. (Abstract and poster).

Rocha, CL*, EA Rucks, J Coburn, JC Olson. Exoenzyme S GAP activity contributes to the antiphagocytic capacity of $P$. aeruginosa. American Society of Microbiology $102^{\text {nd }}$ General Meeting. Salt Lake City, Utah. May, 2002. (Abstract and poster).

Rucks, EA*, JE Fraylick, DM Greene, TS Vincent, JC Olson. The role of exoenzyme $S$ in the mechanism of pathogenesis of Pseudomonas aeruginosa. American Society for Investigative Pathology Annual Meeting at Experimental Biology 2002. New Orleans, LA. April 2002. (Abstract, poster and talk).

Rucks, EA* ${ }^{\star}$, JE Fraylick, TS Vincent, JC Olson. Cell type specific ADP-ribosylation of eukaryotic substrates by Pseudomonas aeruginosa exoenzyme S. MUSC Student Research Day, 2001. Charleston, SC. (Abstract and talk-First place award).

Rocha, $\mathrm{CL}^{*}$, EA Rucks, JC Olson. Effects of P. aeruginosa exoenzyme S GAP activity on murine macrophage. MUSC Student Research Day, 2001. Charleston, SC. (Abstract and poster).

Rucks, EA, JE Fraylick, TS Vincent, JC Olson*. Differential effects of exoenzyme S on epithelial and fibroblastic cells are associated with altered ADP-ribosyltransferase substrate specificities. Gordon Research Conference: Microbial Adherence and Signal Transduction. Newport, Rhode Island. July, 2001. (Poster).

Rucks, EA* ${ }^{*}$ JE Fraylick, TS Vincent, JC Olson. Cell-type specific effects of Pseudomonas aeruginosa exoenzyme $S$ on in vivo substrate specificity and mammalian cellular function. American Society of Microbiology $101^{\text {st }}$ General Meeting. Orlando, FL. May, 2001. (Abstract and poster).

Rucks, EA* ${ }^{*}$ TS Vincent, JC Olson. The role of eukaryotic host cell in the type III mediated translocation of exoenzyme $S$ by Pseudomonas aeruginosa. American Society of Microbiology $100^{\text {th }}$ General Meeting. Los Angelos, CA. May, 2000. (Abstract and poster).

Olson, JC*, LM Brandt, EA Rucks, TS Vincent. Differential modulation of epithelial, fibroblastic and myeloid cell function by Pseudomonas aeruginosa exoenzyme $\mathrm{S}$. The $9^{\text {th }}$ European Workshop on Bacterial Protein Toxins. Ste. Maxime, France June 1999. (Abstract and poster)

Rucks, EA* ${ }^{*}$ EM McGuffie, TS Vincent, JC Olson. Factors that influence cellular sensitivity to Pseudomonas aeruginosa exoenzyme S. American Society of Microbiology $99^{\text {th }}$ General Meeting. Chicago, IL. May, 1999. (Abstract and poster).

Rucks, EA*, TS Vincent, JC Olson. Effects of Exoenzyme S of Pseudomonas aeruginosa on myelocytic HL-60 cell line. MUSC Student Research Day 1998. Charleston, SC. (Abstract and poster).

-Professional Societies:

American Society of Microbiology, member since 1998

References available upon request 
$\underline{\text { Rights and permissions }}$ 


$\begin{array}{ll}\text { From: } & \text { "Journals Rights" < Journals.Rights@oxon.blackwellpublishing.com> } \\ \text { To: } & \text { "Elizabeth Rucks" <erucks@hsc.wvu.edu> } \\ \text { Date: } & 7 / 18 / 2005 \text { 6:23:28 AM } \\ \text { Subject: } & \text { RE: Permission to reprint material }\end{array}$

Thank you for your request. Permission is granted for you to use the material you specify below subject to the usual acknowledgements (author, title of material, title of book/journal, ourselves as publisher) and on the understanding that nowhere in the original text do we acknowledge another source for the requested material. Non-exclusive World English Language, one edition, print and electronic version of publication only.

This permission is granted on the condition that you contact the author for consent should you wish to adapt/modify the material. This is not the responsibility of Blackwell Publishing.

Kind regards,

Laura Wilson.

Permissions Dept.

Blackwell Publishing

PO Box 805

9600 Garsington Road

Oxford

OX4 2ZG

United Kingdom

Fax: 00441865471150

Permission requests can now be sent to

journalsrights@oxon.blackwellpublishing.com

Blackwell is committed to creating a culture of value and respect for all of our staff. We expect to work in an environment where there are high standards of behaviour and achievement. We maintain a culture which operates within accepted boundaries of professional behaviour and performance.

----Original Message-----

From: Elizabeth Rucks [mailto:erucks@hsc.wvu.edu]

Posted At: 15 July 2005 17:46

Posted To: JULY 18th

Conversation: Permission to reprint material

Subject: Permission to reprint material

To whom it may concern:

My name is Elizabeth Rucks and I am a doctoral candidate in the Microbiology, Immunology and Cell Biology Department at West Virginia 
University in Morgantown, WV. I am writing to obtain copyright permission to use a figure for my dissertation entitled, 'Role of the host cell in the type III translocation of Pseudomonas aeruginosa exoenzyme S'.

The figure appears in a Molecular Microbiology article entitled, "Measure for measure in the control of type III secretion hook and needle length" and authored by Tohru Minamino and Anthony P. Pugsley. The full reference is Mol. Microbiol. 2005 Vol.56 Iss.2: 303-308. I would like to use Figure 1 and associated figure legend (approx. word length is 17 words) from this article.

My dissertation will be published by the Health Science Center Library, here at WVU. The distribution will be online, but will likely be password protected and have overall limited distribution. I will also have at least 8 copies of my dissertation copied and bound for distribution to my doctoral committee members, my mentor, Joan C. Olson, and myself. My dissertation is expected to be submitted for publication to the Library by the end of the Fall semester of 2005, thus appearing in limited access form by the Spring of 2006.

My contact information is as follows: Elizabeth Rucks; WVU/MICB; PO Box 9177; Morgantown, WV 26506. Phone number: 304-293-3951. Fax number: 304-293-7823.email: erucks@hsc.wvu.edu.

Thank you for your time and attention to this matter.

Sincerely,

Elizabeth A. Rucks

Elizabeth Rucks

Olson Lab

Department of Microbiology, Immunology and Cell Biology

2074 Health Science Center North

West Virginia University

Morgantown, WV 26506-9177

lab: 304-293-3951

fax $304-29.3-7823$ 


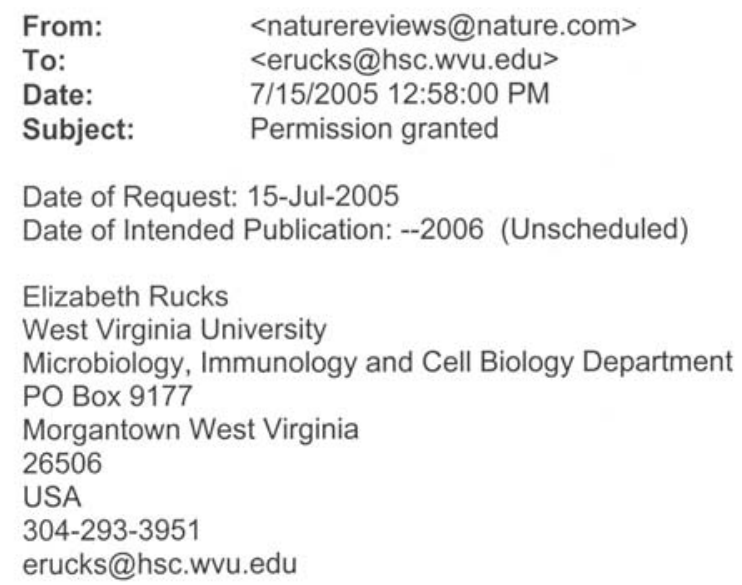

Dear Elizabeth Rucks,

Thank you for your request to use Item 1: figure, Number 1 from Nature Reviews Molecular Cell Biology, Vol. 4, pp. 738 to 743 , "Priming virulence factors for delivery into the host" by C. Erec Stebbins and Jorge Galan, et al. Print and electronic permission is granted for one-time publication in Role of the host cell in the type III trans. of Pa exoS (), by West Virginia University (Dissertation) (format: Web ), with the following specifications:

1. Reproduction is requested for a total of not more than 10 Figures/Tables in single publication, and for no other items.

2. Reproduction is intended in a primary journal, secondary journal, CD-ROM or book.

3. Nature Publishing Group's permission for electronic reproduction is not unlimited: we may in future require you to link through to the figure on Nature Online, rather than maintain an electronic version on your own web site.

4. Nature's copyright permission must be indicated next to the Figure/Table in print. In electronic form, this acknowledgement must be visible at the same time as the Figure/Table, and must be hyperlinked to the journal's homepage or at least to the Nature home page (http://www.nature.com/).

5. You must yourself obtain the consent of the author or, where such information is printed in a Nature Publishing Group journal, the photographer/illustrator.

6. Significant modifications will be faxed for approval by the authors and Nature Publishing Group.

7. Any further usage will be requested in writing.

We are certain that all parties will benefit from this agreement and wish you the best in the use of this material. Thank you.

CC:

<naturereviews@nature.com> 


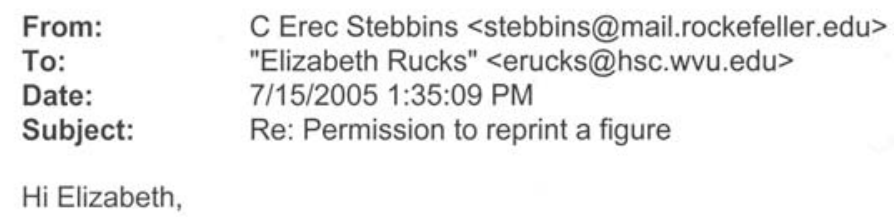

It's fine with me if you use the figure. Do you need a form signed or anything like that?

Erec Stebbins

On Jul 15, 2005, at 1:10 PM, Elizabeth Rucks wrote:

$>$ Dear Dr. Stebbins,

$>$ My name is Elizabeth Rucks and I am a doctoral candidate in the $>$ Microbiology, Immunology and Cell Biology Department at West Virginia

$>$ University in Morgantown, WV. I am writing to obtain copyright

$>$ permission to use a figure for my dissertation entitled, 'Role of the

$>$ host cell in the type III translocation of Pseudomonas aeruginosa

$>$ exoenzyme $\mathrm{S}^{\prime}$.

$>$

$>$ I would like to use Figure 1 from your's and Dr. Galan's article

$>$ entitled, 'Priming virulence factors for delivery into the host', which

> appeared in Nature Reviews Molecular Cell Biology in September 2003

$>$ (Volume 4, pages 738-743). I have received reprint permission from

$>$ Nature Reviews; however, in order to use the figure I must obtain

$>$ consent from one of the authors. If I am allowed to use the figure, the

$>$ original source will be cited, as will be your permission to use the

$>$ figure.

$>$ My dissertation will be published by the Health Science Center Library,

$>$ here at WVU. The distribution will be online, but will likely be

$>$ password protected and have overall limited distribution. I will also

$>$ have at least 8 copies of my dissertation copied and bound for

$>$ distribution to my doctoral committee members, my mentor, Joan C.

$>$ Olson,

$>$ and myself. My dissertation is expected to be submitted for publication

$>$ to the Library by the end of the Fall semester of 2005, thus appearing

$>$ in limited access form by the Spring of 2006.

> My contact information is as follows: Elizabeth Rucks; WVU/MICB; PO Box

> 9177; Morgantown, WV 26506. Phone number: 304-293-3951. Fax number:

>304-293-7823.email: erucks@hsc.wvu.edu.

$>$

$>$ Thank you for your time and attention to this matter.

$>$

$>$ Sincerely,

$>$

$>$ Elizabeth A. Rucks

$>$

$>$ Elizabeth Rucks

$>$ Olson Lab

> Department of Microbiology, Immunology and Cell Biology

$>2074$ Health Science Center North 
$>$ West Virginia University

$>$ Morgantown, WV 26506-9177

$>$ lab: 304-293-3951

$>$ fax: 304-293-7823

$>$

Erec Stebbins, PhD

Assistant Professor

Laboratory of Structural Microbiology

The Rockefeller University

email:stebbins@rockefeller.edu

Phone:(212)327-7190

Fax: (212)327-7191

http://www.rockefeller.edu/labheads/stebbins/

Mailing Address:

Laboratory of Structural Microbiology

RU Box 52

The Rockefeller University

1230 York Avenue

New York, NY 10021 
WVU/MICB

PO Box 9177

Morgantown, WV 26506

July 15,2005

ASM Press

1752 N Street, NW

Washington, DC 20036-2904

USA

Dear Ms. Ledbetter.

My name is Elizabeth Rucks and I am a doctoral candidate in the Microbiology, Immunology and Cell Biology Department at West Virginia University in Morgantown, WV. I am writing to obtain copyright permission to use a figure for my dissertation entitled, 'Role of the host cell in the type III translocation of Pseudomonas aeruginosa exoenzyme S'.

The figure appears in the book entitled Bacterial Protein Toxins, published in 2003 and edited by Drusilla L. Bums, Joseph T. Barbieri, Barbara H. Iglewski and Rino Rappuoli. The figure of interest is found on page 47 of Chapter 3: "AraC family regulators and transcriptional control of bacterial virulence determinants", authored by Dara W. Frank and Meredith L. Hunt. On page 47, Figure 3 appears as a black and white image, however many years ago, before the publication of the book, Dr. Frank gave a color copy of the figure to our lab. The color copy is also in the book on page iii of the color plates following page 256 of the book text. It is the color version of this figure that I would like to get permission to publish in my dissertation. in addition, Dr. Frank has verbally given permission for me to ask ASM press for permission to use this figure.

My dissertation will be published by the Health Science Center Library, here at WVU. The distribution will be online, but will likely be password protected and have overall limited distribution. I will also have at least 8 copies of my dissertation copied and bound for distribution to my doctoral committee members, my mentor, Joan C. Olson, and myself. My dissertation is expected to be submitted for publication to the Library by the end of the Fall semester of 2005, thus appearing in limited access form by the Spring of 2006.

My contact information is as follows: Elizabeth Rucks; WVU/MICB; PO Box 9177; Morgantown, WV 26506. Phone number: 304-293-3951. Fax number. 304-293-7823. email: erucks@hsc.wvu.edu.

Thank you for your time and attention to this matter.

Sincerely,
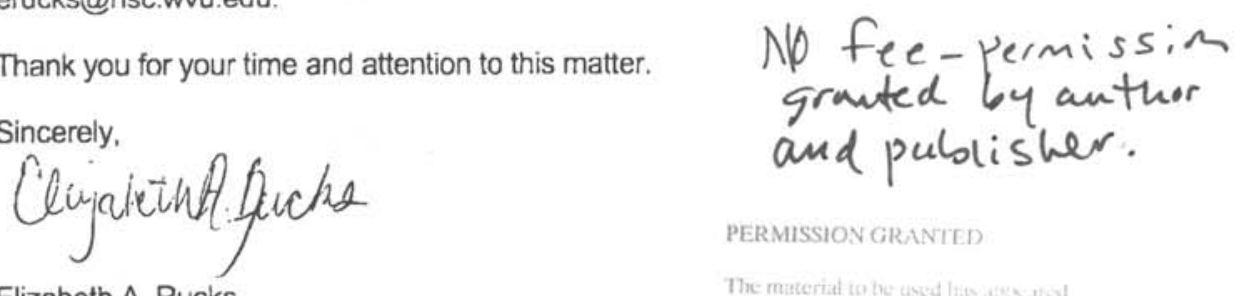

Elizabeth A. Rucks

PERMISSION GRANIED

The meteriat to be used liss any now

Doctoral candidate in the Olson lab in the Microbiology, Immunology and Cell Biology Department at West Virginia University

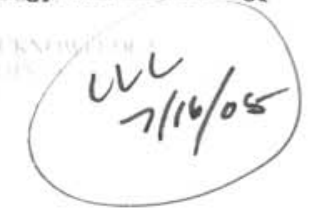


$7 / 15 / 2005$

Elizabeth Rucks

WVU/MICB

PO Box 9177

Morgantown, WV 26506

Dear Ms. Rucks:

Thank you for your request for permission to use material from Bacterial Protein Toxins by Burns et al., 2003, a publication of the ASM Press. Permission to reproduce page 256 color figure for the purpose indicated in your request is hereby granted as stated below.

1. Permission granted is on a non-exclusive, one time only basis, with distribution rights, unless otherwise stated, valid throughout the world in the English language only.

2. This permission does not allow the use of our material in any other edition or by any other means of reproduction, including (but not limited to) electronic formats, sound tapes, motion pictures, and phonograph records. This permission likewise does not include further use in book clubs, translations, abridgment, or selections which may be made of the publication. No subsequent use may be made without additional approval.

3. Appropriate credit is requested. In no specific order, please be sure to include in your reference line the ASM Press publication title, author/editor name(s), year of publication, publisher name (ASM Press), and publisher location (Washington, DC).

Sincerely,

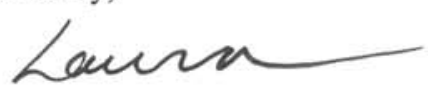

Laura Ledbetter

Editorial Assistant

Email: 1ledbetter@asmusa.org 


\begin{tabular}{|c|c|c|c|c|}
\hline $1 v V$ & & & \multicolumn{2}{|c|}{$\begin{array}{l}\text { ASM Press } \\
1752 \text { N Street, NW } \\
\text { Washington, DC 20036-2904 }\end{array}$} \\
\hline \multicolumn{3}{|l|}{ Date: $7 / 15 / 2005$} & \multicolumn{2}{|c|}{ Federal ID \#: 38-1616141 } \\
\hline \multicolumn{5}{|l|}{ Invoice \#: 2005.61} \\
\hline \multicolumn{5}{|c|}{$\begin{array}{l}\text { Bill To: Elizabeth Rucks } \\
\text { WVU/MICB } \\
\text { PO Box } 9177 \\
\text { Morgantown, WV } 26506\end{array}$} \\
\hline CONTACT & Dept & Fax \# & \multicolumn{2}{|c|}{ TERMS } \\
\hline Laura Ledbetter & ASM Press & 202.942 .9342 & \multicolumn{2}{|c|}{90 days } \\
\hline $\begin{array}{c}\text { \# of Figures or } \\
\text { Pages }\end{array}$ & $\begin{array}{c}\text { \# of Copies } \\
\text { per page (optional) }\end{array}$ & DESCRIPTION & UNIT PRICE & TOTAL \\
\hline 1 figure & 1 figure & $\begin{array}{l}\text { Permission to use page } 256 \text { color figure from our } \\
\text { book Bacterial Protein Toxins by Burns et al., } \\
2003 \text { for use in your dissertation }\end{array}$ & No Fee & No Fee \\
\hline & & & TOTAL DUE & No Fee \\
\hline
\end{tabular}

Please make check payable to the ASM Press. All payments from outside the U.S. must be in U.S. dollars, drawn on a bank located within the continental United States. A copv of this invoice must be included with payment before signed approval for permission will be returned. Return payment to the attention of Laura Ledbetter, ASM Press (mailing address listed above) as soon as possible. If you wish to make payment by way of electronic transfer, you will need to contact me for bank information. Payments under US $\$ 50$ not accepted by electronic transfer.

For questions or additional information, please contact ASM Press.

\section{THANK YOU!}

Laura Ledbetter

Editorial Assistant

ASM Press

Tel: 202.942 .9203

Fax: 202.942.9342

Email: 1ledbetter@asmusa.org 


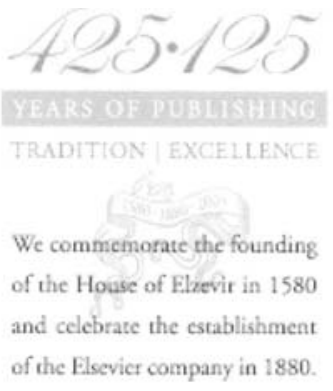

Our Ref: HG/smc/August 2005.199

Ms Elizabeth A Rucks

erucks@hsc.wvu.edu

Dear Ms Rucks

BIOCHEMICAL AND BIOPHYSICAL RESEARCH COMMUNICATIONS, Vol 291, No 1, 2002, Pages 91 100, Fraylick et al, 'Eukaryotic Cell ...', 2 Figures only

Proposed Use: 'Eukaryotic cell determination of ExoS ADP-ribosyltransferase substrate specificity'

As per your letter dated 22 July 2005, we hereby grant you permission to reproduce the aforementioned material in print and electronic format at no charge subject to the following conditions:

1. If any part of the material to be used (for example, figures) has appeared in our publication with credit or acknowledgement to another source, permission must also be sought from that source. If such permission is not obtained then that material may not be included in your publication/copies.

2. Suitable acknowledgement to the source must be made, either as a footnote or in a reference list at the end of your publication, as follows:

"Reprinted from Publication title, Vol number, Author(s), Title of article, Pages No., Copyright (Year), with permission from Elsevier".

3. This permission is granted for non-exclusive world rights in all languages.

4. Reproduction of this material is granted for the purpose for which permission is hereby given, and includes use in any future editions.

Yours sincerely
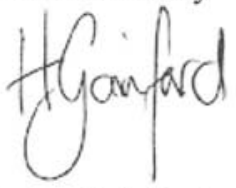

Helen Gainford

Rights Manager

Your future requests will be handled more quickly if you complete the online form at www.elsevier.com/locate/permissions 


\title{
micr $\oplus$ biology
}

Published by the Society for General Microbiology

\author{
Elizabeth Rucks \\ Olson Lab \\ Department of Microbiology, Immunology and Cell Biology \\ 2074 Health Science Center North \\ West Virginia University \\ Morgantown, WV 26506-9177, USA
}

19 May 2005

\section{Dear Ms Rucks}

On behalf of the Society for General Microbiology as copyright holder I am happy to grant permission for this material (paper no. 25985) to be reproduced, provided that the source is acknowledged.

Yours sincerely

Kendra Waite

Microbiology Editorial Assistant

\footnotetext{
Editorial Office: Society for General Microbinlngy, Marlborough House, Basingstoke Road, Spencers Wood, Reading RG7 1AG, UK $T+44(0) 1189881804$ $F+44(0) 1189881634$ E micro@sgmac.uk $W$ mic.sgmjournals.ora

Reaistered Charity No. 26401 ? Combanv Limited bx Guaranier Rroisterert in Enaland No. 1033582 Reaistered Office as above
} 


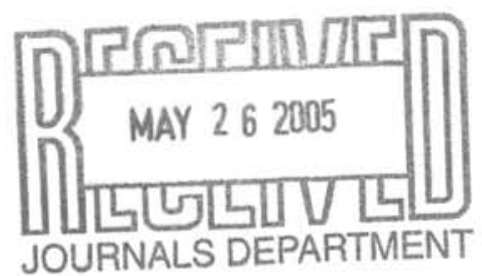

May 18, 2005

Journals Department

American Society for Microbiology

1752 N Street, N. W.

Washington, D.C. 20036-2904

\section{Dear Sir or Madam:}

I am writing to ask permission to reprint a paper, of which I am first author, as a chapter of my Dissertation. The paper entitled, 'Characterization of an ExoS Type III Translocation-Resistant Cell Line' was published in the January 2005 (volume 73, issue 1, pages 638-643) issue of Infection and Immunity. This work is a significant part of my doctoral thesis, and therefore, I would like to use figures and text that were originally published in this article.

Copyright permission can be remitted to Elizabeth Rucks; Department of Microbiology, Immunology and Cell Biology; West Virginia University; PO Box 9177; Morgantown, WV 26506 or to fax number 304-293-7823. Thank you for your time and consideration.

Sincerely,

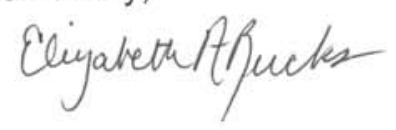

Elizabeth A. Rucks

Graduate student in Joan C. Olson's Laboratory

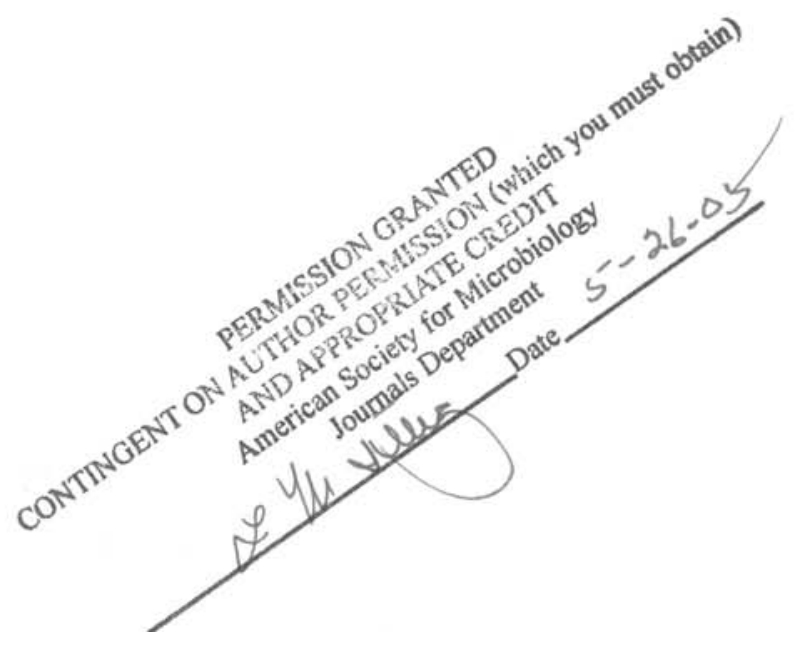

